\title{
Abstracts of the 66th Annual Conference of IACTS, February 2020
}

\author{
CONCOMITANT PULMONARY \\ VALVE REPLACEMNT WITH \\ INTRACARDIAC REPAIR FOR \\ ADULT TOF \\ Dr. Kartik Patel, Dr. Anil Jain, \\ Dr. Suresh kumar Rajan, Dr. Pankaj Garg, \\ Dr. Vishal Agrawal, Dr. Deepti Kakkar, \\ Dr. Trushar Gajjar, Dr. Amit Mishra, \\ Mr. Sanjay Patel, Dr. Chirag Doshi. \\ U.N. Mehta Institute of Cardiology \\ and Research Center
}

\section{Objectives:}

There is still a limited data available about the effect of concomitant Pulmonary Valve Replacement (PVR) with Tetralogy of Fallot (TOF) repair on the heart and exercise capacity in adolescent and adults. The aim of our retrospective study was to examine the effect of concomitant PVRon RV parameters, cardiopulmonary exercise capacity and bioprosthetic valve durability at mid-term.

Method and Materials:

Between January-2013 and August-2018, 37 adolescents and adults with TOF who had hypoplastic pulmonary annulus underwent concomitant TOF repair with PVR at our institute. We retrospectively collected the data from the hospital records including follow up.

Results:

Mean age of the patients was $18.48 \hat{\mathrm{A}} \pm 7.53$ years. Bioprosthetic valve size ranged from $19 \mathrm{~mm}$ to $25 \mathrm{~mm}$. There was no early or late mortality. No patient had developed low cardiac output syndrome (LCOS), acute renal failure, ventricular dysfunction, arrhythmias, or sepsis. Follow-up was complete and at a mean duration of $41.3 \pm 10.4$ months, no patient required reoperation or re-interventions. Mean QRS duration, RV function, $\mathrm{RV}$ end systolic and end diastolic dimensions, Prosthetic valve gradient, RV myocardial performance index and Functional status(including NYHA class and 6-minute walk test) were not significantly different as compared to pre-discharge value. During follow-up, 2 patients $(5.4 \%)$ developed moderate PR, and 2 patients (5.4\%) developed moderate PS. Conclusion:

Concomitant PVR with TOF repair in adult provides excellent early and midterm outcome, with acceptable rate of pulmonary valve degeneration at midterm. It not only smoothens the early postoperative course but, also preserves the right ventricular function as well as functional status at mid-term.

\section{EXPERIENCE WITH USE OF SINGLE INTERNAL MAMMARY ARTERY VS BILATERAL INTERNAL MAMMARY ARTERY IN CORONARY BYPASS GRAFTING}

Prof. Dr. James Thomas, Dr. Abhay Jain, Dr. Mahesh Singh, Dr. Umbarkar, Dr. Abiah Jacob D.Y. Patil University Hospital, Administration 3rd Floor, Sector 5, Nerul, Navi Mumbai 400706.

\begin{abstract}
Aim:
Coronary Artery Bypass Grafting is highly effective for relief of symptoms in multi vessels and left main coronary artery disease. Superior patency of LIMA compared to saphenous vein is conclusive. Left IMA is established as standard of care for grafting the Left Anterior Descending Artery LAD. The aim of presentation to compare early outcomes of patients receiving single IMA vs Bilateral IMA.

Methods:

Between 2008 and 20191422 patients underwent CABG using Single IMA (personal series) in addition to vein grafts and radial artery grafts in few cases. Bilateral IMA was performed in 840 patients (Drs. MS and $\mathrm{RU})$ during the same period. Youngest patients to receive single IMA was 34 year female and 35 year male and the oldest was 77 and 88. Male female ratio was $60: 40$ and $45 \%$ of patients below 60 years. Incidence of diabetes in both groups were $70 \%$ one of the CABG in single IMA group had mobile thrombus in the left ventrical and 4 patients had post infarction VSD repaired in addition to CABG.18\% of the all anastomosis was performed on cardio pulmonary bypass with cardio pledgic arrest using both antigrade and retrograde. Rest were on OPCAB, off pump bypass. Skeletonization of RIMA was done in most cases of BIMA to achieve higher anastomosis in circumflex OM and RPDA. RIMA was anastomosis directly to RCA in some cases. In single IMA group vein graft were used sequentially also.

Results:

Mortality in SIMA vs BIMA were 7\% and $8 \%$ incidence of perioperative MI, Renal failure, reoperation for bleeding, strokes did not differ. Slight higher incidence of mediastinitis was $3.2 \%$ vs $2 \%$ in single IMA. Angiography and CT Angiography were done in 24 patients 6 months to 10 years showed graft patency $95 \%$ IMA and $84 \%$ venous graft.

Conclusion:

Low use of Bilateral IMA is still prevalent in many centres. Increased technical complexity, incidence of diabetes milletus, dIffuse disease, potential increase in mortality and mobility has prompted many to continue with single IMA with venous grafts with additional radial artery grafts. 85 to $90 \%$ of bypass surgery performed all over the world could be Single IMA with vein grafts. With experience using both IMAs can be performed with good clinical results and low morbility.
\end{abstract}

\section{EXTRA GONADAL INTRATHORACIC NON SEMINOMATOUS GERM CELL TUMOUR}

Bharath Bhogavalli, Amaresh

Rao Mallempati, PSS Gopal

Nizams Institute of Medical Sciences

We present a case of 13-year-old male, presented with c/o weakness of right upper and lower limbs, urinary incontinence, and back pain, on evaluation found to have epidural metastasis and mediastinal mass. $\mathrm{He}$ is a known case of NON SEMINOMATOUS GERM CELL TUMOR evaluated at a private hospital. Took 10 cycles of radiotherapy for epiduralmetastasis and 4 cycles of chemotherapy. The chest radiograph 
revealed complete opacification of left chest which was confirmed by computed tomography as a large left mediastinal mass measuring 15 $\tilde{\mathrm{A}}-10 \tilde{\mathrm{A}}-8 \mathrm{a} € \%$ cm. Classic median sternotomy was done and tumor debulked in Toto. Histology confirmed non semi-nomatous germ cell tumor component. We describe this rare and large thoracic tumor and discuss how surgical management helps treatment of this exceptional tumor.

\section{LEFT SINUS OF VALSALVA ANEURYSM RUPTURING IN TO SUPERIOR VENACAVA \\ Dr VPL Chandrakumar Sistla, \\ Dr Ajit Kumar Padhy, Dr Anubhav Gupta \\ Vardhman Mahavir Medical college and \\ Safdarjung Hospital, New Delhi}

Sinus of Valsalva aneurysms arising from the left coronary cusp, and rupturing into superior venacava is a rare occurrence. To the best of our knowledge, this aberration has not been reported yet. Advancement in recent diagnostic techniques make the planning of treatment easier. The complexity of its anatomical course averts any catheter-based intervention where in surgical closure remains the ultimate choice of treatment. We performed a bicameral approach for a23-year old female patient who was diagnosed with â€œ Sinus of Valsalva aneurysmarising from the left coronary sinus opening in to superior Venacavaâ€. Postoperative period and follow up were uneventful with no residual fistula.

\section{COMPLETE STERNAL CLEFT WITH CONGENITAL HEART DEFECT: REPORT OF TWO CASES OF COMBINED REPAIR} Pratik Pandey, Debasis Das, Nilanjan Dutta,

Shubhodip Das, Manish Sharma, Rangan

Koley, Amitabh Chatterjee

Department of cardiac surgery, cardiac

anesthesia, pediatric cardiology- Narayana

Superspeciality Hospital Howrah Kolkata

\section{Background:}

Cleft sternum is extremely rare congenital midline defect of the sternum. It is not uncommonly associated with intra cardiac defects. We report 2 cases of successful combined repair ( cardiac procedure +sternal wall reconstruction). The first case is a 2 year old child with complete sternal cleft and tetralogy of Fallot who presented with cyanotic spells. The child underwent total correction followed by chest wall reconstruction on the next day. The other patient is also 2 year old with Tricuspid Atresia, Pulmonary stenosis (Univentricular Heart). This child underwent a Glenn Shunt and sternal wall reconstruction in same sitting. We used bilateral oblique sliding chondrotomies of first four ribs followed by mobilization of bony and muscle cover as the sternal reconstruction.

Aims/ Objectives;

To report two cases of Total sternal cleft with congenital cardiac defect, Avery rare association and technique of creation of neosternum to provide effective bony cover over heart.

\section{Material and Method:}

We report a technique whereby Bilateral sliding chondrotomy with pectoralis major muscle advancement is done to repair congenital absent sternum in a noncompliant chest wall.

Results:

We have used this technique in two cases of absent sternum with congenital cardiac anomaly and on follow-up chest wall is compliant with no paradoxical movement and there is strong bony cover protecting heart.

\section{Conclusions:}

Absent cleft sternum with associated congenital cardiac defect is a very rare entity. Re-establishment of bony cover over mediastinal structures in a noncompliant chest wall is a challenge. Our surgical technique is simple and can be performed in same sitting ( as aconcomitant procedure along with cardiac surgery)

\section{IATROGENIC ARTERIOVENOUS \\ FISTULA INVOLVING THE \\ RADIAL ARTERY AND CEPHALIC VEIN OF RIGHT UPPER LIMB: MANIFESTING AS ANEURYSMS OF THE SAME \\ Hemant Bodhankar, Suraj Nagre,Saptrishi paul Grant Medical College, Mumbai, Maharashtra}

Arteriovenous Fistula (AVF) is defined as an anomalous communication between an artery and avein. Iatrogenic AVFs are a rare clinical finding compared to the congenital type. They are most commonly secondary to penetrating and blunt trauma, iatrogenic complications of invasive procedures and operations. Although still rare iatrogenic AVFs are more commonly reported now due to the increased use of percutaneous diagnostic and interventional endovascular procedures. Here we present the case report of a 22 year old female, who had undergone intravenouscannulation in the right forearm volar aspect, for intravenous antibiotics, asa treatment of typhoid fever. One week after the procedure, she developed an small aneurysmal swelling in the volar aspect of the right forearm which increased to size of 4 by $5 \mathrm{~cm}$ over six months. On computed tomography revealed two aneurysms lying next to each other with acommunication akin to a fistula. The swellings were surgically resected completely with preserved vascularity.

\section{RARE SOLITARY PULMONARY METASTASIS AFTER 1OYEARS OF TREATED PRIMARY CERVICAL MALIGNANCY}

Senthil Vaithiyanathan, Sivaraman Amaravathi Institute of Cardiothoracic surgery, Madras medical college

\section{Background:}

Pulmonary metastases were detected in $83.9 \%$ patient within 2 years after the initial treatment of cervical cancer, and disease free interval between the primary cervical malignancy and secondary lung metastasis was within 2 to 5 years, the median survival after lung metastasis was 18 months with 2 and 5 year survival rate of $37.7 \%$ and $7.5 \%$ respectively, patients with cervical cancer $2.1 \%$ to $6.1 \%$ patients develop lung metastasis but a secondary solitary lung metastasis after 10 years of a treated primary cervical malignancy is rare.

\section{Methods:}

47, female with $\mathrm{h} / \mathrm{o}$ total abdominal hysterectomy for carcinoma cervix 10years back not on follow up present with hemoptysis and left chest pain. Now CECT chest shows $2.5 \times 2 \mathrm{~cm}$ well defined hyper dense nodule noted in the posterior basal segments of left lower lobe with significant contrast enhancement. CT guided biopsy of the mass reveals metastatic squamous cell carcinomatous deposit. She underwent left lower lobectomy and post of HPE confirm diagnosis

Conclusion: Secondary pulmonary metastasis after 5years of treated primary cervical malignancy is rare we report a case of solitary secondary lung metastasis after 10years of treated primary cervical malignancy 
A RETROSPECTIVE ANALYSIS OF AORTIC VALVE REPAIR WITH SURGICAL CLOSURE OF VENTRICULAR SEPTAL DEFECT

DR. Aryakamal Das, DR. Debasis

Das, DR. Nilanjan Dutta,

DR. Shubhadeep Das, DR. Amitava

Chattopadhyay, DR. Rangan

Koley, DR. Manish Sharma

Narayana Super Speciality

Hospital, Howrah, West Bengal

\section{Objective:}

To analyse the short term outcome of Aortic Valve repair associated with VSD Closure.

\section{Methods:}

A retrospective analysis of the patients from 1 to 20 years of age operated from January 2016 to April 2019in a single tertiary care level institution. Patients were included if they required any type of Aortic Valve repair during VSD closure for significant aortic valve regurgitation. All data we regathered from patients medical records and operative reports. Follow up data was also collected.

\section{Results:}

16 patients were included in this study. Aortic Valve repair along with VSD closure was done for all patients with more than moderate AR detected on pre-operative .The severity of AR was moderate (6), moderate to severe(3) and severe(7). 2 patients had left ventricular dysfunction with EF-40\%. The types of Aortic Valve repair done were Modified Trussler's with Sub Commisural Annuloplasty in 7 cases, Trussler's repair in 8 cases and Trussler's repair with Sub-Commisural Annuloplasty in 1 case. AR at discharges were No AR (1), Mild (14) and Moderate (1). During followup of average 6 months there were no further increase in AR compared to till now. Conclusion:

Aortic valve repair is an effective technique for dealing with significant AR during the time of VSD closure. A combination of various techniques is usually needed. In our experience AV repair should be a preferred strategy in children with VSD with AR with a detailed pre-op TEE to delineate the cause of $\mathrm{AR}$ and dimensions and strict follow-up.

\section{RUPTURED SINUS OF VALSALVA ANEURYSM FROM LEFT CORONARY SINUS TO RIGHT ATRIUM - A RARE CASE PRESENTATION \\ DR. Madasu Sudhakara Rao, \\ DR. Anand Agarwalm, DR. S. Vengal Reddy \\ Malla Reddy Narayana \\ Multispeciality Hospital, \\ Jeedimetla, Hyderabad}

A 33 years old female presented with chest pain on exertion and progressive shortness of breath for the past 10 days. On examination she had a loud continuous murmur, elevated JVP and dry cough. The examination findings, electrocardiogram, chest radiography and echo cardiogram are discussed in a step-wise manner to arrive at a diagnosis and plan of management of a patient with RSOV is discussed along with surgical video presentation. The case is being presented for its rarity. Key words: continuous murmur, RSOV, Left Coronary Cusp sinus

\section{MITRAL STENOSIS WITH SADDLE EMBOLUS- REPORT OF TWO CASES OF MITRAL VALVE REPLACEMENT WITH EMBOLECTOMY IN SAME SITTING}

Pratik Pandey, Lalit Kapoor, Dhiraj Barman

Rabindranath Tagore International Institute of Cardiac Sciences Kolkata
Background:

Mitral stenosis is a frequently associated with a trial fibrillation and left atrial clots and resultant peripheral embolization with outcomes ranging from mild TIAs to severe hemiplegia, limb ischaemia and even death. A large saddle embolus in presence of mitral valve disease has dreaded tendency to recur in a few weeks and often even in the same hospital admission.

\section{Material \& Methods:}

We report a rare experience with two such patients having aortic saddle with mitral stenosis. Bilateral femoralembolectomy was carried out in both cases with exposure of both femorals after heparinization. Once adequate clot removal was achieved, flow re-established, and the femorals were repaired, mitral valve replacement (MVR) was carried out routinely at the same sitting. Our reasoning was that the patients were eligible candidates for MVR.

\section{Results:}

Both patients recovered from the surgeriesuneventfully without any problems with limb salvage. One patient developed peronealnerve palsy which resolved in two months with conservative treatment.

Conclusion:

Embolectomy of large saddle embolus can be safely carried out with planned MVR in the same sitting without significant complication. This also reduces the chance of re-embolization in the interim period.

\section{CROSSING THE BRIDGES TO \\ TRANSPLANTATION}

Jeevesh John Thomas, Bhaskar

Ranganathan, Chengalath Manoras Mathew,

Simon Philipose, Shylesh Kumar, Jose

Chacko Periapuram

Lisie Heart Institute, Lisie Hospital,

Kaloor, Kochi, Kerala

\section{Background:}

Cardiac transplantation still remains the gold standard for end stage heart failure. Getting the patient to a transplant still remains a challenge. The long waiting list for transplant, the presence of other organ dysfunctions, raised pulmonary pressures etc. are some of the challenges faced by the transplant team. The patient in this waiting period may require inotropic support or sometimes mechanical circulatory support systems like balloon pumps, Extra Corporeal Membrane Oxygenaration (ECMO), or Ventricular Assist Devices (VAD).

Method and results:

We describe a patient who had to be supported on 2 different mechanical support devices before transplantation. A 32 year old software engineer was detected to have end stage cardiac failure from ischemic Cardiomyopathyand was referred to us for a possible cardiac transplantation. He had secondary renal and hepatic dysfunctions and was on Dobutamine infusion. He had a sudden cardiac arrest while on workup and an emergency ECMO had to be instituted. Central ECMO with aortic canula and both left and right atrial venous canulas was established and sternum was left open. Since the right ventricle was still strained, decided to support on Biventricular Assist Device (BiVAD) and hence Centrimag BiVAD was established after 3 days on ECMO. Sternum was closed and patient was extubated after 2 days. He was maintained on BiVAD for 14 days. An orthotopicheart transplant was done on 10/10/16. Ischemic time was 4 hours including a travel time of 1.5 hours. Post operative recovery was smooth with IABP support for 48 hours. He is now 3 years post op and is doing well. 1 episode of grade 2 rejection was treated with pulse steroids. His coronary angio done at 1.5 years was normal.

Conclusion:

ECMO and VAD can be used as successful bridges to eventual transplant if used diligently. LVAD can be used as a destination therapy in future if waiting lists become too long. However as of now heart transplant isstill gold standard. 


\section{TRACHEA STENOSIS}

Prof. Rajan Santosham,

Dr. Rajiv Santosham, Dr. David Idowu

Santosham Chest Hospital

\section{Background:}

Trachea stenosis, a challenging problem, is an established complication of prolonged intubation and tracheostomy. It's a therapeutic challenge that requires a multidisciplinary approach.

\section{Case Presentation:}

This is a video presentation of a 32-year-old man who had recurrent tracheastenosis following prolonged intubation and tracheostomy and subsequent tracheasurgery and other interventional procedures. He had trachea resection and end-to-end anastomosis was done with a good outcome.

Take-Home Message:

Trachea surgery requires careful surgical management. Optimum preoperative planning requires radiological (computerized tomography scan) and bronchoscopic evaluation. Use of laryngeal mask airway for intubationallow intraoperative delineation of the stenotic segment. Marking of the site of trachea incision with a needle. Avoiding injury to the recurrent laryngeal nerve. Good trachea mobilization. Tension-free anastomosis. Interrupted vicryl sutures. Ensure air-tight anastomosis (No air-leak). Grilloa's stitch to avoid hyperextension of the neck.

\section{PAPILLARY FIBROELASTOMA OF AORTIC VALVE}

Dr. Uma Karthik, Dr. Amaresh Rao

Nizams Institute of Medical

Sciences

We present a case of 25-year-old female, presented with c/o chest pain , on evaluation found to have pedunculated mass arising from RCC OF AORTIC VALVE. 2D Echocardiography and TEE showed mobile pedunculated mass $(0.9 \tilde{\mathrm{A}}-0.9 \mathrm{cms})$ arising from RCC of aortic valve. Other investigations are within limits. She was operated with excision of pedunculated mass on cardio pulmonary bypass. Histology of mass came as PAPILLARY FIBROELASTOMA. We describe this rare primary cardiac tumour case and discuss how surgical management helps treatment of this exceptional case

\section{ANEURYSM OR PSEUDO \\ ANEURYSM- WHAT'S IN A NAME? \\ Jeevesh John Thomas, Bhaskar Ranganathan, \\ Simon Philipose, Shylesh Kumar, \\ Jose Chacko Periapuram \\ Lisie Heart Institute, Lisie Hospital, Kochi, Kerala}

\section{Introduction:}

True left ventricular aneurysms are widely recognized as a common and serious complication following total occlusion of coronary arteries with a prevalence of approximately $12 \%$ of acute transmural myocardial infarctions. Accordingto the available scientific literature, the apex and the posterior wall are the most common sites of aneurysm formation (31.7\% and $23.8 \%$, respectively). Theanterior wall and the intraventricular septum are less frequently involved $(9.5 \%$ and $7.9 \%$, respectively). As few as $1 \%$ of ventricular aneurysms affect the lateral wall of the ventricle and are usually not extensive. Rupture of the free wall of the left ventricle (LV) is a catastrophic complication occurring in $4 \%$ of patients after myocardial infarction (MI) and in $23 \%$ of those who die of MI. Rarely the rupture is contained by an adherent pericardium creating a pseudo-aneurysm. Differentiation between these two pathologies remains difficult. We here in describe a case of lateral wall aneurysm which was initially diagnosed as a pseudoaneurysm on imaging studies.

\section{Method and result:}

A 71 year old man was referred to us with a diagnosis of LV aneurysm/ pseudo aneurysm and ischemic ventricular tachycardia. He had a CABG done in 2003 with LIMA to LAD and diagonal. Re angiogram showed patent LIMA graft. Circumflex was occluded and right coronary (RCA) had multiple flow limiting lesions. MRI was suggestive of large wide mouthed aneurysm from lateral and posterolateral wall of LV with only pericardium seen in some areas-? Pseudo aneurysm. Per operatively, a large thin walled aneurysm involving the circumflex territory was seen with thrombus within. The base of the aneurysm was abutting the papillary muscles. Under cardioplegic arrest, the aneurysm was opened and clots removed. Using pledgetted 3-0 prolene sutures, a PTFE patch was sewn over the neck of the aneurysm. The aneurysm was then wrapped around the patch using 2-0 prolene with felt strips on either side. Vein grafts were connected to the posterior descending and posterolateral branches of RCA. The patient was weaned off bypass with balloon pump support; the chest was left open for a day and closed after 24 hours. He had thereafter an uneventful recovery. Conclusion:

Left ventricular aneurysm involving the lateral and posterior wall is rare and surgery is difficult especially if the mitral apparatus is involved. A timely diagnosis and early surgical treatment is vital in patient management to prevent fatal cardiac rupture.

\section{VASOPLEGIA VERSUS SEPTIC SHOCK AFTER CARDIAC SURGERY- DIFFERENTIATION AND MANAGEMENT}

Dr. Rakesh Chittora, Dr. Vinita Sharma,

Dr. Mukta Bhatnagar

Fortis Escorts Hospital, Jaipur

Vasoplegia syndrome is critical condition in past cardiac surgery patient which in characterized by generalized hypotension related to very law systemic vascular resistance refractory to routine vasopressor agents. Cardio-pulmonary bypass is a strong precipitant of the Vasoplegia syndrome, largely due to its association with nitric oxide production and severe vasopressin deficiency. Various risk factors like initial patient condition, cardiac procedure type, pre-operative medication, use of cardio pulmonary bypass, intrinsic factors leading to vasodilatation or infectious factors should be taken into consideration while dealing with this condition. Vasoplegia syndrome mimics septic shock and therefore itâ€ $€^{\mathrm{TM}_{S}}$ creates dilemma and great challenge while treating this condition in terms of confusion in deciding between escalation of vasopressor agent and escalation of antibiotics. Key Words: Vasoplegia, Septic shock, CPB antibiotic prophylaxis

\section{CHRONIC THROMBOEMBOLIC PULMONARY HYPERTENSION MANAGEMENT; OUR EXPERIENCE WITH SHORT TERM FOLLOW UP \\ Kuntal Surana, J. V. Khandekar, \\ Prashant Mishra, Chaitanya \\ Raut, Vaibhav Shah, Manish Jadhao \\ Lokmanya Tilak Municipal \\ Medical College and General Hospital}

\section{Objectives:}

Pulmonaryendarterectomy (PEA) is the only curative treatment for patients suffering from chronic thromboembolic pulmonary hypertension $(\mathrm{CTEPH})$. We reviewed patient characteristics, outcome and factors affecting outcome. 


\section{Methods:}

Between 2014 and 2019, 45 patients underwent PEA for CTEPH at our institution. All patient data were entered in a prospective database. We performed a retrospective analysis of our total patient collective, to assess patient characteristics, outcome after PEA and factors affecting outcome after surgery.

Results:

There were 4in-hospital deaths. Nonsurvivors had significantly higher mean pulmonary arterial pressure and pulmonary vascular resistance than did survivors. There was a significant reduction in mean pulmonary artery pressure (mPAP) (from 51 to $33 \mathrm{mmHg}$ ) and pulmonary vascular resistence (PVR) (860 to 337 dynes). At 1-year follow-up, 90\% of patients were alive and hemodynamic improvements were sustained in the majority of patients. Age, mean pulmonary artery pressure higher than $55 \mathrm{~mm}$ hg, a PVR of higher than 850 dynes.s.cm-5 and NYHA functional class IV were predictors of in-hospital and 1 year follow up mortality. Conclusion:

In our experience PEA provided immediate and substantial improvements in pulmonary hemodynamics and had sustained favorable effects on 1 year follow up. High preoperative PVR was a significant independent risk factor for in-hospital death. More studies including a larger cohort of patients preferably performed in multiple centers are needed to assess long term results and find out other factors affecting outcome.

\section{CONE REPAIR- OUR 5 YEAR EXPERIENCE}

Sujoy Chatterjee, Rajarshi Ghosh, Santosh

Pandey, Lakshmi Sankhayan, Nikhil Bansal,

Srirup Chatterjee, Satyajit Bose

The Mission Hospital, Durgapur

\section{Introduction:}

The Cone Repair of Tricuspid Valve in patients with Ebstein's anomaly was first described by da Silva and subsequently modified by Dearani and others has become the repair of choice for these patients.

Materials and methods:

From January 2014 to May 2019, total 43 patients ( Median age 10.3 years, range 4.1 to 16 years ) underwent Cone Repair at our institute. All patients had plication of atrialised RV. 3 patients has additional Bidirectional Glenn shunt because of inadequate functional RV.

\section{Results:}

Median follow up was 23 months ( 7 to 60 months). There was one early death due to progressive RV failure within 30 days of surgery. All patients were followed up every 6 months by echo cardiograpgy. 30 patients had nil or mild TR, 11 patients had moderate TR, 1 patient was lost to follow up. All patients had adequate RV function. None of our patients required Tricuspid Valve Replacement during the study period.

Conclusion:

Cone repair is the best treatment option for patients with Ebsteins anomaly across a wide range of age group, Further follow up is of these patients is required to ascertain the quality of life, functional capacity and freedom from reintervention.

1000 PATIENTS OF CLOSED MITRAL COMMISSUROTOMY 25 YEARS EXPERIENCE FROM GOVT RAJAJI HOSPITAL MADURAI MEDICAL COLLEGE, TAMIL NADU

Dr. A.S. Mughilan, Rani P MS MCh,, Karthikeyan B MS MCh, Marvin Manoah

Baylis MS MCh, Department of

Cardiovascular Thoracic Surgery, Madurai Medical College

Madurai Medical College,

Madurai, Tamil Nadu
Aim / objectives:

Valve preserving surgery is superior than valve replacement. Valve replacement in a rural area patient - very difficult to follow up. Dosage of anticoagulant and its effects varies in different individuals. Monitoring all patients with INR all the times in a patient from remote area is very difficult. So, preserving native valve by doing CMC prolongs patient's survival with good quality of life.

Materials/ methods:

Patients who have undergone CMC for RHD with MS with or without LAA clot for the past 25 years from our institute.

\section{Results:}

In our study, patients RHD MS with moderately calcified leaflets and commissural calcification with / without LAA clot who undergone CMC \&gt; $90 \%$ patients leads good quality of life with medical management without anticoagulants, comparing with mitral valve replacement.

\section{Conclusions:}

Closed mitral commissurotomy for rheumatic mitral valve stenosis was used in many tens of thousands of patients with excellent immediate results and durability, many patients having survived more than 20 years without re-operations and without anticoagulants. Though this procedure was displaced by the open commissurotomy, mitral valve repair with an immediate assessment of the anatomical and functional result. But because of its deleterious effects of cardiopulmonary bypass, closed mitral commissurotomyis still considered a better line of management for a selected group of patients.

\section{HIGH FLOW NASAL CANNULA THERAPY VERSUS CONVENTIONAL OXYGEN THERAPY FOR PEDIATRIC PATIENTS UNDERGOING CARDIAC SURGERY POST-EXTUBATION : A RANDOMIZED CONTROLLED TRIAL. \\ Dr. Noaman K Shaikh, Dr. Arnab De, \\ Dr. Tejas Dandekar, Dr. Vaibhav Shah, \\ Dr. Manish Jadhao, Dr. Chaitanya Raut, \\ Dr. Prashant Mishra, Dr. Jayant V Khandekar \\ Lokmanya Tilak Municipal Medical College and Hospital}

\section{Introduction:}

High-flow nasal cannula (HFNC) use has greatly increased in recent years. High-flow gas is defined as a flow that meets or exceeds the patient's inspiratory flow (reference). Without heated humidity, the amount of gas flow delivered via nasal cannula is limited due to poor patient tolerance as it dries the nasal mucosa and increases upper- airway resistance(1).HFNC provides precise fractional oxygen delivery and mild positive airway pressure, and it flushes the nasopharyngeal dead space, reducing airway resistance. Thus, an HFNC therapy reduces the patient's work of breathing (2-5).HFNC has been used in several types of respiratory failure in term, preterm infants, children and adults. Some centres have accepted HFNC as an alternative to nasal CPAP. Respiratory failure due to accumulation of fluid, with raised pulmonary vascular resistance, muscle weakness and diaphragmatic fatigue is known to occur in patients undergoing cardiopulmonary bypass. The post extubation period is often challenging and may need use of CPAP or non invasive ventilation(6). In our study we aim to evaluate, whether HFNC in pediatric cardiac surgery patients post-extubation improves $\mathrm{CO} 2$ elimination, improves $\mathrm{PaO} 2$ values and the $\mathrm{PaO} 2 / \mathrm{FiO} 2$ ratio, reduces rates of atelectasis, reintubations and reduces intensive care stay period for the patients, as compared to convention oxygen therapy.

Objectives:

The objective of the study was to evaluate improvement in the following parameters in patients given HFNC post extubation against patients undergoing conventional oxygen therapy:1)PaCO2 levels improvement (CO2 elimination) post extubation.2) Improvement in $\mathrm{PaO} 2$ levels post extubation3) Improvement of the $\mathrm{PaO} 2 / \mathrm{FiO} 2$ ratio post extubation.4) 
Decrease in rates of reintubation5) Decrease in the incidence rate of atelectasis based on chest radiographs comparing immediate post extubation to that at the end of 48 hours6) Decrease in intensive care stay length

\section{Study design:}

A single-centre prospective study was carried out in the Department of Cardiovascular Thoracic surgery in the Lokmanya Tilak Municipal Hospital and Medical College, Mumbai. Inclusion criteria for the study was patients under the age of 5 years undergoing elective cardiac surgery with cardiopulmonary bypass. Patients were classified as cyanotic and acyanotic. The procedures were classified as palliative or curative. Patients having other major concomitant congenital malformations or neuromuscular disease were excluded from the study. Randomisation was carried out using a computer generated software. Definition of post extubation acute respiratory failure TachypneaRR \&gt;50 breaths per minute (\&lt; 1 year old)RR \&gt; 40 breaths per minute (1- 5year old)HypoxemiaSaO2 \&1t;92\% (total repair)SaO2 \&lt;75\% (palliative operation)HypercapniaPaCO2 \&gt; $50 \mathrm{mmHg}$ Increased work of breathing Use of accessory respiratory muscle Procedure: At the end of the surgeries, the patients were shifted to the intensive care unit of the department of Cardiovascular and Thoracic surgery for post-operative monitoring and weaning off the ventilator. All patients were ventilated using the volume guaranteed pressure support system with the aim of supplying tidal volumes of upto $6-8 \mathrm{ml} / \mathrm{kg}$; Positive end expiratory pressure(PEEP) values ranged between $3-5 \mathrm{mmhg}$. As per institutional policy the patients were sedated by a vecuronium and fentanyl infusion till hemodynamic stability was achieved. After hemodynamic stabilisation patients were weaned off mechanical ventilation by the set institutional protocols. First, the patients were shifted to synchronised intermittent mandatory ventilation pressure support (SIMV-PS)mode. Once a satisfactory gas exchange was confirmed on an arterial blood gas analysis, with an adequate respiratory rate, patient was shifted to a pressure support ventilator mode. Thirty minutes later an arterial blood gas analysis was repeated. If the patient was not tachypnoeic and the gas exchange on arterial blood gas analysis was satisfactory, the patient was extubated. Post extubation HFNC or conventional oxygen was administered as per randomisation. In all patients a nasogastric tube was placed and kept open for the first 6 hours. Post extubation the arterial blood gas analysis was done at the end of1, $6,12,18,24,30,36,48$ hours. At the same time the vitals of the patients were also noted. Length of stay in ICU and period and dependence on either conventional oxygen or HFNC was recorded. Optiflow junior nasal cannulae of size M, L, XL were used for the patients. Analysis: All data were collected in Microsoft Excel and were expressed in terms of Mean+/ - standard deviation. Friedman test was used to compare continuous variables between the two groups recorded at regular intervals. Wilcoxon Signed rank test was used for comparing parameters such as rates of reintubation, rates of atelectasis and duration of ICU stay.

\section{Results:}

Of the 128patients operated in the 2 years, who were eligible for the study, 16 patients were excluded as the parents refused consent for participation. A total of 112 patients were enrolled in the study. Of the 112 patients, 56 patients were assigned to the conventional oxygen therapy group and the other 56 were assigned to the HFNC group. Amongst the conventional oxygen therapy group, 31 patients were suffering from cyanotic congenital cardiac anomalies, while 32 in the HFNC were cyanotic. 14 patients in the HFNC group underwent palliative surgery, while 12 in the conventional oxygen therapy group underwent palliation surgery. $\mathrm{PaCO} 2$ values at baseline were comparable and there was no statistically significant difference between the two groups. The post extubation values however showed a significant decrease in the $\mathrm{PaCO} 2$ levels in patients on HFNC therapy at $1,6,12,18,24$, $30,36,48$ hours with p\&1t;0.01 at all times. Diagnosis HFNCCOTTetralogy of Fallot1516VSD1310ASD1416DORV55 HLHS- Glenn Palliation97TAPVC24ASD+VSD88PaO2 levels prior to extubation were comparable in both groups. $\mathrm{PaO} 2$ levels were significantly higher in the HFNC group 1, $6,12,18,24,30,36,48$ hours with $p \& 1 t ; 0.01$ at all times. The $\mathrm{PaO} 2 / \mathrm{FiO} 2$ ratios were comparable in the two groups prior to extubation, but the HFNC group showed a significantly higher ratio at $1,6,12,18,24,30,36,48$ hours with $\mathrm{p} \& 1 \mathrm{t} ; 0.01$ at all times. Of the 56 patients in each group, $5(8.9 \%)$ in conventional oxygen therapy group needed reintubation (4 of respiratory reasons, 1 cardiac reason). While in the HFNC group $3(5.3 \%)$ needed reintubation( 2 respiratory and 1 cardiac reason). 2 patients in the HFNC needed prolonged ICD insertion (5 days) in view of persistent pneumothorax. The mean ICU stay for patients in the conventional oxygen therapy group was 5days (3-9 days). The mean ICU stay in the HFNC group was 3.7days (2-6 days) which was significantly lesser compared to conventional oxygen therapy group (p\&1t;0.01). Both groups demonstrated an equal incidence of atelectasis(5 out of 56) which was appreciated on an Chest Xray done at the end of 48 hours post extubation. Discussion:HFNC has been proposed as the primary modality of therapy for pediatric patients with respiratory distress syndrome and bronchiolitis(7). HFNC has also been propagated in pediatric patients for prevention of post extubation failure(8). HFNC is growing in popularity as an alternative modality of respiratory assistance to CPAP and Non invasive ventilation(9). It was speculated that by flushing the nasopharyngeal space one might be able to reduce the work of breathing thus improving $\mathrm{CO} 2$ elimination process. A study done by $\mathrm{G}$. Testa et al,(10) observed no significant improvement in $\mathrm{PaCO} 2$ values in the HFNC group, though we appreciated significant reduction in the $\mathrm{PaCO} 2$ values. Our study confirmed the improvement in $\mathrm{PaO} 2$ levels and in the $\mathrm{PaO} 2 / \mathrm{FiO} 2$ ratios in the HFNC group which were in line with the study done by G. Testa et al(10), where pediatric surgery patients under the age of 18 months undergoing cardiac surgery were randomly subjected to conventional oxygen therapy or HFNC therapy post-extubation. The study showed remarkable improvement in the $\mathrm{PaO} 2$ and $\mathrm{PaO} 2 / \mathrm{FiO} 2$ levels in both cyanotic and the acyanotic heart disease patients which is in conjunction with our study. A prospective, observational study done bySztrymf B et al(11), compared HFNC with conventional oxygen therapy in adults with acute respiratory failure and showed reduced respiratory rates and improved $\mathrm{PaO} 2$ values in the patients. In their study Holleman-Duray et al.(12) retrospectively analysed premature infants and compared use of HFNC versus CPAP ventilation. They concluded that the patients extubated on HFNC needed fewer days on ventilator and were extubated from high ventillatory rates. Woodhead et al.(13) in a randomized crossover study on premature infants on HFNC versus nonhumidified high flow oxygen observed lower rates of reintubation in HFNC groups. However in our study we did not observe any significant differences in the re-intubation rates between the conventional oxygen therapy group or the HFNC group. Limitations and Short-comings: The period of observation of physiological characteristics was restricted to 48 hours which was relatively short. We could not assess the nasopharyngeal pressures in patients who were subjected to HFNC therapy. In our study we could not comment or differentiate between cardiac or respiratory failure in the participants. Whether the total duration of hospital stay was affected in the participants was not evaluated in our study.

\section{Conclusion:}

HFNC is very useful in improving $\mathrm{CO} 2$ elimination (as evidenced by improvement in $\mathrm{PaCO} 2$ readings) and in improving $\mathrm{PaO} 2$ levels in pediatric patients undergoing cardiac surgery, thus reducing the work and effort of breathing. It can be considered as a safe and better option compared to conventional oxygen therapy in the immediate post-extubation period. References:1) Williams R, Rankin N, Smith T, Galler D, Seakins P. Relationship between the humidity and temperature of inspired gas and 
the function of the airway mucosa. Crit Care Med 1996;24(11):19201929.2) Dewan NA, Bell CW. Effect of low flow and high flow oxygen delivery on exercise tolerance and sensation of dyspnea. A study comparing the transtracheal catheter and nasal prongs.

\section{A RARE CASE OF CORONARY ARTERY FISTULA TO PULMONARY ARTERY}

Dr. Nagendra Nath Murmu, Dr. M. Amaresh Rao

Nizam's Institute of Medical Sciences (NIMS),

Hyderabad

Coronary artery fistula is defined as a rare anomalous connection, in which a coronary artery directly connects to a cardiac chamber or a major vessel. Its incidence is around 0.1 to $1 \%$ in the general population, prevalence in pediatric age group is rare and detection is always tricky as the child presents with only minimal or occasional symptoms. Complications due to the fistula increases with age so early diagnosis and treatment is important. Closure of the fistula is indicatedin patients with myocardial ischemia, large left to right shunt, congestive heart failure or other complications. Herein, we report a 5 years old male child who presented with complain of frequent respiratory tract infection since age of 3 months, cause not detected, coronary artery fistula was suspected onechocardiogram, CT Angiogram confirmed the diagnosis and revealed fistulous connection between right coronary artery and pulmonary artery. The patient was taken for surgery in view of non resolving pulmonary symptoms and further follow up showed that the symptoms resolved andpatient condition improved

\section{A RARE ADULT PRESENTATION OF CONGENITAL PULMONARY AIRWAY MALFORMATION- DOES LOBECTOMY PREVENT MALIGNANT TRANSFORMATION? \\ Atul Abraham, Manoj P Nair \\ Aster Medicity, Kochi}

\section{Introduction:}

Adult presentation of Congenital Pulmonary Airway Malformation (CPAM)- which is an inborn abnormality of the lower respiratory system- is extremely rare. A risk of malignant transformation is one of the reasons for recommending surgical resection.

Case report:

An 18-year-old previously asymptomatic male presented with a sudden onset breathlessness. He was found to have spontaneous pneumothorax on the right and underwent intercostal drain insertion. An HRCT chest was reported as multiple contiguous thin walled bullous lesions involving the right upper lobe. The patient underwent thoracoscopic wedge resection of the diseased lung along with complete surgical pleurectomy. Surprisingly the biopsy showed features of Congenital Pulmonary Airway Malformation (CPAM) with multiple foci of adenocarcinoma in Situ. We discussed the various treatment options with the relatives and proceeded with acompletion lobectomy with mediastinal lymph node clearance. Intra-operatively there were dense adhesions due to the previous pleurodesis. During adhesiolysis, the small middle lobe was lacerated and had to be removed along with the residual upper lobe. Interestingly, the biopsy report showed multiplefoci of CPAM with areas of adenocarcinoma in situ, not only in the residual upperlobe, but also in the middle lobe which was incidentally removed.

Discussion:

The extent of resection in CPAM has traditionally been lobectomy although some authors have reported good results with parenchyma preserving resections. Whether a lobectomy of the involved lobe helps prevent malignant transformation is questionable. These patients have an increased propensity to develop malignancy- not necessarily in the lobe involved by CPAM.

MITRAL VALVE THROMBECTOMY : A NOVEL APPROACH FOR PROSTHETIC MITRAL VALVE THROMBOSIS

Dr Jigar Shah, Dr Anil Jain, Dr Chirag Doshi,

Dr Vivek Wadhawa

U N Mehta institute of cardiology and research center

\section{Objective:}

To evaluate and compare the outcome of Mitral valve thrombectomy versus Redo Mitral Valvere placement for prosthetic mitral valve thrombosis in the early postoperative period.

Methods :

It is a Retrospective observational study of 104 patients admitted forprosthetic mitral valve thrombosis in emergency department of $\mathrm{U} N$ Mehta institute of cardiology and research center from January 2016 to January 2018.Group A ( N-26) patients underwent thrombectomy and Group B ( N-78) patients were treated by Redo mitral valve replacement. Preoperative , intra and postoperative parameters measured to compare the results.

Results :

No statistical difference was found on the basis of gender, age, interval between initial mitral valve replacement (MVR) and redo operation or thrombectomy, anticoagulation status, New York Heart Association (NYHA) functional class, international normalized ratio (INR) level, trans thoracic echocardiography and fluoroscopy. The mean CBP time ( $76.38 \hat{\mathrm{A}} \pm 24.42$ minutes) and cross clamp time ( $40.29 \hat{\mathrm{A}} \pm 16.48$ minutes) was significantly less in group A than group B. Similarly mean ventilation time (14.12 $\hat{\mathrm{A}} \pm 8.37$ hours), Ionotropic support, mean ICU stay

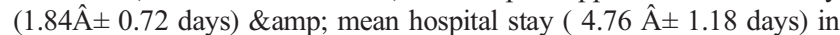
group A was significantly less than in group B. The mortality rate in group A and B was $3.85 \%$ and $11.54 \%$ respectively which was statistically significant.

\section{Conclusion :}

Though prosthetic valve thrombosis after mechanical mitral valve replacement is dreaded complication and night mare for surgeons, if its managed and operated timely with proper surgical planning it will result in wonderful outcome. Thrombectomy is better cost effective option in emergency and it is recommended because of better outcomes in early and late post operative periods.

\section{A CASE REPORT OF MITRAL VALVE PROLAPSE WITH SEVERE MITRAL REGURGITATION WITH HEMOPHILIA TYPE A}

Dr Tribhuvan kumar, Dr Amaresh Rao Mallempati NIMS

We present a case of $32 \mathrm{yr}$ old male who presented with complaints of dyspnoea on exertion, who on evaluation was diagnosed to have Mitral valve prolapse with severe Mitral regurgitation. $\mathrm{He}$ is a known case of Hemophilia type A, consulted with hematologist and managed with factor VIII perioperatively. Patient underwent Mitral Valve Replacement with $31 \mathrm{~mm}$ St Jude rotatable mechanical valve. Postoperative course was uneventful. Patient was discharged after 14 days owing to factor VIII therapy. We present this case because association of valvular heart disease with hemophilia and challenges posed to anticoagulation during and after Mitral Valve Replacement. 
DIFFERENCE IN MEAN TRANS

VALVULAR GRADIENT ACROSS

MONO LEAFLET AND BI LEAFLET

MECHANICAL AORTIC VALVE

OF SIZES 17 AND 19

Vivek Jaunaprasad Dubey

KEM HOSPITAL

\section{Aims and Objectives :}

To Study the statistical difference in mean trans valvular gradient across monoleaflet and bi leaflet mechanical aortic valve of sizes 17 and 19 .

Methods :

50 patients operated for aortic valve replacement with size of 19 and 17 mono leaflet and bi leaflet mechanical aortic valve underwent trans thoracic echocardiography to ascertain the mean gradients after 6 months of their surgeries.

\section{Result :}

Mean trans valvular gradient across mono leaflet size 17 and 19 is 14 and 12. Mean aortic valve gradient across SJ 17 ins 16 and size 19 is14. Mean valve gradient across Sorin 17 and Sorin 19 is 16 and 18. Mean gradient across Cardiamed 17 and 19 is

Conclusions :

After application of unpaired $t$ test, the significance between mean gradients of both the groups, was statiscally not significant.

\section{TRAUMATIC LUNG HERNIATION THROUGH THE CHEST WALL \\ Dr. Ritesh Mate, Dr. Vaibhav shah, Dr. Prashant mishra, Dr. Chaitanya Raut Lokmanya Tilak Municipal General Hospital and Lokmanya Tilak Municipal Medical College, Sion Mumbai}

Traumatic lung herniation through the chest wall is a rare clinical entity. We present a case of significant lung herniation which was related to stab injury in the left anterior chest wall just medical to Mid clavicular line in the 2nd \&amp; 3rd space. The patient was symptomatic with pain and mild dyspnea, patient was vitally stable and chest images revealed a large herniation of the left upper lobe through a defect in the intercostals muscles. Lung herniation is rare but is usually associated with significant trauma or surgery. We report our management for such rare presentation.

\section{PERSISTENT CHYLOTHORAX IN AN ADULT POSSIBLY DUE TO CONGENITAL LYMPHANGECTASIA - CASE REPORT OF A RARE CLINICAL SCENARIO.}

Manoop B,T D Ravikumar

Government medical college, Thiruvanathapuram

\begin{abstract}
Aim:
Though rare in incidence, chylothorax can lead to increased morbidity and mortality. Definitive therapy consists of obliteration and recurrence of chylothorax. Here we present a rare clinical scenario of adult onset congenital lymphangectasia presenting as persistent chylothorax in $23 \mathrm{yr}$ old male patient and its management.

Materials and methods:

$23 \mathrm{yr}$ old male presented with complaints of dyspnoea since $2 \mathrm{yrs}$. Multiple USG guided aspiration was done on left side. MR Lymphangiogram was done could delineate any thoracic duct leak. Lymphoscintigraphy was suggestive of possible chylous pericardial effusion. In view of worsening dyspnoea, Rt posterolateral thoracotomy was done and thoracic duct was lighted. Postoperatively leak persisted, hence
\end{abstract}

thoracic duct embolization was performed using multiple coils and glue covering the location of right hilar chylous duct exit.

Results:

Patient was started on oral diet from POD 3. Minimal chylous drain in initial postoperative day subsided soon and was discharged by POD 6 after removing Rt ICD.

Conclusion:

Sucess of managing massive bilateral chylothorax includes aggressive nutritional support and early surgical intervention when indicated. In the setting of persistent chylothorax even after thoracic duct ligation and all other workup, possibility of lymphangectasia of thoracic, bronchial ducts to be considered. Thoracic duct embolization should always be considered as second step after failure of open/vats duct ligation.

\section{SINGLE CENTRE EXPERIENCE OF PATIENT PROSTHESIS MISMATCH IN AORTIC VALVE REPLACEMENT USING ON-X VALVE}

David Varghese, Alessia Rossi

St Barholomew's hospital

Objectives:

Patient prosthesis mismatch (PPM) is always of concern when performing aortic valve replacement (AVR) in patients with a small aortic annulus and occurs when the effective orifice area (EOA) of the prothesis is too small in relation to the patients body surface area. PPM has been reported to increase perioperative mortality and reduce post-operative survival in patients undergoing aortic valve replacement. The aim of this study is to determine the incidence of PPM in a series of 54 consecutive patients undergoing isolated AVR with the $\mathrm{ON}-\mathrm{X}$ valve. We used the indexed effective orifice area $(\mathrm{cm} 2 / \mathrm{m} 2)$ to identify cases of PPM. Severe PPM (effective orifice area index\&lt;0.65 $(\mathrm{cm} 2 / \mathrm{m} 2)$ ), moderate PPM (effective orifice area index 0.65 to $0.85(\mathrm{~cm} 2 / \mathrm{m} 2)$ ), and absent PPM (effective orifice area index\&gt;0.85 (cm2/m2)).

\section{Methods:}

54 patients underwent isolated aortic valve replacement with $\mathrm{ON}-\mathrm{X}$ valve between June 2017 to February 2019. Using the manufacturerâ $€^{\mathrm{TM}_{\mathrm{S}}}$ safety guide 7 patients that had mild PPM were identified. There were no cases of severe PPM.

Results:

There were 3 patients that had a size $21 \mathrm{~mm}$ valve implanted, all male and 4 that had a size $19 \mathrm{~mm}$ valve implanted, all female. Median age was 60 (range 47 to 64). Median Euroscore II was 1.8 (range 0.6 to 13.0). Average CPB time was 91 minutes $+/-23$ minutes. Average cross clamp time was 74 minutes $+/-10$ minutes. The average post-operative peak gradient across the valve was $33 \mathrm{mmHg}$.

\section{Conclusion:}

The ON-X valve provides excellent hemodynamic benefits even in cases of mild PPM. We would suggest that during the valve time out the iEOA to be mentioned in cases where a size 19 or $21 \mathrm{~mm}$ is to be implanted. Whilst aortic root enlargement procedures have now fallen out of favor, authors such as Hopkins et al have shown that aortic root enlargement procedures can be performed with no undue technique related surgical deaths or complications compared to isolated aortic valve replacement.

\section{A MODIFIED TECHNIQUE IN CORONARY REIMPLANTATION FOR ALCAPA REPAIR}

Dr Nikhil Dixit, Dr Amaresh Rao, Dr K Madhusudhan, Dr B Kaladhar, Dr PSS Gopal Nizam's Institute of Medical Sciences, Hyderabad

Anomalous left coronary artery from the pulmonary artery (ALCAPA) is a rare congenital anomaly The majority of ALCAPA cases usually present 
in early infancy. With the decrease in pulmonary vascular resistance during the first few weeks of life, myocardial ischemia and/or infarction may occur. The clinical features depend on the degree of coronary artery collateral development. Due to the lack of specificity in its clinical manifestations, ALCAPA might be misdiagnosed as dilated cardiomyopathy, endocardial fibroelastosis, or mitral valve lesions. The early mortality rate can be as high as $90 \%$ if ALCAPA is not diagnosed and treated in a timely manner. For the surgical management of ALCAPA, there have been various techniques that reduce the tension and kinking of the coronary artery during reimplantation to the aorta. We present a case of a 10 year old female who presented with ALCAPA and dilated RCA and conal branches. She underwent coronary reimplantation with a modified technique (translocation of the left coronary ostia with posterior wall of PA as a conduit to Aorta and pericardial patch augmentation of PA) of repair. Post operative period was uneventful and patient recovered well

\section{PUMP-ASSISTED CABG: A BRIDGE BETWEEN ON PUMP CABG AND OFF PUMP CABG}

Avinash Prakash

Kem Hospital, Mumbai

\section{Aim/Objective(s):}

To assess the outcome of a hybrid approach-Pump-Assisted CABG (PAD-CAB)- with the aid of $\mathrm{CPB}$ without aortic cross-clamping and cardioplegic arrest.

Material and method:

Between November 2017 andNovember2019, 30 PAD-CAB procedures were performed by post-mch Residents. The $\mathrm{PAD}-\mathrm{CAB}$ procedures were achieved with standard CPB via sternotomy under normothermic conditions. Outcome measures included hospital mortality and specific major adverse events (MAE) benchmarked against the Society of Thoracic Surgeons (STS) database. The number of bypass grafts, status of the case, specific patient factors, and postoperative length of stay (LOS) were also assessed.

Result(s):

There were 24 male (80\%) and $6(20 \%)$ female patients. The mean age was 50 years (range: 38 to 72 years). The mean ejection fraction (EF) was $50 \%$ (range: 20 to $65 \%$ ) with 6 cases (20\%) having an EF\&lt;40\%. 24 (80\%)were non-emergent with $6(20 \%)$ classified as salvage. The average of number of bypass grafts was 3.24 (range: 1 to 5). The postoperative LOS averaged 7.5 days with a median of 6 days. There were 1 hospital deaths (3.3\%). Major adverse events were: 1 deep SWI (3.3\%), 0 CVAs $(0.95 \%)$ Conclusion:

PAD-CAB is a safe bridging step between ON PUMP CABG \&amp; $\mathrm{OPCAB}$, and can be safely performed with shorter learning curve by residents in a teaching institute. The PAD-CAB technique takes advantage of the circulatory stability achieved with $\mathrm{CPB}$ assistance and eliminates the potential risks associated with aortic cross-clamping and cardioplegic arrest.

\section{A RETROSPECTIVE ANALYSIS OF REOPERATIVE VALVE SURGERY IN THE LAST FIVE YEARS. (2014-2018) \\ Dorendro Thingujam}

Christian Medical College, Vellore

\section{Aim:}

The aim of this study was to evaluate the overall outcome of patients undergoing reoperative/redo valve surgery in the last five years at our institution.

Materials:

72 patients (males $=45$, females $=27$ ) underwent redo valve replacement with either mechanical $(n=63)$ or bioprosthetic $(n=7)$ valves or repair $(n=2)$ between January 2014 and December 2018.

Results:

Median age of patients was 41 years (range 18 to 75 years). 3 patients were undergoing valve replacement for the third time. Time to reoperation varied from 4 months to 31 years. Indications include prosthetic valve endocarditis, para-prosthetic leak, structural valve degeneration, prosthetic valve thrombosis, prosthetic valve dehiscence and prosthetic valve block. 67 patients underwent routine surgery whereas 5 patients underwent emergency operation. Mortality rate in hospital was 4 . There were 3 patients that required re-operation during hospital stay.

Conclusion:

With the increase in number of cardiac operations being done worldwide, the number of redo surgery is also increasing steadily. The study also highlights the major causes of redo in valve surgeries. It also shows that redo surgery can safely be done with acceptable mortality limits.

\section{RARE PRESENTATION OF COMMON CARDIAC DISEASE OF INDIAN POPULATION - SUBMITRAL ANEURYSM OF RHEUMATIC AETIOLOGY}

Dr. A.S. Mughilan, Karthikeyan B MS MCh, Meenakshi sundaram MS MCh,

Muthukumar M MS MCh, Marvin Manoah

Baylis MS MCh, Rathinavel A MS MCh,

Department of Cardiovascular and Thoracic surgery, Madurai medical college.

\section{Introduction:}

Submitral aneurysm of the left ventricle (LV) though relatively unknown is a widely recognized cardiac pathology of varied aetiology such as inflammation, infection, or traumatic illness which occurs most commonly among the black population. Patients with submitral aneurysm exhibit varied clinical manifestations and are reported to have poor surgical outcome. The infectious illnesses mentioned most often as possible causes of these types of aneurysms are syphilis and tuberculosis.

\section{Case report:}

We report here a patient - 13-year-old female child presented with dyspnoea on exertion, orthopnea and PND. Echo cardiography was done which revealed a submitral aneurysm below the posterior mitral leaflet with dilated LA, mild to moderate Mitral regurgitation and moderate pulmonary hypertension. Cardiac CT confirmed the echo findings. Then patient has been planned for surgical repair .Patient underwent excision of aneurysm with closure of defects and mitral valve replacement with 25 TTK mitral valve. Aneurysm wall sent for Histopathological examination came as acute vegetation of acute rheumatic fever.

\section{Conclusion:}

Submitral aneurysm is rare condition which can be primary or secondary. Even though the aetiology of the condition is thought to be congenital, Takayasu's arteritis and tuberculous pericarditis has been reported. Definitive treatment is surgical. Rheumatic etiology of submitral aneurysm is rarest of rare. Hence, we would like to present this case in this forum. Mortality is high in un-operated cases and operative repair under cardiopulmonary bypass is the most appropriate management.

AUTOLOGOUS PERICARDIAL AORTIC VALVE RECONSTRUCTION: EARLY EXPERIENCE IN COMPLEX CARDIAC AND AORTIC ANATOMY

Jeeva Vijayan, Prasanna Simha Mohan Rao, Rakesh Naik Lachma

Sri Jayadeva Institute of Cardiovascular Sciences and Research 


\section{Background/ aim:}

Autologous pericardial aortic valve reconstruction was originally described by Ozaki et al. It is finding acceptance as a safe, efficacious method for treating Aortic valve diseases. We have done 75 cases in our Institute. Initial good results gave us courage to extend this surgery to patients with complex cardiac /aortic anatomy and to those requiring multiple cardiac procedures simultaneously. We are sharing our experience of such complex procedures.

Materials and methods:

At our institute, 75 autologous pericardial aortic valve reconstructions were performed from 2016 till date. All the procedures were performed through lower midline hemisternotomy except in that requiring ascending root replacement. The complex aortic valve reconstructions that have been performed included the following: 31 with associated Mitral valve repairs +/- Tricuspid valve repairs,19 Bicuspid Aortic Valves,1 Unicuspid aortic valve, 1 Bicuspid aortic valve with Ascending Aortoplasty,1 Bicuspid aortic valve with Ascending Aortic Replacement, 1 tricuspid aortic valve with Ascending Aortic Replacement, 1 with Manouguian aortic root widening, 1 with Mitral Valve replacement with Bioprosthetic valve, $1 \mathrm{CABG}$ and 1 with Aortic repair with type B Aortic Dissection.

Results:

All the complex aortic valve reconstructions had excellent outcomes. They also had the known benefits of autologous pericardial aortic valve reconstruction like lower aortic valve pressure gradients, bigger orifice area, better valve hemodynamic etc. None of them had thrombohaemorrhagic complications.

Conclusion:

Autologous pericardial aortic valve reconstruction is a feasible option in complex cardiac and aortic anatomy with favourable outcomes.

\section{VENO-ARTERIAL ECMO AS A \\ RESCUE THERAPY IN POST \\ INFARCT VENTRICULAR RUPTURE}

Shobhit Saurav, Pankaj Gupta, Rajneesh Malhotra

Max super speciality hospital, Saket, New Delhi

Outcomes of ventricular septal rupture as a complication of acute myocardial infarction are extremely poor evidenced by an in hospital mortality rate of over $45 \%$ in surgically treated patients and over $90 \%$ in patients managed medically. Extracorporeal membrane oxygenation is a complex rescue therapy utilised to provide circulatory and/or respiratory support to critically ill patients who have failed maximal conventional therapy. Non surgical treatment of post MI VSR has an early mortality of over $90 \%$ and hence surgical repair might have a better outcome. Here we describe a case treated successfully with Veno-Arterial extracorporeal membrane oxygenation support pre-operatively and post-operatively as a bridge to recovery in a young patient with post myocardial infarction induced large ventricular septal rupture.

\section{CHRONIC THROMBOEMBOLISM WITH PULMONARY HYPERTENSION}

Solomon

Christian Medical College

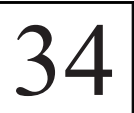

\section{Introduction:}

Chronic thromboembolic pulmonary hypertension occurs in 3 to 30 individuals permillion general population per year and has been shown to be a long term complication of pulmonary embolism with a cumulative incidence of $0.1 \% 9.1 \%$ within two years after a symptomatic event.

Background:
Chronic thromboembolic pulmonary hypertension is caused by chronic obstruction of major pulmonary arteries following pulmonary embolism. It appears to be caused primarily byvenous pulmonary thromboembolism as opposed to primary pulmonary vascular in situ thrombosis. Prothrombotic factors such as inadequate anticoagulation ,a large thrombus mass and residual thrombus and recurrences may contribute the development of disease. Simplistically Chronic thromboembolic pulmonary hypertension as a disease caused by obliteration of central pulmonary arteries by pulmonary emboli.

Case report:

A 22 year old female presents with breathlessness for 2 years and hemoptysisfor same duration. She was previously diagnosed as having sickle cell trait. Clinical examination shows systolic murmur over pulmonary area with jugular venous distension. CTpulmonary angiogram showed -large thrombus seen in right main pulmonary artery measuring $22 * 23 \mathrm{~mm}$ with thrombus extending into right lower lobar segment and subsegmental vessels. Floating thrombus seen in the Right ventricular cavity measuring $19 * 15$ $\mathrm{mm}$, cardiomegaly with dilated Right atrium, right ventricle and enlarged superior vena cava, inferior vena cava seen. Midline sternotomy with right ventricle thrombectomy done. She had no problem in the postoperative period and warfarin was started.

Conclusion:

Early diagnosis and treatment of disorder is important as surgery is the treatment of choice. Pulmonary angiography in anteroposterior and lateral projection confirms diagnosis. Keywords: Prothrombotic factors, sickle cell trait, pulmonary angiography.

\section{A RETROSPECTIVE STUDY OF SURGICALLY TREATED CHRONIC THROMBOEMBOLISM WITH PULMONARY HYPERTENSIONOVER A PERIOD OF LAST ONE AND HALF YEARS. \\ Solomon \\ Christian Medical College,}

\section{Introduction:}

Study of thromboembolic pulmonary hypertension is made interesting by varied number of etiologies, making definitive diagnosis difficult. Recent improvement in diagnostic techniques, advances in surgical and postoperative management have improved the total outcome of the patient. Aims and objectives:

To study retrospectively all the cases operated on for thromboembolic pulmonary hypertension in our Department to analyse retrospectively the incidence, their clinical presentation methods employed in diagnosis and surgical intervention done.

Materials and methods:

Two cases operated on for thromboembolic pulmonary hypertension, at Christian Medical College Hospital, Vellore for the last one and half years were analysedretrospectively.

\section{Results:}

Among the two patients -1 male and 1 female. Clinical examination was crucial in this disorder for diagnosis. Patients usually presents late as physical signs and symptoms in early disorder are generally absent. Only in the later disease stage- Right sided heart dysfunction detected. Surgery is the treatment of choice. After surgery most patients exhibit hemodynamics that are almost normal and experience substantial relief from symptom.

Conclusion:

Chronic thromboembolic pulmonary hypertension is common subset of pulmonary hypertension that is curable by surgery. This disorder occurs equally in both sexes and age groups can be affected, yet median age of patient is 63 years. Pulmonary angiography confirms the diagnosis. Surgery is the treatment of choice 
ASD WITH PULMONARY

STENOSIS REPAIR VIA RIGHT

MINI-THORACOTOMY APPROACH

WITH CONVENTIONAL CENTRAL

CANNULATION : CASE REPORT

Dr Parth Vaghela, Dr. Anil Sharma,

Dr. Sunil Dixit, Dr. Mohit Sharma

Senior Resident, SMS Medical College, Jaipur

\section{Introduction:}

At present thoracotomy with femoro-femoral bypass is established approach for minimally invasive open heart surgeries but thoracotomy with conventional cannulation is yet to be established. A 20-year-old young female referred to our department with ASD and severe PS. We performed intra-cardiac repair of ASD with dacron patch and PS was relieved via pulmonary artery and RVOT without femoro-femoral by-pass without any specifically designed equipment. There was no procedure related complication.

\section{THORACOTOMY APPROACH WITHOUT FEMORO-FEMORAL BY-PASS FOR DOUBLE VALVE REPLACEMENT: OUR EXPERIENCE \\ Dr Anil Sharma, Dr Sunil Dixit, \\ Dr. Mohit Sharma, Dr Dhruv Sharma, \\ Dr K.K. Mahawar \\ SMS Medical College, Jaipur}

\section{Background:}

Double valve replacement (DVR) is usually done through median sternotomy. However, right anterolateral thoracotomy without femorofemoral by-pass has been developed at our center with conventional instruments with many advantages.

Aim:

The aim of this study was to analyze the feasibility and results of right anterolateral thoracotomy without groin incision for DVR.

\section{Patients and Methods:}

This was a prospective study conducted on during the period from June 2014 to June 2019 in S.M.S. Medical College, Jaipur. This study consists of 56 patients who had a concomitant mitral and aortic valve disease and were subjected to DVR.

\section{Results:}

This was a prospective study in our center. Among 56 cases 32 were females and 24 were male. Age ranges from 18 to 56 years. Most patients were in NYHA class III/IV and postoperatively in 2 months there was significant improvement to class I/II. Rheumatic heart disease was the pathology in $80 \%$ and degenerative in $20 \%$. The average size of incision was $6-8 \mathrm{~cm}$. The average cross clamp time (ACC) was $83 \mathrm{~min}$ (Range 58 to $112 \mathrm{mins}$ ) and average cardiopulmonary bypass time (CPB) was $1 \mathrm{hr} 36$ mins. Average total surgical time was 2 hrs 56 mins. Postoperative ICU stay was 36-48hrs and postoperative length of stay was 5-6 days. We used intercostal nerve block with Bupivacaine so almost all patients have less requirements of pain killers. All patients weaned off from ventilator within $12 \mathrm{hrs}$. No patient was reexplored for bleeding. Average bleeding was less than $400 \mathrm{ml}$. 16 Patients required Amiodarone infusion for fast atrial fibrillation.

\section{Conclusion:}

DVR via thoracotomy appears to be associated with faster recovery, less pain and early mobilisation, good cosmetic satisfaction, less blood transfusion and related hazards, early discharge and shorter hospital stay and cost.
ASCENDING AORTIC ORIGIN OF A

BRANCH PULMONARY

ARTERYÂ€"SURGICAL MANAGEMENT

AND LONG-TERM OUTCOME

Dr. Herin Patel, Dr. Kartik Patel, Dr. Trushar Gajjar,

Dr Amit Mishra, Dr pankaj Garg, Dr Rahul Kumar

UN Mehta Institute of Cardiology

and Research Centre

\section{Introduction:}

Anomalous origin of pulmonary artery is rare anomaly where one of the pulmonary branch artery arise from ascending aorta. Most of these patients are diagnosed in early infancy and early surgical repair is indicated in such cases. But in India, delayed presentation beyond 6 months is common.

Aim and objective:

To demonstrate our outcomes of 16 such patients who had late presentation and treated surgically with excellent post op outcomes.

Material and methods:

Retrospective study of 16 patients from 2011-2018 was done with detailed preoperative, intraoperative and post-operative evaluations. . Results were reported as mean $\hat{\mathrm{A}} \pm \mathrm{SD}$. Follow-up records were specifically reviewed for surgical complications and any re-interventions.

\section{Results:}

16 patients were included. Mean age was $7.6 \hat{\mathrm{A}} \pm 5.7$ (range of 2 to 22 months). $93.75 \%$ patients had anomalous right pulmonary artery and $6.25 \%$ patients had left anomalous pulmonary artery origin. All patients had primary closure of aorta with direct implantation of anomalous pulmonary artery onto the side of MPA. Mean length of stay in ICU and hospital was 4 and 8.56 days. In follow up, the mean systolic pressure in branch pulmonary artery was 25 . The mortality rate was $18.75 \%$.

\section{Conclusion:}

Anomalous origin of branch pulmonary artery results in exposure of pulmonary circulation to pressure and volume overload. Early diagnosis and prompt surgical treatment results in excellent survival and avoids development of pulmonary vascular obstructive disease. In our experience, patients had delayed presentation post 6 months and post surgically had somewhat higher early mortality rate. But once patients survived early post op period, they had equivalent survival to those patients who were diagnosed and treated surgically early in infancy.

\section{TET CANAL REPAIR- 6 YEARS EXPERIENCE}

Sujoy Chatterjee, Srirup Chatterjee,

Rajarshi Ghosh, Santosh Kumar Pandey,

Lakshmi Sankhayan, Nikhil Bansal, Satyajit Bose

The Mission Hospital, Durgapur

\section{Introduction:}

Complete AVSD with TOF is a very rare congenital anomaly. Long term experience of surgical management of this anomaly is sparse. We are reporting our experience of 28 patients who were operated over a period of 6 years.

Material and methods:

Between a period from May 2013 to April 201928 patients were operated for tet cannal. 5 patients were previously shunted for small branch PAs. 20 of these had Down's syndrome. Mean age at operation was 4.3 years ( range 1 year to 12 years). All patients were operated by 2 patch technique, dacron patch for VSD and unfixed pericardium for OP-ASD. TAP was required for 18 patients out of which 4 patients required RV-PA conduit.

Results:

Mean follow was 2.3 years (range 5 years to 6 months). There were 4 deaths out of which 2 died with 30 days of surgery due to intractable low 
cardoac output and severe pulmonary haemorrahge. 2 patients were lost to follow up after 6 months of surgery. On discharge there were no residual ASD or VSD in any of the patients, most had mild right and left AVV regurgitation. Mean RVOT gradient was $15 \mathrm{~mm}$ of hg (range $12 \mathrm{~mm}$ of hg to $20 \mathrm{~mm}$ of hg). None of our patients required re operation so far.

\section{Conclusion:}

Though rare, when appropriately treated patients with tet canal have excellent survival, at least over this follow up period.

\section{IS TRICUSPID VALVE REPAIR} ALWAYS NECESSARY TO PREVENT THE PROGRESSION OF TRICUSPID REGURGITATION FOLLOWING

MITRAL OR DOUBLE VALVE REPLACEMENT IN PATIENTS

WITH RHEUMATIC HEART DISEASE ? AN AMBISPECTIVE OBSERVATIONAL

\section{STUDY}

Hemachandren Munuswamy,

Duvuru Ram, Palapathi Sairam,

Ananth Kumar Sivanesan, Ajith Ananthakrishna

pillai, Saichandran Bethela vedhagiri

Jawaharlal Institute of Postgraduate Medical

Education and Research (JIPMER)

\section{Objectives:}

Among patients with rheumatic heart disease (RHD) undergoing mitral or double valve replacement (MVR/DVR), to compare the progression of functional tricuspid regurgitation (TR) and quality of life (QOL) between those with and without tricuspid valve (TV) repair.

Methods:

It was an ambispective observational study conducted on 240 RHD patients who underwent MVR/DVR with or without TV repair from January 2008 to November 2019. (Jip/Sur/IEC/SCm/ 30/2016/31). Those patients with organic TR and ischemic etiology were excluded. Demographic details, disease characteristics, preoperative details, type of surgery, intraoperative details, prosthetic valve function, ECHO findings, preoperative and follow up TR severity, postoperative QOL using Minnesota Living with Heart Failure questionnaire were analysed.

Results:

Mean age of patients was $37.08 \hat{\mathrm{A}} \pm 10.80$ years. MVR and DVR was performed in 182 and 58 patients respectively. Severe PAH was present in $57.7 \%$ of cases preoperatively and in $5.8 \%$ at follow up $(p=0.0001)$. Preoperative TR was present in $84.4 \%$ patients with moderate and severe TR in $26.1 \%$ and $41.5 \%$ of cases respectively. DeVegas annuloplasty was done in $42.2 \%$ of patients with moderate and severe TR. At follow up, progresssion of TR was present in $15 \%$ vs. $28.5 \%$ among those with and without concomitant TV repair $(\mathrm{p}=0.05)$. Among those 78 patients with severe TR, there was no significant difference in improvement in TR severity with or without DeVegas repair $(86 \%$ vs $71.8 \%, \mathrm{p}=0.09)$. QOL was good in $51.6 \%$, moderate in $47.6 \%$ and poor in $0.8 \%$ of patients at follow-up. There was no significant difference in the QOL between those with or without progression of TR $(\mathrm{p}=1.000)$.

Conclusion:

Progression of TR and QOL of patients with TR at follow up remained same irrespective of TV repair. Improvement in grade of TR occurs irrespective of TV repair as evidenced by significant reduction in proportion of patients with severe PAH. Hence, not all patients with moderate and severe TR require valve repair and decision to repair needs to be individualized based on intraoperative assessment of valve.
OPD MANAGEMENT OF

PERIPHERAL ARTERIAL

DISEASE PATIENTS IN A TERTIARY

CARE HOSPITAL: DOES ASPIRIN

AND CILOSTAZOL HELP - A PILOT

STUDY FROM A HIGH VOLUME

TERTIARY CARE CENTRE.

Dr Nadeem Ul Nazeer

Department of CTVS, SKIMS Institute,

Srinagar, J and K, 190011

\section{Background:}

Cilostazol is the first-line drug for peripheral arterial disease (PAD) because it improves the symptoms and quality of life. The treatment of PAD involves the prevention of cardiovascular events plus relief of symptoms including intermittent claudication (IC). The objective of this study was to evaluate the efficacy of aspirin and cilostazol in patients suffering from peripheral arterial disease (PAD).

Methods:

Patients were evaluated on outpatient department (OPD) basis. Meticulous history was followed by clinical examination and measurement of ankle-brachial index for diagnosing PAD. The patients also underwent duplex imaging, computed tomography angiography or magnetic resonance angiography. The treatment includes lifestyle modification in the form of smoking cessation and exercise; management of atherosclerotic risk factors, including anti-platelets, statins, drugs for hypertension; and agents to improve walking distance, such as cilostazol and enalapril.

Results:

The mean age of the patients was $47 \hat{\mathrm{A}} \pm 6.2$ years. History of smoking, either past or current, was present in $91 \%$ of patients. History of ischemic heart disease was present in $2.17 \%$, while $26.08 \%$ patients were diabetic. Aspirin and cilostazol were prescribed after the diagnosis was made. Symptomatically, all the patients improved. Walking time was improved in all the patients.

\section{Conclusion:}

The safety profile of aspirin and cilostazol in patients with PAD appears to be acceptable. In addition to risk factor management, treatment with cilostazol should be considered in patients of PAD.

\section{DYSFUNCTION AFTER \\ MITRAL VALVE SURGERY - BOON FOR CARDIAC SURGEONS}

DR Divyesh Rathod,

DR Vivek Wadhawa, DR Chirag

Doshi, DR Jigar Shah

U N Mehta Institute of

Cardiology and

Research Center

Aims :

Left ventricular (LV) dysfunction is the first cause of early as well as late mortality after mitral valve replacement surgery. In this retrospective analysis, we studied the association between preoperative echocardiographic LV measures and occurrence of LV dysfunction after mitral valve replacement (MVR).

Methods and results :

Between January 2017 and august 2018 , 563 consecutive patients underwent MVR for severe mitral stenosis and or regurgitation due to rheumatic affection in our institution. Echocardiography was performed preoperatively and post operatively at early post operative period after surgery in patients who represented the study population. Cardiac events were recorded during follow-up. LV ejection fraction (EF) decreased from $68+9 \%$ before surgery to $59+9 \%$ post-operatively $(\mathrm{P}, 0.001)$. Preoperative EF ,64\% and LV end-systolic diameter (ESD) â\%o $¥ 37 \mathrm{~mm}$ 
were the best cut-off values for the prediction of post-operative LV dysfunction (EF, 50\%). On the basis of a combined analysis, the occurrence of post-operative LV dysfunction was $9 \%$ when EF was â\%o $¥ 4 \%$ and LVESD ,37 mm, 21\% with EF ,64\% or LVESD â\%o $37 \mathrm{~mm}$, and $33 \%$ with EF ,64\% and LVESD â\%o $¥ 37 \mathrm{~mm}$ (P for trend ,0.001). The combined variable $\mathrm{EF}, 64 \%$ and LVESD â\%o $37 \mathrm{~mm}$ added incremental prognostic value to the multivariable regression model ( $\mathrm{P} \hat{\mathrm{A}}^{1 / 4}$ 0.001).

Conclusion :

Simple preoperative echocardiography measures allow the prediction of LV dysfunction after MVR in patients with leaflet prolapse. Patients with preoperative $\mathrm{EF}$ a $\% ¥ 64 \%$ and LVESD, $37 \mathrm{~mm}$ incur relatively low risk of post-operative LV dysfunction.

\section{COR-TRIATRIUM DEXTER, ATRIAL SEPTAL DEFECT AND \\ PARTIAL ANOMALOUS \\ PULMONARY VENOUS CONNECTION \\ IN A 35-YEAR-OLD FEMALE}

Dr. Utkarsh Sanjay Sanghavi, Dr. Hiremath CS,

Dr. Shekhar Rao

Sri Sathya Sai Institute of Higher Medical

Sciences, Bengaluru

\section{Introduction:}

Cor-triatriatum dexter is a rare congenital heart anomaly in which a membrane divides the right atrium into 2 chambers and exists either in isolated classical form or may be associated with simple to complex congenital cardiac anomalies.

\section{Case report:}

A 35-year old female presented with complaints of shortness of breath and atypical chest pain restricting her daily activities for 12 months. Her second heart sound was wide and fixed split and an ejection systolic murmur was heard in the pulmonary area. Transthoracic echocardiography (TTE) showed a membrane in the RA with OSASD, dilated RA and RV and normal biventricular function. CT cardiac showed Cor triatrium dexter with membrane running from the level of Thebesian valve of coronary sinus and eustachian valve of IVC inferiorly and laterally to the lower part of crista terminalis. The right upper and lower PVs draining into RA, large OSASD and a small SVASD. Via median sternotomy, CPB was established with aortic and bicaval cannulation. Membrane was resected, ASDs were closed with baffling of pulmonary veins into LA using autologous untreated pericardial patch. Post-operatively patient was discharged on POD 5. TTE at discharge showed no residual membrane, ASD patch intact and all pulmonary veins draining into LA, IVC draining into RA and normal biventricular function.

\section{Conclusion:}

Cor-triatrium dexter is rare in adults. Preoperative imaging with echocardiography and CT scan is essential for making correct therapeutic decisions. In patients with concomitant heart anomalies, open-heart surgery remains the gold standard in the treatment of cor-triatriatum dexter.

\section{Bilateral lower limb perfusion for management of limb ischaemia during ECMO}

S. Sabiha Nigar, Ketana. V. Rajasekhar

Rao 1, Dr Abhishek manmohan

sahu .1 Muntimadugu T.Babu,1

Madhusudan R. Jaju 2

1.Department of cardiothoracic surgery, C,

Nampally, Hyderabad ARE Hospital2.

Department of Crtical care CARE Hospital,

Nampally, Hyderabad

\section{Introduction:}

Limb ischaemia in patients undergoing extra corporeal membrane oxygenation [ECMO] is a common problem. To prevent ischaemia in the limb with arterial cannula a distal perfusion cannula is placed in Superficial femoral artery [SFA] prior to placement of femoral arterial cannula in common femoral artery [CFA]. In our patient after placement of distal perfusion cannula in the right SFA ,Right CFA lcannulation could not be done successfully and the attempt to cannulation resulted femoral artery injury and limb iscahema, The limb ischaemia was treated by perfusion through distal SFA perfusion cannula which was placed before.

Case report:

$67 \mathrm{yr}$ old male patient was admitted with pulmonary edema, severe $\mathrm{lv}$ dysfunction, ARDS and shock. It was decided to place arterio venous ECMO for him inview of severe desaturation and hypotension inspite of high supports. Right SFA distal perfusion cannula was placed prior to placement of CFA cannula . While unsuccessful attempt was made to cannulate right CFA femoral artery flow was impaired and resulted in ischaemia of leg which was treated with perfusion through SFA cannula. Ecmo was performed with left femoral arterial and rt femoral venous cannuala. Distal [ SFA] perfusion cannula was used for perfusion of left leg to prevent ischaemia. Discussion:In case of injury to femoral artery and ischaemia while attempting to cannulate femoral artery for ECMO distal perfusion through SFA could be used to deal with leg ischemia. Key words Limb ischaemia, distal perfusion, Ecmo.

\section{SURGICAL OUTCOME OF TOTAL ANOMALOUS PULMONARY VENOUS CONNECTION (TAPVC) REPAIR}

Dr. Vaibhav Chugh (MCh 2nd year),

Dr Dhananjay Bansal (Assistant Prof),

Dr Narender Singh Jhajhria (Prof),

Dr Vijay Grover (Prof \& HOD),

Dr Vijay Kr Gupta (Prof)

Abvims and Dr. RML Hospital, New Delhi

\section{Objectives:}

Total anomalous pulmonary venous connection (TAPVC) is a rarecyanotic congenital heart defect. This study aimed to evaluate the outcome of TAPVC correction in our institute.

\section{Methods:}

Between August 2011 and July 2019, 35 TAPVC patients underwent surgical repair in our department. The medical records of the patients were retrospectively reviewed. Out of these 35 patients, 20 cases $(57.14 \%)$ were infants(\&lt; $1 \mathrm{yr})$ and rest $(42.86 \%)$ were of paediatricage group (1-14 yrs). The anatomic TAPVC subtypes were: 22 supracardiac (62.8\%), 5 cardiac (14.3\%), 1 infracardiac $(2.9 \%)$ and 7 mixed $(20 \%)$. Preoperative Pulmonary venous obstruction was present in 5 patients $(14.3 \%)$. Emergency operation was conducted in these obstructed patients. The follow-up for the entire study ranged from 4 months to 5 years (mean 3.4 years).

\section{Results:}

Mortality occurred in 4 patients (11.4\%), within 24 hrs of surgery. All four were of less than $1 \mathrm{yr}$ age and three among these had pre-operative pulmonary venous obstruction. No any major morbidity was noted.

Conclusion:

Surgical treatment of TAPVC carried satisfactory results with low operative risk. The younger age, preoperative pulmonary venous obstruction, the timing of the emergency operation affect prognosis. Overall, survival has significantly improved over the past decades through better perioperative management and evolution of surgical approaches to minimize postrepair pulmonary vein stenosis, which persists as a major determinant of long-term outcomes. 
MEDIASTINAL MASS WITH

PHPT - TWO INTERESTING CASES

Dr. Sampaul Richard, Dr. Sreekar, Dr. Suresh

St. Johns medical college and hospital

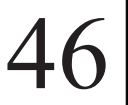

\section{Scenario 1 -}

Aim :

To present a Thymic carcinoid that occured synchronously with a giant Parathyroid adenomain a 37 year old patient - ? MEN 1

Material and method :

Herein we report an unusual case of a 37 year old patient who presented with history of chest pain and back pain on and off for 2 years. The patient on work up was diagnosed with a left mediastinal mass on CT imaging along with nodules in the thyroid gland. Pre-op evaluation showed a raised Calcium and PTH levels. Its evaluation, diagnosis and management will be discussed.

Result:

Patient underwent Sternotomy + thoracotomy with combined Left parathyroidectomy. The PTH levels fell drastically post-surgery.

Conclusion :

Primary neuroendocrine tumors of the mediastinum are very rare and only few cases are associated with parathyroid adenoma. Such unusual cases should be thoroughly investigated to rule out genetic syndromes and other tumors.

\section{Scenario 2 -}

Aim :

Ectopic Mediastinal parathyroid adenoma and PHPT in a 31 year old female

\section{Material and method :}

We describe a case of 31 year old female with complaints of vomiting, abdominal pain for 10 days with a past history of acute pancreatitis and renal calculi. Blood reports revealing persistent high levels of calcium and PTH, the suspicion of hyperparathyroidism was raised warranting the need for PET scan. The scan showed focal tracer uptake in the anterior mediastinum, behind manubrium suggesting an ectopic parathyroid adenoma. Evaluation, management and its outcome will be discussed .

\section{Result :}

The tumor was surgically excised .

Conclusion :

This case helps bring to light the need to consider Ectopic parathyroid adenoma in case of persistent Symptomatic hypercalcemia and absence of neck swelling. We report this case for its unusual scenario and rarity.

\section{CASE OF SMALL AORTIC \\ ROOT IN A PATIENT WITH \\ DOUBLE VALVE DISEASE OF \\ RHEUMATIC ETIOLOGY}

Dr. Dinesh kumar. R, Dr. Anagha Tulsi A.,

Dr. Hiremath C.S, Dr. Shekhar Rao.

Sri Sathya Sai Institute of higher medical sciences

\section{Introduction:}

Early rheumatic valve involvement with late presentation is more common in developing countries. In late presenters, multiple valve involvements with severe fibrosis are more common. This fibrosis causes narrowing of the annuli likely to cause PPM particularly of the aortic valve. We report a case of rheumatic mitral and aortic stenosis with a small aortic root

\section{Case Report:}

A 43-year female with BSA of $2.0 \mathrm{~m} 2$, presented with dyspnoea and easy fatigability NYHA functional class 2 since 5 years. She had undergone PTMC in 2007. Upon evaluation, she was found to have severe AS with aortic annulus- $18 \mathrm{~mm}$ and moderate AR with severe MS. Intraoperatively she had severely calcific aortic annulus with calcium extending into interventricular septum. Both the native valves were excised, Manougian's posterior root enlargement and DVR was done with \#25 TTK Chitra mechanical tilting disc valve in aortic position and \# 31 TTK Chitra mechanical tilting disc valve in mitral position with total chordal preservation. Total cross clamp time was 310 minutes and CPB time was 406 minutes. Post CPB TEE showed aortic valve prosthesis well seated, gradient-14/8 mmHg, mitral valve prosthesis well seated, mean gradient$3 \mathrm{mmHg}$, mild biventricular dysfunction. Post-operative period was uneventful and was discharged on POD-8. Echo at the time of discharge showed aortic prosthesis well seated, gradient- $22 / 11 \mathrm{mmHg}$, mitral valve prosthesis well seated with gradient- $9 / 3 \mathrm{mmHg}$, No paravalvular leak. Good biventricular function.

Conclusion:

DVR with root enlargement has a higher morbidity and complications. However, it should be done meticulously to prevent PPM and to improve quality of life.

\section{RARE CASE OF PULMONARY ALVEOLAR MICROLITHIASIS Phani Krishna M K, Dr. Joseph Raj, M.Ch, Ajay \\ Narasimhan, M. Ch \\ Madras Medical College}

Pulmonary Alveolar Microlithiasis (PAM) is a rare autosomal recessive lung disorder identified by intra-alveolar accumulation of minute calculi called microliths. It is thought to be caused by a mutation of SLC34A2, a protein encoding gene. The protein is a $\mathrm{pH}$ sensitive sodium-dependent phosphate transporter. Mutation in the gene can lead to decreased uptake of phosphate from the alveolar spaces and hence leads to calciumphosphate accumulation in the alveoli. The available case reports are few and the data shows no predisposition to any race or country. Incidence is found to be similar in both sexes and higher in the age groups between 20 and 50 years. The term PAM was coined by PUHR in 1930â

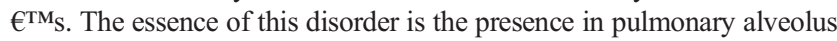
of round shaped little bodies containing concentric calcareous lamellas. Familiality is noted. Chest X Ray, HRCT are diagnostic in most of the cases though biopsy can give confirmatory evidence. The clinical course is not uniform .Lung Transplantation is presently considered an effective therapy though further understanding of the genetics of the disease may allow for a palliative therapy in the future. This case is presented for its rarity and very few case reports having been reported till date.

\section{ANGIOGRAPHIC POOR FLOW AS A RESULT OF INNOMINATE ARTERY STENOSIS IS NOT A CONTRA-INDICATION FOR USAGE OF BILATERAL INTERNAL THORACIC ARTERY \\ Dr. Azhar Sayyed, Dr. Lalit Kapoor, \\ Dr. Pradeep Narayan, Dr. Devraj Kumar, \\ Dr. Sathish Kumar, \\ Rabindranath Tagore international institute \\ of cardiac sciences, Kolkata}

\section{Aims/ Objective:}

Bilateral Internal Thoracic Artery (ITA) grafting is desirable in younger patients. However, assessment of quality of the ITA remains controversial. In presence of Innominate artery stenosis ITA is most often not used by surgeons. However, aim of this report is to illustrate that even in presence of angiographic poor flow BITA can be used for grafting the coronary arteries.

\section{Methods:}

Two patients both females with issues with veins and angiographically demonstrable poor flow in the ITA (Right -1, Left -1) were included. 
Good flow in the other ITA was assessed by angiographic assessment and confirmed by intraoperative free-bleeding technique. In presence of poor flow in RITA, pedicled LITA with skeletonized RITA was used to create LITA-RITA anastomosis in a Y fashion. Similarly In presence of poor flow in LITA a trident anastomosis was created using pedicled RITA and skeletonized LITA. Total arterial revascularization was achieved in both cases. Results:

The procedure was uneventful. Functional assessment for regional wall dysfunction was carried out with trans-esophageal echocardiography. Inotropic requirement was minimal with no post-operative ECG or cardiac enzyme changes. The patients are under regular follow up for 6 and 13 months respectively with no angina or other major adverse cardiac events.

\section{Conclusion:}

Poor angiographic flow of the ITA is not a contraindication for usage of the ITA in presence of other functioning ITA and with careful planning total arterial revascularization is possible if desired.

\section{PRIMARY PULMONARY VALVE REPLACEMENT IN ADULT TETROLOGY OF FALLOT-EARLY RESULTS}

Dr. Sai Surabhi, Dr. Tella Rama Krishna Dev,

Dr. R.V.Kumar

Department of Cardio-Thoracic Surgery,

Nizam's Institute of Medical Sciences,

Punjagutta, Hyderabad.

\begin{abstract}
Aim:
Primary pulmonary valve replacement at the time of total correction for adult TOF helps overcome the problematic complications associated with this spectrum-Thus improving the outcomes with reduced morbidity and no mortality.

Materials and Methods:

This is a prospective study conducted over a period of $2 \mathrm{yrs}$ in adult patients who presented with uncomplicated TOF.

Results:

To avoid post operative difficulties of volume overload, free pulmonary regurgitation and right ventricular dysfunction, we offered primary pulmonary valve replacement as a part of complete correction. There have been 10 patients who under went this procedure along with tetrology repair-standard aortic bi-caval cannulation, cardioplegic arrest, VSD closure. After RVOT resection and pulmonary valve excised, a prosthetic pulmonary valve of appropriate size for the patient is implanted using the continuous suture technique. We choose mechanical valves in males and bio-prosthetic valves in females of child bearing age. There has been no mortality and minimal use of inotropes. Extubation within 4-6 hours, reduced ICU and hospital stay of 5-7 days. An improvement in functional activity and quality of life noted.

Conclusion:

Adult TOF is a burnt out disease with several complications which may not be faced with pediatrictetrology. 1-Multiple collaterals which present a volume burden situation. 2-The pulmonary valve being dysplastic, damaged and hence no more useful in that position. 3-Severe long standing right ventricular hypertrophy more prone to deterioration. To address these 3 main difficulties, we suggest Primary Pulmonary Valve Replacement as a single solution which is simple, safe, reproducible with predictable results and no mortality.
\end{abstract}

\section{TRACHEAL STENOSIS POST CARDIAC PROCEDURES}

Dr. Yousuf Mohammed Rafi, Dr. shivkumar swamy, Dr. Satyaprakash, Dr. Sosle, Dr. Ashit Shetty

Sri Jayadeva Institute of Cardiovascular Sciences and Research

\section{Introduction:}

Tracheal stenosis is a late complication due to cicatrization of tracheal wall after prolonged intubation or tracheostomy. Our initial experience was in patients following OP Poisoning, neurological disorders and injuries. Occurrence of tracheal stenosis following cardiac procedures has significantly increased, due to availability and affordability of cardiac procedures in Tier II and III cities; and early performance of tracheostomies

Aim:

To study tracheal stenosis and its management outcomes in cardiac patients with severe comorbidities and anticoagulation post cardiac procedures

\section{Materials and Methods:}

20 patients who developed symptomatic tracheal stenosis after cardiac procedures, due to prolonged intubation or tracheostomy, from January 2015 to October 2019 were selected. These patients were deemed unsuitable for primary surgical reconstruction due to severity of stenosis (grade III, Grade IV),associated comorbidities, anticoagulation and sepsis All patients underwent T-tube insertion. T-Tubes were positioned under sedation while ventilating through rigid bronchoscope. Flexible videobronchoscopy performed in all patients every 3 months. If effort tolerance was good, and patient asymptomatic, stoma closed permanently.

Results:

13 out of 20 cases $(65 \%)$ occurred post tracheostomy. Mean duration of ventilation was 9 days (Range 7-15). T-tube removed in 10 patients after mean duration of 17 months. T-tube re-inserted in 1 patient within 3 days. 1 patient with Dilated Cardiomyopathy expired during the study. The remaining patients are on regular follow-up. 2 patients had recurrent granulation tissue formation after removal.

Conclusions:

Patients in our study were unsuitable for primary surgical reconstruction in view of Severity of stenosis (Grade III, Grade IV), associated comorbidities, anticoagulation, sepsis. T-tube insertion is a safer alternative palliative as well as therapeutic option in this subset of patients.

\section{MASSIVE PULMONARY EMBOLISM}

AND MASSIVE ILIO-FEMORAL DVT TREATED SIMULTANEOUSLY

Harinder Singh Bedi,Jatin Singh, Vikram Arora

Ivy Hospital, Mohali, Punjab

\section{Objective:}

We present the first documented case of a patient with massive Deep Venous Thrombosis (DVT) and massive Pulmonary Embolism (PE) treated simultaneously

\section{Material and Methods:}

A 46 year old female presented with left leg swelling. She was an operated case of cancer breast on Tamoxifen . On examination she was found to have features of left sided DVT extending up to the iliac vein â $\epsilon^{\text {“ }}$ the IVC was free (Figure 1). She was started on LMWH and DOAC. She developed dyspnea and collapsed. She was successfully resuscitated . A CT angiogram revealed a massive MPA PE (Figure 2). She was referred to our centre in a relatively stable condition on moderate inotropes . She underwent a CDT via the popliteal vein and a CDT of the PE via a catheter from the right femoral vein. Her PA pressure was $\hat{A}^{3 / 4}$ systemic . A multihole Cragg-McNammara catheter was used to deliver the tPA at both sites. Aspiration of clots was also done. The tPA was given over 24 hours .

\section{Results:}

The PA pressure fell immediately. A check venogram revealed a complete resolution of the clot with no iliac vein stenosis.

\section{Conslusion :}

We present a first documented case of simultaneous CDT of a massive PE and a massive ilio-femoral proximal DVT. Isolated congenital pulmonary valve regurgitation (IPVR) is very rare1. Etiologies of absent pulmonary 
valve and bicuspid/dysplastic pulmonary valve are among the more common congenital causes of this condition. Here we present a rare case of IPR caused by absent single pulmonary cusp. A review of literature to establish timing and management of isolated pulmonary regurgitation is also attempted.

\section{NECTAR TO BITTER - INFECTIVE ENDOCARDITIS FOLLOWING PERCUTANEOUS CARDIAC INTERVENTIONS.}

Mohammed Sameer, Vivek V Pillai,

Jaya kumar K, Biju lal S

Sree Chitra Tirunal Institute for Medical

Sciences and Technology

Percutaneous cardiac interventions are on rise and number of cardiac interventions in sick patients with multiple comorbidities have increased. Infective endocarditis (IE) is rare complication of cardiac catheterization and intervention. Though transient bacteremia during cardiac intervention is described, it rarely translates into IE. However, in patients with underlying valvular or congenital heart defects with associated infections and reduced immunity, endocarditis can occur. In this case series, we describe the clinical scenario of endocarditis following invasive cardiac intervention, to improve awareness of this rare clinical problem as well as to re-emphasize the importance of preventive measures. We present course of two patients - one developed native valve endocarditis of aortic valve followed by acute aortic regurgitation who was successfully operated in time and healthy. Other patient developed atypical mycobacterial infection secondary to her BMV procedure for her mitral stenosis and succumbed to death due to failure.

\section{STUDY OF THE OUTCOME OF V-A EXTRACORPOREAL MEMBRANE OXYGENATION ASSISTANCE IN PATIENTS UNDERGOING HIGH-RISK PERCUTANEOUS CORONARY REVASCULARISATION}

Dr Sanjay Kumar, Dr Vladimir Shumaster 1 Department of Cardiothoracic Surgery, Institute of Medical Sciences, Banaras Hindu University, Varanasi, India; 2 Division of Cardiac Surgery, Yale New Haven Medical School, New Haven, Connecticut, USA

\section{Background:}

The haemodynamic instability during Complex high-risk percutaneous coronary intervention (PCI) may lead to increased morbidity and mortality. Pre-emptive use of Veno-arterialextracorporeal membrane oxygenation (VA-ECMO) can provide cardiopulmonary support in high-risk PCI and may lead to improved survival.

Methods:

We performed aretrospective analysis of all patients undergoing high-risk PCI who received peri-procedural VA-ECMO for cardiopulmonary support. Results:

A total of 11patients (90\% male, median age 67 (53-83)years), of whom $60 \%$ had previous coronary artery disease in the form of a coronary artery bypass graft $(36 \%)$ and a PCI (14\%) underwent high-risk PCI and received VA-ECMO support. Cannulation was femoral-femoral in all patients. The main target lesion was a left main coronary artery in $78 \%$, a left anterior descending artery in $14 \%$, aright coronary artery in $7 \%$, and $71 \%$ underwent multi-vessel PCI in addition to main target vessel PCI. The median SYNTAX score was $29.7(10-40.5)$ and in 64\% (9/14) there was a chronic total occlusion. Left ventricular function was mildly impaired in $7 \%(1 / 14)$, moderately impaired in $14 \%(2 / 14)$ and severely impaired in $64 \%(9 / 14)$.
Median ECMO run was 2.57/h (1-4). Survival was 93\% (13/14). One patient died during hospitalisation due to refractory cardiac failure. All other patients survived to discharge. Complications occurred in 20\%(2), with one patient developing a transient ischaemic attack post-ECMOand other one developed athrombus in the femoral vein used for ECMO cannulation. Conclusion:

VA-ECMOin high-risk PCI can be successfully used for cardiopulmonary support in selected patients with a good outcome.

\section{LEFT VENTRICULAR REVERSE REMODELING AND BRAIN \\ NATRIURETIC PEPTIDE \\ LEVELS IN PATIENTS UNDERGOING \\ MITRAL AND AORTIC VALVE REPLACEMENT FOR REGURGITANT LESIONS}

Ananthkumar, Hemachandren Munuswamy, Sreevathsa Prasad, Durga Prasad Rath, Ramsankar Padmanaban, Saichandran BV Jawaharlal Institute of Postgraduate Medical Education and Research, Puducherry

\section{Aims \& Objectives:}

Chronic AR and MR produce a state of LV volume overload resulting in LV remodeling with eccentric hypertrophy. The purpose of this study was to assess the LV reverse remodeling following valve repair/replacement for $\mathrm{AR}$ and MR and to assess the relationship of serum Brain Natriuretic Peptide levels to LV reverse remodeling.

\section{Materials \& Methods}

This was a prospective longitudinal study with a sample size of 50 , which included patients with chronic MR or AR or both, with a dilated LV, who underwent valve repair/replacement in our center from January 2018 to July 2019. Echocardiograms were acquired from these patients preoperatively, at POD7 and at 6 months postoperatively and were analyzed. Serum samples were collected from them preoperatively, at POD7, 3rd month and at $6^{\text {th }}$ month postoperatively and were subjected to ELISA test for measuring serum BNP levels.

Results:

The mean age of the study population was $29+/-13$ years and $60 \%$ were male. 20 patients had severe MR, 7 patients had severe AR and 23 patients had both. RHD was the most common etiology (80\%) in the study population. The mean preoperative LVIDd \&amp; LVIDs were $63.2+/-9 \mathrm{~mm}$ and $46.5+/-8.6 \mathrm{~mm}$ which reduced to $56+/-9.8 \mathrm{~mm}$ and $41.5+/-9.8 \mathrm{~mm}$ at POD7 and further reduced to $49.9+/-8.5 \mathrm{~mm}$ and $35.9+/-8.2 \mathrm{~mm}$ at 6 months postoperatively. A significant improvement in the LVEF was noted from $0.47+$ / -0.1 preoperatively to $0.51+/-0.2$ at 7 th $\mathrm{POD}$ and $0.55+/-0.11$ after 6 months. Serum BNP levels measured preoperatively and postoperatively at 7th POD, 3rd and 6th month showed a significant falling trend from $966.6 \mathrm{pg} / \mathrm{mL}$ to $241.98 \mathrm{pg} / \mathrm{mL}$ suggesting an overall improvement in $\mathrm{LV}$ function and a postoperative marker for LV reverse remodeling.

\section{Conclusion:}

Patients with chronic valvular regurgitant lesions with a dilated LV had a progressive reverse remodeling of $\mathrm{LV}$ as evidenced by significant reduction in the LV dimensions postoperatively and a progressive improvement in the LVEF. Their serum BNP levels were high preoperatively and showed a significant drop postoperatively suggesting an improvement in the LV function and its strong association to LV reverse remodeling.

RAREST OF THE RARE- "EWINGS SARCOMA" OF ANTERIOR MEDIASTINUM Dr Paul M Varghese,Dr Sameer Kumar, Dr Priyanka Nunia, Dr Sarvesh Dubey, Dr Yashvinder Kumar, Dr Gobind G Thakur Army Hospital (R\&R), New Delhi 
History : A 39 yr old male, asymptomatic, incidentally detected to have mediastinal mass. CECT chest showed $8.5 \times 15.8 \times 12.6 \mathrm{~cm}$ large anterior mediastinal mass.

Management: underwent excision of mediastinal mass via sternotomy on $01 / 10 / 19$. HPE (B/5741/20) showed Ewings sarcoma / PNET. Post op management â€" started onVAC : IE regimen on $22 / 11 / 19$ and has received 3 cycles. Currently patient is asymptomatic and healthy.

Conclusion :" Primary mediastinal Ewing's sarcomas and Peripheral primitive neuroendocrinal tumor (ES/PNET) are extremely rare and are aggressive soft tissue neoplasms.* The treatment of ES/PNET involves combined modality therapy.

\section{DESCENDING THORACIC AORTA TO BIFEMORAL BYPASS GRAFTING IN AORTOBIILIAC OCCLUSIVE DISEASE - SINGLE CENTRE EXPERIENCE OF SIX YEARS AT GRANT MEDICAL COLLEGE, MUMBAI,MAHARASHTRA. \\ Dr Suraj Wasudeo Nagre, Dr K N Bhosale, Dr M M Joshi \\ Associate Professor CVTS, Grant \\ Medical College, Mumbai, Maharashtra}

\section{Introduction:}

The aim of study is to describe the clinical symptoms, investigation findings and surgical treatment of aortoiliac occlusive disease where abdominal approach not feasible or not possible for aortobifemoral bypass grafting.

Method:

From May 2013 to May 2019 , ten patients were treated with descending thoracic aorto-bifemoral bypass for aortoiliac occlusive disease at Grant Medical College and J J group of Hospitals, Mumbai (Maharashtra). Total 20 limbs were re-vascularized. Demographic data, co-morbid factors, per operative findings were noted. Indication for surgery is juxtarenal occlusion of the abdominal aorta, in most of the cases. In postoperative period, all patients were evaluated for appearance of distal pulsations, primary patency, warmness of foot, symptom relief, wound infection, healing of the ulcer, and complications. CT angiography was done in follow-up. Maximal follow up of two years and minimum follow up of one month was done after surgery.

\section{Results:}

No mortality. Mean duration of surgery was 2.5-5 $\mathrm{h}$ and mean blood loss was $250 \mathrm{~mL}$. Major morbidity includes one graft occlusion. None of the patients developed proximal propagation of aortic thrombus. Ulcers showed healing. All patients had a good quality of life postoperatively. Postoperative angiography showed good re-vascularization.

\section{Conclusion:}

Majority of our patients are young having juxtarenal aortic occlusion young and hostile abdomen so we decided to use DTA as inflow for primary revascularization to avoid entering abdomen and renal injury. Some authors have recommended supraceliac aorta bypass surgery within the retroperitoneal space for juxtarenal aortic occlusive disease but is technically difficult and associated with morbidity. The major limitation to thoracic aorta bifemoral grafting technique is the morbidity rate associated with thoracotomy in a relatively high-risk vascular surgery population. Also, tunnelling in conventional DTA-to-femoral artery bypass is usually a"blind" procedure. For the cardiothoracic surgeon with experience, the use of descending thoracic aorta could be a very good alternative for infrarenal aortic occlusion or extra-anatomic aortoiliac bypass without any morbidity and mortality.
INCIDENTAL FINDING OF

EMPHYSEMATOUS PYELONEPHRITIS

IN A CASE OF DOUBLE VALVE

REPLACEMENT FOR RHEUMATIC

HEART DISEASE

Ratnamalika Kumar, Anuj Sangal, Rajneesh

Malhotra

Max Superspecialty Hospital

Objectives:

Incidental finding of Emphysematous Pyelonephritis in a case of Double Valve Replacement for Rheumatic Heart Disease.

Methods:

We are presenting a very rare case of 21 years old male who underwent double valve replacement and incidentally got diagnosed with emphysematous pyelonephritis on POD4. Patient also showed two paravalvar leaks across aortic prosthesis on review echo on POD5. A percutaneous nephrostomy was performed and surgical correction was done for PVL.

\section{Results:}

A multidisciplinary approach was taken and patient was successfully discharged and following up in OPD. Nephron sparing regimen was taken and patient escaped nephrectomy and was managed by conservative management and $\mathrm{PCN}$.

Conclusions:

Emphysematous pyelonephritis (EPN) is rare acute severe necrotizing infection of the renal parenchyma and its surrounding tissues that results in the presence of gas in the renal parenchyma, collecting system or perinephric tissue. Emphysematous pyelonephritis requires urgent attention because of the life threatening potential associated with the septic complications.

\section{SINGLE STAGE EXTRA ANATOMIC} BYPASS OF AN ADULT INTERRUPTED AORTIC ARCH WITH AORTIC REGURGITATION : A RARE CASE

Dr Ranajit Beeranna Naik, Dr. Chandra Prakash Srivastava, Dr. Sunil Sharma, Dr. Pradeep Kumar Goyal

Narayana Multispeciality Hospital,

Jaipur-302033, India.

\section{Background :}

Adult patients with interruption of the aorta are an extremely rare occurrence. It is commonly associated with additional cardiovascular anomalies. Surgery is the mainstay in the management of the patients. Interrupted aortic arch and associated cardiac disease is complex situation that requires correction either simultaneously or in stages.

\section{Case summary:}

32 year old male planned for aortic valve replacement, incidentally diagnosed with interrupted aortic arch Type A. He was managed with single stage aortic valve replacement and extra anatomical bypass.

Discussion:

Various management options of adult interrupted aortic arch and associated anamolies is discussed.

USE OF MODIFIED ELOESSER FLAP IN THE MANAGEMENT OF COMPLICATED EMPYEMA THORACIS - STUDY OF 150 CASES IN OUR INSTITUTE GRANT MEDICAL COLLEGE, MUMBAI

Dr Suraj Wasudeo Nagre, Dr Manoj Joshi, Dr K N Bhosale

Associate Professor CVTS Grant Medical

College, Mumbai 


\section{Introduction:}

Empyema is the collection of pus within the pleural cavity. Complicated effusions or empyema which do not respond to medical management or intercostal drainage, require surgical intervention. Decortication, Eloesser flap procedure and thoracoplasty are the commonly employed surgical techniques. When the underlying lungs are too diseased to be satisfactorily decorticated and when the patient is very sick, toxic or has other major co morbid conditions, the Eloesser flap is an invaluable option in the management. This technique was first described by Leo Eloesser in the year 1935 for tuberculous empyema in the pre antibiotic era. (1) Various modification of this technique, also called the window procedure is employed now a days when indicated. We endeavored to study the use of this technique in the management of complicated empyema in a tertiary care centre.

Methodology:

From the database, the details of all the patients who underwent surgery for treatment of empyema were retrieved and studied retrospectively.

Results :

Two hundred and fifty two cases of empyema required surgery over the last 8 years. Of these, 150 patients were subjected to a window procedure. Most patients were referred after due medical management by the pulmonologists and twenty patients presented as complications of previous surgeries. 31 patients were smokers and 19 were diabetic. 9 had bronchopleural fistula. 6 of the cultures and 20 of the biopsies were confirmatory of tuberculosis. The mean hospital stay postoperatively was 6.4 days and the average follow up was for 11 months. Most windows healed by 6 months. Diabetes, debility, malnutrition, drug resistant tuberculosis, immunosupression and the presence of ronchopleural fistulae delayed the healing of the window. Four patients required their window to be refashioned in the process of healing and two patients opted to have elective window closure.

Conclusion:

The modified window procedure is an invaluable option in the management of empyema thoracis when indicated. Patient compliance is good and the recovery time acceptable.

\section{MANAGEMENT OF THE \\ COMPLEX SITUATIONS FOLLOWING THERAPEUTIC INTERVENTION \\ IN PEDIATRIC CONGENITAL \\ CARDIAC PATIENTS; SURGEON'S \\ RESPONSIBILITY AND EXPERIENCE \\ OF MORE THAN A DECADE AS A \\ MEMBER OF THE PEDIATRIC \\ CARDIAC TEAM \\ DR. MOHAMMAD FAIZUL ISLAM, Md \\ Zahidul Islam, Ataul Haque, Abdullah Shahriar, \\ ABM Abdus Salam, Kazi Abul Hasan, \\ Syed Abdul Quader \\ National Institute of Cardiovascular Diseases \\ (NICVD), Dhaka, Bangladesh.}

\section{Background:}

Although surgery and intervention has its own pros and cons in the treatment of congenital cardiac defects. In this study we intensely analysed our institutional experience in surgically handing complex situations occurring during trans catheter device closure of atrial septal defects (ASD), ventricular septal defects (VSD) and patent ductus arteriosus (PDA) in pediatric group of patients.

\section{Methods:}

The study retrospectively reviewed 14 patients (age 2-16 years) who were managed surgically on urgent or emergent basis following embolization of devices used for closure of left to right shunting congenital cardiac defects during the period from January 2006 to July 2019. Congenital cardiac defects were atrial septal defects $(n=6)$, ventricular septal $\operatorname{defects}(\mathrm{n}=1)$ and patent ductus arteriosus $(\mathrm{n}=7)$. Risk factors for device embolization and surgical management techniques for retrieval of embolized devices were discussed.

\section{Results:}

Out of 14 patients 11 patients presented within 24 hours and were managed successfully on urgent basis, removed embolized devices in 10 cases under cardiopulmonary bypass (CPB) and did correction of primary congenital cardiac defects. Also 1 patient required repair of injury to right atrial appendage causing cardiac temponade but did not require ASD device retrieval , 1 patient required repair of damaged tricuspid valve by ASD device. And other 3 patients with PDA device were presented after 1 month. Out of those 3 patients 1 patient was successfully managed by PDA device retrieval from RPA under CPB and PDA ligation. There were 1 hospital death due to rupture of iliac vessels and could not offer surgery because of late referral and 1 died due to rupture of aortic aneurysm following PDA device closure.

Conclusions:

Although therapeutic percutaneous catheter based treatment for congenital cardiac defects is gaining popularity day by day but may require urgent or emergent surgical intervention as the only option in case device is embolized or there is cardiac injury. In this circumstance, to provide an uneventful perioperative course, urgent management strategies should be well planned.

\section{SURGICAL REPAIR OF GIANT LEFT CIRCUMFLEX CORONARY ARTERY ANEURYSM}

Dr. Purvang Shah, Dr. Amit Kumar Tripathy,

Dr. Varun Shetty, Dr. Julius Punnen,

Dr. Devi Prasad Shetty

Narayana Hrudayalaya, Bangalore, India

\section{Aim:}

To report a rare case of Giant Aneurysm of the Left Circumflex coronary artery aneurysm in a young adult and its successful surgical repair. Materials and Methods:

A 22-year-old boy presented to us with Inferior wall myocardial Infarction. On evaluation he was found to have a Giant Left Circumflex Coronary Artery aneurysm measuring 19x23mm with thrombus. His transthoracic echo revealed regional wall motion abnormalities in the circumflex territory with severe left ventricular dysfunction. He was offered surgery. Cardiopulmonary bypass was established using aortic and two stage right atrial cannulation. The heart arrested using cold blood cardioplegia. The heart was positioned to expose the aneurysm. The aneurysm was opened at its most dependent part. The proximal and distal ends were ligated and the aneurysm sac marsupialised. A saphenous vein graft was put to the obtuse marginal arising distal to the aneurysm. He was successfully weaned off bypass, extubated on the same day and discharged uneventfully on the fourth post-operative day.

\section{Results:}

At 1year post-surgery, he was asymptomatic, had an ejection fraction of $40 \%$ with the CT Coronary angiogram showing no residual or recurrent aneurysm and a patent vein graft to the obtuse marginal.

Conclusion:

Coronary artery aneurysms are a rare presentation. They are more commonly seen in the right coronary artery followed by left anterior descending artery. Left circumflex coronary artery aneurysms are extremely rare. Occurrence of such a rare aneurysm at a young age with unknown etiology warranted reporting.

\section{A 6-YEAR SINGLE-CENTRE EXPERIENCE} IN, SUTURELESS AORTIC BIOPROSTHESES Andrew Selvaraj, Amir Sepehripour, Umar Hamid, Shyam Kolvekar, Neil Roberts, Wael Awad, John Yap, Kit Wong, Rakesh Uppal, Kulvinder Lall. Barts Heart Centre, St Bartholomew's Hospital, London, UK 


\section{Aim:}

Analyseour experience and results with the use of sutureless aortic bioprostheses, including their use via hemi-sternotomy and Right Anterior Thoracotomy.

\section{Materials and Methods:}

Weretrospectively analysed records from February 2014 till October 2019. Data collected included, pre-operative demographics, EuroSCORE, operative details, post-operative haemodynamic profiles and major adverse cardiovascular and cerebrovascular events.

\section{Results:}

We performed 219 cases. The mean age was $77.2 \hat{\mathrm{A}} \pm 3.2$, mean logistic EuroSCORE was $10.3 \hat{\mathrm{A}} \pm 2.4$, mean EuroSCORE2 was 4.8Â \pm 3.7 . The LivaNova Perceval prosthesis was used in 196 $(89.5 \%)$ cases and the Edwards Intuity prosthesis used in 23 $(10.5 \%)$ cases. The operations performed were isolated AVR in $123(56.2 \%)$, AVR+CABG in $67(30.6 \%)$ and AVR+MVR+/CABG in $29(13.2 \%)$. AVR was performed via hemi-sternotomy in $22(17.9 \%)$ and right anterior thoracotomy in $26(21.1 \%)$ cases. Mean cardiopulmonary bypass and aortic cross-clamp times were $106.5 \hat{\mathrm{A}} \pm 10.7$ and $68.4 \hat{\mathrm{A}} \pm 9.4$ in the overall cohort and $64.8 \hat{\mathrm{A}} \pm 9.7$ and $42.6 \hat{\mathrm{A}} \pm 7.3$ in the isolated AVR group respectively. There were $8(3.6 \%)$ in-hospital mortalities, $3(1.3 \%)$ cerebrovascular accidents and $7(3.1 \%)$ permanent pacemaker insertions. Postoperative valve gradients were $9.63 \hat{\mathrm{A}} \pm 4.2 \mathrm{mmHg}$ (mean) and $16.4 \hat{\mathrm{A}} \pm 7.4 \mathrm{mmHg}$ (peak), with an incremental reduction with increasing prosthesis size. There were $6(2.7 \%)$ mild and $9(4.1 \%)$ trivial paravalvular leaks on post-operative echocardiography. The mean length of hospital stay was $6.2 \hat{\mathrm{A}} \pm 3.2$ days. These outcomes were comparable or superior to an equivalent cohort undergoing conventional sutured AVR.

\section{Conclusions:}

Our results demonstrate the excellent safety and haemodynamic performance of sutureless bioprostheses in this patient cohort as well as their use in the development of a minimally-invasive program.

\section{AN UNUSUAL CASE OF ANTERIOR MEDIASTINAL MASS \\ Dr. N N L Rajitha, \\ Dr. K. Venkata Vijay \\ Katuri Medical College \\ and Hospital}

A 55 year old male teacher by occupation came with complaints of SOB, chest pain and cough for 6 months. He had similar episodes of SOB, cough 6 months back, investigated and diagnosed as right sided pleural effusion and ICD insertion was done and started on ATT. As symptoms are not relieved, he was again investigated and found to have left sided pleural effusion and ICD insertion was done and was on ATT. He was referred to our hospital for recurrence of disease. At our hospital he was evaluated and found to have a large mediastinal cyst with bilateral intra thoracic extension.on examination he was found to have features of morquio syndrome with kyphoscoliosis and dwarfism. He had pectus carinatum and bilateral decreased breath sound in infraclavicular and mammary region. $\mathrm{Ct}$ chest showed a large lobulated cystic lesion in anterior mediastinum partially encircling the vascular structures and cardia with no enhancing internal septations or solid areas. Excision of cyst was planned under General anaesthesia. Intraoperatively it is a dumbbell shaped cyst of $15 \tilde{\mathrm{A}}-20 \mathrm{cms}$ abutting anterior pericardium and cyst was extending into mediastinal pleural and both sides of chest. Excision was done and sent for histopathological examination and was confirmed as bronchogenic cyst
ANATOMIC CORRECTION OF ALCAPA IN AN ADULT PATIENT

Dr Nikunj R Shekhada,

Dr B R Jagannath

Star Hospitals, Banjara hills, Hyderabad

\section{Introduction:}

Anomalous origin of the left coronary artery from the pulmonary artery (ALCAPA) is a rare congenital anomaly with an incidence of 1 in 300,000 live births and represents 0.25 to 0.5 per cent of all cases of congenital heart disease. Most of the cases $(85 \%)$ are diagnosed within first month of life. Nearly 90 per cent of untreated patients die within one year with myocardial ischemia and infarction or congestive cardiac failure with mitral regurgitation. However, a few patients can survive into adulthood due to adequate collateral blood supply to the left coronary circulation through dominant right coronary artery. Regional wall motion abnormality is commonly seen in most of the patients with ALCAPA surviving till adult life. Adult ALCAPA can present as effort angina due to relative ischemia and coronary steal. Acute presentation though common in infancy, is relatively rare in patients who survive to adulthood due to adequate collaterals.

\section{Background:}

Here we present a 37 year Female patient presenting as exertional dyspneaand being finally diagnosed as adult ALCAPA in the cath lab. The patient was successfully treated with

\section{Conclusion:}

ALCAPA can present in adults and can have variable expression ranging from asymptomatic LV dysfunction, effort angina, and acute coronary syndrome to refractory arrhythmia or even sudden cardiac death. Though it is a congenital coronary anomaly it should still be kept in mind while dealing with an adult patient with such type of presentation. Echocardiography can sometimes miss the diagnosis, especially in adult patients, even if specially looked for.

\section{XENOGRAFT RECONSTRUCTION OF RVOT IN TRUNCUS ARTERIOSUS \\ Dr. Aryakamal Das, DR. Debasis \\ Das, DR. Nilanjan Dutta, \\ Dr. Shubhadeep Das, Dr. Amitava \\ Chattopadhyay, Dr. Rangan \\ Koley, Dr. Manish Sharma \\ Narayana Superspeciality \\ Hospital, Howrah, West Bengal}

\section{Objectives :}

To report the clinical outcomes of children who underwent xenograft reconstruction of RVOT for Truncus Arteriosus in our institute.

\section{Methods}

We retrospectively collected data and studied 7 patients who underwent xenograft reconstruction of RVOT for Truncus Arteriosus between May 2018 and November 2019 in our institute. All clinical parameters including mortality and morbidity were analyzed.

Results :

7 patients underwent surgery for Truncus Arteriosus in our institute with a mean age of 38.1 months, mean weight of $10.6 \mathrm{Kgs}$. Out of 7 patients, 4 were of type $1 / 2,1$ patient was of Type 1 and 2 patients were of Type 2 . The RVOT was reconstructed with Bovine Jugular vein xenograft (ContegraTM). 3 patients received conduit of $12 \mathrm{~mm}$ and 3 patients received conduit of $14 \mathrm{~mm}$ and 1 patient received conduit of $18 \mathrm{~mm}$ (mean $13.7 \mathrm{~mm}$ ). Mean cardiopulmonary bypass time was 168.71 minutes, with a mean cross clamp time of 117.42 minutes. 4 out of 7 patients had significant Truncal valve regurgitation, which was repaired. There was one mortality on the 36 th post-operative day due to sepsis. Out of the survivors, the mean postoperative stay was 6.5 days. 


\section{Conclusion :}

Xenograft reconstruction is a safe and readily available effective option for RVOT reconstruction in Truncus Arteriosus. It provides a competent RVOT in a severe PAH. The pitfall is future need of conduit revision.

\section{STERNAL WIRE SITE INFECTION IN A PATIENT WITH LVAD HEART MATE-III}

Dr. N. Madhu Sankar, Dr. Anto Sahayaraj R, Dr. Aarimuthuswamy A, Dr. Kirthivasan V. Fortis Malar Hospital, Chennai

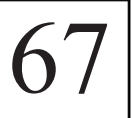

Sternal wire site infection in a patient with LVAD Heart MateIIIInfection along with GI bleeding, stroke are the leading cause of unplanned readmission after LVAD insertion. Driveline infections are the most common type of LVAD- associated infection (LVADI). However infection can occur at surgical incision site including sternotomy/Thoracotomy incision. This report describes a case of sternal wire site infection- 3 years post LVAD insertion. Case report51year old male, from Kazakhstan a known case of ischemic cardiomyopathy had undergone CABG. Subsequently he had stenting of native coronaries as well as vein graft stenting. His LV function deteriorated and underwent insertion of Heart Mate-III through midline sternotomy, in Kazakhstan. He was doing well and was on regular follow up. He presented to us with a small sinus in lower one third of sternal incision. CT scan revealed localized sternal wire infection and was confirmed with PET scan. Sinogram was done to rule out proximity to drive line as well as assist device. He underwent successful sternal wire removal and listed for heart transplant. Continuous vigilance and regular follow-up is mandatory to improve the outcome patients with Left ventricular assist devices.

\section{COR TRIATRIATUM IN}

\section{ADULT - CASE REPORT}

Pratik Pandey, Lalit Kapoor,

Dhiraj Barman

Rabindranath Tagore International Institute

of cardiac sciences Kolkata

\section{Background:}

Cor Triatriatum is a rare congenital disorder, where left atrium is divided into two chambers by a fibromuscular membrane. This results in a double chambered left atrium which effectively creates three Atrial chambers (including the right) giving the anomaly its name.

Aims/ Objectives:

To report a case of Presentation of Cor Triatriatum as new onset Atrial fibrillation in an adult patient which is an uncommon finding.

\section{Material and Method:}

We report a case of Cor Triatriatum in adult patient with symptoms and surgical treatment through left atrial approach.

Results:

We operated on this patient through left atrial approach. Intraoperative and Postoperative period was uneventful. On followup patient is doing well.

\section{Conclusions:}

Presentation of Cor Triatriatum as new onset Atrial fibrillation in an adult patient is an uncommon finding. Additional procedures for correction of arrhythmia are usually not required in young adults. Corrective surgery is required to provide the relief of symptoms in majority of cases. Life expectation after repair of classic Cor Triatriatum approaches that of general population.
INTRACTABLE HEADACHE IN

PATIENT WITH SINGLE LUNG

\section{TRANSPLANT}

Dr. N. Madhu Sankar, Dr. Aarimuthuswamy,

Dr. Jackin Moses, Mrs. Shabana Shabanam,

Dr. K.M. Cherian

Frontier Lifeline Hospital, Chennai

Tacrolimus induced neurotoxicity has been well documented in solid organ transplant recipients. The major neurologic side effects occurs in 5 to $8 \%$ of transplant recipients.

\section{Case report:}

A 61 year old lady from Kuwait has undergone Single lung transplant (right) in 2016 for end stage interstitial lung disease, Idiopathic pulmonary fibrosis, Moderate PAH, Suspected Sjogren's syndrome. She was on triple drug immunosuppressive therapy with tacrolimus, mycofenolate and low dose of steroids. She was on regular follow up and doing well. Recently she presented with severe headache with mild tremor. MRI Brain was done which showed subtle ill- defined restricting T2/T2 FLAIR hyper intense signal in the parietal white matter bilaterally- suggestive of early leukoencephalopathicchanges attributed to tacrolimus neurotoxicity. Her Tacrolimus dose was optimized. Neurologist opinion was obtained. She was advised Trodat Scan Brain, Pet Scan Brain, NCS / Nerve Conduction Study. With modification of Tacrolimus doses and addition of neurtrophic drugs he showed substantial clinical improvement.

\section{PREOPERATIVE LEVOSIMENDAN HAS BENEFICIAL EFFECT ON RIGHT VENTRICULAR FUNCTION IN PATIENTS WITH SEVERE LEFT VENTRICULAR DYSFUNCTION UNDERGOING CORONARY ARTERY BYPASS GRAFTING \\ Dr. Dhanesh Kumar, Dr. Op Yadava, \\ Dr Vikas Ahlawat, Dr Anirban \\ Kundu, Dr. Amita Yadav, Dr. Rekha \\ Mishra, Dr. Arvind Prakash, \\ Dr. Vinod Sharma \\ National Heart Institute, New Delhi-110065}

Levosimendan is an effective agent of the class of Calcium sensitizers, having key complementary mechanisms of action: Calcium sensitization; opening of ATP dependent $\mathrm{K}+$ channels, both on the sarcolemma of the smooth muscle cells in the vasculature and in the mitochondria of cardiomyocytes. Levosimendan has been recognized to have a long acting metabolite with a halflife of about $80 \mathrm{hrs}$. There have been few small studies of this drug on right ventricular (RV) function worldwide. Keeping this in mind we studied the effect of Levosimendan on RV function in CAD patients. This was a prospective, randomized, double blind study of 50 patients of CAD with severe left ventricular dysfunction (LVEF-35\%) undergoing elective off pump CABG. We found Levosimendan has inotropic effect on RV myocardium and vasodilatory effect on blood vessels. It causes significant decline in PVR (p\&lt;0.018), RVSP (p\&lt;0.001) and PASP (p\&lt;0.001). It also improves diastolic function of RV as shown by fall in RV Tei index (p\&lt;0.001), decrease in RV end-diastolic pressures and $\mathrm{E} / \mathrm{E}$ tricuspid annulus (p\&lt;0.006). However, we did not find beneficial effects on ICU, hospital stay $(\mathrm{p}=0.164$ \&amp; $p=0.349$ respectively) and mortality benefits in our study between two groups. Based on the findings of our study, we advocate that Levosimendan has salutary effects on RV function in patients with severe left ventricular dysfunction undergoing CABG in form of improved PASP, RVSP, E/E, RV Tei index and PVR. 
STUDY OF DEMOGRAPHIC

PROFILE, CLINICAL FEATURES,

DIAGNOSTIC MODALITIES, AND

HISTOPATHOLOGICAL FEATURES

OF MEDIASTINAL TUMOURS

S.G.K Arif, R. Basu

R.G.Kar Medical College \& Hospital. Kolkata.

\section{Objectives:}

1) To study the Demographic profile, Clinical spectrum \&amp; modes of presentation of various mediastinal masses2) To know our institutional based incidence of individual (Histopathological) mediastinal masses. 3) To study the histopathological variance and outcomes of various mediastinal masses 4) To study the various treatment modalities with special emphasis on surgical intervention and its variables

\section{Study design:}

A prospective, observational study Data collected from amixed population,various parts of West Bengal STUDY POPULATIONPatients with mediastinal tumours presenting or referred CTVS.R.G.K.M.C.HTIME FRAME - conducted from June 2015 to June 2019SAMPLE SIZE-68MethodologyPresenting symptoms, physical findings, and other demographic information were recorded for each patient as an case sheet. Using preoperative radiographic studies and intraoperative findings, the size of the tumors and their relations to vital structures were observed. The postoperative hospital stay, chest tube duration, and change of patient symptoms were recorded. Longer term outcomes included the patientsâ $€^{\mathrm{TM}}$ activity levels at the 4week,3mnths, 6 mnths postoperative clinic visit, resolution of symptoms, evidence of cyst or neoplastic recurrence, and other late complications Result:

The ages of the 68 patients ranged between 7 and 79 years, (mean $\hat{A} \pm$ s.d was $46.09 \hat{\mathrm{A}} \pm 17.55$ years). Most of the patients $(58.7 \%)$ were in the age group between 40 â " $^{\prime} 69$ years .There were 49 male patients whose ages ranged between 7 and 79 years(mean $48.79 \hat{\mathrm{A}} \pm 17.05)$ and 19 female patients whose ages ranged between 15 and 74years (mean $41.31 \hat{\mathrm{A}}$ \pm 18.13 ). Symptoms were present in 57 patients $(83.8 \%)$ out of the 68 patients and 11 patients $(18 \%)$ were asymptomatic. 17 of the symptomatic patients $(29.8 \%)$ had malignant disease while 40 of the symptomatic patients $(70.17 \%)$ had benign disease. There were 24 patients $(35.3 \%)$ with malignant mediastinal masses and 44 patients(64.7\%) with benign masses. The incidence of symptoms among patients with malignant mediastinal masses was $85 \%$.. The incidence of symptoms among the patients with benign disease was $80.5 \% 66.2 \%$ cases in our series had history of tobacco use which was significantly higher. The commonest symptoms in this series were cough, dyspnea and chest pain. These symptoms were more frequently associated with benign disease. The anatomical location of the tumours in our series was anterior mediastinal $(45.6 \%)$ superiormediastinal $(20.6 \%)$ anterosuperior $(1.5 \%)$, middle mediastinal $(13.5 \%)$, posteriormediastinal $(17.6 \%)$, multiple $(1.5 \%)$.In our Series Out of 66 patients 53 patients $(81.3 \%)$ underwent open resectionwhilst $13(19.7 \%$ ) underwent video assisted Minimal access procedures. Posterio-lateral thoracotomy approach was the most common approach with 16pts (24.2\%) undergoing it. Anterio-lateral thoracotomy was used in 15 pts $(22.7 \%)$ followed by lateral thoracotomy in $19.7 \%$ and sternotomy in $13.6 \%$ Mediastinal mass excision was performed in 24 patients $36.3 \%$.Excision Biopsy was the second most commonly performed surgical procedure 13 cases $(19 \%)$ in our series. 7 out of the 13 VATS cases comprised of tubercular lymph node masses. The Most common tumor/Mediastinal masses were thymoma comprising of 15 outof 68 cases $(22 \%)$. The second most common masses encountered were tubercular lymph node masses (10.2\%).Lymphoma (NH ) constituted the 3rd most common tumour $8.8 \%$ in our study followed by thymic hyperplasia (7.3\%).Poorly differentiated carcinoma constituted the second most common malignant tumour inthe mediastinum(7.3\%) following lymphoma. Thymoma was the most commonanterior mediastinal tumour in our study. Lymphoma and Bronchogenic cyst (5.8\%)and dermoid cyst $(4.4 \%)$ were common in middle mediastinum .Neural tumours like neurofibroma and ganglioneuroma were the predominant tumours in posteriormediastinum. Most tumours extended into the Right hemithorax and thus right lateralthoracotomy was used in $62.1 \%$ cases. Left lateral thoracotomy was used in $22.7 \%$ and supine decubitus was used in $15.2 \%$ cases. The mean duration of operative surgery was $100.45+/-46.63 \mathrm{mi}-$ nutes. The mean blood loss was $230.30+/-193.13 \mathrm{ml}$.Major blood $\operatorname{loss}(\& \mathrm{gt} ; 500 \mathrm{ml})$ was found in 6 cases $(9.1 \%)$ and wasthe cause of mortality in one case. Major complication were seen in 23 cases out of66 cases. Most common intra-op complication was presence of multiple adhesions and loculations (34.7\%) followed by bleeding (26\%).Mean duration of post opventilation was $15.45+/-23.7 \mathrm{hrs}$ with 48 cases $86.4 \%$ cases were extubated within $24 \mathrm{hrs} .11$ cases did not require any post op ventilation. Mean duration of ICU stay was 7.09+/-3.32 days.60 patients (90\%)were shifted out of ICU within14 days. Mean duration of hospital stay was $14.53 \hat{\mathrm{A}} \pm 6.70 .59$ patients $(89.48 \%)$ were discharged alive within 3 weeks. Post operative complications were seen in 32 out of 66 cases $(48.4 \%)$ most common post op complication was excessive drain output5 cases (15.6\%).Infection and fever constituted $15.6 \%$ and $12.5 \%$ respectively. The overall mortality with treatment was $10 \%$ i.e 7 out of 68 patients. 4 patients died as aimmediately post surgical intervention while 3 patients died during adjuvant therapy

\section{Conclusion:}

1) There is no age sex predilection for mediastinal tumours in general2) Thymoma was the most common anterior mediastinal tumour in ourstudy. Lymphoma was common in middle mediastinum .Neural tumours were the predominant tumours in posterior mediastinum.3) Thymic tumours were the most common tumours of mediastinum followed by tubercular masses4) CT scan was the gold standard investigation for diagnosis and management of Mediastinal masses5) Surgical resection should be offered to all symptomatic mediastinal masses unless contraindicated6) Mediastinal masses have 10\% mortality with treatment

\section{OUR EXPERIENCE WITH ISOLATED SUPERIOR MESENTERIC ARTERY BYPASS FOR INTESTINAL ISCHEMIA; IS IT GOOD ENOUGH?}

Kuntal Surana, Chaitanya Raut, Prashant Mishra, Vaibhav Shah, Manish Jadhao, J. V. Khandekar

Lokmanya Tilak Municipal

Medical College and General

Hospital

\section{Objective:}

A number of reports indicate revascularization for intestinal ischemia should include the superior mesenteric artery (SMA) and the celiac artery. However, no controlled or randomized studies have proven this approach superior to SMA bypass alone. We report our results using bypass to only the SMA for intestinal ischemia based on the observation that postprandial intestinal hyperemia is limited to the superior mesenteric artery (SMA).

Design:

Retrospective observational review with short term follow up of up to 12 months.

\section{Patients/Methods:}

The records of patients who underwent intestinal revascularization of the SMA alone from 2011 through 2018 were reviewed. Patients were assessed for indication for operation, operative technique, perioperative mortality, and short-term outcome. The SMA grafts were examined for patency within 6 months post operatively using duplex scanning or arteriography.

Results:

Eighteen bypasses to only the SMA were performed in 18 patients (10 female and 8 male; mean age, 59 years; age range, 13 to 81 years). 
Indication for operation was symptomatic chronic mesenteric ischemia in 15 cases and acute intestinal ischemia in 3 cases. There were no perioperative deaths. Graft patency and patient survival rates were $89 \%$ and $100 \%$ respectively on short term follow up.

\section{Conclusion:}

Revascularization of only the SMA for intestinal ischemia provides excellent graft patency with acceptable perioperative mortality and Shortterm patient survival. The SMA bypass alone for intestinal ischemia appears as successful as bypasses to multiple visceral vessels at least on short term follow up.

\section{PER OPERATIVE GRAFT FLOW MEASUREMENT WITH TRANSIT \\ TIME FLOWMETRY AND INDICATIONS FOR GRAFT REVISION OUR EXPERIENCE}

Vikram Pal Singh, Sameer kapur, Arun garg, Gurmeet Singh, Rajiv Kumar, Sarju Ralhan, Bishav Mohan, GS Wander

Dayanand Medical College and Hospital Ludhiana, Punjab

Early graft patency is a major determinant of morbidity and mortality following coronary artery bypass surgery. Long-term graft failure is caused by intimal hyperplasia and atherosclerosis, while early failure, especially in the first year, has been attributed, in part, to surgical error. The need for intraoperative graft evaluation is paramount to determine need for revision and ensure future functioning grafts. Traditionally in patients undergoing coronary artery bypass grafting, parameters for graft revision include haemodynamic instability, electrocardiographic changes of myocardial infarction, new regional wall motion on transesophageal echocardiography abnormalities. But graft failure occurs even in absence of these signs. Various techniques are available to evaluate graft patency include Transit time flow metry (TTFM), fluorescence dye imaging and epicardial ultrasound (ECUS). There is paucity of data of their use in India. TTFM is in use since 1990 and is useful adjunct for quality assuranc for CABG. In our centre we are doing anaortic arterial revascularisation using bilateral internal thoracic arteries since 2015 exclusively. We measured grafts flow using Mira Q (Medistim, Norway) transit time flow meter in 250 patients per operatively. We follow strict protocol of checking the flow in the both arteries used in various configurations before protamine and if the measurements do not meet the criteria, we revise the graft. In this presentation we share our experience.

\section{MEDIASTINAL DERMOID CYST WITH RUPTURE IN THE PERICARDIUM: A RARE CASE REPORT}

Dr Chaitanya Raut,Dr Prashant Mishra

Lokmanya Tilak Municipal General Hospital,

Sion, Mumbai.

\section{Introduction:}

Mature cystic teratoma (dermoid cyst) is the rare neoplasm arising from all three embryonic germinal layers. Mediastinum is the second most common site for teratomas. We present a rare case of mature cystic teratoma with rupture in the pericardium.

Case report:

A 12-year-old female presented with low grade fever and retrosternal chest pain for 7 days. On investigations (CECTchest) she was found to have a large cystic lesion in the mediastinum with pericardial effusion. During surgery a $9.5 \mathrm{~cm}$ x $9 \mathrm{~cm} \times 5 \mathrm{~cm}$ sized infected cyst was seen arising from the right lobe of thymus with rupture in the pericardial cavity. Complete excision of the cyst was done along with right lobe of thymus and part of adjoining pericardium. the cyst was confirmed to be dermoid cyst on gross and histopathological examination.

Conclusion:

Teratoma is a rare neoplasm in the mediastinum with rupture in the pericardium even rarer. This should be suspected in a young patient with nonspecific chest complaints and mediastinal widening on $\mathrm{X}$ ray. $\mathrm{CT}$ chest is usually diagnostic of dermoid cyst and tissue biopsy is rarely required. Rupture into the pleura and pericardium can occur and should prompt early surgery. Complete excision of the tumour is the definitive treatment

\section{REPORT OF A CASE OF SACCULAR ANEURYSM ARISING FROM AORTIC SINUS AS A COMPLICATION PHV ENDOCARDITIS}

Sujoy Chatterjee, Srirup Chatterjee, Rajarshi Ghosh, Santosh Kumar Pandey, Lakshmi Sankhayan, Nikhil

Bansal, Satyajit Bose

The Mission Hospital, Durgapur

\section{Introduction:}

Infective endocarditis is a serious complication of PHV and leads to mayrid of structural abnormalitis of heart. We are presenting a case of saccular aneurysm arising from aortic sinus as a consequence of PHV endocarditis. Case Report:

29 year old lady who underwent AVR with tissue valve for Rheumatic Aortic Valve leison 5 years back presented with low grade fever on and off for last 1 month. Blood investigations revealed increased TLC count (\&gt;12,000) and CRP levels. Echocardiography revealed severe PHV degeneration with a mean gradient \&gt; $50 \mathrm{~mm}$ of hg, a vegetation on the cusps corresponding to NCC and hugely dilated sinus greater than $6.5 \mathrm{~cm}$. She also had severe low pressure TR. Blood cultures were negative most likely due to multiple courses of antibiotics due to fever over last 1 month. CT angiography further clarified the findings. A scauular aneurysm arising from sinus between LCC and NCC, the mouth of which was just laterql to left coronary ostia. It measured 7.1 at maximum diameter and occupied a position between aorta, SVC and atria. Ostia of Right coronary artery was occuled by debris. She was operated for Bentall with SJM Valved conduit with TV repair with $30 \mathrm{~mm}$ ring annuloplasty. Post operative period was uneventful, extubated on POD 1 morning. All tissues have been sent for both bacterial, fungal and anaerobic cultures. Discussion Saccular aneurysm of Sinus of Valsalva as a complication of PHV endocarditis is a rare complication. It can lead to serious life threatening haemorrhage and immediate death if not detected early and treated.

SUBMITRAL ANEURYSM OF

\section{THE LEFT VENTRICLE.}

Dr Tejas Dandekar, Dr Manish Jadhao,

Dr Arnab De, Dr Noaman Shaikh

LTMGH SION

\section{Introduction:}

Submitral aneurysm is a rare cardiac pathology of obscure origin and varied clinical manifestations with symptoms being produced by mitral regurgitation, thromboembolism, arrhythmias, diastolic dysfunction, and coronary compression.

\section{Aim and method:}

We retrospectively analysed 6 patients with sub-mitral aneurysm which were managed surgically at our institution between 2014 and 2018. The sub-valvular aneurysms were diagnosed pre operatively by means of transthoracic echocardiography and cardiac CT. An extra cardiac approach was used in the surgical management of all the patients. Results were analysed in terms of immediate post-op survival, need for re-surgery and 6 month survival on follow-up.

Results:

Six patients were operated for sub mitral aneurysm( 4 females, 2 males with mean age of $51.4+/$ - 4years) at our institution. None of the patients 
underwent mitral valve replacement/repair. Two patients were subjected to repeat surgery. One in view of failure of closure of aneurysm and another one in view of bleeding. The patient with failure of closure of aneurysm succumbed in the immediate post-op period. 5 patients on their 6 month follow-up showed symptomatic improvement, with only 1 patient having persistent moderate mitral regurgitation.

Conclusion:

Surgical management is the mainstay therapy for patients diagnosed with sub-mitral aneurysm. Extra cardiac approach is easy and safe with good surgical outcomes.

\section{CAROTID ARTERY PSEUDOANEURYSM}

Ajil Joseph, S. Mahapatra

Institute of Post Graduation

Medical Education \& Research

SSKM Hospital

\section{Introduction:}

Carotid artery puncture is a common complication of internal jugular vein cannulation. Here we report a fatal complication of a pseudoaneurysm after an attempted internal jugular vein cannulation.

Case report:

A 55yrs old patient presented with large firm swelling on right side of neck since 8 mnths following IJV cannulation for dialysis. It was non pulsatile, measuring $12 * 10 \mathrm{cms}$. Ultrasound neck and CT angiogram revealed it to be carotid artery pseudoaneurysm. Patient was operated and pseudoaneurysmal sac excised. Small rent was seen in common carotid artery which was closed with prolene 6-0. Flow through carotid artery was immediately restored.

\section{Discussion:}

Iatrogenic pseudoaneurysm occur due to placement of hemodialysis catheter into carotid artery. Inadvertent arterial puncture occur in 5\% of central venous catheterisation but development of pseudoaneurysm is rare. Complication include hemorrhage, cerebrovascular accident due to hematoma, airway compression, hemothorax and death. Unrecognized intra arterial placement of catheter is relatively uncommon. Pulsatile flow and bright red colour of arterial blood need not be present always, such as in hypovolemic and hypotensive patients. if there is uncertainty about the location, a pressure transducer may be connected to the scouting needle, and the differences in arterial and venous waveforms may be identified. Peudoaneurysm develops if hemorrhage is not controlled immediately and the rent repaired.

\section{Conclusion:}

Intravenous catheterization is a common procedure, but if not done carefully can lead to fatal complication such as arterial pseudoaneurysm, which can be avoided with right technique and immediate repair.

\section{IMPACTED DISC BATTERY CAUSING ACQUIRED TRACHEOESOPHAGEAL FISTULA IN A CHILD.}

Dr David Idowu, Prof.

Rajan Santosham,

Dr Rajan Santosham,

DR Laksmi P. Naga Sai

Santosham Chest Hospital,

155 Egmore High Road, Egmore,

Chennai

\section{Background:}

The ingestion of disc battery and its consequent aerodigestive complication is less publicized, and its danger is less understood by both parents and caregivers. The management of children with disc battery ingestion is a costly affair associated with unpredictability and high morbidity. There is a need to educate the parents and update health-care givers on the trend of care.

\section{Case Presentation:}

We present a case of 18-month-old male who ingested a disc battery that became impacted in the upper esophagus, it was removed about 4 hours later. Follow-up endoscopic and radiological evaluations later diagnosed with tracheoesophageal fistula. After careful multidisciplinary preoperative planning, he had surgical repair via anterior cervical approach with a good outcome.

Conclusion:

Delayed two hours) removal of impacted disc battery from the esophagus can result in TEF. The complication is common with children less than 4 years. Removal of this foreign body should be followed carefully for the diagnosis and treatment of these fistulae.

\section{ROLE OF INTRA- AORTIC BALLOON PUMP IN INTRACTABLE CARDIOGENIC SHOCK FOLLOWING VALVULAR SURGERY}

Dr Jigar Shah, Dr Vivek Wadhawa,

Dr Chirag Doshi , Dr Divyesh Rathod

$\mathrm{U} N$ mehta Institute of cardiology and research center

\section{Background :}

Mechanical circulatory support may be necessary to prevent irreversible end-organ damage in cardiogenic shock. Post valvular surgery incidence of severe LV dysfunction is around $8.6-11 \%$. Our aim is to evaluate the role of intra-aortic balloon pump (IABP) in intractable cardiogenic shock following valvular surgery, assess prognostic factors and patient survival. Patient and Methods :

A single center retrospective study was performed in 19 (3.37\%) patients with cardiogenic shock following valvular surgery supported firstly by IABP in the year 2017.

\section{Results:}

Out of all valvular surgery patients (N-567), 64 patients developed severe LV dysfunction. Amongst them 19 patients underwent IABP insertion. In all patients after putting IABP at 24 hour, lactate decreased from 3.7 (2.8-7.4) to $1.6(1.4 \hat{a} €$ " 2.5$) \mathrm{mmol} / \mathrm{L}(\mathrm{p}=0.001)$. Twelve patients $(63.15 \%)$ defined as IABP responders were successfully weaned from IABP in 2-3 days with ionotropic support and discharged to home. IABP failed in seven patients (non-responders, 36.85\%) who either died $(\mathrm{n}=4)$ or needed support by extracorporeal membrane oxygenation $(\mathrm{n}=3)$. At 24 hour of IABP support, urinary output was higher $(2,890[1,73-4,625]$ vs. $1,150[680-2,125] \mathrm{mL} ; \mathrm{p}=0.001)$ and fluid balance more negative $(1,450[2,572$ to 1,134$]$ vs. 500 [965 to $+255] \mathrm{mL} ; \mathrm{p}=0.001$ ) in responders than non-responders. Overall survival at 1 year was $67 \%$.

\section{Conclusion:}

In most patients, first-line support by IABP is safe and cost effective in intractable cardiogenic shock following valvular surgery is associated with improvement in organ perfusion and clinical stabilisation for at least 24-48 hours allowing time for decision making on next therapies.

\section{VACUUM ASSISTED CLOSURE- FOR TREATMENT OF DEEP STERNAL \\ WOUND INFECTIONS- OUR EXPERIENCE OVER 3 YEARS}

Dr. Gaur Hari Das,

R.G. Kar Medical College and Hospital, Kolkata

\section{Background:}

The VAC system (vacuum-assisted wound closure) is a non-invasive negative pressure wound therapy (NPWT) for wounds. In this paper we report our experience of management of postoperative deep sternal wound infection by debridement followed by VAC application. 
Aims and objective :

1.To study etiology of DSWI in our setup 2.To study incidence of various risk factors 3 . To know outcome after VAC therapy for sternal wound infection Material and Method:

Retrospective study of 17 patients ( 10 men, 7 women) out of 480 patients (operated for various cardiac surgery) with a median age of $66.5 y$ years (range 48 to 75 years) with sternal wound infection at CTVS department of R. G. Kar Medical College, Kolkata, $(\mathrm{CABG}=9, \mathrm{MVR}$ post $\mathrm{CMC}=3$, $\mathrm{DVR}=3, \mathrm{AVR}=1, \mathrm{MVR}=1$ ) were approached with the VAC system after initial surgical debridement.

Result:

Complete healing was achieved in 15 patients. The VAC system was removed after a mean of 12.3 days (range 7 to 20 days). In 15 patients $(88.2 \%)$, the VAC system followed by primary closure of sternum. In 1 patient $(5.8 \%)$ VAC was used as a bridge toreconstructive surgery with a pectoralismuscle flap and in 1 patient $(5.8 \%)$ VAC followed by omental flap coverage of sternal wound. Intensive care unit 4 to 10 days (median 6 days). Duration of hospital stay 15 to 45 days (median 30 days). Inhospital mortality was $0 \%$, and 30-day survival was $100 \%$ directly with use of VAC. The median duration of the vacuum-assisted closure therapy was 13 days (range 7 to 20 ).

\section{Conclusion:}

The VAC system accompanied with debridement can be considered as an effective and safe adjunct to conventional treatment modalities for sternal wound infections after cardiac surgery. In our opinion vacuum-assisted closure therapy offers a safe, reproducible option for postoperative deep sternal wound infection to sternal stabilization and complete healing.

\section{INCIDENCE AND IMPLICATIONS OF CORONARY ARTERY DISEASE IN ROUTINE VALVE SURGERY ABOVE THE OF 40 YEARS \\ Kikesh C Patel \\ KEM hospital}

\section{Aims \& objectives:}

We evaluated incidence \&amp; implication of Coronary artery disease in patients above 40 years presenting for valve surgery.

Material \& methods :

Between October 2017 \&amp; October 2019 Coronary Angiogram was performed in all such patients. SAMPLE SIZE:231 patients TYPE OF STUDY: Prospective observational study

\section{Results:}

Out of 231 patients, Coronaries were normal in 208 patients (Group I) and diseased in 23 (Group II). Valve replacement along with CABG was done in all 23 patients. Out of 23 AVR was done in 13, MVR In 8 and DVR in 2. Hypertension and diabetes more common in Group II. Mortality was found to be significant in Group II, especially in DVR (50\%)followed by AVR(23\%). Conclusions:

Approximately 10 percent of the patients had Coronary artery disease. CAG should be rountinely performed in all patients while presenting for valve surgery. Combined valve surgery and CABG carries high mortality (21\%).

\section{MISINTERPRETATION OF MITRAL REGURGITATION IN LEFT ATRIAL MYXOMA COMPLETELY OCCLUDING MITRAL ANNULUS DURING SYSTOLE. Dr Rakesh Kumar, Dr Rani Singh \\ Rajendra Institute of Medical \\ Sciences Ranchi}

\section{Aim:}

Preoperative TTE is not sufficient to look for mitral valve disease in case of left atrial myxoma, TTE is also complimentary to look for it.
Material and methods:

47 yrs old female underwent left atrial myxoma surgery. The mitral valve diseaae was not significant in TTE but it was moderate to severe in TEE. Result:

In case of left atrial myxoma surgery TEE is of utmost importance to verify the mitral valve disease. So it should always be supplemented.

\section{CERVICAL RIB WITH SUBCLAVIAN ANEURYSM \\ Vipin B. Nair, Aravind P. Raman \\ Government medical college trivandrum}

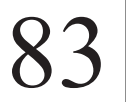

A middle aged gentleman presented with claudication of right upper limb of 6 months duration. He was evaluated with CT/ MRI NECK in view of cervical rib. He was diagnised with vascular type cervical rib- subvlavian artery aneurysm. He underwent cervical rib excision with subclavian artery aneurysm repair using dacron graft. Post operative recovery was excellent. He was discharged on pod 6.

\section{MANAGEMENT OF VARIOUS TYPES OF VENTRICULAR PERFORATION}

Dr Suresh Babu Kale,

Dr Punithakumar Ramasamy,

Dr Chennakesavulu

Meenakshi Hospital, Thanjavur,

Tamil Nadu State, India.

Ventricular perforation, either spontaneous or traumatic, is a serious condition with high morbidity and mortality. Ischemic left ventricular perforations are seen in nearly $4 \%$ of infarcts and present at times with a contained rupture. Tear of the right ventricule due to trauma / stab injury leads to cardiac tamponade and possible death if not identified and repaired in time. Similarly, tear of the right ventricle whilst exposing an intramyocardial left anterior descending artery results in bleeding and if not repaired properly, leads to occlusion of the left anterior descending artery (LAD) and massive anterior wall myocardial infarction. This paper discusses the successful management of these three clinical conditions. Prompt establishment of cardiopulmonary bypass and cardioplegic arrest will assist in achieving good repair and good outcome. A novel sandwich technique is described using the native left anterior descending artery and the bypass graft to repair right ventricular perforation whilst exposing the intramyocardial LAD, avoiding closure of this main artery and preserving graft patency.

\section{TEVAR IN THE TREATMENT OF MULTIPLE PENETRATING AORTIC ULCERS AND RIGHT HEMOTHORAX IN YOUNG MALE OF UNKNOWN ETIOLOGY \\ Dr. Nikhil Dixit, Dr. Amaresh Rao, \\ Dr Shreekant, Dr K Madhusudhan, \\ Dr B Kaladhar, Dr PSS Gopal \\ Nizam's Institute of Medical Sciences, Hyderabad}

After a first description by Shennan in 1934, penetrating aortic ulcer (PAU) is integrated into the acute aortic syndrome. The mechanism of PAU is disruption of the internal elastic lamina that can spread to the media. Arterial hypertension, hyperlipoproteinemia, and aortic sclerosis have shown to be predisposing features of PAU. Because all of these factors are often more present in older patients, PAU is more frequently seen in the elderly. Complicated PAU is defined by the development of aneurysms, pseudoaneurysms, dissections, or aortic ruptures. The first description of TEVAR in the management of PAU was in 1998. We present to you a case of a 32 year old male who presented with massive 
right hemothorax and was evaluated and found to have multiple penetrating aortic ulcers. The etiology was unknown. TEVAR with stenting was carried out and the patient made an excellent recovery

\section{TO STUDY THE AVERAGE DURATION BETWEEN THE ONSET OF SYMPTOMS AND REFERRAL TO A THORACIC} SURGERY UNIT IN NORTH INDIA.

Saraansh Bansal, Ravindra K. Dewan,

Sanam Jindal, Pallavi Purwar,

Ramaswamy Rajendran

National institute of Tuberculosis and Respiratory Diseases

\begin{abstract}
Aim:
Our aim was to evaluate the average duration between the onset of symptoms and referral to a specialised thoracic surgical unit in North India. Methodology:

A retrospective analysis of our database was done of a period ranging from March 2018 to October 2019. All the patients referred to our institute for major thoracic surgical interventions were included in the study. From the records, the presenting complaints with duration and clinical history and examination were noted. A database was compiled and results computed.

Results:

We analysed the records of 320 patients who were referred from various parts of India and subsequently underwent major thoracic surgical intervention. The common presenting complaints were chest pain (48.7\%) and hemoptysis $(45.3 \%)$. Recurrent cough and non-specific fever comprised $18.7 \%$ and $12.8 \%$ of all the referrals respectively. 5 patients $(1.5 \%)$ were referred for chest trauma related complications. The average duration of symptoms was computed. The patients with chest pain had average symptom duration of 11.2 months before visiting our department. Patients with hemoptysis had average symptom duration of 36.26 months while patients with recurrent cough and fever had average symptom duration of 17.5 months and 6.38 months respectively.

\section{Conclusion:}

The major referral to our thoracic surgery unit is of patients with chest pain and hemoptysis. Other reasons are recurrent cough and non-specific fever. Most of these patients are referred months or years after the onset of these symptoms, after receiving multiple courses of anti-tubercular treatment and antibiotics for their symptoms. It is a need of the hour to build a robust thoracic surgery public referral system wherein patients can be referred and treated at the earliest.
\end{abstract}

\section{CABG IN OCTOGENARIANS}

Giridhara Goli, David Varghese

St Bartholomew's hospital

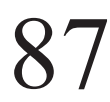

\section{Objectives:}

$\mathrm{CABG}$ is the treatment of choice for patients with triple vessel disease, left main disease and impaired LV function. However, there is a tendency to avoid operating on those over 80 years due to increased mortality and morbidity. We analysed our results of CABG in octogenarians in the last two years.

\section{Methods:}

We did a retrospective review of all patients over 80 who had isolated CABG between August 2017 and October 2019.

\section{Results:}

There were 127 patients. The mean age was 82.3 years (range 80 - 89). $101(80 \%)$ were male. $94(75 \%)$ had triple vessel disease and/or left main stem (LMS) disease. There were 80 (63\%) with good LVEF, 37 (29\%) with moderate LVEF and $10(8 \%)$ with poor LVEF. 55 (43\%) were elective, $69(54 \%)$ were urgent and $3(3 \%)$ had emergency CABG. 93 $(73.2 \%)$ had a preoperative creatinine of \&lt; $80 \mathrm{mmol} / \mathrm{L}, 31(24.4 \%)$ had 80 to $160 \mathrm{mmol} / \mathrm{L}$, and $3(2.4 \%)$ had $\& g t ; 160 \mathrm{mmol} / \mathrm{L} .39$ patients $(31 \%)$ were diabetic. The median body mass index (BMI) was $27.3+/-$ 0.3 . The mean number of distal anastomoses was $2.8+/-0.7$. The mean CPB time was $86+/-24$ minutes with clamp time of $58+/-20$ minutes. 14 $(11 \%)$ had off pump CABG. There were 3 deaths $(2.4 \%)$ postoperatively. 2 patients $(1.6 \%)$ had stroke postoperatively. The median length of postoperative stay was $9+/-0.8$ days.

Conclusions:

$\mathrm{CABG}$ can be performed in octogenarians with excellent results. Age perse should not be a contraindication for CABG.

\section{NON-RESOLVING HYDROPNEUMOTHORAX- AN INTERESTING PRESENTATION}

Dr. Sreekar Balasundaram, Dr. Satish Kumar,

Dr. Nisha Johny

St. John's Medical College Hospital, Bangalore.

\section{Aim/objective:}

To present a very rare case of pleural synovial sarcoma, referred as a non resolving hydropneumothorax.

\section{Material/method:}

A 22 year old lady presented with cough and mucoid expectoration since 2 months. She was subjected to multiple thoracocentesis elsewhere and referred to us for non resolving hydropneumothorax. Further diagnostic evaluation revealed it to be a large cystic lesion arising in the pleura adherent to the lung. Surgical management of this rare case will be presented along with histopathology findings .Data was collected from the patients history, medical records, radiological findings and histopathology with immunochemistry.

\section{Result:}

Patient underwent a successful excision of the tumor and is well on 6 months follow up.

Conclusion:

These rare tumors need a high index of suspicion, and may be inappropriately managed as a non resolving empyema. A good radiological diagnosis, and pathological identification of these rare tumors is warranted.

\section{A CLINICOPATHOLOGICAL}

\section{STUDY OF MEDIASTINAL MASSES} AT OUR INSTITUTE OVER THE

\section{LAST 5 YEARS}

Dr. Sreekar Balasundaram, Dr. Pradeep H P,

Dr. Suresh Kumar, Dr. Nisha Johny, Sinchu Jacob

St. John's Medical College Hospital, Bangalore

\section{Aim/objective:}

To review patients withMediastinal tumors that presented or were referred to our Department over the last 5 years.

\section{Materials/methods:}

Record review of all patients( N-35) admitted with Mediastinal masses to our Institute over the last 5 years were reviewed. Diagnostic methods, OR Records and Histopathology reviews were included.40\% of the Mediastinal masses were discovered incidentally on admission. $60 \%$ of cases were located in the Anterior Mediastinum. Only one case was inoperable on admission. Surgical approaches ranged from mini thoracotomy to sternotomy or a combination of incisions based on pathology and tumour size.

\section{Results:}

Overall postoperative outcomes of these patients were favourable. There was no intraoperative mortality and follow up ranging from 3 months to 1 year were good in terms of freedom from symptoms and negative resection margins. One inoperable case of pericardial mesothelioma and one case of metastatic SCC Cervix (operated) were lost to follow up 


\section{Conclusions:}

Mediastinal masses are rare tumours discovered incidentally most of the times. A high index of suspicion for detection and early referral and interventions will most probably result in a favourable outcome

\section{OUTCOME OF PRE-OPERATIVE LOW DOSE ASPIRIN IN PATIENTS UNDERGOING CABG AN INSTITUTIONAL EXPERIENCE.}

Dr Jaswinder Singh, Dr Ravdeep Singh Sohal

Military Hospital Cardio-thoracic Centre, Pune

\section{Aim:}

To determine the safety and efficacy of Pre-operative Low dose Aspirin administration in Triple Vessel Disease patients undergoing CABG.

Materials and methods:

We conducted prospective case-control study from 01Aug 2015 to 31 Jul 2018, randomly in 200 stable Coronary Artery Disease; Triple Vessel Disease, patients undergoing elective CABG. In Gp I - 100 patients were administered Low dose Aspirin (75mg) and in Gp II 100 patients were studied in which Aspirin was not administered. The safety end point was : more than $200 \mathrm{~mL} / \mathrm{m} 2$ of BSA for $1 \mathrm{st} \mathrm{hr}, 150 \mathrm{ml} / \mathrm{m} 2$ for 2 hrs, $100 \mathrm{ml} / \mathrm{m} 2$ BSA for $4 \mathrm{hrs}$ or $100 \mathrm{ml} / \mathrm{hr}$ for $12 \mathrm{hrs}$, or more than $1200 \mathrm{ml}$ of total chest drain. The patients were followed up for MACE within one month ie; cardiovascular death, recurrent myocardial infarction, repeat revascularization.

Results:

The patients were followed up for 12 months. In Gp I - 19 patients had chest drainage more than $100 \mathrm{ml} / \mathrm{BSA}$ for four hours and 27 patients had drainage more than $1200 \mathrm{ml}$ of total chest drain. In Gp II 13 patients had chest drainage more than $100 \mathrm{ml} / \mathrm{BSA}$ for four hours and 19 patients had drainage more than $1200 \mathrm{ml}$ of total chest drain. In Gp I- nonfatal coronary event (infarction or repeat revascularization, IABP) and major cardiac event (cardiovascular death, infarction, or repeat revascularization, stroke) were $3 \%$ and $5 \%$ in Gp II.

\section{Conclusions:}

Pre-operative Low dose aspirin continuation increased the risk for postoperative bleeding. However, the need for chest re-exploration and the rates of PRBC transfusion and did not increase. On the contrary the rate of MACE was seen less in Gp I.

\section{A RETROSPECTIVE STUDY OF PERICARDIECTOMY IN PATIENTS WITH CONSTRICTIVE PERICARDITIS \\ Prem Prasath Kesavan \\ Christian medical college and hospital, Vellore}

\section{Objectives:}

The aim of this retrospective study was to study the general outcome of the patients undergoing pericardiectomy and associated morbidity and the duration of hospital stay.

Methods:

52 cases of Constrictive pericarditis treated at Christian Medical College and Hospital, Vellore in the last 5 years from October 2014 to October 2019 were analysed retrospectively.

\section{Results:}

Median age of patients was 25 years. Among the 52 patients, out of which there were 38 males $(73 \%)$ and 14(26\%) females. All patients underwent pericardiectomy through a midline sternotomy approach. Tuberculosis remained to be the identifiable cause in most patients [61\%]. The operative mortality was $3.8 \%$ [2 patients], in one patient, he succumbed to low cardiac output due to biventricular failure and in the second patient due to postoperative ventricular fibrillation. Higher preoperative NYHA class symptoms is associated with prolonged hospital stay postoperatively.

\section{Conclusion:}

Pericardiectomy can be carried out as a curative tool in patients with constrictive pericarditis with very good clinical outcome. A higher NYHA class at presentation and presence of ascitis were found to be associated with poor outcome after the surgery. Low mortality is seen in our series due to early intervention in the course of the disease and the use of inotropes in the postoperative period.

\section{D PRINTING \\ (ADDITIVE MANUFACTURING) \\ GUIDED CLOSURE OF A SUBMITRAL ANEURYSM}

Iresh Shetty, prasanna Simha Mohan Rao,

Rakesh Naik Lachma, Prabhu Manohar.

Sri Jayadeva Institute of Cardiovascular Sciences and Research

Submitral aneurysm is a congenital out pouching of left ventricular wall occurring adjacent to the posterior leaflet of the mitral valve. We are reporting a case of a 59 year old gentleman with posterolateral submitral aneurysm who underwent aneurysm patch repair and mitral valve repair. A good knowledge about the interrelationship between aneurysmal sac and mitral valve was obtained enabling proper surgical repair. A careful and well organized pre-operative planning, cardiac MRI along with a 3D printed model gave a good understanding of orientation and hence avoided any surprises intraoperatively. The importance of 3D printing in determining the exact location and dimensions of the aneurysm and patch size is highlighted. Successful surgical repair depends on accurate understanding of relationship between mitral annulus, valve, and aneurysmal sac.3D printing provided that information and helped plan repair

\section{AORTIC CUSPS RE-IMPLANTATION WITH GLUTERALDEHYDE TREATED PERICARDIUM IN YOUNG FEMALES} Sujoy Chatterjee, Srirup Chatterjee,

Rajarshi Ghosh, Santosh Kumar Pandey, Lakshmi Sankhayan, Nikhil Bansal, Satyajit Bose

The Mission Hospital, Durgapur

\section{Introduction}

Rheumatic Aortic Valve disease is prevalent in rural and semi-urban Indian population. The harmful effects of oral anticoagulants on pregnancy and child birth especially in this subset of patients is very common. This study aims to determine the feasibility of aortic cusps reconstruction by Modified Ozaki's technique in these patients.

\section{Materials and Methods}

Aortic cusps reconstruction was performed in 43 female patients between April 2012 to March 2019. 25 underwent repair of isolated Aortic Valve and remaining both mitral and aortic valves. 30 out of 43 had mixed leisions AS \&amp;AR typical of RHD, 6 had isolated AR and 7 pure AS. 5 of these AS patients had congential aortic stenosis, 4 of which had bicuspid aortic valve. The results were reviewed retrospectively. Mean age of these patients was 12.2 years ( range 7.8 years to 17 years). Preoperative echo revealed a mean peak pressure gradient of $60.3 \mathrm{~mm}$ of hg ( range 40 to $75 \mathrm{~mm}$ of hg). Surgical annulus diameter was $19.4 \mathrm{~mm}$ ( range 16 to $24 \mathrm{~mm}$ ). The surgical procedure included excision of native leaflets and clearing the annulis of calcium if any. Measuring the annulus with sizer. Sizing the fixed pericardium such that 3 semicircular neocusps are made each having diameter of the annulus. Suturing the circular margin of the cusps along the annulus while the straight side acts as coapting surface. All patients were kept on aspirin and oral rheumatic prophylaxis.

\section{Results}

Mean follow- up was 46 months with maximum of 6.5 years. There was no in hospital mortality. Post op echo revealed an average peak gradient of $15.1 \mathrm{~mm}$ of hg ( range 12 to $20 \mathrm{~mm}$ of hg) with nil to mild AR at 
discharge. 7 patients required AVR in this follow-up period, earliest at 6 months of surgery due to infective endocarditis and others had recurrence of rheumatic activity and developed severe AR. There were no thromboembolic events. 10 out of these patients had uneventful child birth in the follow up period.

\section{Conclusions}

Treating aortic valve leisions by aortic cusps re-implantation is feasible for select sub set of patients who have contraindication for anticoagulation or are aspiring mothers.

\section{INTERIM ANALYSIS OF RANDOMIZED CONTROLLED STUDY OF SHORT-TERM OUTCOME OF USAGE OF DEL NIDO VS BLOOD CARDIOPLEGIA IN ADULTS UNDERGOING HEART VALVE REPLACEMENT \\ Dr Aravind Kalyanasundaram, \\ Sreevathsa K S Prasad, \\ Ramsankar Padmanaban, \\ Hemachandren Munuswamy, \\ Durga Prasad Rath, Saichandran BV \\ JIPMER}

\begin{abstract}
Aim/Objective:
To assess short term clinical outcomes based on perioperative troponin $\mathrm{T}$ levels (before start of surgery, 2 hours and 12 hours after coming of $\mathrm{CPB}$ ) of adult patients undergoing cardiac surgery using the del Nido cardioplegia technique compared with stthomasconventional blood cardioplegia

Material and method:

This was a prospective randomized study with a sample size of 50, which included patients with valvular heart disease requiring single valve replacement in our center from February 2019 to November 2019.Simple randomization technique was used and patients were divided into two groups. perioperative TROPONIN levels, TEE ejection fraction, postoperative inotrope requirements were analyzed.

\section{Results:}

Total of 50 Patients were studied ofwhich31 were female and 19 male. Mean age was 37 (18-67). These patients were divided into two groups 27 cases received blood cardioplegia and 23 delnidoplegia. Meantroponin T immediate post CPB was 487 in delnido and 514 in blood showing no significance (P 0.354$)$ and $24 \mathrm{hrspost}$ surgery where 267 and 210 respectively with no significant $(0.093)$ difference. There was a significant $(\mathrm{p}-0.002)$ difference in the number days patient required inotrope post-operatively. Other parameters like coming of pump need for defibrillation(p-0.78), change in $\mathrm{EF}$ measured pre and post $\mathrm{CPB}(\mathrm{p}-0.437)$ did not show any significance.

\section{Conclusion:}

Myocardial protection in open cardiac surgery is still evolving. Delnidocardioplegia is a viable alternative tostthomascardioplegia which has proven to be statically equivalent in myocardial protection in adult population also. Further studies are required to look into the longtermoutcome of use of delnidoand to expand the use of delnidocardioplegia in other adult open cardiac surgeries.
\end{abstract}

\section{MINIMAL INVASIVE CARDIAC SURGERY (MICS) WITH ROUTINE INSTRUMENTS AND INDIGENOUS TECHNIQUES.}

Dr. Frankleena Parage,

Dr. S S Lohchab

Postgraduate Institute of medical sciences, Rohtak

\section{Introduction:}

Minimal invasive cardiac surgery is defined when it is sternal sparing procedures or with minimally invasive CPB sparing procedure.
Minimal invasive cardiac surgery is popular due to its various advantages like minimal blood loss, reduced trauma, less postoperative pain, decreased duration of mechanical ventilation, cosmetically better scar, short hospital stay, quicker return to normal activities etc. Both off pump and on pump surgeries can be performed via minimal invasive cardiac surgery. Methods:

MICS can be performed via mini thoracotomy, upper/lower sternotomy, video assisted and total endoscopic or robotic surgery. However MICS can be performed in selected patients only and it may be difficult for the surgeon to perform it. From year 2010 to 2019, MICS was being performed in our institution without any specialised instruments and with modification of techniques.

Results:

At our institute we have been performing various procedures through minimal invasive approach, which includes mitral valve repair/ replacement (42 patients), tricuspid valve repair (34 patients), aortic valve replacement (6 patients), ASD closure (54 patients), VSD closure (6 patients), coronary artery bypass grafting ( 7 patients) and cryomaze ablation for atrial fibrillation (4 patients). TV repair and cryomaze procedures were performed concomitant to mitral valve surgery 2 patients underwent Aortic valve replacement through upper hemi sternotomy and 4 through right mini thoracotomy through 2 nd intercostal space. These following procedures were performed in 115 patients at our institute. There was no post operative mortality noted in these patients. One patient with AVR was explored post operatively view of excessive bleeding. The average blood loss was approximately $200 \mathrm{ml}$ in the patients undergoing MICS. On table extubation was performed in 10 patients. The duration of mechanical ventilation was minimised on an average to approximately 3 hrs. The patients experienced less pain as compared to the conventional full sternotomy approach, The average length of the post operative hospital stay was 3 - 4days. The conversion of minimal invasive cardiac surgery to conventional midline full sternotomy approach was done in one patient.

\section{Conclusion:}

The minimal invasive cardiac surgery can be performed safely via mini thoracotomy or upper mid sternotomy approach with the routine instruments and indigenous techniques.

\section{SINGLE VS DOUBLE INTERNAL} MAMMARY ARTERY IN CABG

Prof. Dr. James Thomas,

Dr. Abhay Jain, MD, Dr. Mahesh Singh,

Dr. Umbarkar S

D.Y. Patil University and Hospital,

3rd Floor Administration, Sector 5, Nerul,

Navi Mumbai 400706

\section{Objective :}

Coronary Bypass grafting is highly effective for relief of symptoms in multi vessel and left main coronary artery disease. Superior patency of Internal mammory artery grafting compare to saphenous vein in conclusive. Left IMA is established as standard care for grafting the LAD. The aim of presentation is to compare early outcomes of patients receiving single IMA vs Bilateral IMA.

\section{Method :}

Between 2008 and 20191422 patients underwent CABG using single IMA in addition to vein grafts and radial artery grafts in few cases. Bilateral IMA was performed in 840 patients (Drs. MS and RU) during the same period. Youngest patient to receive single IMA was 34 years female and 35 years male and the oldest was 77 and 88. Male female ratio 60:40 and $45 \%$ of patients were below 60 years. Incidence of diabetes in both group were $70 \%$. One of the CABG in single IMA group had a mobile thrombus near LV apex and was removed. And 4 patients had post infarction VSD repaired in addition to CABG. Preoperative use of IABP in low ejection fraction patients were done in 20 patients. $80 \%$ of all anastomosis in the SIMA group was done on cardio pulmonary bypass. The cardioplegic arrest both anti grade and retro grade group. Skeletonization of RMA was done in the BIMA group mostly Y 
anastomosis with RIMA to LIMA. Vein grafts were mostly single anastomosis. Mortality in single vs double mammory was $7 \%$ and $8 \%$. There was no significant difference in perioperative MI, Renal failure and pre operation for bleeding. Slight increase in mediastinitis $3.2 \%$ in BIMA group. Coronary Angiography and CT Angiography was done post operatively. 2 patients had single IMA and 2 Vein grafts patent at 10 years post operative.

Conclusion : Low use of Bilateral IMA is still prevalent in many countries. Increased technical complexity, incidence of diabetes mellitus, diffuse diseases, potential increase in mortality and morbility. Single IMA with vein graft and additional radial grafts is still the preference with good results for many surgeons. Retro grade cardioplegia in addition to anti grade cardioplegia in On Pump patients gives good results. With experience $\mathrm{CABG}$ using Bilateral IMA can still be performed with good clinical results and low mobility.

\section{APPLICATION OF CARDIOPULMONARY BYPASS IN NON CARDIAC SURGERY-A 10 YEAR RETROSPECTIVE STUDY AT NIMS HYDERABAD \\ Sohail Tarekh Sayyad, R.V Kumar, \\ T. Ramakrishna, Sai Surabhi \\ Dept of Cardio Thoracic Surgery, NIMS Hospital, Hyderabad}

\begin{abstract}
Aims and objectives:
Cardiopulmonary Bypass (CPB) are used to facilitate circulatory support in standard cardiac surgery and emergency intervention, but CPB are not used routinely in non-cardiac surgery include isolated limb perfusions in cases of malignant melanoma, lower tracheal surgeries, thoracic tumour resection, aortic pathology and lesions involving the great vessels, neurosurgery for aneurysms and tumours, urological surgery for renal cell carcinomas. The primary aim of this study was to identify the type of non-cardiac procedures and bypass used in our institution and review the patient outcomes including perioperative and bypass complications.

Methods:

A retrospective study was performed within the Cardiothoracic Surgery Unit (CTSU) of NIMS Hospital, Hyderabad that examined 20 non cardiac cases between 2009 and 2019 in which CPB was used. Cases excluded from the study were thoracic aneurysm and pulmonary embolectomy with the use of CPB.

Results:

Twenty non-cardiac surgery cases were reviewed using CPB support. The non-cardiac surgical procedures included eight isolated limb perfusion cases for malignant melanoma ,four tracheal cases, three renal cases ,four mediastinal cases and one neurosurgical case. No direct complications of CPB were noted.

Conclusions:

Our review of 20 cases managed under the CTSU has shown that CPB can be used in a range of oncological, thoracic, neurosurgical, renal and tracheal surgery. These surgical procedures have involved the management of haemodynamically unstable patients. Patient outcomes have been encouraging with few complications. With further research including the use of a larger sample size and control groups, more definitive conclusions could be made on the benefit of $\mathrm{CPB}$ to patients in non-cardiac surgery
\end{abstract}

\section{RT. AORTIC ARCH WITH MIRROR IMAGE BRANCHING WITH DOUBLE DUCTUS ARTERIOSUS AND LPDA CONTINUOUS AS LPA AND RPA AS A CONTINUATION OF MPA a rare variety \\ Dr. Divyesh Rathod, DR Amit Mishra, \\ Dr Jigar Shah \\ U N Mehta Institute of Cardiology \\ and Research Center}

Rt. Aortic arch with Double ductus arteriosus with anomalous origin of the left pulmonary artery from left pda is a rare congenital heart disease. Echocardiography is the first-choice method to diagnose pulmonary arteries and presence of the arterial ductus. Computed tomography (CT) angiography confirms the final diagnosis of the anomalous origin of the pulmonary artery. We report a case diagnosed using 128-slice CT angiography where this case demonstrated presence of both ducts and an anomalous origin of left pulmonary artery from the left pda .Learning objective: Anomalous origin of the left pulmonary artery usually presents in early infancy with heart failure and rapid development of pulmonary hypertension. An association of two patent arterial ducts and an anomalous origin of the left pulmonary artery from the left -patent arterial duct is a rare finding. Computed tomography angiography is the best diagnostic modality to establish the correct diagnosis, which is crucial for further decision-making and optimal patient management early surgical correction includes ligation of both ducus and lpa reimplantation is definitive management

\section{ANNULAR CONSTRICTIVE PERICARDITIS CAUSING HOUR-GLASS CONTRACTURE OF THE RIGHT VENTRICLE.}

99

Dr. Utkarsh Sanjay Sanghavi, Dr. Anagha Tulsi A;

Dr. Hiremath CS; Dr. Shekhar Rao

Sri Sathya Sai Institute of Higher

Medical Sciences, Bengaluru

\section{Introduction:}

Constrictive pericarditis $(\mathrm{CP})$ is characterized by the encasement of the heart by a rigid non-pliable pericardium due to dense fibrosis and adhesions. Prevalence of $\mathrm{CP}$ is reported in $0.2-0.4 \%$ of patients following cardiac surgery and it can also occur after pericardial trauma or inflammation due to a variety of etiologies. Case report:

A 13-year old male presented with complaints of shortness of breath restricting his daily activities with a history of recurrent abdominal distension and pedal edema for three years which got relieved with diuretics. TBPCR was negative. On examination, JVP was raised with $15 \mathrm{~cm}$ from sternal angle with prominent y descent and Kussmaul's sign was positive. Trans-thoracic echocardiography showed thickened pericardium, septal bounce and normal biventricular function. CT chest with contrast showed loculated peripherally calcified collection measuring $23 \times 36 \times 58 \mathrm{~mm}$ over the right ventricle free wall with tubular configuration of the right ventricle and dilated IVC and hepatic veins. On median sternotomy, the pericardium was heavily calcified with calcium speckles invading the myocardium. It was densely adhered to the RV causing hour-glass constriction of the RV. Also, there was small cavity of localized caseous material over the anterior aspect of RV. Off pump Total pericardiectomy (phrenic to phrenic) was performed. During the procedure LAD was injured which was repaired on beating heart using octopus stabliser. The histopathology report revealed necrotizing granulomas compatible with tuberculous pericarditis.

Conclusions:

Annular CP though a rare form of CP should not be overlooked and can be successfully managed with off pump total pericardiectomy.

\section{CFD ANALYSIS OF DESIGN, FLOW OF ENCLOSED IMPELLER OF SAISPANDAN-THE INDIAN MAGNETIC BEARING-LESS ARTIFICIAL HEART}

Pradeep Kumar Radhakrishnan, Sujatha

Mohanty, Pulivarthi Nageshwar Rao,

Sivakrishna Rao G V, Nagesh Kumar, SivaPrasad,

Satyanarayana Murthy, Bisoi A K,

Satyanarayana M R S, Ravishankar, Srinivas,

Das P K, Valluvan Jeevanandam, Venugopal P

GIMSR, Gitam University, AP 


\section{Aim/Objectives}

Saispandan is an indigenous Indian total artificial heart being designed in Gitam University, India. It works on the principle of miniaturized hybrid 12/14 bearing less switched reluctance motor. Flow simulation and bio compatible impeller designing of the magnetically levitated bearing less pump is being done using computational fluid dynamics (CFD) software to obtain optimal design for the desired flow characteristics.

\section{Material and Methods}

Three dimensional computational domain consists of impeller with casing will be created and meshed using unstructured mesh with the prism layer to capture flow pattern near the wall in ICEM CFD 15.0 software. The boundary condition at the eye inlet of impeller will be supplied with 5 $1 /$ min mass flow rate .Governing equations continuity, momentum, energy equations and laminar flow will be solved under steady conditions to analyze the blood flow and temperature distribution in the impeller using FLUENT 15.0 software. Finite volume method will be used for the discretization of governing equations and pressure velocity coupling will be performed by using the semi-implicit method for pressure linked equation (SIMPLE) algorithm. The effect of bio compatible material on blood flow pattern and temperature distribution within the impeller will be analyzed. Results will be presented in terms of uniquely colored velocity path lines, isotherms and the influence of pertinent parameters (like impeller geometry parameters) on abnormalities will be evaluated.

\section{Results}

Parametric evaluation of flow separation, back pressure, temperature distribution would identify most suitable bio compatible material for impeller designing and incorporation into BSRM circuit. Any difference in obtained and predicted head at desired flow rates would be noted. At present modeling and simulation work is in progress in central research lab of GITAM.

\section{Conclusion}

Inlet and outlet angle and number of blades, for maximal pump efficiency would be noted. Simulation will be completed in a weeks time as per phase II of the Saispandan : The Gitam Heart TAH roject. Results of impeller CFD analysis with optimal design will be presented.

\section{EXPERIENCE WITH CARDIAC MYXOMAS (RARE BENIGN TUMOR) AT OUR CENTER: A PROSPECTIVE STUDY AND ANALYSIS}

Dr. Vaibhav Chugh, Dr Dhananjay Bansal (Assistant Prof), Dr Narender Singh Jhajhria (Prof),

Dr Vijay Grover (Prof \& HOD),

Dr Vijay Kr Gupta (Prof)

ABVIMS and Dr. RML Hospital, New Delhi

Experience with

\section{Objectives:}

Cardiac myxomas are the most frequent cardiac tumors. The aim of study is to describe the incidence, clinical symptoms, investigation findings and surgical management of cardiac myxomas.

Methods:

From July 2015 to July 2017, 15 patients of primary intracardiac myxoma underwent surgical excision at our institute. Their age ranged from 40 years to 65 years with predominance of female patients (73.3\%). Surgical resection was done in all the cases via biatrial approach or right atriotomy. Postoperative echocardiogram was done in all patients before discharge. Maximal follow-up of two years and minimum follow-up of 6 months was done after surgery.

Results:

The tumors presented in a variety of ways, namely, dyspnea $(66.7 \%)$, chest pain $(26.27 \%)$ and transient ischemic attack $(6.6 \%)$, being the common modes of presentation. The most common location for the tumor was the left atrium (80\%) with the fossa ovalis being the most common
$(66.7 \%)$ site of attachment. No mortality or any significant morbidity was found. On follow-up all patients were in NYHA class 1 and their echocardiography showed good ventricular function with normal pulmonary artery pressure with patch in situ. No recurrence was reported till date.

Conclusion: Early diagnosis and surgical resection of cardiac myxomas contributes to excellent prognosis and associated with low complications and recurrences rate. Regular long-term follow-up should be done in all patients with cardiac myxomas.

\section{AN INNOVATIVE INDIGENOUS PORTABLE-WEARABLE \\ DEVICE TO MEASURE \\ ELECTRO-SEISMOCARDIOGRAM} AND CARDIAC STRAIN PARAMETERS.

Manav Bhushan, Ph.D,Kirthi Vidyasagar, MD;

Peter Joseph, MBBS; Ravali Amba, M.Tech;

Sandeep Sibal, Ph.D; Aditya N Doddamane, B.Tech;

Prayaag Kini, DNB; Reeta Viryani, DNB;

CS Hiremath, MCh; Shekhar Rao, MCh

Sri Sathya Sai Institute of Higher Medical Sciences, Whitefield, Bengaluru, India. 560066

\section{Background:}

Mobile monitoring of patients both prior to and after surgical intervention for rhythm disturbance(s) and structural cardiac defects across the congenital and adult age groups have been a step forward in remote monitoring, tele-health medicine and a disruptive change in communication technology. We present a novel interface that is built on the premise of bringing diagnostic tools at the fingertips of healthcare professionals and miniaturizing interfaces that are both patient-affable and safe.

Material and methods:

HeartOs is a novel indigenous non-invasive cardiac health monitor (developed by Fourth Frontier Technologies) weighing $\sim 20$ grams, and worn on an elastic chest strap, meant for accurate ambulatory measurements of Systolic Time Intervals (STIs) using the Seismo cardiogram (SCG) signal, and single-lead Electrocardiogram (ECG) in subjects with cardiac health problems as well as sports enthusiasts and health-conscious individuals. The device provides the user and physician easy access to valuable health data (Heart Rate, Breathing Rate, STIs- PEP/LVET and ECG, ST-segment analysis and arrhythmia detection), allowing for reliable recognition of variation in cardiac function thereby facilitating early intervention and timely management. This data can be easily uploaded to a secure server by the subject themselves, using a mobile application, the wireless data transfer eliminates the need for the patient to come back to the Hospital/clinic for frequent follow-ups. A unique aspect of HeartOs is that it has an in-built processor, which is capable of detecting abnormalities in the ECG and the STIs calculated from a combination of the ECG and the SCG in real-time, on the device itself, and subsequently alerting the User with a discrete vibration alert. The device can also live-stream ECG and SCG signals to a mobile phone.

\section{Conclusion:}

Valuable data on the Systolic time intervals - Pre ejection period (PEP) and the Left Ventricular Ejection Time (LVET) - can be obtained by combining the ECG and SCG analysis, and can be used to assess left ventricular dysfunction. This wearable device can facilitate real-time monitoring of cardiac contractility (using PEP and LVET) in a mobile setting, and this can be used to track the cardiac status of patients with heart disease at home. The SCG tracings evaluated by an automated proprietary algorithm in this wearable have been shown to correlate well with the PEP and LVET values calculated from an Echocardiogram in patients with and without LV dysfunction, opening up new avenues for ambulatory monitoring of these parameters Currently, two studies at SSSIHMS, Bangalore have been approved by the institutional ethics board which will look at validating arrhythmias and STIs obtained in 
subjects with congenital heart defects and in subjects with underlying aortic pathology.

\section{INTRATHORACIC IVC BULLET INJURY - A RARE CASE \\ Dr Poornima Sivakumar \\ Apollo Gleneagkes Hospital, Kolkata}

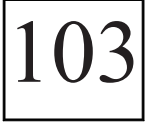

AIM : In cases of emerging chest trauma and major vascular injuries, immediate surgery to prevent patient exsanguination and also as a life saving measure.

MATERIAL AND METHODS: A 40 year old male weighing 110kgs came with alleged H/O Bullet Injury through left chest at 12 noon. Patient presented to our ER at3pm with pain at the injury site and mild breathlessness. On examination patient was conscious, oriented, haemodynamically stable with a BP-130/84 MMHG, PR-78/min and SpO2 - 98\% in room air. Entry wound was seen at the left 4th Intercostal Space with no exit wound. Chest X-Ray PA and Lateral view revealed the presence of the bullet on the left side of the chest above the left hemidiaphragm. Trans thoracic ECHO and CT Thorax showed the bullet close to the Right Ventricle and IVC with minimal pericardial effusion and an EF - 55\%. TEE was done to further reconfirm the position of the bullet which revealed no intrapericardial Haematoma, no significant pericardial effusion and the presence of the bullet close to RV/IVC but couldnâ $€^{\mathrm{TM}_{\mathrm{t}}}$ further localise due to extreme echogenic artefact of the metallic shadow. Patient relatives were counselled for emergency evacuation of the bullet and management of any intrathoracic injury. OT started at $7.30 \mathrm{pm}$ with 8 staffs including the perfusionist. We had kept the cell saver ready in anticipation of cardiac chambers injury and subsequent significant bleeding. Post sternotomy there was frank blood in the pericardial cavity which was immediately retrieved through the cell saver. In anticipation of haemodynamic instability, preliminary arrangements for establishing CPB was done along with systemic heparinization. As the heart was lifted, bullet was visualised close to IVC and the moment it was retrieved from the surgical field, there was a sudden gush of venous blood and the systemic BP which was initially $130 / 70 \mathrm{mmHG}$ became $10 \mathrm{mmHG}$. Immediately $\mathrm{CPB}$ was instituted and the shed blood was returned to the oxygenator within couple of minutes. We then assessed the nature and extent of the injury. To our surprise we found a $2 \times 2 \mathrm{cms}$ tear was found at the antero-lateral aspect of the wall of IVC extending till the right dome of diaphragm. We then encountered a technical problem of air trapping in venous line due to this large IVC defect which was not allowing the full flow CPB maintenance. This was sorted out by prompt Right Femoral Venous cannulation. Inspire of full $\mathrm{CPB}$ we still couldnâ $\mathfrak{E}^{\mathrm{TM}_{\mathrm{t}}}$ isolate the IVC Injury site due to the continuous leak. We then instituted TCA and patient was called down to 18 degree $\mathrm{C}$ with optimal Neuro protection from the anaesthetist end. A secured repair of IVC tear was done using a pericardial patch within 25 minutes. Patient was then taken out of TCA and circulation was established using CPB. To our surprise, another linear tear $2 \times 2 \mathrm{cms}$ was found near the posters-medial border extending to the diaphragm. As it was risky to go into TCA again in view of the compromised cerebral protection, this repair was carried out with low flows under CPB Bypass. The defect was repaired directly with $4 \hat{a} €^{\mathrm{TM}_{\mathrm{O}}}$ prolene. Patient was gradually weaned off $\mathrm{CPB}$ and the total surgical procedure lasted almost 6 hours. Patient wa shifted in a haemodynamically stable condition to our CTVS ICU with very minimal ionotropes.

RESULTS : 2 hours post surgery patient was wide awake and obeying commands indicating the status of proper Neuro protection . Patient was gradually weaned off the ventilator and ionotroped on the 1st Post op day and he recovered well over the next few days. Patient was discharged on the 5th Postoperative day in a stable condition.

CONCLUSION : IVC injuries are uncommon and the mortality rate is extremely high despite the advancements in technical and organisational skills. The most important prognostic factors are the extent of haemorrhaging shock and site of injury (Supra or Infra Diaphragmatic).
Supra Diaphragmatic and Retro Hepatic IVC injuries have the worst prognosis and a very high mortality rate even before presenting to the ER in view of the inaccessible location of the injury and the complex interventions required. IVC injuries have a mortality rate of almost 70-90\%. Death is mainly due to exsanguination or cardiac tamponade. There are very few cases reported so far in literature due to the increased mortality of such patients even before they present to the ER. Supra hepatic interventions are employed for unstable patients and also for ones with a large IVC defect. It is a highly challenging surgical intervention in view of the location and the complexity of the repair involved. There is only one such reported case of successful supra diaphragmatic IVC injury repair in literature so far. We herein present the second successful case of IVC injury repair.

\section{RARE CASE OF RECURRENT GIANT CELL TUMOR OF LUNG \\ DR. PHANI KRISHNA M K,}

Dr. Saravana Krushna Raja, M.Ch,

Dr. Joseph Raj, M.Ch

Madras Medical College

Giant cell tumors of soft tissue are rare tumors that demonstrate a spectrum of benign to malignant potential. Although the majority of these tumors are located in the lower extremity, they may occur rarely in the posterior mediastinum and present as a posterior mediastinal mass with good prognosis after surgery. Giant cell tumours are commonly seen in bone and soft tissues. Primary mediastinal giant cell tumor of soft tissue in the English literature was published by Fu et al. in 2002, in which they described two patients with posterior mediastinal masses. Benign cystic tumor of lung is rare and has not been reported in literature earlier. Giant cell tumors are normally benign with unpredictable behavior. It is characterized by the presence of multinucleated giant cells( osteoclast like cells). Malignancy in giant cell tumor is uncommon and occurs in approximately $2 \%$ of all cases. We are now reporting this case as first case in our country. Surgical treatment is curative. We noticed recurrence in one year's time and the patient had to undergo Pneumonectomy after the initial lobectomy.

\section{A NOVEL EXTRACORPOREAL KNOT-TYING TECHNIQUE IN MINIMAL ACCESS SURGERY}

Dr Gaurav Patni, Dr Lalit Kapoor,

Dr Pradeep Narayan, Dr Devraj Kumar

NHRabindranath Tagore International

Institute Of Cardiac Sciences, Kolkata

Aim: A wide variety of techniques has been described for extracorporeal knot tying. However there is no universal acceptance of any particular technique. We aimed to describe an extracorporeal knot-tying technique that does not require any special instruments, has an extremely short learning curve, does not cause fraying of sutures, and because of its simplicity has the potential for universal acceptance.

Material and Methods: The knot is tied extracorporeally in a conventional way. While one limb of the suture is held as in the conventional knot-tying technique, the other limb above the knot is passed through laser-drilled holes in prefabricated polytetrafluoroethylene (PTFE) pledgets which are used in valvular surgery very commonly. Holding the PTFE pledget by any long instrument like a hemostatic forceps the knot is pushed down. The process is repeated until the desired numbers of knots are achieved.

Results: In this technique there is no contact between the suture and the instrument at any point and the extracorporeally tied knots can be easily levered down. . The time taken is an extra 10 seconds compared with conventional knot tying. The learning curve is limited to a few knots and does not require any special equipment. We have used this technique in 
coronary surgery, aortic valve replacements, and in mitral valve surgery. After an experience of 10 knot-tying, the incidence of accidental suture breakage is comparable with conventional finger knot tying.

Conclusion: The ease and safety of the technique allows it to be taught to all surgeons irrespective of experience. This technique is simple, safe, and easy to master and has the potential for universal acceptance.

\section{ACQUIRED COR TRIATRIATUM, TO RE-ENDOTHELIALIZE THE UNROOFED CORONARY SINUS OR NOT? \\ Dr Dinesh D, DR B R Jagannath, \\ Dr Kvss Saikiran, \\ DR Gopichand Mannam, DR Sairam, \\ DR Ramkinkar Shastry \\ Star Hospital, Hyderabad}

\section{Introduction}

Repair of cardiac type of TAPVC consists of unroofing the coronary sinus (CS) into the left atrium and creating an unobstructed path from the pulmonary vein orifices to the mitral valve to prevent obstruction. To avoid possibility of going out of the heart, there is need for reendothelialization of the area by continuous/interrupted suture.

Case report: 10-month-old child was operated for cardiac TAPVC where the unroofing of coronary sinus was done along with reendothelization with pericardial patch closure of ASD. One year later he presented with heart failure symptoms. On evaluation, found to have obstruction at anastomotic site of LA with obstruction between the pulmonary vein orifices and the mitral valve as would be seen in a Cor triatriatum. Intraoperatively all pulmonary veins were draining into LA through a small slit with fibrous fusion of unroofed portion of coronary sinus, which was adherent to IAS patch, causing obstruction to PV drainage. The reendothelialized part had constricted, due to purse string effect or fibrosis. The ring of fibrotic tissue was excised and the pulmonary vein orifices were draining normally into the mitral valve. Resulting ASD was closed with another pericardial patch. Intraoperative and postoperative periods were uneventful. Echo showed unobstructed pulmonary venous drainage. Patient discharged on $7^{\text {th }}$ POD.

Conclusion: While reendothelialization using continuous suture make sure that the purse string effect is avoided. The suture should be tied to itself at both ends, and avoid attaching to the patch suture as it tends to purse string when pulled up, which is probably what happened in our case.

\section{TOPICAL VANCOMYCIN IN CONJUNCTION WITH PERIOPERATIVE ANTIBIOTICS AND TIGHT GLYCAEMIC CONTROL HELPS TO ELIMINATE STERNAL WOUND INFECTIONS \\ Dr. Noaman Shaikh, Dr. Arnab De, \\ Dr. Tejas Dandekar, Dr. Vaibhav Shah, \\ Dr. Manish Jadhao, Dr. Chaitanya Raut, \\ Dr. Prashant Mishra, Dr. Jayant V Khandekar. \\ Lokmanya Tilak Municipal Medical College \\ and Hospital, Sion, Mumbai.}

Aim:To study whether topical vancomycin would further reduce the incidence of sternal infections in the presence of perioperative antibiotics and tight glycaemic control in patients undergoing CABG.

Methods:A retrospective analysis of 349 consecutive patients undergoing CABG from August 2017 to November 2019 receiving topical vancomycin ( $2.5 \mathrm{~g}$ in $2 \mathrm{~mL}$ of normal saline) applied as a slurry to the cut edges of the sternum were compared with 356 patients who did not receive topical vancomycin. All patients received perioperative antibiotics on anaesthetic induction which was continued for 48 hours; and intravenous insulin infusions to maintain serum blood glucose level between 120 and $180 \mathrm{mg} / \mathrm{dL}$.

Results:Patients receiving topical vancomycin had less superficial sternal infections (1.14\%vs 7.02\%;P\&lt;.001), deep sternal infections $(0.57 \%$ vs $1.12 \% ; \mathrm{P}=0.001)$. Patients with obesity (BMI\&gt; 30 ) showed a lesser rate of infection in the vancomycin group $(1.92 \%$ vs $6.84 \%)$. The vancomycin group also showed lesser rates of sternal infection in patients with diabetes $(2.02 \%$ vs $7.3 \%)$. When patients with diabetes and obesity were considered, vancomycin group showed a significantly lower rate of sternal infections $(2.11 \%$ vs $6.56 \%)$.

Conclusions:Topical vancomycin applied to the sternal edges, in conjunction with perioperative antibiotics and tight glycaemic control, helps to eliminate wound infections in patients undergoing CABG.

\section{AORTIC INTRAMURAL \\ HEMATOMA-HYBRID \\ APPROACH- A CASE REPORT}

DR Dinesh, DR Gopichand Mannam,

DR D S Bhaskar Raju,

DR Satyendranath, DR Kvss Saikiran

Star Hospital

\section{Introduction}

Aortic intramural hematoma (IMH) accounts for $10-30 \%$ cases of acute aortic syndrome. Although less common, IMH has identical clinical manifestations, risk factors, clinical courses and treatment principles to aortic dissection. Without prompt recognition and proper management IMH can also result in high mortality.

Case report

75 yr old male patient presented with chest pain associated with sweating for 2 days. Had abdominal discomfort associated with vomiting. He went to nearby hospital where patient was evaluated for MI. Trop-T â€"ve. No ST-T wave changes. On suspicion of aortic dissection, CT chest and abdomen showed ?type B aortic dissection with left renal infarct. Referred to our hospital for further management. On admission patient developed AF which was managed with cardarone. Patient was hemodynamically stable. Hence CT aortogram was repeated which showed intramural hematoma involving ascending aorta, arch and extending into descending and abdominal aorta. Aortic root was normal. Echo showed normal aortic valve with normal LV function. Known hypertensive on treatment. Old CVA 5years back. Intraoperatively intramural hematoma involving the ascending aorta, arch extending into descending aorta. No intimal tear/rent noted. Underwent supracoronary ascending aorta replacement wit Dacron tube graft with debranching of arch vessels with cooled to $28 \mathrm{C}$ andusing antegrade cerebral perfusion. On POD-1 thoracic endovascular aortic repair was done using $34 \times 30 \times 200 \mathrm{~mm}$ stent through right femoral approach. Patient extubated on day 2. Post op period uneventful except for 3 episode so AF. Patient discharged on 9th POD.

\section{Conclusion}

Because patients with aortic intramural hematoma can develop, aortic rupture, dissection or aneurysm early intervention is necessary to prevent complication.

\section{HAND-MADE VALVED PTFE RIGHT VENTRICLE TO PULMONARY ARTERY CONDUIT - HOW DO WE DO IT?}

Dr. K. Srikanth, Dr. Sudesh Prabhu

Narayana Hrudayalaya, Bangalore

Introduction Right ventricle to pulmonary artery conduits are necessary in many cases of Tetralogy with pulmonary atresia or with a major coronary crossing RVOT, Truncus repair, Rastelli operation to name a few. Many such patients receive either a homograft conduit which has its inherent problem of speedy degeneration or a synthetic readily available 
commercial conduit which is expensive. In this video, we demonstrate how we fashion our own valved RV to PA conduit using a PTFE tube and PTFE membrane. AimTo share our technique of preparing the valved PTFE RV to PA conduit and to demonstrate how it is incorporated in a case of Tetralogy with pulmonary atresia. Conclusion The handmade valved PTFE RV to PA conduit is a very simple, economical and reproducible technique which will help surgeons overcome the shortcomings of a homograft or a commercial conduit. The mid-term results of the PTFE conduit is very promising in terms of degeneration rate, incidence of infective endocarditis and the overall outcome of patients with this conduit. We hope this video would encourage fellow surgeons to incorporate this valved conduit into their daily practice when faced with such cases requiring a RV to PA conduit.

\section{ASD OCCLUSION BY A STERNUM SPARING TRANSTHORACIC} MINIMALLY INVASIVE APPROACH

Dr Sanjay Kumar, Dr Vladamir Shumaster 1 Department of Cardiothoracic Surgery, Institute of Medical Sciences, Banaras Hindu University, Varanasi, India; 2 Division of Cardiac Surgery, Yale New Haven Medical School, New Haven, Connecticut, USA

\section{Introduction}

The recent development of transcatheter closure technique with Atrial Septal Occluder device provides an alternative therapeutic choice to surgery. However large secundum defects with limited septal margins or fenestrated septum are less suitable for percutaneous closure. We have been operating on these patients with a minimally invasive ASD occlusion technique which is combined the characteristic of both standard surgical ASD closure and intervention closure. We report our initial experience and mid term outcome with Transthoracic Minimally Invasive ASD Occlusion

Method: The aim was to evaluate the safety and efficacy of ASD Occlusion by a transthoracic minimally invasive pathway. Nine patients with secundum atrial septal defects were selected for this procedure. We used a new product which including a unique delivery system through a transthoracic pathway.

Result: There were 11 men and 14 women with a mean age of 26.4(9.6) years undergone this procedure from 2010 through 2018. All procedures were successful and there were no conversions to median sternotomy. Mean (SD) ASD size was $28.3 \hat{\mathrm{A}} \pm 5.0 \mathrm{~mm}$, including 2 cases for limited rim and one case for fenestrated septal. Mean (SD) procedure time were $54 \hat{\mathrm{A}} \pm 24$ minutes, Mean (SD) postoperative hospital stay was 3 (1) days. Mean follow-up reached 20.6 months (median 18, range 14-30 months). Follow up echocardiographic examination found a very small residual shunt in only one patient. The technique has no operative mortality so far. Conclusions: The presented technique provides an alternative procedure for the correction of large secundum ASD, limited rim ASD or fenestrated septum

\section{STUDY OF RISK OF MAJOR STROKE ASSOCIATED WITH IMPLANTATION OF NEWER LVAD}

Dr Sanjay Kumar,Dr Vladimir Shumaster 1 Department of Cardiothoracic Surgery, Institute of Medical Sciences, Banaras Hindu University, Varanasi, India; 2 Division of Cardiac Surgery, Yale New Haven Medical School, New Haven, Connecticut, USA

Background:Stroke remains a significant complication of left ventricular assist device (LVAD) therapy. We conducted a retrospective study evaluating patients undergoing first-time HeartWare HVAD (Medtronic Inc) or HeartMate 3 (Abbott Inc) implantation.

Methods: We studied database for all patients who received newer generation centrifugal flow LVAD between March 2010-March 2019.The primary endpoint was stroke-free survival. Stroke was defined as new neurological deficits persisting \&gt;24â€\%ohours with corresponding radiographic evidence. We excluded patients who received preoperative ECMO. Risk factors evaluated included demographics, medical comorbidities, heart failure etiology, LVADindication, INTERMACS profile, and device type. Univariate predictors and variables clinically suspected to raise stroke risk were entered in a multivariate hazard regression model. Results: A total of 109 HVAD and 51 HM3 patients were analyzed. Median follow up (until death, censoring for transplant/ LVADremoval, or end of follow up) was 1.2 years in HVAD patients and 1.4 years in HM3 ,patients. Stroke occurred in 24 HVAD patients (15 ischemic, 9 hemorrhagic) and $6 \mathrm{HM} 3$ patients (4 ischemic, 2 hemorrhagic). One-year stroke-free survival was $76.8 \%$ for HVAD and $84.3 \%$ for HM3. Thirty-day mortality following stroke was $41.7 \%$ for HVAD and $66.7 \%$ for HM3; $54.2 \%$ of HVAD strokes were disabling compared to $83.3 \%$ of HM3 strokes. Age, LVADindication, and device type were associated (Pâ€\%o\&lt;â€\%o.12) with stroke on univariate analysis. On multivariate analysis, the HVAD was associated with significantly higher stroke risk (hazard ratio, $2.57 ; 95 \%$ confidence interval, 1.02-6.44; Pâ€\%o=â€\%o.045).

Conclusions: HVAD is associated with higher stroke risk. However, stroke associated with HM3 appeared more disabling. Different LVADmodels were associated with significantly different stroke risks.

\section{CARDIAC HYDATIDOSIS SURGICAL MANAGEMENT - 2 CASE REPORTS \\ DR Jignesh Kothari, DR Ishan Gohil UNMICRC}

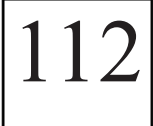

Hydatid Cyst results from infection from Ecchinococcus Granulosus and its cardiac involvement is rare even in endemic countries like India. Its diagnosis is so rare that Cardiac CT is vital for its management. Moreover surgical management of Cardiac cysts is imminent but it poses pre op , per op and post op complications in view of the antigenic nature of the hydatid cyst fluid. Here in we present two such cases of $39 \mathrm{y}$ and $40 \mathrm{y}$ females with large pericardial cysts which were managed successfully with surgical intervention without any residual cysts or complications.

\section{MULTIPLE HYDATID CYST OF LUNG: A CASE REPORT AND REVIEW OF LITERATURE}

Dr. Ansul Kumar

Department of CTVS, Rajendra Institute of

Medical Sciences, Ranchi/Jharkhand

Hydatid infestation of the lung can be primary or secondary. In three of four cases the cyst is a single one. Hydatidosis of a different location, particularly the liver, may be associated. The period of initial growth of primary hydatidosis is frequently asymptomatic. Bronchial fistulization is an important event in the evolution of the cyst. Intrapleural rupture constitutes a rare eventuality,but it is often as characteristic as it is severe. Secondary, metastatic hydatidosis, due to breaking of a primary visceral cyst in a vein or heart, is rare. A special form is so-called multiple malignant pulmonary hydatidosis, which causes progressive respiratory deficiency and right ventricular failure. A 35-year old female presented with complaint of progressive dyspnea and heaviness over right chest since six months. She was evaluated clinically and radiologically suggesting multiple hydatid cyst of right lung and single hydatid cyst of left lung. Based on findings decision to operate on right lung was taken 
in view of large size of cysts and risk of rupture of same. A right posterolateral thoracotomy was done. A total of nine hydatid cysts were evacuated from right lung. Despite the low mortality and the limited recurrence rate, it is necessary to remember the invading character of pulmonary hydatid disease, with therapy aimed at surgery and drugs.

\section{A CASE OF EXTRAPULMONARY KOCH'S ,WITH PERICARDIUM THE ONLY SITE ASSOCIATED WITH CORONARY ARTERY DISEASE. \\ Zubair Rashid \\ KIMS Hospital}

53 years old gentleman presented with DOE III for 6 months and low grade fever off andon. Hewas hospitalized in a local hospital in which he was diagnosed with Pulmonary Kochâ€ $€^{\mathrm{TM}_{\mathrm{S}}}$ and was started withAKT. Hrecompleted 2 months of intensive phase \&amp; 4 months of continuous phase was running while he again developed DOEIII.hewas again hospitalized and investigations revealed severe constrictive pericarditis, left empyemathoracis and coronary angiogram showed asinlevessel coronary artery disease. He was operated andpericardectomy was done, with left decortication \&amp; his coronaries were managed medically.

\section{CHONDROSARCOMA OF CHEST WALL: SURGICAL RESECTION AND RECONSTRUCTION USING POLYPROPYLENE MESH AND BONE CEMENT}

Anuj Sangal, Arvind Goyal, Soumyadipta

Mishra, Subhash Sinha

Max Superspecialty Hospital

Introduction-Primary malignant tumors of the chest wall are uncommon. Chondrosarcoma is the most common malignancy of the sternum. Chondrosarcomas present a difficult clinical problem due to its high resistance to conventional chemotherapy and radiotherapy. Hence, complete surgical resection has been the cornerstone for treatment. Patients with chondrosarcomas generally have a good prognosis with surgical modality. Methods-We present the case of a 35-year-old woman with upper-sternal mass. Thorax computed tomography revealed a welllineated, hypodense and round mass, which highly suggested the sarcoma of the chest wall. The tumor involved $1 / 3$ proximal part of the corpus sterni and it was largely located on ventral table of sternum and no external chest wall deformity was appreciable. In order to obtain disease-free surgical margins, $1 / 3$ proximal part of the sternum with costochondral junctions was resected and reconstruction of anterior chest wall was performed with prolene mesh and bone cement. Results-The postoperative course was uneventful. The prolene mesh with bone cement provided the essential rigidity and minimal elasticity over the surgical wound.

\section{DIRECT CLOSURE OF SECUNDUM TYPE ATRIAL SEPTAL DEFECT \\ Prof. James Thomas, Dr. Abhay Jain, Dr. Abiah Jacob \\ D.Y. Patil University Hospital, Administration \\ 3rd Floor, Sector 5, Nerul, Navi Mumbai 400706.}

Aim of this study was to look at direct closure of secundum ASDs using single suture material even in large defects. Atrial Septal Defects are usualy closed with a Decron or Pericardial patch. Use of autologus right atrial flap for closure of ASD has been described by Darian OT, Kunt AS, et al from
Sanlunif, Turkey. Laza Velicke et al used one way flap patch with slit in pulmonary hypertensive patients. Choudhary SK, Talwar S, talked about unidirectional Valved patch in ASDs. Folded double layer durable pericardial patch has been used by MEHMET et al. Trans catheter closures of ASD has been an accepted alternative in Ostium secundum defects. Material and method .82 patients underwent ASD closure from 2007 to 2019 in the unit, 56 patients with secundum type with defect size varying from 1.5 to $4 \mathrm{~cm}$ were closed directly using single suture of 3 ' $\mathrm{O}$ ' or 4 ' $\mathrm{O}$ ' proline sutures. The age of patients varied from 19 months to 62 years females were $56 \%$. Clinical and Echocardiographic assessment was done immediately after and on OPD follow up. Trivial to mild leak was seen only less than $4 \%$ patients. Pulmonary hypertension seem regressed in most patients. There were only 2 patients who expired on the fourth day for other reasons. ASD closures with patch of Decron or pericardial have also shown to have leaks at some point of some corner of the patch. Careful direct closure of ASDs using single suture is faster and safer in patients.

\section{INSTITUTIONAL REVIEW OF} NEUROENDOCRINE LUNG TUMORS

Grancy Vijayraj Monteiro,

Dr. Sreekar Balasundaram, Dr. Suresh Kumar

St. John's Medical College Hospital

Aim- To describe neuroendocrine tumors of the lung- our experience with various clinical presentations, gross morphology,pathological profile ,surgical approaches and outcomes.

Materials and method-It was a retrospective study of 10 years. We had 20 patients with euroendocrine tumors of the lung, out of which 5 were operated and 15 were deemed inoperable. We used hospital files, out patient records, pathology reports and radio-logical studies for data collection. Patients were followed up for a period of 6 months to 1 year Conclusion- Neuroendocrine tumours are a heterogeneous family of neoplasms ranging from quite indolent lesions with long-term life expectancy to extremely aggressive tumors with very poor prognosis. Due to various different presentations and combination of tumors, it is a difficult entity to treat and manage. Only few studies exist concerning these tumors and further research is needed to clarify the role of potential targeted therapies and different surgical approaches and to improve the differential diagnosis of these diverse and clinically challenging tumors.

\section{LIMITATIONS OF TRANSIT TIME FLOW MEASUREMENT IN ASSESSING INFERO LATERAL GRAFTS IN CORNARY ARTERY BYPASS SURGERY \\ Soumyajit Ghosh, Sandip Sardar, Kunal Sarkar \\ Medica Superspecialty Hospital, Kolkata}

Objective-Transit time flow measurement has been an accepted tool of assessing graft patency in CABG. Recent guidelines had recommended the method with IIa indication with level of evidence $: b$. In spite of its utility, this method has low sensitivity and moderate specificity. This arises from the ambiguity in measuring the prescribed parameters in situations of reduced mean perfusion pressure, which occurs as and when the heart is displaced. This is more prevalent in the infero- lateral aspects of the heart. Flow measurements nearer the proximal anastomoses are prone to give fallacious results. The study investigated the certainty with which inferolateral grafts could be assessed.

Method :- 367 grafts in 120 patients were assessed with transit time flow measurement. 94 patients had off pump CABG and 34 had on pump CABG. The basic parameters adopted for graft assessment were as follows :-No. of patients (TVD) - 120Grafts performed - 367(mean 3.02) Coronaries Grafted LAD 120 Diagnonal 26 Obtuse marginal 115 (OM178, OM2-37) Posterior Descending 106 Grafts in the front of the heart (LAD DG RCA) - 185 Grafts in inferolateral aspect (OM PDCx PDRCA) 
- 182 If the mean arterial pressure is reduced significantly, during probe placement near the distal anastomoses, then the consequent graft flow , pulstality index and diastolic flow data were considered to be unreliable. Results :-No. of patients (TVD) - 120 Grafts performed - 367(mean 3.02) Coronaries Grafted LAD 120 Diagnonal 26 Obtuse marginal 115 (OM178, OM2-37) Posterior Descending 106 Grafts in the front of the heart (LAD DG RCA) - 185 Grafts in inferolateral aspect (OM PDCx PDRCA) - 182 LIMA SVG GRAFT Flow 20ml/minute 30ml/minute Pulsatality Index \&1t;5.0 \&lt;5.0 \% Diastolic Flow 60\% 60\% (RCA 30\%) Conclusive (Unsatisfactory Grafts) Inconclusive $\mathrm{p}$ Value Anterior grafts (185) 160 (18/11.5\%) 25 Inferolateral (182) 100(13/13\%) 82 p\&lt;0.001 Conclusion:-Adequate mean arterial pressure in a pulsatile form, is a mandatory requirement for transit time flow assessment. The flow probe needs to be positioned as close to the distal anastomoses as possible for an accurate distal flow assessment. Its low sensitivity is due to the limitation in assessing inferolateral grafts which entail significant displacement to place the probe close to the distal ananstomosis. Most of the assumptions of accuracy of graft assessment by transit time flow measurement has been based on data derived from measuring grafts in the anterior aspect , which did not require significant displacement. We recommend improvement in probe design, preferably based on a flexible or cable mounted probe that will minimize significant displacement, and make a more accurate flow estimation possible.

\section{EXTENSIVE PULMONARY AND INTRA PERICARDIAL PRIMARY THORACIC HYDATID CYST \\ Bharath Bhogavalli, DR. R.V Kumar}

Nizams Institute of medical sciences

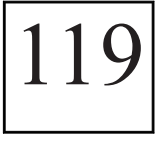

Hydatid cysts are usually located in the liver, lung, and brain. Extensive pulmonary and pericardial hydatid disease is very rare, incidence is 0.1 $0.5 \%$ and usually managed as a staged procedure depending on cyst location and number. We report a case of 19 years old female who presented with complaints of chest pain for 3 years, acute onset of shortness of breath and dysphagia for both solids and liquids in 15 days. She was a thin built poorly nourished individual. Chest radio graph and CT chest revealed multiple intrathoracic cystic lesions. Median sternotomy was done, and cystectomy with curettage was done. Histology confirmed multiple daughter cysts suggesting hydatidosis. We describe this case due its rarity of location in the intrapericardial and bilateral pleurae as a primary site for which we could surgically treat the patient by excising the daughter cysts in the both pleurae and pericardium in a single setting via median sternotomy, thereby eliminating the need for multiple surgeries and use of extensive clam shell or multiple incisions over the chest wall.

\section{TAKEDOWN OF CAVOPULMONARY ( GLENN) SHUNT USING RIGHT ATRIAL FLAP: CASE REPORT \\ Pratik Pandey, Debasis Das, Nilanjan Dutta, Shubhodip Das, Manish Sharma, Rangan Koley, Amitabh Chatarjee \\ Department of cardiac surgery, cardiac \\ anesthesia, pediatric cardiology- Narayana \\ Superspeciality Hospital Howrah Kolkata}

Background: With advances in surgical techniques, many patients who are initially palliated by a cavopulmonary shunt ( Glenn), now under go biventricular repair along with take down of cavoplumonary shunt. The surgical decisions for such situations are often complex but reestablishing continuity between the right atrium ( RA) and superior vena cava( SVC) is a common step in all such surgeries. Several authors have reported techniques for bidirectional Glenn takedown, but most are direct re-anastomosis between the SVC and RA.
Aims/ Objectives; To develop a simple technique of re-establishment of SVC to RA connectivity in biventricular conversion post BD glenn

Material and Method: We report a technique ( Described by Bairdet al) whereby a flap of right atrial tissue is used to re-establish tension-free SVC-RA continuity while preserving sinus node function. We performed it by taking SVC off pulmonary artery and attaching it with an RA flap forming posterior wall and bovine pericardium forming anterolateral wall.

Results: We have used this technique in two cases of Double outlet Right ventricle with VSD, Pulmonary stenosis who had undergone previous cavopulmonary shunt. Both the patients recovered uneventfully without any gradient between SVC and RA and have remained in sinus rhythm. Conclusions: RA flap technique is simple, reproducible way taking down a previous cavopulmonary shunt to create a tension free anastomosis. The use of RA flap as the posterior wall is the core of this technique which also has growth potential.

\section{MISSION MICS - MAKING IT HAPPEN AND MAXIMIZING RESULTS \\ Vinod Kumar Manikala, Isha P Khonde \\ Sri Narayani Hospital and Research Centre, Vellore}

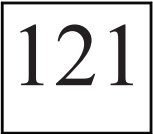

Aims and objectives: Minimally invasive cardiac surgery (MICS), although well-established in literature and in practice at advanced centres, continues to elude the reach of small volume centres and the general public. There are many apparent limitations to MICS cost, time, lack of expertise and proper equipment which, in reality, only limit the benefits we can offer to deserving patients. We aim to present our experience in developing the MICS program at our small volume cardiothoracic surgery unit at Sri Narayani Hospital and Research Centre, Vellore, Tamil $\mathrm{Nadu}$; and to share our vision for the future.

Materials and methods: We discuss our two-year experience and strategies in setting up an MICS program, review the basic training and instrumentation necessary, approach to proper patient and instrument selection, administrative and technical difficulties, and our on-going efforts to minimize costs and re-invent ourselves as a team.

Results: About 120 patients were operated in our MICS program over the past two years. We present details of the disease spectrum and variety of surgeries we were able to perform with our existing infrastructure. Valuable learning experience and guidance received from various MICS centers across the country is highlighted.

Conclusions: A simple and reproducible MICS set-up is a worthy goal to pursue. The well-known advantages of the MICS approach far outweigh the troubles caused by obstacles that can be overcome through persistence and consistency. We hope our experience will inspire others to pursue this minimally accessed path in their journey towards excellence in cardiothoracic surgery.

\section{REDO CABG- SHORT TERM AND MID TERM RESULTS FROM A DEDICATED CORONARY CENTRE} Jeevesh John Thomas,

Bhaskar Ranganathan, Simon Philipose,

Shylesh kumar, Jose Chacko Periapuram

Lisie Heart Institute, Lisie Hospital, Kaloor, Kochi, Kerala

Aims and objectives- Redo coronary surgery is considered one of the most challenging cardiac surgeries in the adult. The widespread use of internal mammary artery and other arterial conduits has reduced the number of patients needing redo CABG worldwide but in India, there is a significant population of patients requiring this because of the diffusely diseased coronary targets and the high prevalence of diabetes. Safe reentry to the chest, preservation of patent conduits, especially the LIMA, adequate exposure of coronary targets and safe myocardial protection are 
some of the challenges that the surgeon faces. We sought to find out the caveats of doing this surgery safely from our experience in a high volume coronary centre.

Methods- We looked into the details of patients who had CABG at our institute in the period 2013 to 2018. We did 21 redo coronary surgeries in this period and these were the patients included in this study.

Results- The mean age of patients who underwent redo CABG was 62.6. The previous $\mathrm{CABG}$ was done an average of 8.01 years before the redo. The average Euro score II at redo was 6.35 (2.13 to 17.9) and the mean ejection fraction at redo for the patients with dysfunction was 43.08 (30 to 55). 18 of our redo CABGs were done off pump. IABP (Intra Aortic Balloon Pump) support was not needed in any of these 21 patients. The average number of grafts done at redo was 2.65 (ranging from 1 to 4 ). Arterial grafts were used for 13 patients and 5 of them had total arterial grafting. GSV (Great Saphenous Vein) alone was used in 8 patients. The mortality in our series was 0 and there were no re-explorations. The mean ICU stay was 3.8 days and mean hospital stay was 7.28 days. There was no requirement of blood transfusion in 9 patients. For the remaining, the average units transfused was 1.67.

Conclusion- Redo CABG is a safe surgery in selected individuals if performed with care and diligence. Off pump is a viable and safe option with shorter ICU and hospital stay, reduced blood transfusions and possibly better myocardial protection.

\section{AN INSTITUTIONAL EXPERIENCE OF MANAGEMENT OF DEEP STERNAL WOUND INFECTION AND IMPACT OF VACUUM ASSISTED CLOSURE THERAPY \\ Dr Ravdeep Singh Sohal, Dr Amit Pushkarna \\ Military Hospital Cardio-thoracic Centre, \\ Pune , Maharashtra}

Aim: The aim of our study is to compare and analyse our experienceof Management of Deep Sternal Wound Infections with Open Dressing and Vacuum Assisted Closure Device Therapy and outcome. Methods: We analysed and compared our data ( Group I ) from 01 May 2009 to 31 May 2012 when the modality of management of DSWI was open dressings and irrigation (ie; PreVAC era) with data (Group II) from 01 May 2013 to 31 Apr 2017 when the modality of management of DSWI was changed to Vacuum Assisted Closure Device Therapy. 2228 patients underwent sternotomies from 01 May 2009 to 31 May 2012 and 2168 patients underwent sternotomies from 01 May 2013 to 31 Apr 2017. In Group I postoperative DSWI was diagnosed in 22 patients There were $14(63.63 \%)$ Males aged 45 to 81 years and $08(36.37 \%)$ Females aged 43 to 71 years. and in Group II postoperative DSWI was diagnosed in 20 patients. There were 11 (55 \%) Males, aged 39 to 74 years and 09 (45\%) Females, aged 44 to 66 years. The outcomes were investigated with KaplanMeier method, log-rank test, Student's test and Fisher's exact test. Results: In Group I - 17 (77.27 \%) patients underwent Secondary suturing with tension bands and $01(4.5 \%)$ patient required flap cover to cover the defect. The mortality was $04(18.18 \%)$ ( 03 Male and 01 Female), whereas in Group II- 16 (80\%) patients underwent Secondary suturing with tension bands and 02 (10 $\%)$ patient required flap cover to cover the defect. The mortality was $02(10 \%)$ ( 01 Male and 01 Female).

Conclusion: As compared to open dressings and irrigation, VAC therapy is safe and reliable option in Deep sternal wound infection after cardiac surgery, with excellent survival and low failure rate. VAC reduces mortality, increases sternal stability, patient comfort and can be used in any Hospital. Pre-operative Diabetes Mellitus, Renal Failure and Obesity are risk factors for mortality in patients treated with both modalities.
CORONARY CAMERAL FISTULA

DR. Madasu Sudhakara Rao,

DR. Anand Agarwal

DR. Vengal Reddy

Malla Reddy Narayana

Multispeciality Hospital,

Jeedimetla, Hyderabad

Coronary cameral fistula is a rare entity and is characterised by an abnormal communication between coronary artery and a cardiac chamber. It is usually congenital and asymptomatic in majority of patients. We present a case of 28 years old male who presented with anginal chest pain and dyspnoea on exertion for the last one week. His TMT was positive for ischaemic changes and ECG - gated contrast enhanced CT scan was done for further evaluation. CT showed a large branch from circumflex coronary artery to right atrium fistula. We have done conventional coronary angiogram also to rule out any coronary atherosclerosis as reason for chest pain and ischaemic symptoms on exercise treadmill test. The fistula was successfully closed by surgery and there was resolution of chest pain and dyspnoea. Case presentation with short video demonstration of the surgery will be presented. Key words: chest pain, CT and conventional angio, coronary cameral fistula

An Interesting case of Haemoptysis

Dr. Sreekar Balasundaram, Dr. Suresh Kumar,

Dr. Abin J

St. John's Medical College Hospital, Bangalore

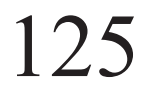

1) AIM/OBJECTIVE: To present an interesting case of Hemoptysis in a 30year old male, it's differential diagnosis and management.2) MATERIAL AND METHOD: A 30-year-old male farmer presented with a one-month history of cough and moderate hemoptysis. He had no fever, loss of weight, or past tuberculosis. His PFT was normal. Chest X-Ray showed an air-fluid level in the region above the left hemidiaphragm. The fundal shadow was not distinct. He was further evaluated with a CT Chest and an upper GI Endoscopy, which revealed Intralobar Pulmonary sequestration with aberrant arterial blood supply from the celiac trunk. Evaluation, diagnosis, differential diagnosis, pathology, and management of this rare pathology will be discussed.3) RESULT: The patientunderwent a successful Left Lower Lobectomy with ligation of the aberrant artery.4) CONCLUSION: Intralobar Pulmonary Sequestration is a rare cause of hemoptysis that needs to be evaluated meticulously for good surgical outcomes.

\section{OPEN SURGICAL RECONSTRUCTION OF THE EXTRACRANIAL INTERNAL CAROTID ARTERY ANEURYSM AT THE BASE OF THE SKULL: AN EXPERIENCE OF FIVE CASES IN A TERTIARY VASCULAR SURGERY CENTRE- GRANT MEIDCAL COLLEGE,MUMBAI,MAHARASHTRA}

Dr Suraj Wasudeo Nagre, Dr Saptrishi Paul, Dr M M Joshi Grant Medical College, Mumbai, Maharashtra

Aim: Aneurysms of the internal carotid artery (ICA) at the base of the skull are uncommon dangerous lesions whose management remains unclear. The aim of this retrospective study is to report the results of open surgical repair done in our centre in order to formulate a standardized surgical technique of ICA reconstruction with long-term results.

Materials and Methods: From 2017 to 2019, totally 5 ICA reconstructions were performed for EICA: 2 male patients and 3 female patients (mean age, 34 years). The cause was atherosclerosis ( $n=3$ patients), trauma ( $\mathrm{n}=1$ patient), and syndromic (associated with a complex 
congenital cardiac condition, $\mathrm{n}=1$ patient). The symptoms were mainly swelling and pain, in one case.

Results: All patients underwent open extracranial surgical reconstruction at the skull base with direct end to end anastomosis. The ICA was patent on the postoperative angiogram in all cases. All patients are doing well on follow up.

Conclusion: Direct end to end anastomosis for EICA can be performed safely and easily with an open surgical approach and produces durable satisfactory results.

\section{EVALUATION OF THE OUTCOMES OF THE BIDIRECTIONAL GLENN SHUNT PROCEDURE IN PATIENTS WITH CYANOTIC CONGENITAL HEART DISEASE: AN OBSERVATIONAL SINGLE CENTRE STUDY \\ DR. Ranajit Beeranna Naik, \\ DR. Chandra Prakash Srivastava, \\ DR Saify Arsiwala, DR. Ankit Mathur, \\ DR Sunil Sharma \\ Narayana Multispeciality Hospital, Jaipur}

Aim/Objective(s): Evaluation of the pre-,intra and postoperative outcomes of the Bidirectional glenn procedure as a palliative procedure in the patients with cyanotic congenital heart disease.

Material and method :We reviewed the medical records of 91 patients who underwent Bidirectional glenn operation between December 2015 and November 2019 in single centre restrospective observational study. Patient characteristics included age, weight, primary diagnosis and previous surgery. Operative technique included cardiopulmonary bypass duration, cross clamp time as well as concomitant procedure. Outcome data assessed duration of chest tube drainage, length of stay in intensive care and hospital, morbidity and moratlity during the hospital stay.

Result(s): Themedian length of intensive care and post operative hospital stay was 5 and 8 days respectively. 29 of 91 patients had postoperative complications ( $32 \%$ ). Mortality was 12 of 91 patients.

Conclusion: Bidirectional Glenn continues to be a good palliative procedure for single ventricle physiology. The outcomes are unaffected by the presence of left superior venecava. Outcomes are affected by anatomy, operative technique and cardiopulmonary bypass time.

\section{A CLINICO-PATHOLOGICAL ANALYSIS AND EXPERIENCE WITH VIDEO ASSISTED THORACOSCOPIC SURGERY IN MANAGEMENT OF MEDIASTINAL MASSES \\ S.G.K Arif, R. Basu, B Das \\ R.G. Kar Medical College Kolkata}

Objectives: The experience with Video Assisted Thoracoscopic Surgery in its management in our institution, considering size andlocation of tumor removed, operative time taken, intraoperative blood loss, post-op ICU stay,intraoperative and postoperative complications. Material \&amp; Method Study area :R.G. Kar Medical College Kolkata

Sample size \&amp;sample technique : Sample size consisted of 61 patients. Patients diagnosed to have mediastinal masses were included in this study, Including mediastinal lymphadenopathy (those having no clinically palpable cervical lymph nodes), Patients were included in this study all of them underwent either Thoracotomy or Video Assisted Thoracoscopic surgery.

Data collection technique and tools: Presenting symptoms, physical findings, and other demographic information were recorded for each patient as an interview. Using preoperative radiographic studies and intraoperative findings, the size of the tumors and their relations to vital structures were observed. The postoperative hospital stay, chest tube duration, and change of patient symptoms were recorded. Longer term outcomes included the patients activity levels at the 4-week postoperative clinic visit, resolution of symptoms, evidence of cyst or neoplastic recurrence, and other late complications.

findings: .The ages of the 61 patients ranged between 7 and 79 years, (mean $48 \hat{\mathrm{A}} \pm 18.1$ years). There were 36 male patients whose ages ranged between 7 and 79 years (mean $51 \hat{\mathrm{A}} \pm 16.9$ years) and 25 female patients whose ages ranged between 15 and 74 years (mean $43.4 \hat{\mathrm{A}} \pm 19.1$ years). Symptoms were present in 50 patients $(81.9 \%)$ out of the 61 patients and 11 patients $(18 \%)$ were asymptomatic. 17 of the symptomatic patients (34\%) had malignant disease while 33 of the symptomatic patients $(66 \%)$ had benign disease. There were 20 patients (32\%) with malignant mediastinal masses and 41 patients $(67.2 \%)$ with benign masses. The incidence of symptoms among patients with malignant mediastinal masses was $85 \%$. This is within the range of $72 \%$ to $90 \%$ of literature. The incidence of symptoms among the patients with benign disease was $80.5 \%$. This is higher than $46 \%$ to $50 \%$ quoted for the large series in the literature. Symptomatic benign disease contributes significantly to the number of symptomatic patients in our study, this may be due to small sample size of patients with malignant disease. The proportion of cases symptomatic versus asymptomatic is in accordance with previous study. 8 $(72.7 \%)$ of the 11 asymptomatic patients had benign disease and the remaining 3 patients $(27.3 \%)$ had malignant mediastinal masses. The commonest symptoms in this series were cough, dyspnoea and chest pain. These symptoms were more frequently associated with benign disease. While vein compression symptoms, haemoptysis and hoarsness were more associated with malignant mediastinal disease. The anatomical location of the tumours in our study was anterosuperior $(77 \%)$, middle mediastinal $(8 \%)$, posterior mediastinal $(15 \%)$. This pattern does not go with findings in other reports that range between $49 \%$ and $59 \%$ for anterosuperior, $18 \%$ and $25 \%$ for middle mediastinum and $23 \%$ to $29 \%$ for posterior mediastinal masses, the cause for this may be due to regional difference and small sample $(\mathrm{n}=61)$ of patients in present study. In comparison with a large collected series of 3,805 patients with primary mediastinal tumours and cysts,the incidences of neurogenic, bronchogenic, pericardial origin masses were less in present study, this again may be due to small sample of patients and regional difference in present study. VATS lesion biopsy have led to histologic diagnosis in all our patients that underwent the procedure. Perioperative and postoperative mortality was $0 \%$ for cases with VATS biopsy and resection. Furthermore, complications were noticed in $4 \%$ and $0 \%$ of our patients respectively. In comparison with thoracotomy, the mean operative time, mean blood loss,post-op ICU stay in VATS group were significantly less, in case of biopsy of mediastinal masses. In case of excision of mediastinal mass excision, the mean pleural drainage and post op ICU stay were significantly less in VATS compared to thoracotomy. The size of tumor excised was comparatively greater in thoracotomy compared to VATS. There was no significant difference in mean operative time and blood loss between the two procedures compared. In comparison with previous studies conducted, Diagnostic VATS, The mean operative time taken in present study was significantly higher than previous study ( $\mathrm{p} \& \mathrm{lt} ; 0.001$ ). The mean pleural drain was significantly lower in present study compared to previous study. The no. of cases converted from VATS to thoracotomy, and deaths occurring during procedure, were significantly higher in previous study compared to present study.(P\&1t;0.05).Post-op pleuraleffusion was significantly higher in present study compared to previous study. In case of therapeutic VATS, previous study versus present study, the mean operative time $(\mathrm{P} \& \mathrm{lt} ; 0.001)$ and mean pleural drain(P\&lt;0.01) in present study is significantly lower than previous study. The no. of cases converted from VATS to thoracotomy, during procedure, were significantly higher in previous study compared to present study.(P\&lt; 0.05). There was no significant difference found regarding to complications and deaths during the procedure. These differences in both study groups may be due to difference in surgical expertise and difference in selection of cases

Conclusions : Incidence of mediastinal masses is more common in males compared to females as per this study. Mediastinal masses arefound to 
occur most commonly among 41 to 60 years of age group, in case ofmales, and 21 to 80 years in females. Majority of patients either with benign or malignant lesions had symptoms and signs. Dyspnea, cough, chest pain were the most common symptoms resented. Thymic lesions were the most common lesions of the anterosuperior mediastinum. Lymphnode masses were the most common lesions of the middle mediastinum, andneurogenic lesions were the commonest lesions in the posterior mediastinum. Incidence of thymicorigin tumors is the highest among all mediastinal masses. Video Assisted Thoracoscopic Surgery is abetter modality compared to convential thoracotomy with respect to durationpleural drain in situ, and post-op ICU stay in case of mediastinal massexcision, along with less mean operative time required and blood loss during procedure for biopsy of mediastinal masses. Complications occurring due to VATS were significantly lower than other modalities. Histologic yield is $100 \%$ in case of diagnostic VATS

\section{MANAGEMENT OF SEVERE VALVULAR HEART DISEASE IN THIRD TRIMESTER PREGNANCY: CASE SERIES AND ALGORITHM FOR MANAGEMENT \\ Shrikant Suryawanshi, Chaitanya Raut, Prashant Mishra, Jayant Khandekar. \\ Lokmanya Tilak Municipal Medical College and \\ Sion General Hospital, Mumbai}

Aims-Valvular heart disease is the most common heart disease complicating pregnancy. As there are no specific guidelines to address severe valve during third trimester of pregnancy we present an algorithm for management of such patients.

Materials and methods-We present a series of 8 patients presenting with severe valvular heart disease for the first time in third trimester of pregnancy over the last 5 years (Jan 2012 to Dec 2017).

Results-

Seven patients had severe mitralstenosis and one had severe aortic stenosis. Of the 7 patients with mitralstenosis, 5 presented with heart failure and the other 2 had severe pulmonary hypertension, all diagnosed for the first time during third trimester. The other patient with AS was asymptomatic. Six patients did not respond to medical management and underwent MVR with LSCS after confirmation of foetal maturity. The other 2 patients were treated medically and elective LSCS at term.

Conclusion-

Stenotic lesions are generally poorly tolerated than regurgitant or mixed lesions. Women with severe AS/MS are more likely to develop HF and atrial arrhythmias and have adverse fetal outcomes such as preterm birth and low birth weight. Women presenting with severe stenotic lesions in first trimester are generally advised again continuation of pregnancy. Patients presenting in second trimester are usually managed medically. Any intervention (percutaneous valvulotomy/surgical repair or replacement) is also carried out in the second trimester. Rarely patient present for the first time in third trimester with severe stenotic lesion.

\section{MANAGING AN ASCENDING AORTA PSEUDOANEURYSM FOLLOWING AORTIC VALVE REPLACEMENT- A CASE REPORT \\ Dr. Rohit Kumar Rathi, \\ Dr. Subhendu Mahapatra \\ Institute of Post Graduate Medical Education \\ and Research, S.S.K.M. Hospital}

Introduction: Ascending aorta pseudoaneurysm is a rare, life threatening complication occurring months to years following aortic valve surgery and occurs in frequently from anastomotic dehiscence of suture lines or at cannulation sites. When a pseudoaneurysm has eroded bony structures in the chest, it becomes a challenge for the surgeon to even approach a safe sternotomy. Materials and methods. We present a case of a $35 \mathrm{yr}$ old lady presented with complaints of swelling of the anterior aspect of chest for 10 days who underwent an aortic valve replacement 5 months back. On investigations, she was diagnosed to have pseudoaneurysm of ascending aorta eroding upper part of sternum and adjoining ribs on left side. An emergency surgery was done where stenotomy was done after CPB was initiated under axillary and femoral bypass and the rent in the aorta was repaired with bovine pericardial patch. The patient recovered well without any neurological deficit. Results. The presentation describes the characteristics of the case and the surgical decision. Conclusion. Ascending aorta pseudoaneurysm is associated with high morbidity and mortality. Entry into the chest is a challenge for the surgeon when there is bony erosion and requires pre-operative planning. Cerebral perfusion should be maintained during $\mathrm{CPB}$ either through axillary or carotid cannulation. However, every case of ascending aorta pseudoaneurysm is different and should be managed accordingly.

\section{ENDOSCOPIC THORACIC SYMPATHECTOMY FOR REFRACTORY VENTRICULAR ARRHYTHMIAS: OUR EXPERIENCES. \\ Dr. Noaman K Shaikh, Dr. Arnab De, \\ Dr. Tejas Dandekar, Dr. Manish Jadhao, \\ Dr. Vaibhav Shah, Dr. Chaitanya Raut, \\ Dr. Prashant Mishra, Dr. Jayant V Khandekar \\ Lokmanya Tilak Municipal Medical College \\ and Hospital, Sion, Mumbai.}

Aim:To assess the outcomes of Endoscopic Thoracic Sympathectomy (ETS) on patients with catecholaminergic polymorphic ventricular tachycardia, refractory to pharmacologic, cardiac ablation and implantable cardiovert defibrillators. Methods: Bilateral ETSs were performed in a one-stage procedure by the same surgeon under general anesthesia and selective ventilation. Patient was placed in standard lateral thoracotomy position. All patients had an intercostal drain inserted bilaterally post sympathectomy which was removed on post-op day 1.Patients were followed upto 1 year post surgery, and were regularly evaluated clinically and by ICD interrogations. Results: A total of 8 patients diagnosed with non-sustained ventricular tachycardia underwent ETS. 1 of the 8 patients had the need for conversion of right endoscopic procedure to an open thoracotomy in view of dense adhesions in the pleura. Of the 8 patients operated, there was only 1 incidence of Hornerâ $€^{\mathrm{TM}_{\mathrm{S}}}$ syndrome. Compensatory hyperhidrosis was observed in all 8 patients but was mild in $75 \%$ of the cases. The incidence of moderate and severe compensatory hyperhidrosis was $12.5 \%$ each. Six patients showed freedom from VT and ICD shocks at an interval of 1 year post surgery. One patient had repeated ICD shocks after a 3 month convalescent period and eventually was posted for Cardiac Transplant procedure in view of structural heart defect. One patient had 2 incidences of ICD shocks in the 1 year followup period. Conclusion: ETS is a therapeutic option for ventricular arrhythmias that are refractory to beta blockade, cardiac ablation and /or ICD for the prevention of lethal arrhythmias and sudden cardiac arrest.

\section{CONTAINED RUPTURE OF EXTRACRANIAL CAROTID ARTERY ANEURYSM IN A CORONARY ARTERY DISEASE PATIENT- CASE REPORT AND REVIEW OF LITERATURE}

Manoop B, Vinu C V

Government medical college, Thiruvanathapuram

Aim - Extracranial internal carotid artery aneurysm is an uncommon yet important clinical entity, and true incidence being less than $1 \%$ of all 
carotid etiologies. We report a case of contained rupture of extracranial internal carotid artery aneurysm in a $65 \mathrm{yr}$ old patient with single vessel disease. Materials and methods- Patient underwent $\mathrm{CT}$ angiogram neck which showed well defined saccular aneurysm from Rt carotid bulb with contained hematoma lateral to it. CT angiogram brain showed intact circle of Willis with minimal narrowing of V4 segment of Rt vertebral artery. After echo study, patient was taken up for Emergency ON PUMP BEATING CABG with single ReSVG to LAD. Aneurysmal excision was done and reconstruction done by proximal CCA - distal ICA bypass with PTFE graft under low flow cardiopulmonary bypass with profound hypothermia at 18 degree celcius. Results- Postoperatively there were no focal neurological deficits. Patient required minimal inotropic supports and was extubated on POD 2. Pt was ambulated on POD 4 and was discharged on POD 10. Post operative echo showed good LV contractility and repeat angiogram showed good graft patency. Conclusion- Non traumatic ECCA is a rare entity and so is there presentation with rupture. Prompt clinical and radiological assessment and prompt decision is important to save patient's life and to prevent neurological deficits. We opted for Emergency surgical correction under cerebral protection in view of tortous arterial course with poor landing zone and unfavourable hemodynamic status.

\section{EXTRAPLEURAL HYDATID CYST}

Vipin N Nair, Abdul Rasheed

Government Medical College Trivandrum

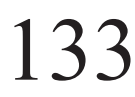

38 yr Asymptomatic female was detected with an incidental right side pleural based mass lesion on chest xray. MRI suggested nerve sheath schwanoma from D5 with erosion. On table finding of hydatid cyst with multiple daughter cyst inside. Complete excision done. Intra operative and post operative period managed cautiously without evidence of any advert events. She was dischrged on pod6. Extrapleural hydatid has been reported less than $1 \%$ worldwide.

\section{STELLATE GANGLIONECTOMY- AN UNCOMMON PRESENTATION: \\ A CASE REPORT}

Dr. Vikas, Dr. Amresh

NIMS, Punjagutta, Hyderabad

\section{INTRODUCTION:-}

The Stellate Ganglion (or cervico thoracic ganglion /inferior cervical ganglion ) is a sympathetic ganglion formed by the fusion of the inferior cervical ganglion and the first thoracic ganglion. It is located at the level of $\mathrm{C} 7$ (Cervical vertebrae ) anterior to the transverse process of $\mathrm{C} 7$, anterior to the neck of the first rib and just below the subclavianartery. Indicationsof sympathectomy :-

1) Peripheralvascular disease temporary vasodilatation development of collaterals

2) Hyperhidrosis- Permanent relief

3) Relief of pain visceral pain excision /distruction coeliac ganglion ca. Pancreas, chronic pancreatitis

CASE REPORT- A 19yrs old female, c/o- Recurrent episodes of palpitations and giddiness since $5 \mathrm{yrs}$. $\mathrm{F} / \mathrm{H} / \mathrm{O}$-sudden death of elder brother at the age of 15 yrs. O/E- CVS- S1 +,S2 + Chest- clear ,B/L AE +Lab reports- Hb-10.6 g/dl, TLC- 6000, PC- 2.3 lakhs/cumm GRBS- 98, urea- 24 , creatinine- $0.5 \mathrm{mg}$ /dlLFT- WNL ECG- long QT Syndrome Chest X ray- WNL 2D ECHO (pre op)-NO RWMA, EF-62\%, , NO PE, VEG, CLOT.TMT: Exercise for 7 mets. long QT. QT : $520 \mathrm{msec}$, QTC-480 msec. OPERTATION NOTES: PROCEDURE: Left supraclavicular skin crease incision given. Scalenes anterior cut. Stellate ganglion identified at neck of first rib. Ganglionectomy done. Romovac suction drain kept. Skin closed in layers. CONDITION ON DISCHARGE: Patient post operatively had chyle leak in the romovac drain, treated conservatively and advised fat free diet. Afebrile BP: 120/ 80 mm Hg PR: 88/minHeart : S1+, S2+ Lungs: clear, bilateral air entry + Wounds healthy

\section{INSTITUTIONAL EXPERIENCE OF \\ TRACHEAL STENOSIS}

MANAGEMENT-CASE SERIES

Dr. A.S. Mughilan, Karthikeyan B MS MCh, Marvin Manoah Baylis MS MCh,

Department of Cardiovascular Thoracic Surgery,

Madurai Medical College

Madurai Medical College, Madurai

Aims/ objectives Tracheal stenosis is one of the most common complications among the prolonged ventilated patients with or without tracheostomy. Post extubation, patient presented with late complication ofstridor, with a duration of minimum 3 months. Here we report our experience in the management of 11 tracheal stenosis patients.

Materials/ methods In our study, we prospectively followed 11 tracheal stenosis patients, who presented with stridor and managed with various modalities of management as surgical tracheal esection and anastomosis, tracheal dilatation with rigid bronchoscopy with Montgomery tube insertion.

Results Out of 11 patients, 8 were males and 3 were females. All patients had history of poison intake, which required prolonged ventilation either through endotracheal tube or tracheostomy tube and presented later with stridor following discharge. 2 patients with short segment stenosis $(2 \mathrm{~cm} 4$ cartilages) have been managed with surgical tracheal resection and anastomosis. Other 9patients who had long segment stenosis have been managed with tracheal dilation with Montgomery $\mathrm{T}$ tube insertion, which are removed after 6 months. 2 patients out of this presented with stridor following Montgomery tube removal, and those have been planned for surgical resection

\section{Conclusion}

When the stenotic segment was short, it can be effectively managed with tracheal resection and anastomosis. When it involves long segment, tracheal dilatation and Montgomery $\mathrm{T}$ tube insertion is an attractive alternate choice.

\section{COMPLICATION FOLLOWING LUNG RESECTION SURGERY AND THEIR MANAGEMENT â€" OUR EXPERIENCE IN TERTIARY CARE HOSPITAL IN WEST BENGAL} Dr. Madhur Sahay,

R. G. Kar Medical College and Hospital, Kolkata, West Bengal Complication Following

Lung Resection Surgery and their

Management our Experience in

Tertiary care

Hospital in West Bengal-DR.

Madhur Sahay, Postgraduate Trainee,

R. G. Kar Medical College and Hospital

Aim/objective 1) To study the etiology of lung resection of our setup2) Tostudy postoperative complications and its management

Material and method This is a retrospective observational study which included 480 patients who underwent lung resection surgeries for various disease conditions over a span of 9 years (2010-2018). The following information was recorded etiology, preoperative assessment (including Pulmonary Function Test, Fibro-optic Bronchoscopy, Sputum for AFB, CT Scan). Intraoperative and Postoperative events were also recorded separately for individual patients. 


\section{Result}

480patients were studied underwent lung resection surgeries whose diagnosis varied from Trauma, Bronchiectasis, Hydatid Cyst, Sequestration, Destroyed Lung, Carcinoid, Empyema, Aspergilloma, Infection (Tubercular and Non-Tubercular) and Malignancy. The patients commonly underwent Posterolateral Thoracotomies. The other techniques like B/L Thoracotomies (Right followed by left) for B/LHydatid Cyst and midline sternotomy for trauma in one instance each. Patient underwent lobectomy in 358 cases, Segmentectomy in 62, Decortication in 54, Bilobectomiesin 20 and Pneumonectomy in 6 cases. Out of which 395 cases were uncomplicated (extubated in 1-2 days and discharged within 7-10 days). 81 patients came up with complications which can be characterized as (1) Early (post operative period till discharge), (2) Late (from discharge till 6 months)(1) Early complication included Bleeding in 10 cases requiring re-exploration, Arrhythmias mainly following Pneumonectomy, Prolonged air leak in 42 patients which required prolong ICD in situ, Incentivespirometry, Fibro-optic Bronchoscopy, other symptomatic and supportive treatment. 17 patients got well, rest underwent Decortication of which 20 recovered and 5 required some sort of Thoracoplaty. 10 patients landed up with Sepsis treated with IV antibiotic out of which 6 had Bronchopleural fistula of which 2 recovered and 4 required re-exploration out of which 3 died.(2) Late complication included 8 patients who complained of Pain referred to Pain Clinic and required Intercostal Nerve Block. No patients had recurrent hemoptysis, wound complication or repeated cough.

\section{Conclusion}

Postoperative complication after lung resection is not very uncommon and is closely related to Preoperative assessment, selection of the patient, optimization of patient along with intraoperative technique and proper Postoperative care can minimize the complication

\section{DEMOGRAPHIC ANALYSIS OF DEEP STERNAL WOUND INFECTIONS AFTER CABG}

Sunay N M, Dr.Uday Jadhav, Dr.Balaji Aironi, Dr.Abhishek Joshi

KEM Hospital, Mumbai

\begin{abstract}
Aims and Objectives: 1.Studyingdemographic data of deep sternal wound infections in CABG 2.Microorganismsinvolved 3.Antibiotic strategies 4.Length of stay 5.Indices of Mortality

Material and Methods: 40 Patients who underwent CABG, and later developed deep sternal wound infections at our center between Jan 2014 and Nov 2019 were studied.

Results: Most patients were aged above 60 yrs. Higher incidence was seen in elderly diabetic patients with poor perioperative glycemiccontrol. Wound cultures often grew Staphylococcus, Klebsiella, Pseudomonas, followed by acinetobacter, candida, etc.

Conclusion:

Deep sternal wound infections in CABG is associated with high morbidity and mortality. However, it is a preventable condition with proper management techniques.
\end{abstract}

\section{WHATS THE BEST STRATEGY FOR DIFFUSE MULTI VESSEL DIEASE IN ELDERLY HAVING CHRONIC STABLE ANGINA}

Dr. Rakesh Kumar, Dr Rani Singh

Rajendra Institute of Medical Sciences Ranchi

Author- Dr Rakesh KumarAddress-Assistant

ProfessorDepartment of CTVSRIMS RanchiPhone

no.-7903400638Email-drrakeshc0@gmail.com,

Presenting authorDr Rakesh KumarCo authorDr Rani Singh
Aim-To know what should be done to group of elderly diffusely multivessel disease having chronic stable angina.

Material and methods-Last two years patients(50) who is above 70 years of age are included in the study. They presented to us with chest pain and when angiography reviewed they found to have difuse multivessel disease. All of the patient suggested PET scan for myocardial viability and those having heibernating myocardium were optimised with medical therapy and spirometry and underwent coronary artery bypass surgery. Results-The outcome of surgery was good in the selected group and $90 \%$ of them have got relief of symptoms. Conclusion-In elderly having diffuse multivessel disease with chronic persistent angina, the further evaluation is required for myocardial viability.

\section{MALIGNANT EPITHELOID TUMOR OF ANTERIOR MITRAL LEAFLET ; A RARE AND UNUSUAL PRESENTATION ; AN INTERESTING CASE REPORT}

Manpreet Singh Salooja, Ajeet Bana, Bishal Gautam, Ashish Madkaiker, Sandeep Tirkey, Tania

Eternal Hospitals, Jaipur, Rajasthan

Aim/ Objectives Malignant primary cardiac tumors have dismal prognosis, since they tend to infiltrate the myocadium rapidly, cause obstruction of the cardiac chambers and produce metastases. Management of choice in sarcomas is surgical resection, with for results and high rates of relapse. Chemotherapy is used as an adjuvant to help decrease tumor size and facilitate surgical resection, or cases of nonoperable or metastatic sarcomas. In spite of the aforementioned therapeutic attempts the survival rate in cases of sarcomas does not usually exceed one year. In cardiac Iymphomas, systemic chemotherapy with or without radiotherapy is usually undertaken. The stated duration of survival from the time of diagnosis varies from 7 months to a maximum of 2 years. On long-term follow-up, the majority of patients die of distant metastases. Material and methodA 63 years old female presented with history of PTCA of RCA in 2011 with history of gradual worsening dysponea on exertion. Diagnosed in ECHO as large hyperechogenic heterogenous mobile mass, attached to AML, ?nature. TEE confirmed the diagnosis. Tumor was excised. Biopsy reported high grade malignant epitheloid neoplasm with rhabdomyoblast like differentiation. Patient was discharged on post op day 7 and is doing well in follow-up and reffered to oncology for further management. Results Patient was discharged on post op day 7 and is doing well in follow-up and reffered to oncology for further management. Conclusion A high level of clinical suspicion is needed for making the diagnosis. Patients with cardiac tumors should undergo surgery in a timely fashion in a specialized center. Surgical management is undertaken in most of the cases for curative purposes, symptomatic relief and establishment of histopathological diagnosis. Adjunct therapies form a part of management plan in malignant cases.

\section{COMPLEX CASE OF AORTIC ARCH ANEURYSM WITH LEFT COMMON CAROTID ARTERY OCCLUSION \\ Jayaprakash, Nandha kumar \\ Institute of Cardiothoracic surgery, \\ Madras Medical College}

\section{Abstract}

Aortic aneurysm has an estimated incidence of 5-10per million. According to its location it is classified into aortic root or ascending aortic aneurysm, most common (â\% $\% 00 \%$ ), followed by descending aorta ( $\hat{a} \%$ $\left.{ }^{3} 35 \%\right)$ and aortic $\operatorname{arch}(\& 1 t ; 10 \%)$ and is least common. We report this rare case of a 47 hypertensive male presented with backache and on evaluation found to have combination of saccularaneurysm of Aortic arch and 
Descendingaorta with complete occlusion of left common carotid artery and was successfully managed with surgery. Keywords: Aortic archaneurysm, Tearing chest pain, hypothermic circulatory arrest Case report: 47 hypertensive male presented with back ache for 1 month. On evaluation with CT aortogram, two saccular aneurysm in arch of aorta and descending aorta and left common artery occlusion identified. After thorough evaluation he underwent Aortic archaneurysmal sac excision with aneurysmal repair using Dacron graft with Carrol's patch under deep hypothermic circulatory arrest and antegrade cerebral perfusion successfully. Post operatively, he was extubated next day and managed with multiple inotopes and other supportives. He improved well with no neurological deficits with good hemodynamics.

Conclusion:

Open aortic arch surgery for aneurysmal disease is a complex procedure with a high peri-operative risk of morbidity and mortality as it requires arresting circulation to allow for a bloodless field while at the same time ensuring the safety of the central nervous system. We report this case for its rarity and successful management of aortic arch aneurysm with left carotid occlusion ensuring cerebral perfusion intraoperatively with no neurological deficits.

\section{OUTCOME OF RHEUMATIC MITRAL VALVE REPAIR - SINGLE CENTRE STUDY OF 384 PATIENTS}

Lakshmi Kumari Sankhyan, Santosh Kumar Pandey, Sujoy Chatterjee, Nikhil Bansal, Rajarshi Ghosh,

Hemant Kumar Nayak, Sudipta bhattachariya,

Saurabhi Das, Satyajit Bose, Srirup chatterjee

The Mission Hospital, Durgapur, West Bengal, India

Aim: To study the outcome of mitral valve repair in patients with Rheumatic heart disease.

Material and methods: From January 2011 to November 2019,384 consecutive cases of rheumatic heart disease undergoing mitral valve repair were studied. Mean age was $15.64 \hat{\mathrm{A}} \pm 7.05$ years (range $6-49$ years). Two hundred and forty five patients $(63.8 \%)$ were female. Two hundred and ninety five patients $(76.8 \%)$ were in New York Heart Association functional class III or IV. Mitral valve repair procedures included ring annuloplasty in all patients, commissurotomy $(\mathrm{n}=151)$, cuspal thinning $(n=38)$, cuspal augmentation $(n=58)$, chordal release $(n=321)$, chordal transfer $(n=29)$,chordal shortening $(n=25)$ and papillary muscle splitting $(\mathrm{n}=111)$. Additional procedures included tricuspid valve repair $(\mathrm{n}=56)$, aortic valve repair $(n=42)$, combined aortic and tricuspid valve repair $(n=15)$, atrial septal defect closure $(n=20)$ and aortic valve replacement $(\mathrm{n}=11)$.

Results: There were 3 early deaths $(0.7 \%)$. Median follow up was31 months (mean $31.78 \hat{A} \pm 20.92$ months, range 1- 96 months). At follow up, 266 patients $(82.1 \%)$ had trivial to mild mitral regurgitation. Twelve patients $(3.12 \%)$ underwent reoperation for valve dysfunction. There were 11 late deaths $(2.86 \%)$. Overall survival was88.79 $\hat{\mathrm{A}} \pm 2.14 \%$.

Conclusion: The complexity of Rheumatic valvular disease makes it difficult to choose an appropriate procedure for any given patient. Mitralvalve repair in rheumatic heart disease is a feasible option with acceptable outcomes.

\section{ANOMALOUS ORIGIN OF LEFT ANTERIOR DESCENDING ARTERY FROM THE PULMONARY ARTERY - A RARE ENTITY}

Dr Varun Sisodia, Dr S.S Lohchab, MCH Resident PGIMS RHOTAK

ABSTRACT-CASE REPORT Introduction Anomalous origin of left coronary artery from the pulmonary artery (ALCAPA syndrome) is arare congenital coronary artery anomaly with incidence of 1 in 4243 live births " $0.023 \%$.Anomalous origin of left anterior descending artery from the pulmonary arteryis even rarer. These anomalies are one of the most common cardiovascular causes of sudden death in young patients. It develops when the babyâ $€^{\mathrm{TM}_{\mathrm{S}}}$ heart is developing, early in the pregnancy. Case Report Wepresent these rare findings in a young 20 year old female patient who presented with chest pain, Angina and generalized weakness since 3 years. Her ECG showed sinus rhythm and T wave inversion over lateral leads. ECHO was negative for ischemic changes and CT coronary angiogram showed aberrant origin of left anterior descending artery from main pulmonary artery (pictures attached). Result CABG on pump with ligation of left anterior descending artery anomalous origin was done. LIMA to LAD was performed. Postoperative CT angiography as well as catheter angiography showed disappearance of collaterals from RCA to LAD and patent LIMA. After regular follow up there was improvement in general well being of the patient with disappearance of symptoms.

\section{A RARE CASE REPORT OF REDO SURGERY FOR EARLY BIOPROSTHETIC VALVE THROMBOSIS}

Dr Rahul Bhushan, Dr (Prof) Vijay Grover,

Dr (Prof) Vijay K Gupta, Dr (Prof) Narender S Jhajhria

ABVIMS and RML Hospital, New Delhi

\section{Background}

Bioprosthetic valve thrombosis was previously considered as an extremelyrare complication which has not been systemically recognized and understood. Furthermore, Incidence of thrombosis within 3 months of AVR is an extreamly rare finding.

Case presentation

Herein, we present an unusual case of a patient manifesting with acute heart failure, secondary to thrombus formation in an aortic bioprosthesis which was implanted merely 3 months back by us. Patient was taken up for surgery and Redo AVR was done after explanting the previous valve. Conclusion

Our study reviews predisposing factors, typical echocardiographic features and treatment for bioprosthetic valve thrombosis. A combination of clinical and echocardiographic features can help for diagnosis preoperatively. In some certain circumstances, reoperation can be avoided if anticoagulant therapy works but surgical explantation and reimplantation of new valve was ideal therapeutic approach.

AUTO PERICARDIAL MITRAL RECONSTRUCTION- HOW I DO IT

Prasanna Simha Mohan Rao, Rakesh Naik Lachma, Prabhu Manohar, Jeeva Vijayan,Kaushik Ramachandran Sri Jayadeva Institute of Cardiovascular Sciences and Research

We describe our technique of autopericardial reconstruction of the mitral valve . Our method is designed to standardize every step to make the operation reproducible. The video shows the various steps of preparation of pericardium, preparation of the valve for implantation, implantation technique, construction of the subvalvar apparatus and testing of the valve. Every step has been standardized to allow reproducibility. This can be a boon to younger rheumatics who face rapid degeneration of bioprosthetic valves and have to face problems of anticoagulation. We were inspired to do this procedure as we found that 10 patients who had undergone a modified Deac procedure between 2008-2009 had no degeneration over a period of 10 years. The current modification builds on the original Deac procedure to allow better valve hemodynamics and mimics the natural mitral valveanatomy as far as possible. 
TRICUSPID VALVE DEAC PROCEDURE FOR IRREPARIABLE TRICUSPID VALVE DISEASE HOW I DO IT.

Rakesh Naik Lachma, Prasanna Simha Mohan Rao

Sri Jayadeva Institute of Cardiovascular Sciences and Research

The video describes creation of an autopericardial valve based on the frustum of a cone as described by Deac et al for the mitral valve and adapted for the tricuspid valve. The video shows 4 such cases were the tricuspid valve was not repairable and required replacement. 3 of the cases were for extensive endocarditis and one case was an unrecognized otherwise asymptomatic carcinoid syndrome which was suspected when the valve was inspected on table. which was confirmed on subsequent investigations. The video describes the surgical technique and testing of the valve.. The Deac procedure applied to the tricuspid valve allows treatment of extensive endocarditis with autologous tissues with minimal foreign material and is a useful technique in the armamentarium of the surgeon.

\section{VATS DIAPHRAGMATIC PLICATION IN A CASE OF RIGHT DIAPHRAGMATIC PALSY \\ Dr G Siva Prasad Goud, Dr Balasubramoniam Yashoda Hospital}

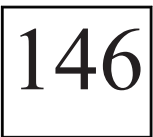

Objective: Diaphragmatic plication is performed for paralysis oreventration of the diaphragm which can result in breathing difficulties. Surgical plication to stabilize the diaphragm is needed to prevent paradoxical breathing.

Material \& Method:

We present a case of 32 years male known case of poliomyelitis presented with complaints of dyspnea was evaluated and diagnosed as right diaphragmatic palsy.

Discussion :

Diaphragmatic paralysis is typically due to damage to the phrenic nerve or abnormalities that affect the neuromuscular axis between the cervical spinal cord and the diaphragm, eventration is most commonly congenital. Diaphragmaticplication is intended to decrease lung compression, stabilise the thoracic cage and mediastinum, and strengthen the respiratory action of intercostal and abdominal muscles. More effective diaphragmatic recruitment occurs afterplication leading to increased diaphragmatic strength and maximal voluntary ventilation. Studies have shown objective evidence that all lung volumes with the exception of FRC are improved following plication. In Diaphragmatic plication the weakened area of the diaphragm is grasped with a clamp and elevated to determine the location of suture line. Layers of sutures reinforced with Teflon pads, are sewn in rows at the edge of the diaphragm along the patient's ribcage encircling the inside of their chest wall. After plication, the height of diaphragm is lowered by one or two ribs, helping the lungs inflate properly and prevent abdominal contents from pushing into the chest. The goal is to improve dyspnea by reducing dysfunctional diaphragm excursion duringinspiration; hence, operative intervention is indicated exclusively for symptomatic patients. An elevated hemidiaphragm per se does not warrant surgery in the absence of significant dyspnea. Relative contraindications include morbid obesity and certain neuromuscular disorders.

Conclusions:

Diaphragm plication is only indicated for symptomatic patients with diaphragmatic paralysis or eventration. A variety of open and minimally invasive transthoracic and transabdominal techniques have been described
ASD REPAIR WITH MVR VIA RIGHT

MINI-THORACOTOMY APPROACH WITH CONVENTIONAL CENTRAL

CANNULATION: CASE REPORT

Dr Mohit Sharma, Dr Anil Sharma,

Dr. Sunil Dixit, Dr. Dhruv Sharma,

Dr. K.K.Mahawar

SMS Medical College, Jaipur

Introduction- At present thoracotomy with femoro-femoral bypass is established approach for minimally invasive open heart surgeries but thoracotomy with conventional cannulation is yet to be established. A20-year-old young female referred to our department with ASD and severe MS. We performed intra-cardiac repair of ASD with pericardial patch and MVR through right atrium by right mini-thoracotomy approach with central cannulation (like sternotomy) without femoro-femoralbypass without any specifically designed equipment. There was no procedure related complication.

\section{SEVERE CALCIFIC PULMONARY VALVE STENOSIS WITH OSTIUM SECUNDUM ASD: A RARE CASE REPORT \\ Dr. Aparesh Sanyal, Dr. Sandeep Singh, \\ Dr. Shamsher Singh Lohchab \\ Pt. B.D. Sharma PGIMS, Rohtak}

Pulmonary valve replacement is a rare surgical procedure in case of dystrophic calcification of pulmonary valve. Usually it is done for pulmonary regurgitation after total correction procedure in Tetrology of Fallot. Bioprosthetic valve is preferred over mechanical valve during replacement, asbeing a low pressure area in case of mechanical valve even with oralanticoagulants there can be thrombo-embolic complications. Isolated pulmonary valve replacement for severe calcific pulmonary stenosis in itself is not a common procedure. In thispaper we report a case of Ostium secundum atrial septal defect with calcificpulmonary valve stenosis in a middle aged male patient. Patient was admitted with diagnosis of ostium secundum atrial septal defect and was taken up for surgery for atrial septal defect. Intra operatively while palpating the pulmonary artery for pulmonary artery pressures, a hard mass was found in the main pulmonary artery origin. Hence pulmonary arteriotomy performed and pulmonary valve was examined, which showed severe calcific stenosis. Therefore patient underwent pulmonary valve replacement with a bioprosthetic valve, atrial septal defect closure with glutaraldehyde fixed autologous pericardial patch. Patient was given oral anticoagulants for 3 months and is now on low dose aspirin only. After 4 months of follow up patient is asymptomatic. Although pulmonary valve replacement is similar to aortic valve replacement, but pulmonary valve replacement is simpler than aortic valve replacement, as there are no coronary ostia and conduction bundle along pulmonary annulus. So there are no feared complications of coronary ostia occlusion and conduction bundle entrapment. To this date this is the fifth case to be reported as per literature review.

\section{MANAGEMENT AND OVERVIEW OF POSTOPERATIVE CHYLOTHORAX AFTER CONGENITAL CARDIOTHORACIC SURGERY}

Dr Dhananjay Bansal

ABVIMS \& Dr. RML Hospital, New Delhi

Aim:

We intend to determine the incidence, risk factors, and outcomes for chylothorax in patients undergoing cardiothoracic surgery, with special emphasis on its management. 


\section{Methods:}

Total of 24 patients with postoperative chylothorax, operated from September 2015 to December 2018, were included in the study. Medical records were maintained, including daily records of drainage volumes and management.

\section{Results:}

From September 2015 to December 2018, there were 24 cases of chylothorax in 600 congenital cardiothoracic surgeriesâ $€$ "an incidence of $4.0 \%$. The overall mortality rate was similar, but cases had longer postoperative hospital stays (median, 22 versus 8 days). The incidence of chylothorax was significantly higher with congenital cardiac procedures. The diagnosis was made at a median of 6 days after surgery. Duration of drainage was a median of 15 days, with 11 patients draining more than 30 days. Longer duration of drainage was associated with cavopulmonary anastomosis procedure. Nutritional management included a low-fat diet, enteral feeds enriched with medium-chain triglycerides, and parenteral nutrition. Three patients were treated with octreotide, 4 with thoracic duct ligation, and 1 with pleurodesis. Octreotide was associated with a variable effect on drainage. Thoracic duct ligation reduced but did not stop drainage.

\section{Conclusions}

Chylothorax increases the duration of hospitalization after cardiovascular surgery in children. Early diagnosis may reduce the duration of chylothorax. Nutritional strategies remain the cornerstone for the management of postoperative chylothorax. The impact of octreotide and surgical intervention is limited when reserved for patients with severe or prolonged drainage.

\section{COR TRIATRIATUM DEXTER \\ Mrinal Patel, Dr Vishal Agrawal, \\ Dr Amit Mishra \\ UNMICRC}

Cor triatriatum Dexter (CTD) is an extremely rare congenital cardiac anomaly in which there is persistence of right valve of embryonic sinus venosus which divides the right atrium into 2 parts. It needs to be differentiated from a prominent Eustachian valve. It can present as an isolated condition or in association with other cardiac defects, mostly right sided defects like stenosis or atresia of pulmonary valve, tricuspid valve anomalies and atrial Septal Defect. The clinical presentation depends on the association and on the degree of obstruction. When CTD occurs as an isolated anomaly, the presentation may vary from asymptomatic patient, cyanosis in infancy to right heart failure in adults. We report a case of CTD in an adult patient who presented with palpitations. The patient was found to be in atrial fibrillation which was managed by rate controlling drugs. echocardiography established and computed tomography confirmed the diagnosis of isolated CTD with a hugely dilated right atrium. She underwent successful resection of the membrane in right atrium along with right atrial reduction plasty after which the patient reverted to sinus rhythm.

\section{CLINICAL PROFILE AND SURGICAL OUTCOME FOR PULMONARY ASPERGILLOMA: A RETROSPECTIVE OBSERVATIONAL STUDY IN A TERTIARY CARE HOSPITAL}

Dr Nadeem Ul Nazeer

Department of CTVS, SKIMS Institute,

Srinagar, J and K, 190011

Background:

The indications and the outcome of surgery for pulmonary aspergilloma remain highly controversial. This retrospective observational study was conducted to study the clinical profile, indications, post-operative complications and long term outcome of patients having pulmonary aspergilloma.

Methods:

From January 2000 to October 2018, 92 patients underwent surgery for pulmonary aspergilloa at our tertiary care institute.

Results:

The group consisted of 57 males and 35 females with a mean age of 38.3 +- 11.6years. The most common indication for surgery was hemoptysis $(96.15 \%)$. The underlying lung diseases were tuberculosis $(73 \%)$, bronchiectasis $(6.76 \%)$, and lung abscess $(6.76 \%)$. In one patient $(2 \%)$, concomitant ruptured lung hydatidcyst and an aspergilloma was present. The procedures performed were lobectomy $(n=62)$, bilobectomy $(n=5)$. pneumonectomy $(n=3)$, segmental resection $(n=22)$. The post-operative mortality was $2 \%$ (two patients). Overall complications occurred in 22 patients. The complications included prolonged air leak, bleeding, empyema, repeated pneumothorax, and wound dehiscence. There was no recurrence of disease orhemoptysis.

Conclusion: Pulmonary aspergilloma is common in developing countries like India in which there is high prevalence of pulmonary tuberculosis. Surgical resection of pulmonary aspergilloma iseffective in preventing recurrence of symptoms including hemoptysis. We recommend early surgical resection of symptomatic aspergilloma with reasonable complications. Pre-operative preparation of the patients, meticulous surgical technique and post-operative chest physiotherapy reduces the rate of complications. Complications maystill occur and are largely related to the underlying lung pathology; however, the long term outcome is good.

\section{CORONARY CAMERAL \\ FISTULA : A RARE ENTITY \\ DR Divyesh Rathod, \\ DR Jignesh Kothari, DR Jigar Shah \\ U N Mehta Institute of Cardiology \\ and Research Center}

Objectives: the purpose of this study was to describe our experience with the presentation and management of surgical repair of coronary cameral fistula $(\mathrm{CCF})$ and the outcomes.

Patients and Methods: We performed a retrospective study from January 2015 to January 2018 in 8 patients operated for coronary cameral fistula . There were 5 men and 3 women; the age range was 18-55years (mean age, $36.2 \hat{\mathrm{A}} \pm$ 11.7years). Among the 8patients, 4 patient had CCF originating from Right coronary artery, 2 patients had from Left anterior descending artery and 2 patients had its origin from Left circumflex artery. All patients were evaluated by trans-thoracic echocardiography and conventional coronary angiography apart from routine biochemistry and hematological investigations.

Results: All operations were performed using cardiopulmonary bypass. Four patients with a CCF opening into RA and RV which was closed by right atriotomy, 2patients had CCF at termination of coronary artery hence ligation done and 2 patients had CCF with dilated coronary artery amongst them dilated portione xposed, closed fistulous tract followed by arteriotomy closure. There were no postoperative complications. No recurrences have been observed so far in follow up visits.

Conclusions: Coronary cameral fistulla is an in frequently encountered entity, but with clinical suspicion, early diagnosis and timely intervention it can be successfully managed with excellent outcomes.

\section{STERILE AORTIC ANNULAR ABSCESS CAVITY-“ AN INCIDENTAL INTRA-OP FINDING}

Dr. Yogesh T, Prof. Durga prasad reddy, Dr. Mayuri, Dr. Vikas S R, Dr. Subhash, Dr. Sathya, Dr. Veena

Vydehi Institute of Medical Sciences \& Research Centre 


\section{INTRODUCTION:}

Aortic abscess in patients with aortic endocarditis is not uncommon. The condition may be misdiagnosed as sepsis, pneumonia, or non-valvular heart failure. In addition, examination findings typically seen in chronic regurgitation like chamber enlargement are absent or subtle, and the murmurs are frequently soft. The formation of aortic annular abscess is the most devastating complication of destructive aortic valve endocarditis (AVE) and requires extensive surgical debridement. The chances of detection of aortic abscess on a routinepre-op echocardiography are quite subtle and is usually seen incidentally intra-operatively. Management protocol when identified depends on the number of valve cusps involved. In this study we would like to share our experience with the sterile aortic abscess that was found incidentally.

\section{MATERIALS AND METHODS}

A 57 year old patient who came with atypical symptoms went through routine pre-operative work up and was planned for aortic valve repair. Intra operatively, sterile abscess cavity was noted under the non coronary cuspof the aortic valve. Abscess cavity was debrided and aortic valve replacement was done. Procedure was uneventful.

\section{CONCLUSION}

This report reiterates that high index of suspicion is needed to detect aortic annular abscess on 2D-echocardiography, and treating with debridement with aortic valve replacement has a good prognosis.

\section{CORONARY ARTERY BYPASS GRAFTING IN A PATIENT WITH POLYCYTHAEMIA VERA}

Dr Abhishek manmohan sahu,

Ketana V. Rajasekar Rao 1,

Sruthi Kurakula.1Muntimadugu T. Babu.

1, Usharani Guntuboina. 1 Srinivasan R. vangipuram 2

1. Department of cardiothoracic surgery, CARE

Hospital,Nampally, Hyderabad 2.. Department of

Internal medicine CARE Hospital, Nampally, Hyderabad

\section{Introduction}

Polycythemia vera [PV] is a rare Myeloproliferative disease with increased production of of red blood cells, platelets and white blood cells. Platelets counts are increased but their function is impaired. They may be symptomatic or asymptomatic. Occasionally patients needing coronary bypass surgery may be having known PV or diagnosed at the time of admission because high Haemoglobin. Case Report52 year old male patient was admitted with acute anterior wall myocardial infarction. Underwent Thrombolytic therapy and coronary angiogram showed triple vessel disease. He was considered for CABG. Haemogram done at that time showed Haemoglobin $17.5 \mathrm{gm} \%$,wbc count 18,400 , and platelet count $7,23,000$. As patient was not a smoker possibility of polycythaemia vera was considered . Based on positivity for JAK2 mutation polycythaemia vera was confirmed. He was started on low molecular weight heparin prior to surgery. Underwent off pump cabg x3. Saphenous vein had localised thrombosisat both ankles. No blood transfusions were given in the periop period hemoglobinat discharge was $9.5 \mathrm{gm} \% \mathrm{He}$ was started on clopidogrel soon after surgery and acitrom was started on po day 3.He was started on antineoplastic agent hydroxyurea 2 weeks after surgery. His hemogramin the follow up 2 months after showed all counts with in normal limits and he had no cardiovascularevents in the followup period of 3 months Discussion Polycythaemiavera could be asymptomatic and hemoglobin above 16 may warrant further assessment. Assessment of erythropoietin levels and JAK2 mutation levels could help in differentiating primary and secondary polycythaemia. When polycythemiavera is diagnosed using anti thrombotic agents and anti neoplastic agents in the in the perioperative period will be needed Key words Polycythaemia vera Coronary artery bypass grafting, Jak2
AORTIC VALVE REPLACEMENT IN POST RENAL TRANSPLANT PATIENT: CASE REPORT

Neraj kumar Jha, Lalit kapoor, Dhiraj barman, Devraj Kumar, Sathish Kumar, Sufina Shales. Department of cardiac surgery, Rabindra Nath Tagore International Institute Of Cardiac Sciences, Kolkata 700099

\section{Aims/ Objectives}

Post renal-transplant patients undergoing aortic valve replacements pose significant challenges both in the short and long term. The aim of this report is to identify factors that help in conducting aortic valve replacement in this patient population with minimum morbidity and satisfactory long term outcomes.

\section{Material and Method}

Our report describes implantation of a mechanical aortic valve in a patient who had undergone renal transplantation 15 years ago. Strategy regarding immuno-suppression and graft rejection, systemic infection, maintaining adequate perfusion pressures, avoidance of nephortoxic drugs and close monitoring of renal function in close liaison with nephrologists is essential for successful outcome. Decision making regarding choice of valve prosthesis is of paramountsignificance.

\section{Results}

Mechanical valve prosthesis was used to reduce immunogenicity and improve durability. Cardiopulmonary bypass time is directly linked to poorer outcome and hence continuous suturing technique to reduce the cross clamp time (38 mins.) and bypass time (51mins.) was undertaken. Immuno-modulators were allowed until morning of surgery. Nephrotoxic drugs and vaso-constrictors in the post operative period were avoided. Dopaminewas preferred for inotropic support. Immuno-suppressants were reintroduced on the first post-operative day. Strict aseptic precautions and attention to haemostasis was undertaken to prevent blood transfusion and resultant immune modulation. Post operative recovery in our patient was uneventful. At 3-months follow-up patient remains well with a significant improvement in NYHA class.

\section{Conclusions}

Valve replacement in the setting of renal transplant is safe. However, strategy for a successful outcome is dependent on many factors and requires multi-disciplinary input.

ENDOCARDIAL TUBERCULOSIS: DIAGNOSTIC CHALLENGE

Dr Madhur Kumar, Dr Aabha Divya

(ASSISTANT PROFESSOR),

Dr Anubhav Gupta (Professor \& Head)

Safdarjung Hospital, New Delhi

\section{Introduction-}

Tuberculosis (TB) involving the endocardium is extremely rare. Case- We report a case of 55-year-old female was admitted with a two month history ofshortness of breath and fatigability. Her routine investigations and chest $\mathrm{x}$-ray were normal. Transthoracicecho cardiography was suggestive of right atrial mass. Cardiac MRI with contrast was performed which revealed a solitary, soft tissue lesion, $5.3 \times 1.5 \mathrm{cms}$ abutting right atriallateral wall. There was no evidence of calcification or pericardial and pleuraleffusion, suggestive of either myxoma or sarcoma. Endomyocardial biopsy was inconclusive. Open biopsy via median sternotomy of right atrial mass was done. Histopathological examination of the specimen revealed granulomatous inflammation including epitheloid cell granuloma, langhans giant cell with caseating necrosis, but no signs of malignancy. TB culture showed growth of fully sensitive strain and TB treatment was started with isoniazide, rifampicinand pyrazinamide in recommended dosage. At follow-up, 6 months 
after completion of treatment, the patient was fully recovered with normal inflammatory parameters and without any signs of relapse clinically.

Conclusion-Confirming the diagnosis of myocardial tuberculoma requires culture from the infected tissue. Myocardial tuberculoma might be successfully treated with anti-tuberculosis drugs only. Indications for surgery include uncertain diagnosis, poor response to medical treatment or cardiac complications.

\section{THE ROLE OF THYMECTOMY IN THE TREATMENT OF MYASTHENIA GRAVIS - A SINGLE CENTER EXPERIENCE.}

Dr. Dinesh kumar. R, Dr. Anagha Tulsi A., Aditya Doddamane, Dr. Naveen. P, Dr. Hiremath. C.S, Dr. Shekhar Rao.

Sri Sathya Sai Institute of higher medical sciences

\section{Background}

Myastheniagravis (MG) is an autoimmune disorder characterized by skeletal muscle weakness, which affects the neuromuscular junction. The treatment in MG consists of anti-cholinesterase, immunosuppressants or by surgical removal of thymus since it is considered to play an important immunopathological role in the development of autoimmune MG. We present our analysis in patients who had undergone thymectomy at our institution.

\section{Objective}

To study the effectiveness of thymectomyin myasthenia gravis patients in terms of symptomatic improvement and quality of life.

Methods: From April 2001 to April 2019, 81 patients with myasthenia gravis have undergone thymectomy in the Department of CardioThoracic\&amp; Vascular Surgery, Sri Sathya Sai Institute of Higher Medical Sciences, itefield, Bengaluru. Those patients who were treated medically were not included in this group. Mean age at which they underwent surgery was 26.6 years $+/-19.02$ years. Thirty-five patients $(43.2 \%)$ were females and 46 patients $(56.79 \%)$ were males.

\section{Results}

Clinical improvement from symptoms was observed in all (100\%) patients after a period of 13 months post-surgery. In 43(59.72\%) patients, the gradual improvement of symptoms rendered the reduction of immunosuppressants dosage by 15 months after surgery. The remaining 29 patients $(40.27 \%)$ who had recurrent acute exacerbations despite high dosage of medications became asymptomatic with the same regimen of preoperative drugs. Among 5 patients had received plasma exchange treatment preoperatively, on an average of 2 times per person after surgery, the requirement for plasma exchange for 4patients was zero. One patient developed myasthenia crisis needing reintubation and prolonged ventilation. To tide over the crisis, patient was treated with IV immunoglobulin therapy to facilitate extubation. Due to persistence of symptoms she underwent plasmapheresis. After 3 months, she had significant improvement in symptoms with no relapses. Conclusion

Although the exact mechanism behind thymectomy is unknown, it is found to have improved the response to medications in patients with uncontrolled symptoms. Patients are found to have prolonged periods of remission. There is reduced need for invasive procedures of plasmapheresis and reduces side effects due to high dose of immunosuppresants. Thymectomy is beneficial for patients in controlling the symptoms, reduction of immunosuppressants dosage, achieving the remissions and relapses. Overall thymectomy improves the quality of life in victims of myastheniagravis.

RHEUMATIC TRICUSPID VALVE: IT IS THE PATIENCE THAT COUNTS

Alok Mathur, M.Ch., Akshay Sharma, M.Ch; Harish Khanna, M.D., Sushil Chhabra, M.D. CK Birla Hospitals / RBH, Jaipur: 302018, India

\section{Aim}

To device a standard algorithm for surgical management of rheumatic tricuspid disease.

Methods

350 patients underwent valvular heart surgery (mitral valve replacement (MVR)/Double valve replacement (DVR)) from Jan 2014 â€“ July 2018.Out of these 72 underwent concomitant tricuspid valve (TV) repair. 2 patients had isolated TV surgery and had an earlier MVR. Indications for tricuspid valve inspection and repair included rheumatic involvement, moderate or more regurgitation with annular dilatation and mild or more regurgitation without annular dilatation. Three surgical techniques were employed as per the pathology, (a) Commisurotomy, (b) Pericardial augmentation of Anterior Tricuspid Leaflet (ATL), (c) De-Vega Tricuspid Suture Annulopalsty or ring annuoplasty.35 underwent isolated commisurotomy, 12 had commisurotomy andannuloplasty, 27 patients underwent a combination of ATL augmentation with commisurotomy and annuloplasty.

\section{Results}

There was no surgical mortality and none of the patient required a TV replacement. 73 out 74 patients are in follow up. All patients are in functional class NYHA I or II.

\section{Conclusion}

A surgical algorithm is proposed and has been subsequently published. Following this, a competent tricuspid valve could be achieved in all patients and consequently diuretics were withdrawn.

The salient feature of the study is limitation of trans-thoracic and transesophageal echocardiography in planning the exact surgical procedure. Of importance is the group with mild or more regurgitation in absence of annular dilatation. Most of these patients had advanced tricuspid disease.

\section{SUCCESSFUL MANAGEMENT OF PREGNANCY IN WOMEN WITH TRANSPOSITION OF GREAT ARTERIES (TGA) AFTER ATRIAL SWITCH OPERATION}

Dr Gaurav Patni, Dr Lalit Kapoor,

Dr Pradeep Narayan, Dr Sathish Kumar,

Dr Sufina Shales PK

NH Rabindranath Tagore International Institute

of Cardiac Sciences, Kolkata

\section{Aim/Objective(s):}

With a growing number of women approaching childbearing age, it is important to be aware of the increased cardiovascular risks pregnancy poses in women after complex congenital surgery like the Senning operation. In this study we have formulated a strategy for prevention of any cardiovascular complications in this cohort of patients.

\section{Material and method:}

A 20 year old pregnant lady with a history of TGA with intact septum and had undergone Sennings operation at the age of 7 years, presented to our hospital. Upon becoming pregnant a close liaison with the obstetrician, paediatric cardiologist and cardiac surgeon was organized. Preoperative echo was regularly performed to assess baffle obstruction along with late systemic right ventricular failure which is one of the most serious late complications of the Senning operation. Fetal monitoring was done, antibiotics prophylaxis was given for infective endocarditis and diuretics were used to affect the stress of labour and potential of adverse events like heart failure, arrhythmia, thrombo-embolic events and, worsening systemic ventricular function it was decided to carry out elective caesarian section. Close monitoring of arterial blood pressure and continuous cardiac output was performed.

\section{Result(s):}

The entire process of child birth and recovery was uneventful. No signs of heart failure were seen and the child was of normal weight. After two month follow up both mother and child are doing well. 


\section{Conclusion:}

Pregnancy is well tolerated after Senning operation. However, to minimize the potential of adverse aternal cardiac events a multidisciplinary approach with a well formulated strategy needs to be in place.

\section{PRAGMATIC MANAGEMENT OF ANOMALOUS ORIGIN OF RIGHT CORONARY ARTERY WITH SEVERE AORTIC STENOSIS.}

Ravi Shankar, Korah Kuruvilla, Roy Thankachen, Vinayak Shukla Christian Medical College and Hospital, Vellore, Tamil Nadu, India

Abstract: Anomalous origin of the coronary artery is infrequent, the most common of them being of the left coronary artery. Right coronary artery arising from the pulmonary artery are even more rare. They are usually asymptomatic and detected incidentally or with a coexisting condition. We report a case of right coronary artery arising from the pulmonary artery associated with severe aortic stenosis. Key-words: anomalous coronary arteries, anomalous origin of right coronary artery, aortic stenosis Key Messages: Uncommon case, simple management. Disclosures : Nil Introduction: Anomalous origin of coronary artery from the pulmonary artery is a rare anomaly that most frequently involves the left coronary artery and very rarely the right coronary artery. The incidence of abnormal aortic origin of the coronary arteries have been reported to be approximately $0.64 \%$ of births. 1 Anomalous right coronary artery from the pulmonary artery (ARCAPA) is a rare congenital coronary anomaly defect first described in 1852 with a reported incidence of only $0.002 \% .3$ Many patients with isolated ARCAPA remain asymptomatic and it is diagnosed incidentally with other types of cardiac anomalies. Twelve cases were diagnosed in infants â\%ol year of age, 44 were diagnosed in children â $\%$ o1 18 years of age, 17 were adults \&gt; 60 years of age, and eight subjects were of unknown age. [5] Massive collateralization between the normal and anomalous vessel is needed to maintain adequate myocardial perfusion in the presence of coronary steal. Coronary steal occurs when the anomalous vessel acts as a vein, which collects blood from the normal coronary artery through collaterals and delivers it to the pulmonary artery. Patients with associated cardiac anomalies are diagnosed early in life compared to patients with isolated ARCAPA. Those without associated cardiac defects may present with heart murmur, congestive symptoms, sudden cardiac death or may remain asymptomatic and detected incidentally during evaluation of other problem (e.g. coronary angiography in the older age group). [6] Associated cardiac defects were reported in $22 \%$ of the patients reported with ARCAPA. The cardiac defects found in these patients were aortopulmonary window, tetralogy of Fallot, VSD, PDA, and aortic stenosis. [7] ECG in ARCAPA may be normal or it may show left ventricular hypertrophy or deep $\mathrm{Q}$ waves in inferior leads. [8] Case History: This 54-year-old lady, presented with history of breathlessness on exertion, NYHA class II, which had worsened over the last 6 months. There were no other significant illnesses in the past. Physical examination of the patient revealed the apical impulse in the 5th LICS $2 \mathrm{~cm}$ lateral to the midclavicular line with a grade $3 / 6$ ejection systolic murmur is heard in the aortic area conducted to the carotids. Chest x-ray (Figure 1) showed a cardiothoracic ratio of 0.58 with lung plethora. Figure 1 : Cardiothoracic ratio 0.58 with mild lung plethora. The Echocardiography showed AS Severe, TR Moderate, Pulmonary Hypertension with a TR max PG of $47.0 \mathrm{mmHg}$ and a normal LV Systolic Function. The cardiac catheterisation study showed a PA pressures of $42 / 20 / 29 \mathrm{mmHg}$. The coronary angiogram showed both the $\mathrm{LAD}$ and $\mathrm{LCx}$ was dilated and tortuous. Through multiple collaterals were draining to RCA into PA. The RCA was not originating from the Aorta. Probably draining into PA. The Aortic root angiogram showed a non-dilated aorta. No Aortic regurgitation. RCA seemed to be draining into MPA. (Figure 2) The impression made was of a probable ARCAPA (Anomalous origin of Right Coronary Artery from Pulmonary Artery with mild Left to right shunt, the Qp: Qs being 1.09.Figure 2.The CT
Angiogram (Figure 3 ) showed the right coronary artery arising from the main pulmonary artery. The proximal RCA has an inter-arterial course between the main pulmonary artery and the proximal ascending aorta (for a length of about $5 \mathrm{~mm}$ ). Rest of the RCA is ectatic and is seen to course caudally in the right $\mathrm{AV}$ groove. Figure 3.The patient was consented and a decision to do an aortic valve replacement with suture occlusion of the ARCAPA origin with a SVG graft to RCA was taken. Standard aortobicaval cardiopulmonary bypass was instituted. The LV was vented through the RSPV. The heart was arrested with antegrade aortic root cardioplegia. The Proximal RCA was identified, a small arteriotomy made and a segment of vein was anastamosed here. An. Oblique aortotomy made. The aorta valve cusps were excised and the annulus was decalcified. The aortic annulus was very narrow, measuring about $17 \mathrm{~mm}$ diameter. The aortic root and annulus were augmented using the patientâ $€^{\mathrm{TM}_{\mathrm{S}}}$ pericardium. After augmentation the aortic valve was replaced with a bileaflet size 19 aortic valve. The origin of the RCA was closed from inside the Pulmonary artery. Patient had an uneventful postoperative period. An Echocardiogram on follow-up after 6 months showed a Normally Functioning Prosthetic Aortic Valve, DVI - 0.44, TR Moderate; PAH with a TR Peak Gradient of $39.16 \mathrm{mmHg}$. Discussion: In 1945, Soloff described four possible types of anomalies of the coronary artery originating from the pulmonary artery. These can be an anomalous origin of the left coronary artery from the pulmonary artery (ALCAPA), right coronary artery from the pulmonary artery (ARCAPA), both coronary arteries from the pulmonary artery and an accessory coronary artery from the pulmonary artery. Among these, ALCAPA is the most common anomaly, with an incidence of $0.008 \%$ in the general population compared to a rate of $0.002 \%$ for ARCAPA. Only $25-30 \%$ cases of ARCAPA are associated with structural heart defects. [4] The pointers for the diagnosis were the mild plethora of the lung fields, moderate TR with mild PAH in the presence of severe aortic stenosis with a normal LV function. The routine coronary angiogram done before an aortic valve replacement made the diagnosis which was then anatomically defined using a Cardiac CT. The operative management done was to keep things simple. The ideal management of transferring the RCA origin to the aorta was complicated by the need of an aortic valve replacement. This was also compounded by the thinned out aorta in a fragile 54-year lady. An alternative of unroofing of the proximal RCA into the aorta was again decided against as an aortic valve replacement with the narrow root would make surgery difficult and dangerous.

\section{OFF PUMP TOTAL ARTERIAL REVASCULARISATION IN SEVERE LEFT VENTRICULAR DYSFUNCTION}

Shubhi Bhatnagar, Prateek Bhatnagar

Beating Heart Clinic, Hyderabad

Aim/Objective: Severe left ventricular dysfunction has been an increasing trend in patients with coronary artery disease. Off pump coronary artery bypass grafting has been shown to reduce the risk in presence of severe left ventricular dysfunction. Total arterial revascularisation has shown good long term benefits. We aimed to analyze our early results after off pump total arterial revascularisation in severe left ventricular dysfunction patients. Material and method: 3368 patients with left ventricular ejection fraction less than $30 \%$ were included in the study between January 2003 to December 2012.. Lowest left ventricular ejection fraction was $18 \%$. Total arterial revascularisation was provided with a left internal mammary artery/right internal mammary artery " $\mathrm{Y}$ " graft, a left internal mammary artery/ radial artery " $\mathrm{Y}$ " graft or a conventional arterial grafting technique. Different variables ( post surgery mortality, complications, conversion rates to cardiopulmonary bypass, rates of re exploration) were evaluated. Results: The commonest age distribution was 61 to 70 years. $71.8 \%$ of the patients were males. An average of 3.36 grafts were performed per patient. 226 patients underwent left internal mammary artery/ radial artery " Y" grafting. 97 patients underwent left internal mammary 
artery/ right internal mammary artery " $\mathrm{Y}$ " grafting. The rest underwent conventional arterial grafting. Post operative complications ,conversion rates to cardiopulmonary bypass and mortality rates were evaluated. Conclusion: Off pump total arterial revascularisation can be done in patients with severe left ventricular dysfunction, with low morbidity and low hospital mortality.

\section{SINGLE CENTRE EARLY EXPERIENCE OF ROBOTIC ASSIST MINIMALLY INVASIVE CORONARY ARTERY BYPASS GRAFT SURGERY \\ Dr. Paulami Sen, Dr. M. M. Yusuf \\ Apollo Hospital, Main}

Objective: Minimally invasive cardiac surgery is being done more frequently nowadays owing to its significant advantages in reducing surgical trauma. Robotic assisted surgery offers even lesser pain and only a one- or two-day hospital stay post operatively. Return to normal activities is much faster for the patients. We present our early experience using this technique in patients requiring coronary revascularisation. Methods: 18 patients were treated by Robotic Assist Minimally Invasive Coronary artery bypass graft surgery over a period of 6 months. Da Vinci Robotic System is used to harvest the left internal mammary artery followed by extension of one of the middle ports for direct hand-sewn anastomosis. Robotic assist minimally invasive hybrid procedures are also performed for double vessel diseases where it is a 2 staged procedure, the first stage being surgical anastomosis using a robot. The patients are then loaded with antiplatelets on POD 2 after which drug eluting stents are used to perform PCI to the non $\mathrm{LAD}$ vessels. As there is no rib retraction, the pain post-surgery is even lesser than that of Minimally invasive surgery with a much shorter post-surgery hospital stay. Patients can return to most of their daily presurgery activities within a week. Suitable patients with single or double vessel coronary artery disease are assessed by the cardiac team comprising of a cardiac surgeon, cardiologist and cardiac anaesthetist before confirming surgical procedure. Result:4 out of the 18 patients were females. Left Internal Mammary artery was harvested in all patients. For 1 patient, the mammary flow was inadequate hence saphenous vein graft was used to bypass LAD through sternotomy and the procedure needed to be converted to open CABG.6 out of the 18 patients underwent Hybrid Robotic Assist Minimally Invasive CABG. 1 patient had a euro score of 3 and he was discharged on second post operative day. Requirement of post operative analgesia is lesser than that of Minimally invasive surgeries. There was no mortality or re exploration or post operative myocardial infarction. The maximum follow-up is 6 months, The median follow up is 3 months. Conclusion: Robotic assisted minimally invasive cardiac surgery offers significantly less pain, shorter hospital stay and early return to daily activities. It is a safe procedure and more convenient for patients belonging to middle age group and having coronary artery diseases because of early recovery and early return to work.

\section{SURGICAL INTERVENTION FOR ANOMALOUS ORIGIN OF LEFT CORONARY ARTERY FROM THE PULMONARY ARTERY IN PEDIATRIC AGE GROUP-MID TERM OUTCOME}

Shivang Saxena, Sowmya Ramanan MS,Mch, Baiju S Dharan MS,Mch (Head of department), Sabarinath Menon MS,Mch, Sudip Dutta Baruah MS,Mch

\section{SCTIMST ,TRIVANDRUM}

\section{AIMS/OBJECTIVE'S-}

This study was undertaken to determine the mid term outcome in patients with anomalous origin of left coronary artery from the pulmonary artery (ALCAPA) undergoing repair with or without concomitant mitral valve repair.
MATERIALAND METHOD-

A retrospective review was done for patients less then 17 years age who underwent ALCAPA repair at SCTIMST, Trivandrum , between January 2003 to December 2012.Preop echocardiographic findings-LV dimension, coronary anatomy, severity of mitral regurgitation (MR) were studied and compared to 5 year post operative echo findings.

RESULTS-

Total50 patients were studied out of which 5 have been lost to follow up and rest 3 had no previous records in hospital database. The median age at time of surgery was 7 months (mean -42 months,range -1 month to 16 years). Repair of ALCAPA was done by re-implantation of anomalous coronary in $100 \%$ cases. 6 patients (14\%) had concomitant MV repairat time of initial operation. The mean age of patients who underwent concomitant MV repair was 21.5 months (range 3 months to 36 months). Survival was $100 \%$ at 5 years of follow up. 8patients(19\%) had severe mitral regurgitation pre operatively out of which 3 patients $(37.5 \%, 3 / 8)$ underwent concomitant mitral valve repair. Other 4 had mild MR at 5 year follow up and one with MR.100\% patients had good LV function (EF\&gt;60\%) at time of 5 year follow up. CONCLUSION-

ALCAPA can be operated with good outcomes and only persistent or moderate mitral regurgitation requires close medical follow up.

\section{DESIGN, DEVELOPMENT, FEM ANALYSIS OF HYBRID 12/14 BSRM WITH ADVANCED CONTROLLERS FOR SAISPANDAN-THE GITAM HEART- BEARING-LESS MAGNETIC INDIAN TOTAL ARTIFICIAL HEART FOR DESTINATION THERAPY (DT)}

Pradeep Kumar Radhakrishnan, Sujatha Mohanty,Pulivarthi Nageshwar Rao,Siva Krishna Rao G V, Nagesh Kuma,Bisoi A K, Siva Prasad,Satya Narayana Murthy, Satya Narayana M R S,Ravi Shankar,Srinivas, Das P K,Valluvan Jeevanandam, Venugopal Pangapply

Gimsr, Gitam University, Visakhapatnam, AP, India

Rotor vibration control during start-up, acceleration and deceleration phases are one of the key problems besides stable levitation, in high-speed applications of bearing less switched reluctance motor (BSRM) .Global sliding mode controller (GSMC) is proposed to control the speed and position of BSRM. Sensor less operation is achieved with sliding mode observer. Rotor displacement tracking error functions were used in the sliding mode switching functions. New sliding mode displacement control and speed tracking equations obtained using extra exponential fast decaying nonlinear function and conventional linear sliding mode switching. Simulation is carried on hybrid12/14 BSRM with the proposed controller and observer. 12 stator poles and 14rotor poles were selected. Pole arc was 25.71.FEM analysis showed Torque polearc was 12.85.Air gap length was 0.25 $0.5 \mathrm{~mm}$. Stator diameters $-112 / 60.2$, statoryoke thickness $7.7 \mathrm{~mm}$, shaft diameter of $18 \mathrm{~mm}$, coil width of $4 \mathrm{~mm}$, rotor pole arc of 12.85 , axial stack length of $40 \mathrm{~mm}$, rotor yoke thickness of $9.7 \mathrm{~mm}$-were the major design parameters. FEM of motor parameters under different loads for speed, torque load, switching angle, voltage, phase resistance and moment of inertia was performed. Sensorless method was used to reduce the manufacturing complexity. New robust controllers DSMC and GSMC were used to control rotor displacements and speed and SMTO for avoiding mechanical sensors and reduce the complex controlling processes

\section{A RARE CASE OF INCIDENTALLY DETECTED PRIMARY CARDIAC ANGIOSARCOMA OF RIGHT ATRIAL APPENDAGE}

Dr Srawanthi Ponnuru, Dr Renjith S, Dr Vivek V Pillai

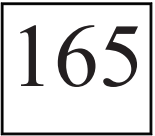

Sree Chitra Tirunal institute for medical science and technology, TRIVANDRUM 
Background-

Primary tumors of the heart are extremely rare, occurring at afrequency of $0.02 \%$ in autopsy series and the majority of them are benign. Metastatic heart tumors are 20-40 times more frequent than primary cardiac tumors . Statistically, heart cancer is listed with all cancers of the soft tissues. Malignant tumors of the heart are most often sarcoma (76-78\%) andangiosarcoma, the most aggressive histotype, accounts for about $31 \%$ of all primary cardiac malignant tumors.

Case Report-

A 67 year old male patient presented with complaints of chest pain and was diagnosed to have Myocardial infarction and was taken up for elective coronary artery bypass grafting. Intra operatively found to have a mass of size $3 \times 3 \mathrm{~cm}$ in the right atrial appendageclose to SVC-RA junction . Right Atrial appendage mass was excised along with a cuff of normal tissue and sent forHisto pathological examination, which was found to be a Vasoformative neoplasm-a well differentiated angiosarcoma . Immunohistochemistry was CD34:Positive, CD31:Positive MIB-1: 3$5 \%$. Patient was further investigated for metastatic masses but $\mathrm{CT}$ of chest, brain and abdomen. It was found to be primary angiosarcoma of heart, with no metastatic or any other primary neoplastic lesions in body. Conclusion-

Primary cardiac angiosarcomas are rare neoplasms which should be considered in the differential diagnosis of cardiac masses .BackgroundPrimary tumors of the heart are extremely rare, occurring at a frequency of $0.02 \%$ in autopsy series and the majority of them are benign. Metastatic heart tumors are 20-40 times more frequent than primary cardiactumors . Statistically, heart cancer is listed with all cancers of the soft tissues. Malignant tumors of the heart are most often sarcoma (76$78 \%$ ) and angiosarcoma, the most aggressive histotype, accounts for about $31 \%$ of all primary cardiac malignant tumors.

Case Report-

A 67 year old male patient presented with complaints of chest pain and was diagnosed to have Myocardial infarction and was taken up for elective coronary artery bypass grafting. Intra operatively found to have a mass of size $3 \times 3 \mathrm{~cm}$ in the right atrial appendageclose to SVC-RA junction . Right Atrial appendage mass was excised along with a cuff of normal tissue and sent for Histo pathological examination, which was found to be a Vasoformative neoplasm-a well differentiated angiosarcoma . Immunohistochemistry was CD34:Positive, CD31:Positive MIB-1: 3$5 \%$. Patient was further investigated for metastatic masses but $\mathrm{CT}$ of chest, brain and abdomen. It was found to be primary angiosarcoma of heart, with no metastatic or any other primary neoplastic lesions in body Conclusion-

Primary cardiac angiosarcomas are rare neoplasms which should be considered in the differential diagnosis of cardiac masses

\section{COMPLICATED RIGHT VENTRICULAR MYXOMA AND ITS MANAGEMENT -A CASE REPORT}

Prashanth Vytla, Arun Basil Mathew, Kirun Gopal,

Praveen Kerala Varma

Amrita Institute of Medical Sciences, Kochi

Aim/Objective(s) -To put forward the diagnostic and therapeutic challenges of right ventricular mass causing right ventricular outflow tract (RVOT) obstruction which is a rare entity Material and method/Case presentation -A 22 year old male with a recent history of palpitations was diagnosed to have a large right ventricular mass attached to the base of anterior leaflet, occupying most of the right ventricular cavity causing RVOT obstruction. Complete surgical excision of the mass and tricuspid valve replacement were performed. Laparotomy for abdominal decompression had to be performed. The diagnosis of myxoma was histologically confirmed. Conclusion -Cardiac myxomas located in the right ventricle with RVOT obstruction are rare and can present with unusual challenges
FLOATING TUMOR IN HEART

Dr P Vivekanand

Madras Medical College

Floating Tumor in Heart:38 yrs old female was suffering from abdominal pain, abdominal distension, breathlessness and palpitation for 9 months. On evaluation pt diagnosed to have uterine tumor invading IVC, RA\&amp;RV.Uterine tumor invaded IVC and entered into RA \&amp; RV and formed floating tumor mass within IVC, RA, \&amp; RV. Pt developed abdominal pain, abdominal distension followed by breathlessness \&amp; palpitation due to floating tumor obstructing IVC, RA \&amp; RV flow. Cardio Thoracic, Vascular, Surgical oncology and Gynaecology team was involved to remove tumor with hysterectomy, IVC exploration and tumor evacuation by vascular team and Floating tumor evacuation from RA\&amp;RV by Cardiothoracic team. HPE report showed leiomyomatosisPt is doing well.

\section{A CASE REPORT OF INTRA CARDIAC REPAIR (ICR) OF 29 YEARS BOY WITH CONGENITAL DCRV,SEVERE RVOT OBSTRUCTION, DILATED RIGHT HEART AND GOOD BIVENTRICULAR FUNCTION.}

Zubair Rashid

Krishna Institute of Medical Sciences Hospital 29 years ol boy presented with DOE for 6 months associated sweating. It releived with rest. He has no history of any chest pain,palpitations, syncope,fever,chills or rigor. No significant past,personal,childhood history.he was evaluated and found to haveDCRV with severe RVOT obstruction, dilated RA, RV, Hypertrophied RV, Good BV function (EF : 65\%) Mild TR, Trivial PR, No clots, No pericardial Effusion.ICR done. Post op period was uneventful. Patient was hemodynamically stable at the time of discharge.

\section{ECMO SUPPORT IN POST PERFUSION LUNG SYNDROME AFTER CORONARY ARTERY BYPASS} GRAFTING \& LEFT VENTRICLE CLOT REMOVAL

Anuj Sangal, Ratnamalika Kumar, Shobhit Saurav, Rajneesh Malhotra Max Superspecialty Hospital

Introduction-Post perfusion lung syndrome is a rare but sometimes lethal complication secondary to cardiopulmonary bypass. It often leads to multiorgan failure and mixed acid-base disturbances, thus making a refractory condition.

Methods-We report a 37-year-old male, undergoing a successful CABG+ LV clot removal under cardiopulmonary bypass, unfortunately developed post perfusion lung syndrome with associated multiorgan failure and triple acid-base disturbances. His condition deteriorated rapidly on postoperative day one and became moribund. This article reports a rare association following on pump $\mathrm{CABG}+\mathrm{LV}$ clot removal, complicated with post perfusion lung syndrome and high-anion-gap and hyperchloride. Results-Institution of VV-ECMO with low tidal volume $(6 \mathrm{~mL} / \mathrm{kg})$ in order to support the lungs and minimize the compromise to cardiac function. Patient was successfully weaned off and discharged and now on regular follow-up.

\section{MYASTHENIA GRAVIS AND} SURGICAL COMPLICATIONS

Dr. S. Raghupathi, Dr. MS Senthil KUmar

Heart Institute, Aynavaram, Chennai.

The Surgical Management of Myasthenia Gravis

What is MyastheniaGravis? Myasthenia gravis is a disorderof neuromuscular transmission characterized by weakness and fatigue ofvoluntary 
muscles. It is now reasonably established by due to an autoimmuneattack directed against postsynaptic nicotinic acetylcholine receptors of voluntary muscles.

A relationship between myastheniagravis and the thymus gland has been appreciated since at least 1901 .

Clinical Features-Incidence- 1:75,000, Morecommon in young women and elderly men, Females : males $2: 1$,Males are associated with high incidence of HL Aantigens Weakness and fatigue with activity (exercise) are the hall-marks of myasthenia Common muscular Ocular muscles / ptosis and diplopia Other cranial nerves may also be affected leading to potentially fatal complications such as dysphasia and respiratory distress. Facial weakness with a transverse smile and involuntary grimace may develop. Tongue may become atrophic with acharacteristic triple furrows. Weakness of the flexor and extensor of the limbs. Autonomic system changes like pupilary change,bladder disturbances and increased sweating may also occurs. A transient neonatal myasthenia gravis has been reported.

A congenital myasthenia gravis mothers are not affected but the babies are affected.

Drugs for congestial myasthenias are more useful. Eaon-Lambert syndrome: Weakness and fatigability of proximal muscles in the lower extremities

Clinical Presentation of Myasthenia Gravis

Group I : Ocular Myasthenia Only ptosis and diplopia o mortality.

Group II : (A) Mild Generalised Ocular and spreading to skeletal and bulbar muscles

Respiratory system not involved Response to drug therapy. Low mortality rate (B) Moderate GeneralisedA plus dysarthria, dysphasia and difficult mastication Response to drug therapy less patientâ $€^{\mathrm{TM}_{\mathrm{S}}}$ activities restricted (C) Severe Generalised Severe onset of bulbar and respiratory muscle weakness. Respiratory muscle Involvement and thymoma is highest Severe myasthenia gravis after 2 years. Poor response to drug therapy Myasthenia Gravis differential diagnosis Eation-Lambert syndrome Hysteria Thyroid Neuromyopathies Associated Conditions Rheumatoid Arthritis, SLE, Polymyositis, Sjogren's Syndrome, Ulcerative colitis Vitamin B, deficiency Thyroid parathyroid and adrenal disease Associated more likely immunological or goitre diseases Thymic Abnormalities Thymus Gland is involved in $75 \%$ of Myasthenia Gravis. Thymus may be benign, encapsulated, cystic and calcified. Malignancy thymomas defined by tumor infiltration intopleura, pericardium confirmed by histologic pattern. Commonly not seen in children especially before the age of 30 .

CT Scan must for early diagnosis, Lymphoid hyperplasia both in cortex and medulla is found in Thymus. Associated with T-cell and B cells. After 55 years thymus gland is atrophic identified by CT scan. Atrophic gland may be still active and also associated with $3 \mathrm{~A} 1+$ OKT4 cells reversed after thymectony.

General Treatment of Myasthenia Gravis-Drug therapy 61\%, Plasmapheresis, Surgical treatment, Medical treatment : Anticholinesterase agents introduced in 1930 common drug.

Neostigmine : $15 \mathrm{mg}=4$ hours (Prostigmin ) Pyridostigmine (Mestinon) Neostigmine: $0.5 \mathrm{mg}$ IV $=15 \mathrm{mgs}$ oral $\mathrm{a} €^{\prime \prime} 60 \mathrm{mgs}$ of pyriodostigmine $=$ $15 \mathrm{mg}$ of neostigmine may delay thyectonmy procedures.

Side effects :Diarrhoea, nausea salivations and locomotion disturbance Corticosteroids: Better be started inhospitals. Common drugs Prednisone 60 to $80 \mathrm{mgs} /$ day After adequate response-alternate day therapy. Corticosteroids are more useful for preparation for Thymectomy. Plasmapheresis and sometimes it may act equivalent to Thymectomy Side effect of Corticosteroids: Cataracts, phychosis, gastrointestinal bleeding, carbohydrate intolerance, hypertension, obesity osteoporosis, growth failure in children, resistance to infection cushingoid features etc.

Plasmapheresis: A technique of selective removal of plasma components by the centrifugal method. The cells are suspended in Ringer's solution and then reintroduced to the patient. 1 to 3 liters of plasma is removed per run onalternate day basis until the maximal benefits are obtained (usually 4 to 6 runs). Hypotension is the possible problem (1 or 2 nd run). Hypocalcemia and Hypoalbuminemia. Thymectomy is avoided $48 \mathrm{hrs}$ minimize to avoid the riskof bleeding and infection.

Plasmapheresis Achieves : Only temporary benefit. Causes clinical remissionuseful in refractory condition of thymectomy.

Action : It helps to the removal the acetylcholine receptor antibody.

ImmunosuppressiveDrugs: Antilymphocyte -1.5 to $3 \mathrm{mgs} / \mathrm{kg}$, Cyclophosphamide

Side effects : GI bleeding, resistant to infection, malignancy and death More effective along with plasmapheresis Measures of antilymphocyte, antithymocyte serum and splenic radiation have all been tried for immunosuppression.

Thymectomy The thymus gland contains myoid cells. These cells contain AchR and react with AchR antibodies. These cells are proximal tolymphocyte maturation and causes antigen and antibody reaction. This produces killer Tcells and helper cells. These destroy the neuromuscular function.

Thymectomy helps To remove killer, T-cells and helper cells, AchRantigen and antibodies causing destruction of neuromuscular function are removed. Thymectomy helps these events not to happen there by prevents Myasthenia Gravis.

Conclusion Myasthenia Gravis patients can be treated only with experiences cardiothoracic surgeon with good ICU faculties.

\section{GOLDENHAR SYNDROME WITH \\ MULTIPLE SKELETAL DEFECTS AND ITS RARE ASSOCIATION OF CONGENITAL HEART}

\section{DEFECT- ATRIAL SEPTAL DEFECT(ASD).}

Dr. Sana Firdouse, Dr. Alla Gopala Krishna Gokhale, Dr. Koneru Sudhakar, Dr.Pavaneel Bhandari.

Apollo Hospitals, Jubilee Hills.

Aim: To present our experience with a case Goldenhar syndrome with multiple skeletal defects and rare association of congenital heart defectAtrial septal defect (ASD) and its management. Material and Methods:7 year old female child diagnosed case of sporadic Goldenhar syndrome with micrognathia, microtia, hearing loss and delayed speech. She had clubfoot deformity also. She underwent multiple surgical interventions i.e, bone assisted hearing aid implantation, limbal dermoid excision , adenoidectomy, myringotomy and correction of clubfoot. She was found to have ASD on further evaluation. Device closure could not be done due to inadequate rims of ASD., So came tous for surgical repair of large Ostium Secundum type of ASD. After her thorough preoperative evaluation, in view of difficult airway, bronchoscopic assisted intubation was done. $2.1 \mathrm{~cm}$ sized ASD was noted and closed with treated pericardial patch. Her post operative period was uneventful. Discharged on 4thPOD on ecosprin, antibiotic coverage and analgesia with vitamin supplementation. Data was collected from the Inpatient records of our hospital, o.p follow up and discharge summaries of the previous surgeries. Result: ASD closure was done with treated pericardial patch. She was discharged on 4thpost operative day with stable haemodynamics. Post operative period was uneventful. Conclusion: The first arch syndrome syndrome is associated with 1 in 10000 with congenital heart defects, of which Fallots tetrology, VSD account for most of the cases. ASD is rare (1 in 400). These patients have a good life expectancy with no major life threatening defects, so the timely correction of Heart defects if present will help improve the overall outcome in such cases. As the airwaymanagement is important for us in view of the long duration of surgery, it is challenging here due to the skeletal defects. Proper pre-op evaluation including radiological assessment of skeletal system to plan the airway management may help in decreasing their perioperative morbidity and an overall decrease in Disability Associated Life Years (DALY) . 
CHANGING SCENARIOS IN

CURRENT PRACTICE OF CORONARY

ARTERY BYPASS GRAFTING SURGERY

Arun Singh R, Sunil Agrawal, K M Cherian,

Rahul Sharma, Rakhi K R, Nikhil George, Alladi Srujan, Rajeev

St. Gregorios cardiovascular centre, Parumala, Kerala

\section{Introduction}

Diffusely diseased coronary artery is a surgical challenge in the current practice of coronary artery bypass grafting surgery. Quite often it is impossible to make conventional anastomosisin these cases until and unless we make long arteriotomy and or endarterectomy. We present our experience with this combined approach in dealing with diffusely diseased arteries.

Materials and methods

Between January and December 2018, among 258 CABG patients, 91 patients $(35.27 \%)$ had long arteriotomy with or without endartrectomy. 89 patients $(97.80 \%)$ underwent CABG using CPB other two(n=2) had off pump CABG. Median CPB time is 105 minutes. Median aorticcross clamp time is 64 minutes. Mean number of grafts is 3.68, with long arteriotomyof LAD $(n=58), \operatorname{RCA}(n=28)$, PDA $(n=16)$ and OM $(n=19)$. Results

39 patients had only long arteriotomy with patch plasty. Rest all $(n=52)$ had associated endartrectomy. Endartrectomy for LAD ( $n=25), \operatorname{RCA}(n=$ $9), \operatorname{PDA}(n=3), \operatorname{OM}(n=8)$ were done. Arteriotomy length was in range of $2.5 \mathrm{~cm}$ to $6 \mathrm{~cm}$. There was no in hospital mortality. 3rd month follow up was $100 \%$. In follow up echo no patient had decrease in ejection fraction, further 6 patients with severe LV dysfunction showed improvement in LV function

Conclusion

Long arteriotomy with or without endartrectomy is a safe and viable option in diffusely diseased vessels needing complete revascularization.

\section{PEDICLED OR SKELETONISED INTERNAL MAMMARY ARTERY IN ELECTIVE CORONARY ARTERY BYPASS?} A SYSTEMATIC REVIEW AND META-ANALYSIS.

Andrew Selvaraj, Amer Harky, Peter Eriksen, Nina Oguamana, Beverly MacCarthy-Ofosu, Andrew D Muir

1] Department of Cardiothoracic Surgery, Liverpool Heart and Chest Hospital, Liverpool, UK, 2] Barts Heart Centre, St Bartholomew's Hospital, London, UK

Objectives:We sought to compare the clinical outcomes of harvesting internal mammary artery through pedicled or skeletonised approach, in patients undergoing elective CABG.

Methods: Electronicdatabase search was performed from inception to July 2019. Only articles that directly compared the outcomes using both techniques were included. Primary outcome was Sternal wound infection. Secondary outcomes were 30-day mortality and post anastomosis flow rate.

Results: Nineteen articles met the inclusion criteria. A total of 8,567 patients were included.

Total of 4,494 patients were in pedicled group and $n=4,073$ were in skeletonised IMA group. There were no differences in preoperative patient demographics of hypertension, COPD except diabetes mellitus which was higher in skeletonised cohort $(23 \%$ vs $17 \%, 95 \%$ CI 0.77 $[0.61,0.97], \mathrm{p}=0.03$. There was no difference in the rate of using LIMA, RIMA or BIMA in either techniques. Skeletonised IMA were longer in length when compared to pedicled $(18 \hat{\mathrm{A}} \pm 3.1 \mathrm{~cm}$ vs $15 \hat{\mathrm{A}} \pm 2.3$, 95\% CI -2.37 [-3.57, -1.17], $\mathrm{p}=0.0001$ ).

Sternal wound infection was much lower in skeletonised IMA (3.5\% vs $2 \%$, $95 \%$ CI 1.95 [1.36, 2.78], $\mathrm{p}=0.0002$ ). New onset of acute MI and 30-day mortality rate were equal inboth cohorts (p\&gt;0.05). The postanastomosis flow rate was higher in skeletonised IMA over pedicled IMA ( $51 \hat{\mathrm{A}} \pm 16$ vs $39 \hat{\mathrm{A}} \pm 12 \mathrm{mls} / \mathrm{min}, 95 \% \mathrm{CI}-11.51$ [-20.54, -2.49], $\mathrm{p}=0.01$ ).
Conclusion: Harvesting IMA with skeletonised technique is associated with lower SWI rates and higher post-anastomosis flow rate. However, there are significant confounding factors and heterogeneity in the included studies, therefore the results should be interpreted carefully.

\section{AUTOMATED UNIVERSAL RECONSTRUCTION OF THE AORTIC VALVE (AURA): A NOVEL CONCEPT FOR AORTIC VALVE REPAIR}

Dr Srikant Mohapatra

Health world Hospitals, Durgapur (West Bengal)

Aim/ Objective: To develop a reliable, customizable, easily reproducible and functional solution for aortic valve reconstruction, that is universally accessible, affordable and adaptable for all circumstances.

Material\& methods: AURA relies on a cloud-based algorithm (patent pending) to virtually recreate a functional replica of the patient's normal aortic valveusing dimensional data acquired from dynamic imaging (ECHO/CT/MRI) of thediseased valve. The customized "neovalve" is then $3 \mathrm{D}$ printed in real time using pericardium or other suitable biocompatible material for aortic valve reconstruction. The process involves: $\hat{A} \cdot-$ A smart data ingestion engine to pick relevant dimensional parameters automatically by image linking. $\hat{A}$ - -Arules engine to recreate an exact virtual replica of the normal valve by extrapolating imaging data. $\hat{A} \cdot-A$ virtual simulator for hemodynamic \& stress testing of the neovalve for functional compatibility. $\hat{A} \cdot-A 3 D$ printing module for customized valve production in situ (in OT). $\hat{A} \cdot-A n$ app-based 'learn-as-you-go' smart user interface with machine learning capabilities to process data feedback from post-op imaging for further refinement. Result The proof of concept of the AURA modelling is presented in detail with validation data. Conclusion AURA fills in the demand gap for a reliable method of aortic valve reconstruction with the advantage offreedom from the limitation imposed by sizers/templates, and applicability for any age/bodyweight. The solution is also rendered future-ready with scope for minimally invasive device mounted delivery techniques \& full-scale tissue bio printing.

\section{OUR EXPERIENCE WITH}

\section{EN-BLOCK ROTATION OF THE}

OUTFLOW TRACT (EROT)

Dr. Nikhil Bansal, Lakshmi Kumari Sankhyan,

Sujoy Chatterjee, Rajarshi Ghosh,

Santosh Pandey, Srirup Chatterjee, Satyajit Bose

The Mission Hospital, Durgapur

\section{OBJECTIVE}

Double outlet right ventricle (DORV) and Transposition of great arteries (d-TGA) with anon-routable VSD constitutes a subset of patients with difficult management options. These patients in case of having two functioning ventricles should be treated with biventricular repair. EROT enables one to bring the aortic root over the LVOT, and the pulmonary root over the RVOT. Yamagishi and coworkers first described it in 2003. We present our experience with this procedure in 3 patients.

MATERIAL ANDMETHODS

Since 2019, we have performed the procedure in three consecutive patients in The Mission Hospital, Durgapur. One patient had d-TGA with non-routable VSD,PS; another had DORV with non-routable VSD,PS and the third had DORV with a non-routable VSD.

RESULTS of patients survived the procedure. Average hospital stay was 17 days. None of the three patients have a gradient in the left or right ventricular outflow tract in the follow up period and are in sinus rhythm. CONCLUSION

To date, our results with this procedure are very encouraging. In the subset of patients with DORV/d-TGA with a non-routable VSD, nd two functioning ventricles, biventricular repair forms an ideal choice. EROT 
is a suitable option in these patients as it also preserves both the outflow tracts with growth potential and linear flow dynamics.

\section{A NOVEL CALCULATION OF PRIMING VOLUME AND REQUIRED PACKED CELL TRANSFUSION TO MAINTAIN OPTIMAL HAEMATOCRIT DURING CARDIO PULMONARY BYPASS}

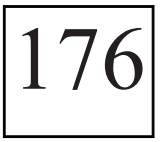

Dr.K.J.Vignesvaran, Dr. B.Karthikeyan

1. Senior Resident, Department of Cardiovascular and Thoracic Surgery, Madurai Medical College, Madurai

INTRODUCTION Hemodilution during CPB is an acceptable method to avoid the complications of continuous flow at micro circulatory level. In CPB the nadir hematocrit can vary widely with body size and pre bypass hematocrit variations, yet its effects on perioperative organ dysfunction and patient outcomes remain largely unknown. AIM This is a Prospective controlled study to identify the effectiveness of a novel formula customized in our institution for calculating priming volume and Required packed cell volume to maintain optimal hematocrit level during CPB. METHODS This study was done on 150 patients who underwent open heart surgeries in our Institute. In Group A-75 patients we have used our old protocol for calculating priming volume and the nadir hematocrit (Hct) during CPB and postoperative outcome monitored. In Group B75 patients our customized novel formula was applied for calculating PV and the nadir Hct during CPB and postoperative outcome monitored and the both the groups compared. RESULTS In our study results showed that maintaining optimal Hct of $25 \%$ (\&gt;20\%) in adult by using our novel customized formula for calculating prime volume was significantly improved the nadir Hct from $19.16 \%$ to $21.8 \%$. Complications of Hemodilution were significantly reduced. Mortality was reduced to $2.7 \%$ from $5.3 \%$. Respiratory complication reduced from $34.7 \%$ to $17 \%$. Coagulopathy reduced from $20 \%$ to $7 \%$. CONCLUSION Our Study showed that, application of this novel formula is very useful and easy in maintaining optimal Hct during $\mathrm{CPB}$ thereby improving the postoperative results Keywords: Hemodilution, Optimal Hematocrit, priming volume, Required packed cell volume.

\section{INFECTED CORONARY STENT- A NIGHTMARE}

Jeevesh John Thomas, Bhaskar Ranganathan,

Simon Philipose, Shylesh Kumar, Jose Chacko

Periapuram

Lisie Heart Institute, Lisie Hospital, Kaloor, Kochi, Kerala

\section{BACKGROUND}

Coronary stent infection is a rare condition and reported data is still less. It is however associated with a mortality rate of about $50 \%$. Medical management is the first line of management but it is often difficult to eradicate the infection and surgery is needed in these cases. Surgery is also needed for complications that arise from infected stents such as perforation, tamponade, coronary cameral fistulas and aneurysm formation.

METHOD

We herein describe the case of a 45 year old lady who underwent stenting of the proximal LAD (Left Anterior Descending) at a public hospital on $12 / 06 / 19$. She was readmitted there with fever and chest pain and a check angio showed patent stent. She came to our hospital after 1 month with further episodes of fever and chest pain. She had large pericardial effusion on echo. A CT chest revealed moderate pericardial collection, diffuse thickening of pericardium and loculations around the stent. The pericardial aspirate grew Pseudomonas on culture and she was started on Meropenem. A diagnosis of infected stent was made. Despite 3 days of antibiotics, her fever persisted and hence she was taken up for surgery. A coronary shoot showed aneurismal changes surrounding the LAD stent. Per operatively, extensive adhesions were found and anabscess cavity was seen around proximal LAD with pus within. Cardiopulmonary bypass was established with aortic and bicaval canulation. During further dissection, the abscess burst open and the stent was extruded. After arresting the heart, pulmonary artery was divided to expose the proximal LAD. The proximal LAD was closed off with pledgetted sutures on either side. LIMA was harvested and anastomosed to the distal LAD. Weaning from bypass was difficult and neededIntra Aortic Balloon Pump support. In view of extensive bleeding from the raw surfaces, the chest was left open with packs and a delayed closure was done after 24 hours. Tissue culture from the abscess wall also grew Pseudomonas and she wax continued on antibiotics for 4 weeks. She is now well recovered and stable cardiac wise. CONCLUSION

Infections of coronary stents is a dreaded though rare condition. Drug eluting stents are at higher risks in view of impairment of local defense mechanism. An infected coronary stent is a challenge for surgeons and needs diligent surgery for a successful outcome.

\section{CHRONIC GRANULOMATOUS PULMONARY ASPERGILLOUS MASS IN AN IMMUNOCOMPETENT PATIENT- RARE FIRST CASE REPORT \\ Dr.Sreekar Balasundaram, Dr. Jayanthi Savio, Dr. Uma Maheshwari, Dr. Priya Ramachandran, Dr. Uma Devaraj, Dr. Kavitha, Dr. Suresh Kumar St.John's Medical College Hospital, Bangalore}

1)AIM/OBJECTIVE: To present a rare case of a Pulmonary Aspergillus mass with features of chronic granulomatous infection in an immunocompetent patient, not reported in the literature previously.2) MATERIAL AND METHOD:A 34-year-old male presented with a history of rightsided chest pain and streaky hemoptysis for one month. 8 months ago, he was found to have an incidental right lung mass on Chest X-Ray on routine health check-up. He refused further evaluation until he became symptomatic. A CT guided biopsy revealed granulomatous inflammation with tissue cultures positive for septate fungal hyphae. He was otherwise fit with good exercise tolerance, seronegative and no previous tuberculosis. Interesting operative data, gross pathology, and histopathology of this rare presentation of Pulmonary Aspergillus infection will be presented. Data from the patient and his records, regarding history, symptoms, past history, laboratory and culture reports, operative findings and postoperative histopathology and other special investigations, were compiled. 3)RESULT: He underwent a successful Right pneumonectomy with partial resection of the right $3 \mathrm{rd}$ and 4 th ribs. He has been doing well on 6 months follow up.4) CONCLUSION: A unique and rare presentation of Pulmonary Aspergillus Infection and its management has been presented. Challenges to Clinical diagnosis, differential diagnosis, Investigations and management that should comprise a team of Radiologists, Pulmonologists and Microbiologists with experience in Fungal infections are emphasized.

Rheumatic mitral stenosis simulating double-orifice mitral valve

Dr Ritesh Mate, Dr. Prashant Mishra, Dr Jayant Khandekar, Dr. Chaitanya Raut. Department of C.V.T.S., LTMMC \&

LTMGH, Sion, Mumbai, India

Description A 26-year-old woman presented to us with symptoms of breathlessness New York Heart Association class III for 6â€ ...months on and off with a history of rheumatic fever in childhood. She was diagnosed in the periphery as having rheumatic heart disease for 8 â€ ...years. The patient was on regular injectable penicillin prophylaxis (injection penicillin $1.2 \hat{a} €$...million units every 21 st day), $\hat{\mathrm{I}}^{2}$-blockers with intermittent diuretics. Cardiac examination revealed low pitched mid-diastolic rumble grade IV/VI at apex with normal S1. Twelve-lead ECG showed 
atrial fibrillation with controlled ventricular rate. Echocardiogram revealed severe mitral stenosis with severe subvalvular disease. Severe subvalvular fusion created double orifice appearance (figures 1) The combined mitral valve area of two orifices was approximately $1 \mathrm{~cm} 2$ (figure 3), gradient was 17/11â€ ...mmâ€ ...Hg .The patient underwent mitral valve replacement with mechanical bileaflet valve. She is doing well on follow-up. Discussion : Double orifice mitral valve (DOMV) is a rare congenital anomaly of the subvalvar mitral valve apparatus (chordae tendinae and papillary muscles) consisting of an accessory bridge of fibrous tissue, which partially or completely divides the mitral valve into two orifices. The size of the orifices is balanced in only $15 \%$. Anatomically and functionally, the mitral leaflets are essentially normal in most cases, but they can be regurgitant or stenotic. DOMV rarely occurs as an isolated anomaly, but is most commonly associated with a variety of other cardiac anomalies such as atrioventricular septal defects, coarctation of the aorta, bicuspid aortic valve, sinus venosus atrial septal defect, ventricular septal defect, patent ductus arteriosus, hypoplastic left heart syndrome, double orifice tricuspid valve, tetralogy of Fallot, and Ebstein's anomaly. Acquired DOMV may be present after surgical correction of a mitral valve prolapse where the free edge of the middle portion of the prolapsing anterior or posterior leaflet is anchored to the facing edge of the opposite leaflet (â€œedge to edgeâ€ or â€œdouble orifice repairâ€ technique described by Alfieri). Differentiating features were history of rheumatic fever in childhood, thickened valve leaflets, severe subvalvular disease with existence of two papillary muscles.

\section{AORTIC DISSECTION WITH MULTI ORGAN INVOLVEMENT \\ Jayaprakash, Mariappan \\ Institute of Cardiothoracic surgery, \\ Madras Medical College}

Aortic dissection is a rare life threatening condition. A very high index of clinical suspicion is necessary for the accurate and rapid diagnosis of aortic dissection. We report a case of a 38 years old male known case of chronic kidney disease and Hypertension who presented with signs and symptoms of myocardial infarction and one valuation found to have aortic dissection and was successfully managed with surgery

Case report:

A 38 year old male with history of chronic kidney disease, hypertension presented with chest pain, left upper limb weakness and transient loss of consciousness. Blood reports with elevated renal parameters. Echocardiography shows hypokinesia of inferior wall with Disssection flap noted in ascending aorta extending into arch of aorta and mild AR. Coronary Angiography revealed Acute Type A Aortic dissection and Occluded RCA arising from false lumen. CECT Aortogram confirms the diagnosis with dissection involving root of aorta, ascending aorta and arch ofaorta with thrombus in right coronary artery. MRI Brain with MRA \&amp; MRVrevealed infarct in right temporo parietal, splenium of corpus callosuminfarct and chronic hemorrhage in right ganglio capsular infarct. The patient underwent Ascending aortoplasty with Interposition (Dacron) graft and Coronary artery bypass grafting to mid RCA. Diffuse Bleeding in posterior part of the graft noticed and was packed with gauze roll. Tip of the gauze roll was brought out from the edge of the skin wound near lower end. He was stabilized with multiple inotrophes and closely monitored in ICU and extubated after $24 \mathrm{hrs}$. On 3rd Post operative day under ivsedation gauze roll removed and no active bleeding or drain noted. Hemodialysis was done post operatively for elevated renal parameters and hyperkalemia. He improved well. Post op Echo revealed no dissection flap in aortic root, ascending aorta and arch and discharged on 15th Post operative day. He is on regular follow up and renal parameters improved and left hand weakness improved Conclusion:

We report this case for unusual mode of presentation of Aortic dissection along with acute kidney injury, RCA territory infarct and cerebralinfarct and managing the intra operative diffuse bleed and optimizing the renal parameters

\section{LATE PRESENTING AORTOPULMONARY WINDOW AN UN COMMON LESION ASYMPTAMATIC UNTIL THE AGE OF 23 YEARS \\ DR. Kalyan Babu. DR. R V. Kumar, \\ DR. Amarash, DR. Ramakrishna, \\ DR Sai Surabhi, DR. Gopal \\ Nijams Institute of Medical Sciences Hyderabad}

Aorto pulmonary window a rare congenital macrovascular malformation constitutes $\& 1 t ; 1 \%$ of congenital heart disease. This case involve a 23years male complaining of sob 11l, chest pain, occational blood stain expectoration,clinically pan systolic murmer, parasternal heave, 12 leeds ecg left ventricular hypertrophy, chest xray increased cardio thoracic ratio, with severe pah, cardiac catherisation base line systamic desaturation, oxygen saturation shows segnificance step up at pulmonary artery level, ctpa with $3 \mathrm{~d}$ reconstriction right pulmonary artery communicate with ascending aorta $3.8 \mathrm{~cm}$ distal to the root of aorta as septal defect type 11 . Diagnosed as aorto pulmonary window type 11 and type 11l. surgery is perform under Cardio pulmonary bypass followed by decreased pulmonary pressure. Aorta open transversly, MPA open vertically. Findings type 11 andtypelll conformed. Right pulmonaryartery arising from posterior wall of distal ascending aorta. APwindow identified, defect closed with gluteraldehyde treated pericardial patch. patient recover postoperatively with no discomfort. This rare condition that can be treate successfully appropriate pre operative diagnosis and surgical management and post operative fallow up of reducing the pulmonary vascular resistance.

\section{RHEUMATIC MITRAL STENOSIS SIMULATING DOUBLE-ORIFICE MITRAL VALVE.}

Dr Ritesh Mate, Dr. Prashant Mishra,

Dr Jayant Khandekar, Dr. Chaitanya Raut

Lokmanya Tilak Municipal General Hospital and Lokmanya Tilak Municipal Medical College, Sion Mumbai

Description: A 26 year old woman presented to us with symptoms of breathlessness NYHA class III for $6 . .$. months on and off with a history of rheumatic fever in childhood. She was diagnosed as having rheumatic heart disease for $8 \ldots$ yrs. Examination revealed low pitched mid-diastolic rumble grade IV/VI at apex with loud S1. 12 lead ECG showed atrial fibrillation with controlled ventricular rate. Echo revealed severe mitral stenosis with severe subvalvular disease. Severe subvalvularfusion created double orifice appearance (DOMV). The patient underwent mitralvalve replacement with mechanical bileaflet valve.

Discussion: DOMV was first described by Greenfield in 1876 . This anomaly is characterized by the presence of a single mitral annulus with two orifices, each having independent subvalvular apparatus and papillary muscles. DOMV rarely occurs as an isolated anomaly, but is most commonly associated with a variety of other cardiac anomalies. The subvalvar mitral valve apparatus consisting of an accessory bridge of fibrous tissue, which partially or completely divides the mitralvalve into two orifices. Three specific types of DOMV are describe basedon 2D Echo complete bridge type (about $15 \%$ of DOMVs), Incomplete, bridge \&amp; holetypes. Anatomically and functionally, the mitral leaflets are essentially normal in most cases, but they can be regurgitant or stenotic. Acquired DOMV may be present after Rheumatic affection of mitral valve, surgical correction of amitral valve prolapse (Alfieri Stitches). Differentiating features were historyof rheumatic fever in childhood, thickened valve leaflets, severe subvalvulardisease with existence of two papillary muscles. 


\section{MECHANICAL SUPPORT IN}

THE PERI TRANSPLANT PERIOD OUTCOMES

Jeevesh John Thomas, Bhaskar Ranganathan,

Simon Philipose, Manoras Mathew C, Shylesh Kumar,

Jacob Abraham, Job Wilson P, Grace Maria George,

Antony George, Jose Chacko Periapuram

Lisie Heart Institute, Lisie Hospital, Kaloor, Kochi, Kerala

\section{AIMS and OBJECTIVES}

Hemodynamic instability inorthotropic heart transplant $(\mathrm{OHT})$ recipients in the peri-transplant period is deleterious and not infrequent. Mechanical circulatory support (MCS) devices have been used to provide temporary hemodynamic support. We aim to study the use of MCS in our transplant patients before and after cardiac transplantation.

METHODS

We did 23 orthotopic heart transplants in the period of 2013 to 2018 . The use of MCS for these patients pre or post procedure was studied. The support systems used were Intra Aortic Balloon Pump (IABP), Extra Corporeal Mechanical Oxygenation (ECMO), and Bi Ventricular Assist Device (BiVAD). The post operative outcome in the immediate, early and late follow up was studied on a case by case basis.

RESULTS

7 out of the 23 patients required some form of mechanical support in the peri transplant period. One patient required ECMO support which was later converted to a BiVAD in the preoperative period. 3 patients required ECMO in the immediate post operative period. They had IABP support in addition for a variable period of time. 3 patients required IABP support alone in the initial 48 hours after transplant. All the postoperative MCS were considered to be related to primary graft failure, however, hyper acute rejection could not be ruled out completely.

The patient who had MCSpre operatively had a transplant in 14 days after BiVAD insertion and was put on IABP support in the immediate post operative period from which he was successfully weaned. His 3 year outcome is good. Patients who needed IABP alone were also doing well in their early and mid term follow up. 3 who were on post transplant ECMO did not have good outcomes with 2 of them dying in a week and one who was successfully weaned off ECMO died of infection 30 days after transplant.

CONCLUSION

In summary, primary graft failure after heart transplantation continues to be associated with important morbidity and mortality. In patients who are refractory to medical management, early institution of MCS leads to improved outcomes.

\section{TOTAL VERSUS PARTIAL}

\section{CHORDAL PRESERVATION IN}

MITRAL VALVE REPLACEMENT:

EARLY RESULTS

DR Sistla VPL Chandrakumar,

DR Sunil Dhar, DR Madhur Kumar,

DR Ajit Kumar Padhy, DR Anubhav Gupta

Vardhman Mahavir Medical College and Safdarjung Hospital, Delhi

\section{Introduction}

Not conserving the chordae during MVR (Mitral Valve Replacement) results in loss of normal torsional deformation of the apex there by disrupting the stress- strain pattern of the Left Ventricle. We compared the early outcomes of chordal preservation of patients who underwent MVR at our institute.

Materials and methods

168 patients underwent MVR from January 2016 to January 2018 at VMMC and SJH (VARDHMAN MAHAVIR MEDICAL COLLEGE AND SAFDARJUNG HOSPITAL), Delhi. We compared the outcomes of those who underwent MVR with Total preservation of chords $(n=44)$ with thosein whom the chords of the PML (Posterior Mitral Leaflet) alone were preserved $(n=87)$ and those in whomall the chords were sacrificed $(n=37)$. Patients who underwent MVR along withaortic valve procedures, CABG (coronary artery bypass grafting), by MICS (Minimally Invasive Cardiac surgery) were excluded from the study.

Results

Of the 168 patients, 44 patients underwent total chordal preservation. There was a significant reduction in the LVes (Left Ventricular EndSystolic Dimensions) and LVed (Left Ventricular End-diastolic imensions) at six-month follow up in those patients who underwent total chordal preservation. Even though Left ventricles of patients who underwent partial or nochordal preservation also regressed, the change was marked in those with total chordal preservation. There were no incidents of LVOTO (Left ventricular Outflow Tract Obstruction) in our series. We advocate routine total chordal preservation during mitral valve replacement.

\section{PERI-OPERATIVE AND MIDTERM RESULTS OF HYBRID PROCEDURE FOR COMPLEX AORTIC}

\section{PATHOLOGIES: OUR EXPERIENCES}

Dr. Noaman K Shaikh, Dr. Arnab De, Dr. Tejas Dandekar, Dr. Vaibhav Shah, Dr. Chaitanya Raut, Dr. Prashant Mishra, Dr. Jayant V Khandekar Lokmanya Tilak Municipal Medical College and Hospital, Sion, Mumbai.

Aim: To evaluate the peri-operative and midterm(6months post surgery) outcomes of patients undergoing Hybrid Procedure for complex aortic pathologies. Method: A total of 8 patients who underwent Hybrid Procedure for various complex aortic pathologies from January 2014 to May 2019 were analyzed retrospectively. All patients were subjected to a single-staged procedure by the same team of Cardio-Vascular and Thoracic surgeons and interventional radiologists, in a hybrid operation theatre set-up. Case records on 6 month follow-up which included a CT aortogram were also reviewed. Results: The patients with a mean age of 49.6+/-8.89years, with 5 patients with type A aortic dissection, 2 type B aortic dissection and 1 case of aortic arch aneurysm. Of the 8 patients operated, 1 developed new onset renal insufficiency in the immediate post-surgery period and succumbed on post-op day 6 . Of the remaining patients 1 patient was noted to have type $1 \mathrm{~b}$ endo leak on 6 month followup and was subjected to a successful distal extention. No late extraanatomic bypass occlusions or stent graft migrations were appreciated on follow-up CT scans. None of the patients developed paraplegia or stroke. Conclusion: The above study demonstrated acceptable results in patients subjected to hybrid procedures for complex aortic pathologies at our institution.

\section{INDIA'S LARGEST CARDIAC TUMOR SUCCESSFUL EXCISION EXPERIENCE IN MADURAI MEDICAL COLLEGE}

Dr. A.S. MUGHILAN, Meenakshi sundaram MS MCh, Muthukumar M MS MCh, Rathinavel MS MCh Department of Cardiovascular Thoracic Surgery, Madurai Medical College

Introduction:

Primary cardiac tumors are extremely rare, with a reported incidence ranging from 0.0017 to $0.019 \%$. Of such tumors, about $75 \%$ are benign nature. Cardiac fibromais the second most common primary cardiac tumor in infants and young children after rhabdomyoma. Although cardiac fibroma is a benign and solitary tumor - composedof fibroblasts and collagen, it is clinically important as it may present with symptoms such as inflow and outflow obstruction, conduction system disease, and 
sudden death. In contrast to cardiac rhabdomyoma, cardiac fibroma rarely regresses spontaneously and surgical removal in a symptomatic case should be considered.

Case report:

Here we report a case of 11-year-old girl presented with dyspnea on exertion 2 months. Echo-cardiogram and CECT imaging indicated a large mass located in the right ventricular wall. The patient underwent Complete surgical excision of ventricular tumor with Glen shunt and histopathological examination confirmed the mass to be a fibroma. The patient had a good postoperative recovery and was discharged on POD 8. Result:

Right ventricular cardiac tumor excised successfully with Glen shunt in order to reduce RV dysfunction post operatively. Post operatively patient recovery was good, and post-operative echo was uneventful. Conclusion:

From the literature reviewed throughout India, patient we operated was found to be the largest cardiac tumor in India.

\section{BIVENTRICULAR OUTFLOW TRACT OBSTRUCTION WITH VENTRICULAR SEPTAL DEFECT: A CASE REPORT}

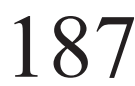

Dr. Mohammad Hafiz, Dr. Chandraprakash Srivastava, Dr. Saify Arsiwala, Dr, Ankit Mathur, Dr, Prashant Mahawar

Narayana Multispeciality Hospital, Jaipur

Biventricular outflow tractobstruction with ventricular septal defect is a rare anomaly. Primary repair for biventricular outflow tract obstruction associated with ventricular septal defect is till now very challenging. The outcomes \&amp; risk factors are lesss tudied.

We report a case of 21/2year old Indian female who came to our hospital with shortness of breath on exertion \&amp; poor weight gain, evaluated and diagnosed to have situsin versus, dextrocardia, L-looped ventricles, inversely related great arteries, large sub aortic ventricular septal defect, severe pulmonary stenosis, moderatemitral regurgitation, severe sub aortic stenosis, inferior vena cavainterruption, right arch, bilateral superior vena cava, right superior venacava draining to coronary sinus. Patient underwent successful intracardiacrepair including sub aortic ventricular septal defect closure, pulmonaryvalvular commissurotomy \&amp; subaortic membrane resection.

Primary repair of biventricular outflow tract obstruction associated with ventricular septal defect provide good outcomes. Selective \&amp; adequate resection of outlet obstruction with complete closure of ventricular septal defect is the main factor for successful repair with avoidance of heart block.

\section{UNWANTED COMPLICATION} DURING LEFT ATRIAL MYXOMA SURGERY Dr Rakesh Kumar, Dr Rani Singh

Rajendra Institute of Medical Sciences (RIMS) Ranchi

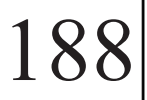

Aim- To highlight what is not required during Left atrial myxoma surgery Material and methods 47 years old famle patient having difficulty in breathing for last 4 months, got incidental diagnosis of left atrial myxoma with suspectal clot in left atrial appendage. While inspecting left atrial appendage during surgery with Russian forcep there caused rent in left atrial appendage. While coming off cardiopulmonary bypass we noticed it. Result-The rent in left artrial appendage repaired and secured hemostasis by externally ligating the left atrial appendage. Conclusion-1. Always inspect behind pulmonary artery if any manuvre done in left atrium or left atrial appendage, before comoing off bypass or at least before decannulation.2. Dont become panic in untoward circumstances to avoid unnecessary chaos in OT.
IMPACT OF PRE- OP USAGE

OF CLOPIDOGREL ON CLINICAL OUTCOME OF OPCAB PATIENTS

Ajay Kumar Thangaraj,Sanjay Theodore

Chettinad Hospital and Research Institute

\section{BACKGROUND}

The usage of clopidogrel and aspirin as the dual anti- platelet therapy is to reduce the risk of thrombotic events in patients with Coronary artery disease. In this study we have compared the impact of pre- op usage of clopidogrel in the patients who underwent OPCAB

MATERIAL AND METHODS

We have taken 950 patients whounderwent $\mathrm{OPCAB}$ in our institute in the period of 5 years (2014 August to 2019October). Group A- patients who have not stopped DAPT 5 days prior to surgery. Group B- patients who stopped Clopidogrel 5 days prior to surgery. Aspirin was continued in patients of both groups. Final results were concluded by the postoperative parameters such as drain, requirement of blood transfusion, re-exploration, ventilatory hours, ICU stay, hospital stay, morbidity and mortality rate.

\section{RESULT}

Group A patients had higher rate of post- operative bleeding (p-0.049) but blood transfusion rate was notstatistically significant $(\mathrm{p}-0.91)$. There was a significant drop in post op Hbin group A patients with no statistical significance (p-0.83). The mean ICU/hospital stay were less in group B patients (p-0.17,0.19 respectively).

\section{CONCLUSION}

The usage of DAPT prior to the surgery does not have significant impact on the early outcomes of OPCAB patients except for the duration of hospital stay. Hence it is safe to continuethe usage of Dual Anti-platelet therapy prior to surgery.

\section{VANCOMYCIN LOCAL APPLICATION, ROLE IN DEEP STERNAL WOUND INFECTION (DSWI) IN OUR EXPERIENCE. Manish Jadhao, Chaitanya Raut, \\ Vaibhav Shah, Prashant Mishra, \\ Jayant Khandekar, Kuntal Surana \\ Lokmanya Tilak Municipal Medical College and \\ General Hospital}

Introduction: We aim to study the role of vancomycin paste applied locally over sternal edges in reducing Deep sternal wound infection (DSWI).

Materials and Methods: We retrospectively collected data of patients who underwent open heart surgery from June 2017 to May 2019 in our institution. The rate of DSWI was compared in patients in whom vancomycin paste was applied and not applied. Vancomycin paste was applied just after sternotomy and at the time of sternal closure. The rate of DSWI was also compared in the following high risk sub groups: Age \&gt; 65 years, female patients, diabetes, morbidly obese (BMI\&gt;30) and chronic obstructive lung diseases.

Results:

A total of 862 patients underwent open heart surgery during the specified period. Overall incidence of DSWI in our study was $3.48 \%$. DSWI was found to be significantly lower in the vancomycin group $(1.86 \%$ vs. $5.09 \% ; \mathrm{p}=0.0144)$. DSWI in diabetic patients was also significantly lower in the vancomycin group $(1.36 \% ; \mathrm{v} / \mathrm{s} 7 \% \mathrm{p}=0.033)$. Overall incidence of DSWI in COPD patients was $(13.41 \%)$ in our study, and it was significantly lower in the vancomycin groups compared to non vancomycin group $(5 \% \mathrm{v} / \mathrm{s} 21.42 \% ; \mathrm{p}=0.0489)$. No deaths were recorded. Conclusion: The use of local vancomycin paste application is recommended especially in CABG patients and those with co morbidities like diabetes mellitus, COPD, morbidly obese (BMI\&gt;30) with diabetes mellitus, old age and female patients. 
SUCCESSFUL REPAIR OF TRANSECTED COMMON CAROTID ARTERY

Dr Suresh Babu Kale, Dr. Punithakumar Ramasamy, Dr Chennakesavulu

Meenakshi Hospital, Thanjvur, Tamilnadu State, India.

\section{Background}

Penetrating neck injuries often involve the common carotid artery and various degree of injury is described. Transection of the carotid vascular tree is grievous, leading to hypovolemic shock, stroke and ultimately death.

Methods

A 26years old female patient presented with hypovolemic shock after being stabbed in the left side of neck. The pulse was feeble, extremities cold and blood pressure 60/40 millimeters of mercury. Immediate tracheal intubation was done and volume replaced. Computed tomographic angiogram of the head and neck vessels showed completed transection of the carotid vascular tree with clots. Immediate repair was done with $6 \mathrm{~mm}$ interposition PTFE grafting of the divided artery, direct repair of the internal jugular vein and direct repair of the vagal trunk. Cerebral protection was achieved with cooling the head with ice bags, hyperventilation and steroids.

Results

There was no operative mortality or major morbidity. The patient was ventilated overnight and extubated successfully the next day morning. The patient was able to ambulate on day 2 and was transferred from the intensive care unit on day3.No neurological deficits were seen. Conclusions

Rapid investigation and quick repair of the common carotid transection saves life and may avoid major stroke. Cerebral protection measures must be employed in all these cases.

\section{RARE CASE OF PATIENT}

LARGE SUBAORTIC VSD WITH

SUBAORTIC MEMBRANE

Dr Rakesh Kumar ,Dr Rani Singh

Rajendra Institute of Medical Sciences Ranchi

Aim-An unusual combination of large subaortic VSD with subaortic membrane. Material and methods-A 3 yrs old male child having history of recurrent LRTI and failure to thrive with delayed milestones. Further investigation done and found to have the VSD with SAM. ResultSuccessfully clsed VSD with patch and SAM resected, post resection there were no residual SAM. post op period was uneventful. Conclusion-Meticulous resection of SAM is compulsory and the VSD patch should sufficiently cover the VSD and the surures should be place meticulous and preferably interrupted sutures for VSD closure.

\section{ASSESMENT OF PULMONARY \\ HYPERTENSION CHANGES IN PATIENTS \\ UNDERGOING MITRAL VALVE REPLACEMENT AND CORRELATION WITH HEATH EDWARDS PATHOLOGICAL CHANGES IN LUNG PARENCHYMA. \\ Vipin B Nair, Abdul Rasheed \\ Government medical college trivandrum}

50 cases of rheumatic mitral valve stenosis who underwent mitral valve replacement in 1 year were assesed for reduction in pulmonary hypertension using echo. Also the lung parenchymal changes in these patients were graded as per heath edward pathological grading system. The analysis involved the correlation between preoperative and postoperative pulmonary hypertension and validation of heath edward grading in all these patients
DO WE UNDERTAKE TOO MANY

BLOOD TESTS AFTER CARDIAC SURGERY

Dr Sobaran Singh Sharma, Yasir Ahmed,

Joseph George, Umair Aslam, Aprim Youhana,

Pankaj Kumar

Morriston Hospital, Swansea, U.K.

Objectives Post-operative cardiac surgical patients undergo intensive monitoring, including multiple serial blood testing for arterial blood gas monitoring and routine blood panels. There is increasing recognition of the total blood utilised to undertake these blood tests. Our aim in this study is to quantify the blood loss from routine blood-testing. We also evaluate the haemoglobin concentration and transfusion requirements after cardiac surgery and its financial implications. Methods Retrospective review of prospectively-collected data for patients undergoing cardiac surgery in our unit was scrutinised. Total blood utilised to undertake tests were calculated by interrogating the patient charts and pathology database. Results306 patients were included in the study. Procedures undertaken were: isolated coronary bypass surgery 106 cases, isolated valve replacement 86 cases, aortic/combined/other procedures 114 cases. A total of 23,572 blood samples making $125.5 \mathrm{~L}$ were drawn for blood samples, averaging 77 samples and $415 \mathrm{ml}$ per patient. Blood gas analysis comprised majority of the volume drawn $(240 \mathrm{ml})$, followed by electrolytes $(53 \mathrm{mls})$. Patients haemoglobin concentration dropped by an average of $27 \mathrm{~g} / \mathrm{L}$. For the entire cohort of this group of patients an average of 2.8 units of blood were transfused at a cost of pound 128,550.conclusion Approximately 1.5 units of blood were withdrawn on average for blood testing after cardiac surgery. Blood drawn for tests may have an impact on the patient's haemoglobin concentration resulting in anaemia and need for blood transfusion. Our study is the first to report results from a European perspective and gives further insight into the potential areas where blood test volumes could be minimised.

\section{CUSTOM MADE DEVICE - A SIMPLE SURGICAL SOLUTION FOR APICAL MUSCULAR VSDS}

Mrinal Patel, Dr Vishal Agrawal, Dr Amit Mishra UNMICRC

Apical muscular VSD ( Ventricular Septal Defect) constitutes 2\% of the cardiac defects encountered in pediatric cardiac patients. However the technique for closing it is still a matter of debate. We present an innovative, cost effective and simple surgical technique using custom made PTFE ( poly tetra flouro ethylene) single disc umbrella shape device for closing multiple apical muscular and isolated apical muscular VSD. The device is easily prepared during the surgery. This technique has the advantage of apical VSD closure through leftt venticle without ventriculotomy. The procedure has a good outcome with a low complication rate.

\section{WORLD'S FIRST MULTIVESSEL TOTAL ARTERIAL CORONARY ARTERY BYPASS GRAFTING IN MESOCARDIA.Y GRAFTING IS THE WAY DUE TO ANATOMICAL REASONS. \\ Dr. Shubhi Bhatnagar, Dr. Prateek Bhatnagar \\ Beating Heart Clinic,Hyderabad}

Mesocardia is an extremely rare congenital cardiac anomaly of counterclockwise rotation of the heart. We report here a case of coronary artery bypass grafting inmesocardia, which has not been described in medical literature. A 52-year-old man had an anterior wall myocardial infarction. Chest roentgenogram, two-dimensional echocardiography, and computed tomography of the chest confirmed mesocardia. Off-pump total arterial 
revascularization was done. Computed tomography coronary angiography done 1 year after surgery showed all 4 grafts patent. Exposure of aorta and right atrium through the standard median sternotomy is very difficult in mesocardia owing to cardiac rotation and should be kept in mind in this surgery.

\section{POSTOPERATIVE ATRIAL FIBRILLATION IN CORONARY ARTERY BYPASS GRAFTING HERALD POOR OUTCOME}

Pulkit Malhotra, Supaksh Mahindru, S Pande, S K Agarwal

Sanjay Gandhi Post Graduate Institute, Lucknow

Introduction: Atrial fibrillation in postoperative period is a common incidence reported between $10 \%$ and $30 \%$. Atrial fibrillation in coronary artery disease has been attributed to the age of presentation and the disease process per say. Though, the event of atrial fibrillation and its consequences well documented, its effect on outcome is not clear.

Material and Methods: This is a retrospective study of patients operated between January 2018 to April 2019. A total of 263 patients received coronary artery bypass grafting during this period. All the patients received total arterial revascularization using two internal mammary arteries with no touch aortic technique. The data acquisition was done from hospital records and operating log register. The data for demographics, comorbidities, preoperative medications, operative details and echocardiographic parameters of left ventricular functions were acquired. A total of 24 patients had atrial fibrillation (Group I) while 239 remained in normal sinus rhythm (Group II). The outcome is measured as combined of death and postoperative MI. The two groups are compared by nonparametric tests for all the variables.

Results: The groups are comparable in demographic, preoperative medications, operative and left ventricular parameters. Of the 24(9.12\%) patients which had postoperative atrial fibrillation, 11 were discharged on medical management. Thirteen patients reverted to sinus rhythm at discharge. Electrical cardioversion was done in 8 patients and successful in 5 to achieve sinus rhythm at discharge. Medical treatment reverted 8 other patients to sinus rhythm at discharge. Atrial fibrillation was present in 8patients 1 week after discharge and 3 patients had atrial fibrillation after 1 month. The requirement of intra-aortic balloon pump was statistically significant in group I ( 5 in group I vs 10 in group II, $n=0.0001$ ). The combined end point was achieved in $8(25 \%)$ in group I vs $27(11.2 \%)$ patients in group II, $\mathrm{p}=0.10$.

Conclusion: The appearance of atrial fibrillation heraldsincreased requirement of IABP and combined end point of postoperative myocardial infarction and death in patients undergoing coronary artery bypass grafting.

\section{A CASE REPORT OF CORONARY ARTERY ANUERYSM}

Pardhasarathi Reddy Allam, RM Krishnan,

M Rajan, S Kumar, K Balamurugan

Meenakshi Mission Hospital and Research Centre

\section{Introduction}

Coronary arteryaneurysms (CAA) are not uncommon. First described by Bourgon in 1812. CAA defined as dilatation of coronary artery more than 1.5 times the diameter of adjacent segments or the diameter of patients largest coronary vessel. Overall incidence ranges from $0.3 \%$ to $5 \%$. Right coronary artery most commonly affected. Atherosclerosis is most common cause of CAA, there is increased incidence of occurrence of CAA following angioplasty.

Case Report

42 years old male non diabetic, non smoker,coronary artery disease, post angioplasty to LCX and LAD, presented with history of chest pain since 4 days on evaluation, coronary angiography revealed large coronaryaneurysm arising from proximal LAD and proximal LCX with no flow visualised beyond aneurysm. He underwent on pump CABG to LIMA to LAD,SVG to OM withcoronary anuerysm closure, mouth of the aneurysm ligated and aneurysmal sacclosed. Post operative was uneventful, he was discharged on sixth post operative day, he is doing well on follow up. Conclusion

CAA are not uncommon, in the era of increased angioplasties. CABG with repair of aneurysm should be the choice of treatment for obstructive CAA.

\section{EFFECT OF MITRAL VALVE REPLACEMENT ON PULMONARY ARTERIAL PRESSURES IN MITRAL VALVE DISEASE WITH PULMONARY HYPERTENSION-A RETROSPECTIVE}

\section{ECHOCARDIOGRAPHIC OBSERVATIONAL STUDY}

Dr Suraj Pai, Dr Durgaprasad Rath, Dr Ramsankar, Dr Hemachandren, Dr Saichandran

JIPMER, Pondicherry

Aims: To assess the change in pulmonary arterial pressure among patients who underwent mitral valve replacement Materials \&amp; methods: It was a retrospective observational study. Data of subjects with mitral stenosis and/ or mitral regurgitation with pulmonary arterial hypertension who underwent mitral valve replacement were obtained. Sampling was done with deidentification of subjects, records of 60 subjects were obtained and Echocardiography reports were reviewed. Echocardiography findings obtained were before surgery, 1 week after surgery, then at 1 month and 6 months after surgery. Results: Mean age of the subjects was 43.4 years, with majority being females. All patients had dyspnea, 45 patients had chest pain, 40 patients had palpitations. It was observed that 37 patients had preoperative atrial fibrillation. There was a significant reduction in the mean pulmonary arterial systolic and diastolic pressures, and also pulmonary vascular resistance at every stage of the recording ( with p-valve of \&lt;0.05)Conclusion: There was a significant drop in systolic and diastolic pulmonary arterial pressures along with pulmonary vascular resistance from the data obtained. Mitral valve replacement had a positive impact on pulmonary arterial pressures following surgery performed in subjects with pulmonary hypertension. This is in concordance with the research hypothesis which states that following mitral valve replacement in patients with mitral valve pathology and pulmonary hypertension, there is a decrease in pulmonary vascular resistance post operatively.

\section{MECHANICAL PROSTHETIC VALVE THROMBOSIS IN CURRENT ERA: 5 YEAR FOLLOW-UP.}

Supaksh Mahindru, Shantanu Pande

Sanjay Gandhi Post Graduate Institute of Medical Sciences

Introduction: The incidence of prosthetic valve dysfunction (stuck valve) is variable and is dependent on various factors. Apart from the nature of the mechanical valve that is thrombogenic, prothrombin time management, literacy, socioeconomic status and motivation to continue anticoagulation are some of the important factors that lead to such events. There are studies from Indian groups that have emphasised the success of thrombolysis in these cases, there are no study which have followed them for midterm. This study is designed to emphasise on the follow-up of these patients at midterm.

Material and Methods: The patients reporting to the hospital with severe dyspnoea and hemodynamic compromise who were confirmed to have prosthetic valve thrombosis on echocardiography are included in this study. The patients admitted with stuck valve between period of January 2013 and December 2017 are included in this study. Hospital record and operative logs are used to acquire the data for this study. A total 
of 66 patients were admitted with stuck valve during this period. All the patients received thrombolysis as the preferred modality of treatment. Patients included were operated between 1998 and 2017. Survivors of the treatment were followed up for a median of 5 years. The serial post event echocardiography after the event which include left ventricular function valve function are included. Follow-up data was used to plot survival curves. Results: Of total 66 patients 59 were of stuck mitral valve and 7 stuck aortic valve. The event happened at a mean of $48.86 \hat{\mathrm{A}} \pm 48.80$ month after index operation of valve replacement using mechanical valve prosthesis. The median age is $40.27+10.8$ years with 39 males and 27 females. Thrombolysis was successful in 61 patients with a mortality of 5 (7.57\%). During a mean follow-up of $22.7+20.9$ months 42 patients are alive with $14(22.95 \%)$ patient dead with 5 patients were lost to follow-up. The average follow-up is $18.7+22.7$ months before death. However the survivors have normal LV function and valve function. Conclusion: Prosthetic mechanical valve thrombosis is effectively treated with thrombolysis during acute event with good result. However, during follow-up there is attrition of about $23 \%$ patients within 2 years.

\section{SURGICAL STABILIZATION OF RIB FRACTURES}

Dr Siva Prasad Goud, Dr Balasubramoniam Yashoda Hospital

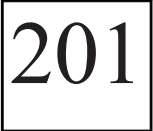

Aims and Objectives: Rib fractures accounted for up to $10 \%$ of all hospitalised cases of polytrauma. Most rib fractures are undisplaced and are managed conservatively. Surgical indications for open reduction and internal fixation (ORIF) of rib fractures are very specific and uncommon. Aim of our study was to analyse the indications for ORIF of rib fractures and their clinical outcomes.

Material \& Method: We conducted a retrospective study on our cohort of 3 patients who had multiple displaced rib fractures and was managed surgically with ORIF. Their postoperative clinical outcome with regards to pain score, hours spent on ventilator after procedure, pain score and duration of hospitalisation was analysed.

Discussion :All 3 patients were having rib fractures \&gt; 4 ribs with hemopneumothorax , 2 patients had flail segment . M: F ratio 2: 1, Age range $34-72$ years. Aetiology Road traffic accident in patient 1 \&amp; 2 , fall from height in patient 3.2 patients needed ventilatory support with inability to wean off being the indication for surgical intervention whereas the $3 \mathrm{rd}$ patient had severely displaced fractures with chest wall deformity. All3 patients received ORIF for 4 ribs ( 4th to 8th rib ) using Titanium bone plates and screws ( Orthomax, India $2.5 \mathrm{~mm}$ ). Preoperative ventilation was 10 days , 7 days and 0 days , extubated 4, 6 and 4 hours post procedure ,pain score reduced from preoperative 8,8 and 7 to 3,3 and 3 in all 3 patients. Patient 1 and 3 were discharged by post op day 6 and 7 while Patient 2 was reintubated by post op day 3 and expired 4 days later due to rebleed associated with head injury.

Conclusion:

$10 \%$ and $34 \%$ mortality is associated with patients having \&gt; 4 \&amp; \&gt;8 rib fractures respectively. ORIF of displaced rib fractures associated with significant chest wall deformity decrease hospital and ICU stay by facilitating weaning off ventilator, decrease incidence of pneumonia by restoring chest wall mechanics and decreasing pain.

\section{A PROSPECTIVE STUDY TO EVALUATE QUALITY OF LIFE OF PATIENTS UNDERGOING MITRAL VALVE REPAIR AND MECHANICAL MITRAL VALVE REPLACEMENT ALONG WITH

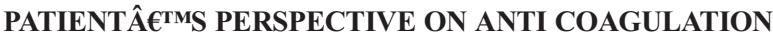 Sai Suraj Kotera, Varghese T Panicker Sree Chitra Tirunal Institute of Medical Sciences \& Technology}

Introduction Declining mortality and major morbidity rates after cardiac surgery have led to increasing focus on patient quality of life (QOL). Beyond longevity, the impact of cardiac surgery on daily functioning is incredibly salient to patients. Objective The primary aim of surgery for mitral valve disease is to improve the overall functional capacity and health of patients. With need for continuous anti coagulation in patients with mechanical prosthetic mitral valve, scope to evaluate for Quality of life is raised. To evaluate Compliance of patients for Anticoagulation and regular follow up.

Methods Prospective Study of 100 consecutive patients who underwent mitral Valve surgery are included. HRQOL will be measured using the Medical Outcomes Study Short Form 12 - health survey (SF-12) and with additional questionnaire related to anti coagulation. Patient will be evaluated before surgery, at hospital discharge, and 6 months post discharge. Results100 consecutive patients who qualified inclusion and exclusion criteria were included. (64males, mean age $52 \hat{\mathrm{A}} \pm 14$ years). 38 underwent Mitral valve repair (MVr) and 62Mitral valve replacement (MVR). Majority patients who underwent MVr had Mitral incompetence. Mean QOL scores (Scale 0-100) for all following surgery showed clinical and statistically significant improvement in Physical function, Role limitation due to Physical function, Social function, General health perception. Patients with EF â\%o¥ 50\%showed higher QOL Scores. Questionnaire regarding Anti Coagulation revealed majority of patients in MVR group considered regular Anti coagulation medication and maintaining INR in range was an additional burden.

Conclusion

Study suggests following Mitral Valve surgery there is significant improvement in QOL of patients who underwent MVr. However patients with impaired LV function pre op were unlikely to demonstrate a significant improvement in QOL in short term follow up. Anti-Coagulation was considered as burden by patients undergoing MVR.

\section{THORACOTOMY APPROACH WITH CENTRAL CANNULATION FOR VSD REPAIR WITH AORTIC VALVE REPLACEMENT: CASE REPORT}

Dr Sunil Dixit, Dr Anil Sharma, Dr. Mohit Sharma,

Dr Sourabh Mittal, Dr Pramod

SMS Medical College, Jaipur

\section{Introduction-}

At present thoracotomy with femoro-femoral bypass is established approach for minimally invasive open heart surgeries butthoracotomy with conventional cannulation is yet to be established. Here we present a case of a 23 year old young female underwent surgical correction of VSD with severe aortic regurgitation via thoracotomy approach with conventional central cannulation. There was no procedure related complication. Repair of VSD with replacement of aortic valve viaantero-lateral Thoracotomy is not documented yet, so to the best of our knowledge its the first reported case of this kind.

Surgical technique- The procedure was performed via Sub-mammary antero-lateral chest incision of about 3 inch. The third intercostal space was opened. Lungs were retracted laterally, Pericardiotomy was done $2 \mathrm{~cm}$ anterior \&amp; parallel toright phrenic nerve to expose aorta, SVC \&amp; IVC. SVC and IVC were looped, Heparinization, all purse-string sutures taken. VSD closure was done with Dacron Patch and 4-0 ethibond pledgeted interrupted sutures and aortic valve was replaced with Cardiamed mechanical valve (size 23). Cannulation, surgery, decannulation and closure as usual. Smooth cannulation and staifactory intracardiac exposure was achieved in all patients. Crossclamp time was 56 minutes, the durationof cardio-pulmonary by-pass was 110 minutes, post-op extubation was done after $12 \mathrm{~h}$, total drain was $350 \mathrm{ml}$ Take home massage- conventionalaorto-caval cannulation from antero-lateral thoracotomy approach for minimal invasive open heart surgery is also a good procedure to minimize the side effects of sternotomy. This procedure is safe to provide the same quality of treatment through a less traumatic and better cosmetic incision resulting in less hospital stay and definitely a lower overall cost. 
PERI-OPERATIVE MORBIDITY AND MORTALITY IN FAILED OFF PUMP CABG PATIENTS - SINGLE CENTRE EXPERIENCE

Faizal Abdul Nishthar, Ravi Kumar T.D., Abdul Rasheed

Government Medical College, Thiruvananthapuram

AIM/OBJECTIVES: Concerns about CPB spurred the development of $\mathrm{OP} C \mathrm{CAB}$. OP $\mathrm{CAB}$ can reduce transfusion requirements, reduce mortality, reduce the risk of stroke, renal dysfunction, and atrial fibrillation. However, the outcomes of patients for whom an off-pump strategy must be changed to an on-pump procedure during surgery have not been extensively studied. From this study we present a single center experience of Peri-operative morbidity and mortality in failed OP CAB patients. Materials and Methods: Retrospective analyses of all CABG cases that happened between January 2016 to November 2019 were analysed. Results: Among them, 550 patients begun and completed on-pump bypass (CPB); 244 patients begun off-pump, of which 229 (93.9\%) completed off-pump coronary artery bypass (OPCAB), whereas $15(6.1 \%)$ were converted to on-pump (CONVERT). CONVERT patients were more severely ill. The observed mortality of CONVERT, CPB, and $\mathrm{OPCAB}$ was $9.9 \%, 3.0 \%$, and $1.6 \%$, respectively, and the observed-topredicted ratio was $2.77,1.20$, and 0.74 , respectively. CONVERT also had more morbidity .

Conclusions Patients who are intended for an off-pump strategy and then require conversion to on-pump have significantly higher operative mortality and morbidity than either completed $\mathrm{OPCAB}$ or $\mathrm{CPB}$ patients. In addition, the operative mortality and morbidity are far in excess of that predicted preoperatively. Based on these results, strong consideration should be given for a planned strategy of CPB for those patients with preoperative hemodynamic instability requiring a salvage $\mathrm{CABG}$ operation, left ventricular hypertrophy, or previous CABG.

\section{CARDIAC AND NON-CARDIAC SURGERY SIMULTANEOUSLY. THE WAY FORWARD FOR CASE EFFECTIVE AND} EFFICIENT WAY.

Pratik Pandey, Lalit Kapoor, Sanjay Dubey, Dhiraj Barman

Rabindranath Tagore international institute of cardiac sciences

Aim/Objective(s)- In a patient who is suffering from a cardiac disease amenable for surgical treatment and also needing a noncardiac surgery for other disease, decision making on which entity should be treated first, is a difficult part. Fortunately, with improvement in surgical techniques, it is possible to conduct combined cardiac and noncardiac operation. here we report two cases of simultaneously operating cardiac and noncardiac part for optimal outcome. Material and method- Double valve replacement was performed simultaneously with laproscopic cholecysctectomy in one case and other case was Coronary artery bypass grafting with mesh hernioplasty was performed for obstructed right inguinal hernia. Result(s) In both cases intraoperative and post operative period was uneventful. There was longer operative time, more blood loss then usual and prolonged length of stay but patient needed single operation theater visit. Conclusion. Simultanreous surgery is feasible and safr for patients suffering from cardiac and noncardiac disease. The short term curative effect is quite satisfactory, and long term survival is expected to be better.

\section{TRANSTHORACIC VERSUS TRANSHIATAL ESOPHAGECTOMY FOR ESOPHAGEAL CARCINOMA: EXPERIENCE FROM A SINGLE TERTIARY CARE INSTITUTION Dr Nadeem Ul Nazeer}

Department of CTVS, SKIMS Institute, Srinagar, J and K, 190011
Background

Thereis a lot of controversy about the best surgical treatment for esophageal carcinoma.

Methods

Inthis retrospective study, 382 patients with carcinoma of the mid-todistalesophagus underwent transthoracic or transhiatal esophagectomy. Early morbidity and mortality were compared. Principal endpoints were disease-free survival (DFS) and overall survival (OS).

Results

Atotal of 177 patients underwent transthoracic esophagectomy, and 205 patients underwent transhiatal esophagectomy. Demographic characteristics and characteristics of the tumor were similar in the two groups. Perioperative and postoperative morbidity was higher after transhiatal esophagectomy. In-hospitalmortality was also higher after transhiatal esophagectomy. The median follow-up was 4.3 years. Estimated 3-year disease free survival (DFS) rates were 44.63 and $31.21 \%$, whereas the 3year overall survival (OS) rates were 57.06 and $41.46 \%$ for the transthoracic and transhiatal groups, respectively (statistically significant). Also, the estimated 5-year DFS rates were 26.55 and $21.46 \%$, whereas the 5year OS rates were 32.76 and $30.24 \%$ for the transthoracic and transhiatal groups, respectively (statistically not significant).

Conclusions

Transhiatal esophagectomy was associated with higherperioperative and postoperative morbidity and in-hospital mortality thantransthoracic esophagectomy. The DFS and OS were higher in the transthoracic group and were statistically significant at 3 years but statistically insignificant at 5 years.

\section{AORTIC VALVE REPLACEMENT}

VIA RIGHT ANTERIOR THORACOTOMY- A 3 YEAR SINGLE CENTRE EXPERIENCE

Andrew Selvaraj, Giridhar Goli, Umar Imran Hamid,

Miles Curtis, Amir Sepehripour, Kulvinder Lall.

Barts Heart Centre, St Bartholomewâ€ $\mathrm{TM}_{\mathrm{S}}$ Hospital, London, UK

\section{Objectives:}

AVR via minimally invasive approaches, is increasingly being advocated for better cosmesis and less post-operative complications. The objective of this study was to report our experience of AVR via Right Anterior Thoracotomy (RAT)

Methods:

We retrospectively analysed records from July 2016 to September 2019. All patients had pre-operative planning CT to assess femoral vessels and ascending aorta. Cardiopulmonary bypass was established via peripheral cannulation of femoral vessels. Sutureless aorticvalves were used.

Results:

26patients underwent AVR via RAT during this period. Mean age was $75+/-6.38 \%$ were males. $30 \%$ were Diabetics. Mean BMI was $29+/-6.93 \%$ had good LV function. Mean Euro SCORE II was $2.2+/-2$. Mean clamp and bypass times were $46 \mathrm{mins}+/-12$ and 79 mins +/- 21 respectively. Two patients were converted to full sternotomy due to bleeding from right ventricle and unsuitable anatomy respectively. Two patients developed wound infections of the peripheral cannulation site. Therewere no reoperations for bleeding and no infections in the thoracotomy wound. Mean post-operative stay was 9 days. Two patients had post-operative stroke which improved at 6 weeks. 3patients had paravalvular leaks ( 2 mild, 1 moderate). There were no in hospital mortalities.

\section{Conclusions:}

Aorticvalve replacement via RAT can be carried out with low mortality and morbidity,albeit with an increased stroke risk as seen in this case series. A higher incidence of embolic stroke with peripheral cannulation has been reported, advocating the use of central arterial cannulation for risk reduction. 
MANAGEMENT PROTOCOL FOR

RUPTURED SINUS OF VALSALVA

ANEURYSM : AN 8 YEAR EXPERIENCE

Dr. Yogesh T, Prof. Durga Prasad Reddy, Dr. Mayuri,

Dr. Vikas S R, Dr. Subhash, Dr. Sathyanarayana, Dr. Veena

Vydehi Institute of Medical Sciences \& Research Centre

\section{BACKGROUND:}

Ruptured sinus of Valsalva aneurysm (RSVA) isrelatively uncommon. Variety of surgical strategies are available for the surgical treatment of ruptured sinus of Valsalva aneurysm (RSVA) from simple primary closure to patch closure.

OBJECTIVE: We reviewed our 8-year experience and current literature regarding the efficacy of different surgical approaches.

METHODS: A retrospective review identified 18 patients who underwent RSVA repair between 2006 and 2014. The mean age was 25 $\hat{\mathrm{A}} \pm 5$ years. The RSVA originated from the right coronary sinus in 14 patients $(78 \%)$, from the non-coronary sinus in $4(22 \%)$.Dual-chamber exposure (the involved chamber and aorta) was used in $67 \%$ of the patients and isolated trans-aortic approach was used in $33 \%$ patients. RSVA was repaired with either a patch $(n=14)$ or direct sutures $(n=4)$, aortic valve was replaced in eight patients among the study group.

RESULTS: There was no mortality. The follow-up was available in 16 patients $(88 \%)$ of survivors ranging from 1 month to 5 years.

CONCLUSIONS: Surgical treatment for RSVA carries a very low operative risk and long-term freedom from death and reoperation. The therapeutic approach must be chosen according to the ruptured chamber and associated lesions. Patch repair of RSVA carried much lower morbidity and hence must be preferred over primary closure.

\section{OUTCOMES OF TOTAL PERICARDIECTOMY FOR CONSTRICTIVE PERICARDITIS- A SINGLE CENTRE EXPERIENCE OF 19 YEARS}

Dr. Utkarsh Sanjay Sanghavi, Dr. Anagha Tulsi A, 209 Aditya Doddamane, Dr. Chittaranjan S. J,

Dr. Hiremath CS, Dr. Shekhar Rao

Sri Sathya Sai Institute of Higher Medical Sciences, Bengaluru

\section{Background:}

Constrictive pericarditis (CP) is the result of a spectrum of primary cardiac and non-cardiac conditions. It represents a form of severe diastolic heart failure (HF), secondary to a noncompliant pericardium. This study is designed to evaluate immediate and short term outcomes of total pericardiectomy clinically and echocardiographically.

Objective: To analyze the short-term and mid-term outcomes of total pericardiectomy using death and adverse events as end points.

Methods: 83 patients with constrictive pericarditis underwent total pericardiectomy between January 2001 and August 2019 in the Department of Cardio-Thoracic \&amp; Vascular Surgery, Sri Sathya Sai Institute of Higher Medical Sciences, Whitefield, Bengaluru. Results: The mean age was $30.4 \hat{\mathrm{A}} \pm 13.35$ years. The mean post-operative CVP was $8.73 \hat{\mathrm{A}} \pm 3.06 \mathrm{~cm}$ of $\mathrm{H} 20$. Histopathological results showed tuberculouspericarditis $43(51.82 \%)$ in patients, chronic constrictive pericarditis in patients, purulent pericarditis in 1(1.20\%) patient and viralpericarditis in $1(1.20 \%)$ patient. At the end of 12 months, $75(92.60 \%)$ patients were in NYHA class I and 6(7.40\%)patients were in NYHA class II.

Conclusion: Tuberculouspericarditis is still the most frequent cause of $C P$ in developing country like India. Total pericardiectomy via median sternotomy with or without the use of CPB is the surgical treatment of choice and should be performed as early as possible as it is associated with lower mortality, less postoperative low-output syndrome, early normalization of hemodynamics and better long-term survival.
LVAD IMPLANT IN THE ELDERLY

Varun Bansal, Vivek Rao

Toronto General Hospital, Toronto, Ontario, Canada

Aim: To analyze the results of left ventricular assist device (LVAD) implant in patients over the age of 65 with end-stage heart failure Methods: We performed a single center, retrospective review of 198 consecutive patients who underwent continuous flow LVAD implant between 2006 and 2019. Patients were divided into three groups based on the age at the time of surgery. Group A consisted of patients below 60 years of age $(\mathrm{n}=$ 125). Group B consisted of patients between 60 and 65 years of age $(n=$ 39). Group $C$ consisted of patients above 65 years of age $(n=34)$. We compared 30 day mortality between groups. In addition, we performed a comparative analysis of complications related to anticoagulation (stroke, gastro-intestinal bleeding, and thromboembolism), wound (infection, bleeding, and reoperation), organ dysfunction (liver or renal dysfunction) and device-malfunction. Results: Group $\mathrm{C}$ patients showed significantly higher 30-day mortality $(\mathrm{n}=11,32 \%)$ compared to Group A patients $(\mathrm{n}=$ $10,8 \%)$ or Group B patients $(n=7,18 \%)(p \& 1 t ; 0.001)$. However, the cumulative incidence of early complications were comparable between the three groups ( $\mathrm{p} \& \mathrm{gt}$; 0.05).Conclusion: LVAD implant in elderly patients is associated with higher 30 day mortality, but comparable morbidity to patients under the age of 60 . However, compared to the natural history of end-stage heart failure in the elderly, LVAD therapy should still be considered in highly selected recipients.

\section{ROLE OF EMERGENCY CABG IN THE ERA OF PERCUTANEOUS INTERVENTIONS}

Dr Chandana N C, Prof Venugopal Rama Rao

Sri Jayadeva Institute of Cardiovascular Sciences and Research

Background : In acute coronary syndrome, prompt restoration of myocardial blood flow is essential to optimize myocardial salvage and decrease mortality. Coronary artery reperfusion, if performed in a timely manner improves clinical outcomes compared to no reperfusion. Percutaneous interventions and fibrinolysis can restore blood flow in an acutely occluded coronary artery in most of the patients; but in a few subset of patients coronary artery bypass graft is needed to effectively restore blood flow in acutely occluded vessels. Methods : We conducted a study between March 2016 â€" February 2018 analysing pre-operative, intra-operative and post-operative data of patients undergoing emergency CABG. Patients who underwent emergency CABG were those with an acute myocardial infarction who had an ongoing ischemia and were unfavourable for percutaneous interventions, or those who had failed percutaneous interventions or those who developed complications during PCI. Patients who were excluded from the study were those with mechanical complications following MI and stable patients after 7 days of MI. Results : 57 patients underwent emergency CABG during the study period with males constituting $94.7 \%$ of the population. $78.9 \%$ of them had an ejection fraction less than $35 \%$. Half of them presented with low cardiac output status requiring inotropic support and IABP and 15\% of them required mechanical ventilation. In our data, a total of 51 patients $(89 \%)$ underwent beating heart surgery and only $6(10 \%)$ of them required conversion to on pump surgery. These patients received an average of 3.77 grafts, ensuring that all segments with flow limiting lesions with adequate target size received revascularisation. $15 \%$ of these patients had increased post operative bleeding and $10 \%$ were re-explored. Patients were extubated 2-24 hrs after surgery and IABP was removed 30-36 hours later. They were discharged 7 days later from the hospital. 3 patients died due to multi-organ failure due to intractable low cardiac output. Conclusion : Immediate surgical revascularisation of patients presenting with acute MI is feasible and leads to excellent results. Emergency CABG not only treats the culprit lesion but achieves a complete revascularization and offers a clear advantage for the patient. This might be true especially 
for patients with multi-vessel disease and/or LMCA stenosis. Surgical patients are at increased risk of re-exploration when operated within the first three days. The optimal timing of CABG for patients with acute MIremains difficult to establish. Management should involve cardiothoracic surgeons and interventional cardiologists as part of a Heart Team approach. Keywords : Emergency CABG, ACS, Urgent CABG, Failed PCI

\section{OUR EXPERIENCE OF 19 YEARS IN THE MANAGEMENT OF PARTIAL ANOMALOUS PULMONARY VENOUS CONNECTIONS}

Dr. Dinesh kumar. R, Dr. Anagha Tulsi A., Aditya Doddamane, Dr. Naveen. P, Dr. Hiremath.C.S, Dr. Shekhar Rao.

Sri Sathya Sai Institute of higher medical sciences

Background: Partial anomalous pulmonary venous connection withatrial septal defect is a condition which needs to be corrected surgically. Over years various surgical techniques have been evolved with each has its ownadvantage and disadvantage.

Objective: To analyze the clinical outcomes of patients undergoing single patch repair, double patch repair and Wardens procedure.

Methods: Four hundred and fifty five patients with PAPVC underwent surgical correction in our institution between June 2001 and April 2019 in the Department of Cardio-Thoracic \&amp; Vascular Surgery, Sri Sathya Sai Institute of Higher Medical Sciences, Whitefield, Bengaluru.

Results: There was no in hospital mortality in our study. In the immediate post-operative period, one patient from single patch group (0.21\%)developed atrial flutter and another patient from two patch repair group developed atrial bigeminy $(0.21 \%)$ both were managed medically. The atrial flutter was reverted to normal sinus rhythm at the time of discharge whereas atrial bigeminy remained upto 6 months follow-up which was managed medically. None of the patients had sinus node dysfunction requiring pacemaker insertion. Two patients in Wardens group developed an anastomotic gradient of $6 \mathrm{mmHg}$ and $13 \mathrm{mmHg}$ respectively at 3 months follow-up but they were asymptomatic.

Conclusion: The single patch, 2 patch or caval division techniques provides excellent surgical outcomes for PAPVC repair or PAPVC associated with atrial septal defect. There was no incidence of significant SVC obstruction, pulmonary venous obstruction or sinus node dysfunction in our study. There were 2 patients with rhythm disturbances one each in the single and double patch groups. Though the twopatients who underwent Wardens technique had SVC gradient, they wereasymptomatic. It could be attributed to right atrial appendage trabeculations. We recommend sizing the patch adequately for intra-atrial baffle, careful placement of sutures well away from the pulmonary vein orifice to prevent pulmonary venous obstruction and thorough excision of right atrial appendagetrabeculations and a wide anastomosis to prevent SVC obstruction. We recommend regular follow-up for detection of the mentioned complications.

\section{MICS FOR DUMMIES}

Alok Mathur, Akshay Sharma

CK Birla Hospitals/RBH, Jaipur

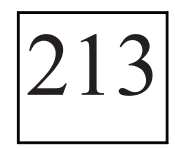

Aim: Handhold the uninitiated through basic concepts and pitfalls of MICS surgery. Method: With the experience of over 500 MICS procedures, a video-graphic excerpt of live cases is prepared. The presentation targets the pitfalls in each step of the procedure starting from femoral access to venous drainage, perfusion pressure, myocardial protection, de-airing and exposure. The potential complications and methods to avoid or tackle the are also discussed. Results: The video guide aims at standardizing the MICS procedure and avert disasters during the early experience of new surgeons. Conclusion: An attempt to standardize MICS training.

\section{PRIMARY SOLITARY FIBROUS} TUMOR OF THE MEDIASTINUM: A RARE CASE REPORT

Navneet Kumar Srivastva, Tripti Verma,

Manoj Kumar, SS Rajput, DK Srivastava, LM Joshi, AP Jain

Dr Ram Manohar Lohia Institue of Medical Sciences, Lucknow, Uttar Pradesh

Introduction: Solitary fibrous tumour ( SFT) of the pleura is a rare primary tumor arising from mesenchymal cells subjacent to the mesothelium. We report a case of primary mediastinal SFT. A 65 year old male presented with retrosternal chest pain and breathlessness for last six months. ECG and echocardiography showed no significant abnormality. Chest X-ray and CT scan revealed a large mediastinal mass. Left anterolateral thoracotomy was done for the complete resection of the mass. Grossly it was a well circumscribed partly encapsulated and lobulated mass measuring $14 \mathrm{~cm} \mathrm{X} 10 \mathrm{~cm}$ X $8 \mathrm{~cm}$. On cut section it was firm, solid and white. Histopathological examination revealed a spindle cell tumor disposed in a pattern less architecture. The tumor showed hypocellular and hypercellular areas separated by thick hyalinised collagen and staghorn vessels. No nuclear atypia, mitosis or necrosis was evident. On immunohistochemistry tumor cells were positive for CD34, STAT6 and Bcl 2 while negative for vimentin ,SMA,S-100 and desmin. Discussion: A wide range of neoplasm both primary and metastatic occur in the mediastinum which emphasize considerable diagnostic difficulties. One of the closest differential of SFT is monophasic synovial sarcoma which is a malignant tumour with a poor prognosis. Enbloc resection of the mass is curative in SFT however chemotherapy is a must in Synovial sarcoma. Conclusion: Although very few cases have been reported in literature it is important to identify this tumour. It has a good prognosis and only $8 \%$ local recurrence amenable to curative re-excision.

RETAINED ARTERIAL CATHETER IN RADIAL ARTERY- EXPLORATION AND REMOVAL : A RARE COMPLICATION

Dr R Punithakumar M.S., Mch., DNB, Dr Suresh Babu Kale

M.S., Mch., DNB., MNAMS, Dr Chennakesavulu M.D., D.M Meenakshi Hospital, Thanjavur, Tamilnadu

Arterial cannulation is a very useful way of establishing continuous monitoring of blood pressure. In addition, arterialcannulation allows reliable access to the arterial circulation for the measurement of arterial oxygenation and for frequent blood sampling. Ischemic damage, sepsis, local infection, pseudoaneurysm, hematoma, and bleeding are the complications that can be rarely encountered in patients with arterial line. Complications related to arterial cannulation occur in fewer than $1 \%$ of patients. Spontaneous fracture and retention of a part of the arterial catheter within the arterial system is very rare. We report a case of spontaneous fracture of arterial catheter and retention of a part of the catheter within the radial artery which was confirmed with ultrasound. Surgical exploration of the radial artery revealed a long segment arterial catheter which was removed successfully

\section{INDIA'S FIRST ACUTE TYPE A AORTIC DISSECTION REPAIR USING A THORAFLEX HYBRID PROSTHESIS}

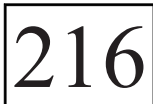

Dr. A. Mohammed Idhrees, Dr. K. Mukesh, Dr. Ketak Nagare, Dr. Mohamed Ibrahim, Dr. Aju Jacob, Dr. Bashi V Velayudhan SRM Institutes for Medical Science (SIMS Hospital), Chennai, India 
Introduction Acuteaortic dissectionis a catastrophic condition, for which emergency surgery is the mainstay of therapy. In approximately $70 \%$ of patients who survive surgery, a dissected distal aorta remains, posing a risk of late aneurysmal degeneration, rupture, and malperfusion, and secondary extensive interventions are often required. The Frozen Elephant trunk technique is a promising approach in patients with acute dissection. Case History A 66 years old male presented with back pain of 1 day duration. His CT aortogram showed Type A aortic Dissection. HIs ECHO showed mild aortic regurgitation. He underwent emergency surgery. Frozen Elephant trunk procedure was performed using Thoraflex hybrid prosthesis- replacing his ascending aorta, arch and proximal descending thoracic aorta. The postoperative course was uneventful. The patient was discharged home on postoperative day 7.Three months later, the patient continues to do well. Conclusion Frozen elephant trunk is a viable option in acute Type A aortic dissection taking care of the tears in the aortic arch and proximal descending thoracic aorta. Also it promotes thrombosis of false lumen.

\section{SOME SIMPLE INNOVATIVE TECHNIQUES TO MAKE CARDIO-VASCULAR \&THORACIC SURGERY SIMPLER AND SAFER \\ Harinder Singh BEDI, Jatin Singh, Vikram Arora Ivy Hospital, Mohali, Punjab}

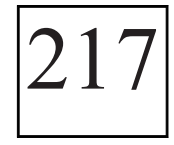

OBJECTIVE: The KISS approach (Keep It Safe \&amp; Simple) works well in life and it has been shown to work well even in high tech situations. Over the years we have developed some simple ways to make cardiac surgery simpler, more efficient and cost effective, clutter free and safer in the OR. METHODS:

A Backward technique to achieve forward progress: Use of retrograde perfusion to relieve acute ischemia eg left main or critical CAD or cath lab mishap with ECG changes or unstable hemodynamics. Use of an electric drill in severely calcified aortic Indigenous emergency atraumatic aortic cross Use of a dental syringe as a mist blower (even a root canal can have its advantages) Use of a pulse oxymeter trace to optimise IABP timing Using an oxygen blower to stay cool in a beating heart surgery where the ambient temperature is kept normal to avoid cooling the patient. 7. Use of a nasal speculum to put in a chest tube with the least possible trauma to the lung: Use of a nasal speculum for better visualisation in a case of a sub-aortic membrane9. Use of an endo light to look for an additional muscular Use of spigots to fix the ends of tourniquets â€" this avoids using clamps and so reduces clutter. Use of a snare on valve sutures after bringing the valve into the annulus this acts like a surrogate tie. 12. Avoidingan extra aortic root sump (vent) line by adding a $Y$ to the venous return cannula A safe method for ligating a PDA The Bedi-IMA Buttress to avoid a BPF The Bedi cooling turban in carotid surgery Use of 3D printing to help during surgery

RESULTS: The above techniques have been used for the last 15 years with safety and comfort.

CONCLUSIONS: We advocate simplifying routine matters with the aim of improving safety and efficiency inthe OR.

\section{PERIOPERATIVE TROP-T LEVELS AND OUTCOMES POST CORONARY ARTERY BYPASS SURGERY}

Dr. Yousuf Mohammed Rafi, Dr. Satish Govindaiah, Dr. Vivek Siddaiah, Dr. Manish Pangi

Sri Jayadeva Institute of Cardiovascular Sciences and Research

\section{INTRODUCTION:}

Coronary Artery Bypass Grafting (CABG) can be associated with significant perioperative and postoperative myocardial damage and necrosis, which may occur in varying degrees. Intraoperative injury may result from cardiac manipulation, inadequate myocardial protection, and intraoperative defibrillation; in addition, postoperative myocardial injury may be associated with acute loss of bypass grafts. Cardiomyocyte necrosis may be detected through measurement of cardiac enzymes such as Troponin-T, CK, CKMB

AIM

1) To assess if Preoperative Trop-T levels predict Risks and outcomes of CABG in patients With Recent Myocardial Infarction2) To assess if elevated post-operative Trop-T levels co-relate with post-operative Myocardial infarction3) Determination of the threshold of Trop-T levels that are associated with an adverse postoperative outcomes, including myocardial infarction after CABG4) To determine if non-infarct elevation of Trop-T can occur secondary to increased myocardial handling, hematomas, intra-myocardial coronaries MATERIALS AND METHODS:

This was a prospective, single centre, single surgeon, observational study. A total of 100 consecutive patients who underwent CABG during a 1-year period at Sri Jayadeva Institute of Cardiovascular Sciences were enrolled. Comprehensive clinical details were collected for each patient.

Variables recorded were

Preoperative

$\hat{A} \cdot$ Clinical History, Examination, Demographics, past medical history, prior medication use, cardiac catheterization results, preoperative ECG ECHO,

Cardiac enzymes, Â.Trop-T levels were performed for all patients on the morning of surgery $\hat{A} \cdot 2 \mathrm{D}$ ECHO, and ECG were repeated 1 day prior tosurgery to exclude new-onset MI during stay In hospital Intraoperative

$\hat{A} \cdot$ Type of surgery, Number of bypass graftsconduits used, Quality of vessels, infarction, RWMA, intraoperative complications

Postoperative

Â.ECG: on shifting patient to recovery and Postoperative Day 1

$\hat{A} \cdot$ Cardiac enzymes: CK, CKMB, Trop-T within 12urs of procedure $\hat{A} \cdot$ Length of stay in ICU

$\hat{A} \cdot$ Post operative ECHO: performed on Post-operative Day 2

OutcomeMeasures

$\hat{A} \cdot$ New onset $\mathrm{ST} / \mathrm{T}$ changes, $\mathrm{Q}$ waves/LBBB

$\hat{A} \cdot$ Need for IABP use

$\hat{A} \cdot$ Need for vasopressor agents post-operatively

$\hat{A} \cdot$ New RWMA on post operative ECHO

$\hat{A} \cdot$ Postoperative heart failure

$\hat{A} \cdot$ All cause Mortality by 30 days.

RESULTS

A total of 100 consecutive patients undergoing CABG were selected. $(\mathrm{N}=100) .48$ patients had elevated preoperative Trop- $\mathrm{T}$ levels (48\%), with a mean Trop-T level of 0.247 (Range:0.024-3.28)

Preoperative Echocardiography showed RWMA in 72 patients(72\%). Positive troponin was associated with moresignificant comorbid conditions and more extensive coronary artery disease.

There was no increase in mortality inpatients with elevated preoperative Trop-T. $(p=0.07)$. Finding of intra-myocardial coronary, hematomas corelated with increased post-operative Trop- $\mathrm{T}(\mathrm{p}=0.03)$

Elevated post-operative Trop-T (as definedas 5 times normal reference range) did not co-relate with post-operative myocardial infarction $(\mathrm{p}=0.092)$, as per $2 \mathrm{DECHO}, \mathrm{ECG}$, Clinical assessment

Conclusions

Pre-operative Trop- $\mathrm{T}$ is a poor independent predictor of outcomes following CABG. Post-operative Trop-T levels do not co-relate well with postoperative myocardial infarction. Larger studies are needed to definite threshold level of post-operative Trop-T for predicting myocardial infarction, in conjunction with clinical data.

A UNIQUE CASE OF CRHD, POST DVR WITH STUCK AORTIC PROSTHETIC VALVE\& PREMATURE CAD WITH MULTIPLE CO MORBIDITIES. REDO STERNOTOMY- CABG, AVR \& TV REPAIR DONE.

Zubair Rashid

KIMS secunderabad 
36 years lady,a k/c/o CRHD since 2004 presented with AOE III for few days, intermittent not associated with SOB, Palpitations. She was hospitalized and evaluated and found to have premature CAD (LMCA ) and two vessel coronary artery disease. She had underwent Double valve replacement (Mitral \&amp; Aortic) in 2009 and PTMC in 2004.Her 2DE revealed Stuck Aortic prosthetic valve, severe TR,mild PAH, normal Mitral Prosthesis. She had history of Appendicitis, Polyarthralgia and cervical spondylitis and was on treatment. She was taken up for surgery under high risk group. CABG with LAD and $\mathrm{Om}$ anastomosis with sphaneous vein graft with Aortic valve replacement with SJ Mechanical valve and tricuspid repair with 3D tricuspid ring. No major intraop or post op issues .Patient has retained her sinus rthym. She had an uneventful post operative stay. Was discharged in a stable condition.

\section{A RARE CASE OF MEDIASTINAL HYDATIDOSIS WITH SEVERE AORTIC STENOSIS}

Anuj Sangal, Ratnamalika Kumar, Ashok Gupta,

Rajneesh Malhotra

Max Superspecialty Hospital

Objective-Surgery for Mediastinal Hydatidosis with Severe Aortic Stenosis-A rare case report. Method and Results-We report a case of a Severe Aortic Stenosis with mediastinal location of hydatidosis in a 75year-old Arab man admitted for chest pain. The chest radiograph showed a rounded and homogeneous opacity. Computed tomography showed a mid anterior mediastinal cyst, with two small liver sites. Through a midline sternotomy, we found a large cystic mass in the anterior mediastinum. The patient received a cystectomy followed by Aortic valve replacement with medical treatment based on albendazole. He improved a few weeks later. Conclusion-Mediastinal cysts remain rare, even in endemic countries, which makes initial diagnosis difficult. The diagnosis is based on typical clinical and radiological criteria. Our observation shows the importance of keeping this diagnosis in mind when a patient presents with signs of mediastinal compression.

\section{SINGLE CONTINUOUS SUTURE TECHNIQUE IN MITRAL VALVE REPLACEMENT \\ Prof. James Thomas, Dr. Abhay Jain, MS, M.Ch \\ D.Y. Patil University Hospital, Administration}

3rd Floor, Sector 5, Nerul, Navi Mumbai 400706.

Interrupted pledgetted or non pledgetted sutures have been in practice for Mitral Valve Replacement for many years. Single Continuous sutures techniques have been adopted by some for some time. From 2007 to 2019 we have evaluated single continuous suture technique in 435 Mitral Valve Replacements. Periodic Echo evaluation and chest Xrays have been done. Age group varied from 7 years to 62 years and Females were $62 \%$. Pre operative Echo cardiographic evaluation was done in all patients including chest Xrays. Mitral Stenosis (varying from 0.6 to $1 \mathrm{~cm}$ ), Mitral Regurgitation moderate to severe, mixed liesions, calcification of the Valve varying from moderate to severe with Annulular calcification or other wise. Left aterial enlargement varying from 4.5 to 6.6 was the average. Single 2-0 polypropylene suture was used for the continuous technique. Valve excision was done preserving posterior Mitral Leaflet most of the time. Sutures begins at 90 'clock position Valve to annulus is taken and then the Valve is lowered and the suturing continued in the anti clock wise position making sure sutures are taken close without cutting through. Even moderate calcification of the annulos was not a deterrent. Bileaflet Valves were used and the positioning was 2 O'clock to 7 O'clock Post operative evaluation of the Valve function including gradient and paravalvular leaks were done for 10 years follow up. Post operative Mitral Regurgitation of trivial to mild were seen in $4.5 \%$ patients who were mostly asymptomatic. The details are presented. The technique ensures safe, faster, reliable replacement of the Valve.
OFF PUMP CORONARY ARTERY BYPASS

GRAFTING FOR MULTIPLE VESSEL ATHEROSCLEROTIC CORONARY ARTERY DISEASE IN A YOUNG BOY

FAMILIAL HYPERCHOLESTEROLEMIA.

Dr.Sana Firdouse, Dr. Alla Gopala Krishna Gokhale, Dr. Bharadwaja, Dr. Subhramanyam, Dr. Ravi Shankar Apollo Hospitals, Jubilee hills

Aim/objective: To Present a case of OPCAB in 14 yr young boy with familial hypercholesterolemia. Material and methods: A 14 year old boy presented to dermatology opd with extensive xanthomas over the body. One valuation, his mean plasma cholesterol was 618 , LDL was 549 and HDL was 23, suggestive of Familial Hyperlipoprotenemia (Fredrickson classification- type 2b). His father had sudden cardiac death in 3rddecade of life. His homocystein was high(54.45) . In view of strong family history and high lipid levels, he underwent CAG which showed Left main disease with triple vessel atherosclerotic disease. OPCABG x 3 grafts LIMA RIMA Y, LIMA TO LAD, RIMA to Ramus and SVG to PDA was done using octopus myocardial stabilizer. After surgery patient was started on dietery modifications and high dose statins. Data was further collected from hospital records, discharge summary regular follow up of records. Result: Patient underwent OP CABGx 3 grafts on 31/7/ 2019. Hispost operative period was uneventful. Discharged on 6th postoperative day. Conclusion: The patients with familial hyperlipoprotenimia have very high chances of developing severe coronary and extra coronary atherosclerotic lesions. Studies have shown that $\mathrm{CABG}$ is associated with decrease in mortality rates in these patients. Use of arterial grafts, high dose statins and a holistic approach to patient management improves the overall survival in them. Management of primary predisposing factor is essential if identified like in this case for good long term results. If $\mathrm{CAD}$ is identified during preop evaluation of liver transplant in patients with familial hyperlipoprotenimia, OPCAB technique may have an advantage over on pump technique in combined surgeries, in reducing postop morbidity and mortality. However long term close follow up with regular pharmacotherapy is necessary.

\section{CONSERVATIVE MANAGEMENT OF HEMODYNAMICALLY STABLE BLUNT TRAUMA THORAX PATIENT WITH DRAIN OUTPUT 1.5 LITRE AFTER INSERTION OF CHEST DRAIN}

DR Vikram Halder,DR Pankaj Aggarwal. Assistant Professor

Department of CTVS, PGIMER Chandigarh

Postgraduate Institute of Medical Education and

Research

Author - Dr Vikram Halder .Senior Resident Department of CTVS PGIMER ChandigarhPhone-8420033905 . Email -

vikramavnrt@gmail.comCo Author - Dr Pankaj Aggarwal . Assistant Professor Department of CTVS PGIMER Chandigarh

A twenty two year old male patient with blunt trauma abdomen and blunt trauma thorax due to physical assault was admitted with chest pain and respiratory distress. After primary survey on thoracic examination right sided breath sound was diminished and on percussion dullness was found in right side and abdomen was soft non tender .Patient was hemodynamically stable and rt sided chest drain was inserted. Chest drain was inserted around two hour after the incident. After insertion of chest drain around one and half litre blood came out. Chest drain was clamped and routine investigation was sent and blood product was given. On chest $\mathrm{x}$ ray and CECT thorax and abdomen moderate right sided collection with pneumothorax were found. Declamping of drain was done intermittently and output was gradually decreased and there was no drop in serum hemoglobin. On serial CXR lung was expanded and drain output was decreased to less than $25 \mathrm{ml}$ per day. Drain was omitted on 7 th day .On 
CXR after drain removal minimal collection was present .patient was discharged.

\section{MANAGEMENT OF TRAUMATIC BRACHIAL ARTERY INJURIES : AN EXPERIENCE AT A HIGH VOLUME TERTIARY CARE CENTER \\ Rahul Bose, Goutam Sengupta \\ IPGMER and SSKM Hospital, Kolkata}

\section{BACKGROUND ANDOBJECTIVE:}

The most frequently injured artery in the upper extremity is the brachial artery. The purpose of this study was to evaluate our experience in diagnosis and management of these injuries and associated injuries and their subsequent clinical outcomes.

\section{PATIENTS ANDMETHODS:}

Twenty seven patients underwent surgery at our institute from August 2018 to September 2019. Pre operative diagnosis was done by physical examination or Doppler ultrasonography. Patients were taken to the operating room at varying intervals of time following their arrival in the ER. This was mostly dictated by their mode of presentation.

RESULTS:

This study group consisted of 25 males and 1 female, ranging in age from 4 to 67 years with a mean age of 29 years. The mechanism of injury was penetrating in 25 cases and blunt in 2 cases. Cut injury to the limb with as hard of glass was the most common form of penetrating trauma. Treatment included primary arterial repair in 7 cases, end-to-end anastomosis in 7 cases, interposition vein graft in 13 cases . Other associated injuries apartfrom trauma to the artery included venous injury (8), bone fracture (2), and peripheral nerve injury (4). Ten patients developed postoperative complications. One patient underwent an above-elbow amputation.

CONCLUSIONS:

Early diagnosis and treatment of traumatic brachial artery injury can lead to successful limb salvage and minimize morbidity and loss of function associated with an above elbow amputation. Debridement of non viable tissues and identification of concomitant injuries is vital to improve outcomes.

\section{RSOV IN A CASE OF VSD AR} COMPLICATED BY INFECTIVE ENDOCARDITIS Dr.Kumar.P, Prof. Kumsi Sreedhar, Dr. Madhuprakash Sri Jayadeva Institute Of Cardiovascular Sciences

And Research, Mysore

Patient was taken for emergency surgery and was found to have BAV, perforated anterior leaflet, ruptured RSOV into RVOT through a VSD, vegetations. AVR and RSOV closure was done after debridement

\section{OFF PUMP CABG IN A PATIENT WITH CHRONIC MYELOID LEUKEMIA \\ Dr. N. Madhusankar, Dr. A. Aarimuthuswamy, Dr. R. Anantharaman, Dr. K. M. Cherian \\ Frontier Lifeline Hospital}

Aim and objective :

In patients with haematological malignancy, use of cardiopulmonary bypass (CPB) for coronary artery bypass surgery is a cause for concern. This report describes a case of off pump $\mathrm{CABG}$ in patient with chronic myeloid leukemia (CML)

Material and method:

A 60 year old male who is diabetic and normotensive, presented with complaints of chest pain for one day and he was diagnosed as a case of
ACS .Non ST elevation myocardial infarction. He gives past history of low grade fever, joint pain for which he was evaluated and diagnosed as a case of CML 8years back; he is on regular treatment (with oral Nilotinib) under hematologist care with. His 2 Dimensional echocardiography revealed Moderate LV dysfunction, Ejection fraction- 44\%, RWMA+, mild Mitral regurgitation. His coronary angiogram which revealed double vessel disease of LAD/ RCA. His preoperative Haemoglobin level 10.8 gm\%, total count8000 cell/cumm, Platelets 2.66 lakhs/cumm and his pre-operative coagulation profile was within normal limits. He underwent off pump coronary artery bypass surgery with grafts to Left internal mammary artery to LAD, SVG to Diagonal and distal RCA. He had uneventful intraoperative and post operative period. He was discharged 7 th post operative day.

Conclusion:

Surgical strategy in patients with hematological malignancy must be planned and carried out with specific aim of decreasing post operative complications. Cardiopulmonary bypass affects all system including hematopoietic system. Off pump coronary artery bypass surgery is extremely safe option in these patients.

\section{NEONATAL THORACOABDOMINAL THROMBOTIC AORTIC OCCLUSION IN A CASE OF TRANSPOSITION OF GREAT ARTERIES: A RARE CASE REPORT}

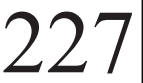

DR NIKUNJ, B R Jagannath, Dr. R Shastri, Dr. Kishore Jayanthi, Dr Sairam Palaparthi, Dr. Ramesh Babu Dasari

Star Hospitals, Banjara hills, Hyderabad

Introduction: The arterial switch operation (ASO) is the treatment of choice for transposition of the great arteries. Thoraco abdominal aortic occlusion in association with transposition of great arteries has not been reported in literature. Background: 21day-old full-term male child was diagnosed with Dtransposition of great arteries (dTGA) with intact ventricular septum and was admitted for elective ASO. Pre- operatively baby had worsened respiratory distress with hypotension and required mechanical ventilation. Bilateral femoral pulses were feeble with dampened pressure trace in femoral arterial line and normal trace in the axillary arterial line. No Coarctation was found on repeat echocardiography. Case report: Patient underwent emergency uneventful ASO. A small thrombus was retrieved from the pulmonary artery during the procedure. Post procedure, there was significant gradient between femoral and axillary pressures but needle pressure of proximal-mid DTA revealed normal pressure trace. Patient was shifted to intensive care unit in hemodynamically stable state with moderate inotropic support. However, patient developed low cardiac output and haemodynamic instability over next 12 hours with cardiac arrest on Post-operative day (POD) -1. Patient was resuscitated, was reexplored for surgical bleeding and hemostasis was secured. Conventional angiography was undertaken in view of persistent femoroaxillary gradient which revealed a large clot in thoraco-abdominal aorta till bifurcation with no visualization of renal and mesenteric arteries and with unsuccessful thrombo-suction. Thrombolysis was tried as a last resort; however, the distal vessels could not be opened up. Thoraco-abdominal endarterectomy was considered but in view of completely occluded renal and mesenteric vessels of long duration with poor distal run-off, it was not attempted. Low cardiac output with anuria persisted and patient expired on POD1.Conclusion: Neonatal arch thrombosis was described previously. Neonatal thoraco-abdominal thrombotic aortic occlusion is an unreported and unusual complication associated with dTGA. The possible causes and its management options are open for discussion. Keywords: Transposition of Great Arteries, Arterial Switch Operation, Aortic Occlusion

PRIMARY CARDIAC LYMPHOMA A RARE PRESENTATION OF LEFT ATRIAL MASS Vijay Shewale, Chaitanya Raut Lokmanya Tilak Municipal Medical College 
Background- Primary cardiac lymphoma ( PCL ) is defined as extranodal lymphoma involving only the heart and /or pericardium. The most common histological type of PCL is diffuse B cell lymphoma. Here, we reported rare case of PCL. Case Report- 20 years old female presented with acute pulmonary edema in april 2018. ECHO showed large mass noted in left atrium obstructing mitral valve with minimal blood flow across mitral valve, severe tricuspid regurgitation, severe pulmonary hypertension. Patient was immediately taken for left atrial mass excision. Findings large lobulated hard mass occupying whole of LA with dense adhesions. Histopathology and Immunohistochemistry suggestive of high grade ALK positive anaplastic large cell lymphoma

\section{PROPHYLACTIC PREOPERATIVE LEVOSIMENDEN VERSUS INTRA -AORTIC BALLOON PUMP FOR OFF-PUMP CORONARY ARTERY BYPASS GRAFTING SURGERY IN PATIENTS WITH LEFT VENTRICULAR EJECTION FRACTION $<25 \%$ : SINGLE CENTERED RETROSPECTIVE STUDY}

Dr Ritesh Mate, Dr Chaitanya Raut, Dr Prashant Mishra,

Dr Jayant khandekar

Lokmanya Tilak Municipal General Hospital and Medical College Mumbai, Maharashtra

Aim and Objective: To study hemodynamic efficiency and immediate outcome of prophylactic infusion of levosimendan versus preventive IABP in patients with the LV dysfunction undergoing OPCAB surgery. Materials \&amp; methods: 60 patients undergoing elective OPCAB with LVEF (\&lt;25\%) were randomly divided into Two groups ( $\mathrm{n}=30$ each), namely, levosimendan (Group L), IABP (Group B) group. In group L Patients received a preoperative dose of levosimendan initial bolus of 12 $\mathrm{mg} / \mathrm{kg}$ for $10 \mathrm{~min} 24 \mathrm{hrs}$ before surgery, followed by $0.1 \mathrm{mg} / \mathrm{kg} / \mathrm{min}$ for $24 \mathrm{~h}$. In Group B Patients IABP was inserted $24 \mathrm{hrs}$ before surgery continued for at least $24 \mathrm{hrs}$. Hemodynamic monitoring included heart rate, invasive blood pressure, cardiac index $(\mathrm{CI})$, pulmonary capillary wedge pressure (PCWP), Vasoactive inotropic score (VIS) and arterial blood gases with serum lactates at various time intervals during and after surgery. Results: CI was greater and PCWP reduced significantly in both the groups during intraoperative and early postoperative period. Serum lactate concentration was lower in Group B as compared to group L. Incidence of postoperative atrial fibrillation (POAF), low cardiac output syndrome (LCOS), and acute kidney injury was less in both the group. Intensive care unit stay was significantly shorter in the levosimendan group as compared to IABP group. Conclusion: Preoperative levosimendan is as effective as IABP in terms of maintaining stable hemodynamics in njIntraoperative and early post operative period in patients with the LV dysfunction (LVEF \& lt; 25\%) undergoing OPCAB surgery and reduces the incidence of LCOS, POAF and conversion to CPB.

\section{MULTIPLE PRESENTATIONS OF INTRA-CARDIAC TUMORS OUR EXPERIENCE OVER LAST 4.5 YEARS}

Dr. Gaur Hari Das

R.G. Kar Medical College and Hopsital, kolkata

Background

Cardiac tumours are rare with prevalence at autopsy ranges from $0.001 \%$ to $0.3 \%$. These tumours with multiple symptoms, accurate diagnosis is imperative for appropriate treatment and prognosis. We report clinical features, surgical management and post-operative complications of cardiac tumor patients in our institute.

Aims and objectives

1. To study modes of presentation and clinical spectrum in different age groups
2. To know outcome of different approaches in surgery

3. To know complications associated

Material and Method

A retrospective review of 3-year period revealed 7 cases of cardiac tumour in patients with mean age of 41.4 years (range 35-60 years) and male: female of 4:3, presented to the CTVS department of R. G. Kar Medical College, Kolkata. Analysis was by presentation, location, interventions, histological diagnosis.

Result

Mean age of presentation was 41.4yrs with male predominance (1.3:1). Most common symptom Dyspnoeain 3 patients (42.8\%), palpitation in patients $(28.5 \%)$, fever in 1 patient $(14.2 \%)$ and 1 patient $(14.2 \%)$ was asymptomatic. Histologically all tumors were benign, most common Myxoma in 5 patients (71.4\%), Lipomatoushypertrophy in 1 patient (14.2\%), haemangioma in 1 patient (14.2\%).No pre-op tissue diagnosis was obtained. Most common location- Left atrium (57.1\%).Approach through LA in 4 patients $(57.1 \%)$, through RA in patients (28.5\%), Biatrial approach in 1 patient (14.2\%). Intra-op, 1 patient had ventricular tachycardia managed by DC shock. In our series, commonest complication was arrhythmia in 2 patients(28.5\%). Postoperative period was uneventful. Intensive care unit stay ranged from 3 to 5 days (median 3.6 days). In-hospital mortality was $0 \%$,and 30 -day survival was $100 \%$. There were no deaths and no recurrence during 18 months of follow-up. Conclusion

Primary cardiac tumors are clinically rewarding cases with straight forward diagnosis and less morbidity and mortality post surgery. Incidence is low, mostly diagnosed by Echocardiography. High index of suspicion is required for diagnosis. Earlydiagnosis \&amp; treatment is key to low mortality and morbidity.

RIGHT ATRIAL THROMBUS AFTER MINIMALLY INVASIVE ATRIAL SEPTAL DEFECT CLOSURE: A CASE REPORT

Dr. Vinita Sharma, Dr. Rakesh Chittora, Dr. Mukta Bhatnagar, Dr. Rohit Singh

Fortis Escorts Hospital, Jaipur

Right atrial thrombus formation is a rare complication of surgical closure of ASD. We are reporting a case of RA thrombus formation following minimaly invasive ASD closure in a 15 years old male patient on two year follow up. We managed the case with oral anticoagulants to maintain INR of 2.5 and Low molecular weight heparin (LMWH) injections. The thrombus resolved in 5 days of starting treatment without evidence of pulmonary embolism. Key Words: ASD, Thrombus, Anticoagulants, LMWH, Pulmonary embolism.

\section{EFFECT OF POSITIONING OF HEART DURING OPCAB ON STATIC AND DYNAMIC COMPLIANCE OF LUNG.}

Shrikant Suryawanshi, Prashant Mishra, Shakuntala Basantwani, Jayant Khandekar, Chaitanya Raut. Lokmanya Tilak Municipal Medical College and Sion General Hospital, Mumbai.

Heart displacement during off-pump coronary artery bypass grafting (CABG) is necessary to expose the coronary arteries. This may lead to increase in right, left atrial pressure, pulmonary congestion and reduction in forward flow. This will be reflected by changes in the compliance of lung as it indirectly reflects pulmonary artery pressure. We analysed the changes in dynamic and static compliance of lung in relation to the grafted arteries. Aims and objectives-The primary aim was to monitor changes in dynamic and static compliance of lung respectively during different positions of heart. The secondary aim was to correlate the changes in lung compliance with the changes in the cardiac output 
Materials and Methods-

In this prospective study the relationship between surgical exposure and lung compliance (Static and Dynamic) was assessed in 60 consecutive patients undergoing OPCABG utilizing the Octopus Tissue Stabilization System. Cardiac index was assessed using Vigileo (Edwards) monitor.

Results-

Cardiac index decreased significantly after target stabilization during all coronary anastomosis. The greatest decrease was noted during OM anastomosis. Ionotropic drugs were required to maintain mean arterial pressure \&gt;60 $\mathrm{mm} \mathrm{hg}$ in 43 patients which was most frequently noted during $\mathrm{OM}$ anastomosis.

Similarly, fall in static compliance was noted in all coronary anastomosis, however, the maximum fall of $4.5 \%$ was noted during PDA anastomosis (but statistically not significant) p- 0.478 . Similarly $5.5 \%$ fall in dynamic compliance was noted during PDA anastomosis.

\section{CONCLUSION-}

During OPCABG using octopus for coronary target stabilization, the fall in static and dynamic compliance was observed which decreased the most during PDA anastomosis. The fall in cardiac output was most during OManastomosis. Careful monitoring of respiratory and haemodynamic variables are of utmost importance to avoid conversion to ON PUMP surgery.

\section{SYSTEMATIC REVIEW OF CONSECUTIVE CASES OF DECORTICATION OF PLURA FOR VARIOUS ETIOLOGY IN LAST 2 YEARS AND ITS OUTCOME}

Dr Rakesh Kumar, Dr Hemant Narayan Roy

Rajendra Institute of Medical Sciences (RIMS), Ranchi

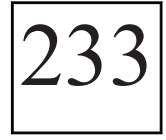

Aim-Our aim to focus the variety of etiology of lung collapse and postsurgixal outcome of these cases. Material and methods-We selected consecutive last 2 years 25 cases in our study. These patients underwent decortication of plura for variety of reasons in last 2 years in our hospital RIMS Ranchi. They were $60 \%$ male and $40 \%$ female. Age group from 7 years to 65 years. Common etiology were tubercular(40\%), infective(30\%), traumatic(20\%) and neoplastic(10\%). Results-We noticed excellent result in cases of traumatic lung collapse. Poor result in tubercular cases. Variable result in neoplastic cases. Most of the cases underwent successful lung expansion post surgery with relapse of disease in 2 cases out of 2 cases in follow up upto 2 years. Conclusion- 1 . Timely intervention and prior optimization (spirometry, infection control, antitubercular drug, intercostal drainaige) before surgery is mandatory for better outcome.2. Adequate decotication with minimal air leak and full lung expansion is key of success. Disclaimer- no conflict of interest to diclose.

\section{STUDY TO ASSESS KNOWLEDGE OF ANTICOAGULATION AGENTS AND ANTICOAGULATION CONTROL IN PATIENTS POST MECHANICAL VALVE PROSTHESIS SURGERY AT A TERTIARY CARE HOSPITAL.}

Dr Chaitanya Raut, Dr Noaman Shaikh

Lokmanya Tilak Municipal General Hospital, Sion, Mumbai.

Aim: To assess knowledge of anticoagulation agents andanticoagulation control in patients on anticoagulation therapy post cardiac valve replacement surgeries with metallic valve prosthesis at a tertiary care hospital using an institutional validated questionnaire. Methods: A questionnaire was framed and was subjected to face validity, content validity and pilot testing. The reliability of the questionnaire was evaluated by examining its internal consistency (Cronbachs a); test-retest reliability (1month) was assessed with the intraclass correlation coefficient and our value of 0.82 was considered significant. The patients post valve replacement surgery on regular follow-ups in OPD, for INR monitoring at least 6months post surgery with at least 3 follow-ups were considered for the study.

These patients were subjected to answering of the questionnaire. Scores were calculated out of 20.Results: A total of 690 patients in the period of 1year met the inclusion criteria, of which 15 patients refused consent and hence were rejected. 28 people of the 675 participants, scored a perfect 20/20 score. 434 patients had a score ranging from16-19. 184 patients had a score of 11-15. 23 patients scored between 6-10. 6patients had a score of less than 6. Of the subsections of the questionnaire, the patients scored most poorly in the â $€ œ F o o d$ and Drug interactions, where only 35 people scored 3/3.

Conclusion: There is a need for increasing patient in formation and awareness regarding anticoagulation drugs and their importance and interactions to enhance patient safety.

\section{EXTRA-ANATOMICAL ASCENDING} TO DESCENDING THORACIC AORTA BYPASS FOR TREATMENT OF AORTIC COARCTATION IN ADULTS: OUR EXPERIENCE

Arnab De, Kuntal Surana, Noaman Shaikh, Tejas

Dandekar, J V Khandekar, Prashant Mishra,

Chaitanya Raut, Vaibhav Shah, Manish Jadhav

Lokmanya Tilak Municipal Medical College and Hospital

\section{Introduction:}

Coarctation of aorta $(\mathrm{CoA})$ isa relatively rare diagnosis in adults. Several operative approaches are available for the surgical correction of CoA. Young adults usually present with hypertension refractory to pharmacotherapy, exercise intolerance, headaches andangina.

Aim:

To analyse the operative outcomes of patients undergoing extraanatomical bypass at our institution.

Methods:

A retrospective analysis of 8 patients operated for $\mathrm{CoA}$ in our institution from 2013 to 2018 was done. All patients were diagnosed preoperatively with a CT Aortogram. All baseline investigations including a transthoracic echocardiography were done for all patients. All patients underwent median sternotomy and were put on cardiopulmonary bypass after cooling to $32 \hat{\mathrm{A}}^{\circ} \mathrm{C}$. PTFE graft of appropriate size was sutured to descending thoracic aorta after incising the posterior part of thepericardium. The other end was anastomosed to the ascending aorta with the graft placed along the right ventricle. Results were analysed in terms of duration of hospital stay, need for re-operation, reduction of blood pressure immediately post procedure, and recurrence of symptoms or CoA. Patients were followed up at 3rd, 6th \&amp; 12th months.

Results:

The mean age of the patients was $32 \hat{\mathrm{A}} \pm 12$ years. 5 patients were male and 3 were female. No in-hospital deaths occurred. All patients had significant reduction in blood pressure in the immediate post operative period and the effect was maintained throughout the follow-up period. 2 patients needed re-exploration due to bleeding.

Conclusion:

Extra-anatomical bypass is a safe approach for adult CoA treatment with less surgical risk and good outcome.

\section{COMPOSITE LIMA-GSV GRAFTING STRATEGY- DOES IT STAND THE TEST OF TIME?}

Jeevesh John Thomas, Bhaskar Ranganathan, Simon Philipose, Shylesh Kumar, Jose Chacko Periapuram Lisie Heart Institute, Lisie Hospital, Kaloor, Kochi

\section{AIMS and OBJECTIVES}

Composite arterial grafts using a configuration from the left internal mammary artery (LIMA) are commonly used for coronary artery surgery. 
Data is limited regarding the use of Saphenous vein (SV) in composite grafts from the LIMA. This is a very useful â€œaorta- no touchâ€ technique for complete revascularization in patients with hostile aortas. This study aims to determine whether LIMA or vein patency was reduced by making a composite graft using LIMA and vein.

\section{METHODS}

Data was collected from all patients who underwent a composite LIMAGSV grafting during CABG. There were 25 patients who underwent this technique in the period 2012 to 2019. Their operative notes were analyzedand their immediate and short term follow ups were obtained from case files. They were further contacted over telephone or direct hospital visit to inquire about their functional class. A TMT was obtained in all possible cases. A coronaryangiogram was also obtained from those who had an indication.

RESULTS

The total number of CABGs performed during this period was 6800 . Composite grafts were done using the radial or Right Internal Mammary Artery (RIMA) as a Y graft in about $40 \%$ of CABGs. GSV was anastomosed to the LIMA in cases of hostile aorta or very short segment of usable conduit that prevented a direct graft from the aorta. Follow up was not obtained for 5 patients as they were lost to follow up or untraceable. There were 3 deaths-one in the perioperative period, 1 after 6 months and 1 after 2 years. They were all unrelated to the technique of grafting. 10 of the remaining 17 patients were in NYHA I. There were 5 patients with severe left ventriculardys function preoperatively which made them to be in NYHA II or III. A few angiograms are pending as of now.

CONCLUSION

A LIMA- GSV y graft strategy is a valuable skill for a surgeon when facing a diseased aorta or shortage of good conduit length. A detailed study of how this graft works in the long term is yet to be known, however, short and intermediate results are encouraging.

\section{A CASE OF SPORADIC RIGHT ATRIAL MYXOMA PRESENTING AS ACUTE PULMONARY THROMBO-EMBOLISM DR Kannan G, DR Rajan M, DR RM Krishnan, DR S Kumar, DR K Balamurugan Meenakshi Mission Hospital and Research Centre}

INTRODUCTION Myxomas are the most common primary cardiac tumors. Right Atrial Myxomas account for $10 \%$ of cardiac myxomas. Most Atrial Myxomas arise from Inter Atrial septum. About $10 \%$ arise from posterior wall, anterior wall or appendage. Myxomas usually present with constitutional symptoms. Pulmonary embolism occurs in less than $10 \%$ of right atrial myxomas and may cause fatal pulmonary obstruction. CASE REPORT A 32 years old male presented to our hospital with acute onset breathlessness with S1Q3T3 pattern on ECG and acute right pulmonary artery thrombus on CT Pulmonary Angiogram. He was thrombolysed elsewhere and referred to our centre. Trans esophageal echocardiogram showed mobile mass in right atrial free wall. Patient was taken up for surgery under moderate hypothermia and total cardiopulmonary bypass. A pedunculated mass of size $2 \mathrm{X} 1 \mathrm{~cm}$ in anterolateral RA free wall was excised. Patient was weaned of bypass after RA closure. Patient was started on oral anticoagulants post operatively. DISCUSSION We experienced a case of 32 year old male with acute pulmonary embolism attributed to embolization of degenerating tumor fragments from sporadic RA myxoma. Pulmonary thrombo-embolism is reported to occur in approximately $3.2 \%$ of myxoma patients. Pulmonary embolism is the most dreaded and devastating complication of right sided myxomas. CONCLUSION In patients presenting with sudden onset of dyspnoea especially young patients, tumor embolization from right sided heart tumors should be kept in differential diagnosis. Timely echocardiogram can help in identifying right atrial myxoma as the potential cause for pulmonary embolism.
A CASE REPORT OF CHYLOTHORAX WITH CYSTIC HYGROMA OF NECK (GRAHAM STOUT DISEASE)

Kollapalli Goutham, Amaresh Rao Mallempatti

Nizams Institute of Medical Sciences

A26 yr old lady came to our OPD with complaints of dyspnea and cough associated with sputum for which she was treated outside and a chest tube was inserted. The patient initially had cough with expectoration white in colour, loss of weight and recurrent episodes of fever .she was evaluated and found to have T. B and had taken A.T.T for 6months - 1 yr back . Later she found to have increasing dyspnoea with recurrence of cough and again was evaluated with chest $\mathrm{x}$ - ray, which showed collection present in the right side of chest. Fluid was aspirated and sent for analysis The patient had a recent onset neck swelling in right posterior aspect of neck with fluctuation and transillumination positivity The patient on evaluation had recent loss of tooth spontaneously and now has been reported to have osteolyticlesions in the right 1 st and 3rd ribs and D12 vertebrae. Gorham-Stout disease (GSD) is a rare bone disorder characterized by progressive bone loss (osteolysis) and the overgrowth (proliferation) of lymphatic vessels. GSD is thought to be caused by an error in the development of the lymphatic system. In individuals with GSD, bones become infiltrated with lymphatic vessels and are broken down and replaced by a fibrous band of connective tissue.

\section{IMPACT OF LV DYSFUNCTION ON SHORT TERM OUTCOMES AFTER OFF PUMP CORONARY ARTERY BYPASS Ajay Kumar Thangaraj, Sanjay Theodore} Chettinad Hospital and Research Institute

Objective: Pre-operative left ventricular function is an important determining factor of post-operative morbidity and mortality in patients undergoing coronary artery bypass procedure. The purpose of our study is to compare early outcomes in patients with good, mild, moderate and severe LV function after off pump coronary artery bypass.

Materials and method: We included 950 patients with $79 \%$ of male and $21 \%$ of female, who underwent OPCAB during the period August 2014 toOctober 2019. Patients were divided into four groups. Group I comprised 525patients with EF\&gt; $45 \%$, group II had 242patients with EF$41 \%-45 \%$, group III had 134 patients with EF-36\%-40\%,group IV had 49 patients with EF\&1t;35\%. All groups were compared on the basis of preop variables like demographic features, intra-operative variables like number of grafts, complete revascularisation and post-op variables like ventilation hours, ionotropic supports, re-exploration, transfusion requirement, hospital stay and mortality.

Result: There were no demographic differences between groups. Intra-operatively, moderate and severe LV patients received greater number of grafts $(p=0.002)$ and insertion of $\operatorname{IABP}(p=0.008)$ than other groups. Post operatively, the hours of ventilator support and post-operative stay (mean - 4days) were longer in severe LV patients. There was no significant intergroup difference in other variables

Conclusion: OPCAB inpatients with poor LV function can be performed safely with the same quality in terms of complete revascularisation and encouraging discharge rates as inpatients with normal LV function.

NEED OF THE HOUR' STRUCTURAL HEART INTERVENTION TRAINING FOR TRAINEE SURGEONS IN INDIA Â€“" A MAGIC PORTION TO REVITALIZE OUR SPECIALITY Dr. Srikanth K, Professor P.S. Jairaj

Department of Cardiothoracic Surgery, Narayana

Hrudayalaya, Bangalore 
Introduction

The Heart team concept has been in existence for quite sometime now but the role of surgeons in such 'teams in interventions is grossly dwarfed by the technical prowess of our physician counterparts who can produce commendable results with the puncture of a needle. The newer intervention techniques are here to stay and grow. As surgeons, we need to train ourselves to perform these procedures, if not, it would make us mere spectators as the number of interventions snowball. The future cardiothoracic surgeon has to don the role of an 'interventional surgeon' who has to be as much familiar with the needle as with his scalpel.

Aim To propose and propagate the idea of introducing training modules for Indian cardiothoracic surgical trainees in learning catheter-based intervention techniques

Materials and methods

We reviewed published literature to understand the importance laid on wire skills in cardiothoracic surgical training programmes in India and abroad, assessed the involvement of surgeons/ trainee surgeons in the cath lab to perform interventions. The information on various specialised training programmes available for surgeons to learn catheter skills after their super-speciality training were analysed. We also discuss measures taken at our institute to expose our residents to the world of interventions.

Results

Most of the foreign publications showed an active involvement of the surgical team including residents in performing interventions both as the primary operator and as a companion to the cardiologist with one American study quoting that almost77.5\% of the surgeons having performed TAVI as the primary operator or in close conjunction with the cardiologist, which is in stark contrast to the situation in our country where almost all the interventions are performed by cardiologists whilst surgeons are approached only in the event of complications.

Conclusion

With better understanding of cardiovascular anatomy, superior tissue handling techniques and the ability to tackle complications, surgeons are equally, if not better equipped to handle interventions with ease. With several hybrid procedures coming up, this is the best time for surgeons to embark on the intervention journey. This should not be misconstrued as an attempt to disrupt the physician-surgeon harmony but only as an enhancement of the mutual partnership to provide the best possible results. At a time when there is a debate on the sustenance of our speciality, innovative training modules for the budding surgeons will go a long way in ensuring a smooth transition from the knife-wielding surgeon to the wire-handling surgeon.

\section{CORONARY ARTERY BYPASS GRAFT IN A KIDNEY TRANSPLANT PATIENT, LESSONS TOI BE LEARNT \\ Manpreet Singh Salooja, Ajeet Bana, Ashish Madkaiker, Sandeep Tirkey, Tania, Bishal Gautam \\ Eternal Hospitals, Jaipur, Rajasthan}

AIMS and objectives: Indications for cardiovascular surgery, postoperative complications, and outcome were recorded in a renal transplant recipient. Cardiovascular disease is the major cause of mortality in patients with advanced chronic renal failure and in those undergoing renal transplantation. Several factors, such as hypertension, alterations in lipid metabolism, diabetes, and immunosuppressive therapy, accelerate cardiovascular disease in renal transplant patients and in those undergoing dialysis. It is worth noting that myocardial infarction as a cause of death in these patients exceeds $20 \%$. The first myocardial revascularization surgeries in renal transplant patients were performed in the beginning of the 1970s 7-9. Since then, not only have transplantation and immunosuppression techniques greatly developed, but dialytic methods have also. This has resulted in an expressive and progressive increase in these patients' survival, making this population subject to coronary atherosclerotic disease part of the daily routine of cardiovascular surgery services. Material And
METHODS A 59 YR male with history of renal transplant in 2008 was admitted a hospital in Jaipur with history of chest pain and dyspnoea. After diagnosing ischemic coronary artery disease, he was reffered to our unit. Patient underwent a succesful operation of triple vessel coronary artery disease by bypass grafting on beating heart. RESULTS Patient was discharged on postoperative day 7th without any complication. BUN and Creatinine levels at discharge were $20 \mathrm{mg} / \mathrm{dl}$ and $1.20 \mathrm{mg} / \mathrm{dl}$ CONCLUSION In conclusion, myocardial revascularization in renal transplant patients may be performed with no definitive renal lesion or loss of the transplanted kidney.

\section{SURGERY FOR TYPE A AORTIC DISSECTION - A SINGLE CENTRE EXPERIENCE}

Patralekha Das, Pradeep Narayan, Biswarup Purkayastha, Atanu Saha Rabindranath Tagore International Institute of Cardiac Sciences

Aim/Objectives-Surgical management of Type A aortic dissection is a challenging area however, advancement in surgical, perfusion and neuroprotective measures has resulted in significant improvement in outcomes. Aim of this study was to assess the contemporary institutional outcomes in a tertiary care centre. Materials and method-66 consecutive patients undergoing repair for aortic dissection were included in the study. Data was recorded about the demographics, operative and post-operative outcomes prospectively in our database and was analyzed in a retrospective fashion. Axillary artery cannulation, open distal anastomosis and antegrade cerebral perfusion was standard. Results-Majority of patients $[\mathrm{n}=49(75 \%)]$ underwent aortic root replacement (Bentallâ $€^{\mathrm{TM}_{\mathrm{S}}}$ Procedure). Along with hemiarch[3(4.5\%)], total arch $[6(9 \%)]$ and interposition grafts $\hat{\mathrm{A}} \pm$ aortic valve repair/replacement $[6(9 \%)]$. A hybrid procedure was required in $6(9 \%)$ patients. Mean CPB time was $352+121$ minutes with aortic cross clamp time of $282+77$ minutes. Mean antegrade cerebral perfusion time was $148+28$ minutes. Despite extensive surgery, adverse neurological outcomes were seen in only $3(4.5 \%)$ of patients. Post-operative renal failure was seen in $4(6 \%)$, gastro-intestinal complications in $1(1.5 \%) .30$ day mortality was seen in $6(9 \%)$ patients. Conclusion-Our results, overall outcome in general and neurological outcomes in particular, compare favourably with published literature. Adoption of open distal anastomosis, axillary cannulation, antegrade cerebral perfusion and other neuro-protective measures can provide improved outcomes in this challenging area of cardiac surgery. Hybrid procedures allow management of more complicated cases and prevention of future complications.

\section{THE CARDIAC SURGEON'S EXISTENTIAL CRISIS - A ROOT CAUSE ANALYSIS \\ Dr Srikant Mohapatra \\ Healthworld Hospitals, Durgapur (W.B)}

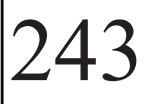

The scope of cardiac surgery has narrowed down significantly over the past decade or so, hamstrung by adverse government policies \&amp; regulations, rising costs of quality healthcare, socio-medico-legal issues, and last but not the least, the proactive interventional cardiologists' forays into uncharted territory. We delve deep into data available on public platforms \&amp; sample surveys to present a root cause analysis of the problem in the Indian context and suggest remedial measures for course correction. Address for communications: Dr Srikant Mohapatra Flat no. 10G, Kanakangan Residency City Centre, Durgapur West Bengal. PIN 713216e-mail: drsrikantm@gmail.com Contact no: 9937245670

\section{IMPLICATIONS OF THE SAVR VS TAVR CONTEST : IS IT ABOUT SAFETY \& LONGEVITY OR POPULARITY \& PROFITABILITY? \\ Dr Srikant Mohapatra}

Healthworld Hospitals, Durgapur (W.B) 
TAVI is the flavour of the season, with multiple cardiolosist's fora proclaiming it as the best bet for aortic stenosis in high, moderate even selected low risk surgical candidates. We analyze the data presented in the landmark trials comparing TAVI \&amp; SAVR and try to read between the lines of actual data to assess the gap between evidence \&amp; practice. We also look into the ethical angle of the process of approval of medical devices in the context of TAVI. Address for communications: Dr Srikant Mohapatra Flat no. 10G, Kanakangan Residency City Centre, Durgapur West Bengal. PIN - 713216e-mail: drsrikantm@gmail.com Contact no: 9937245670

\section{COMMON ATRIUM WITH MIDLINE INFERIOR VENA CAVA AND LEFT SUPERIOR VANA CAVA DRAINING INTO LEFT ATRIUM WITH CLEFT IN ANTERIOR MITRAL LEAFLET-A INTRAOPERATIVE SURPRISE WITH SUCCESSFUL BIVENTRICULAR REPAIR}

Dr Anish Gupta, Dr Namrata Gaur, Dr Anshuman Darbari, Dr Sandeep Gautam AIIMS, Rishikesh

A three year old male child presented with history of cyanosis and recurrent lower respiratory tract infections since birth. He had resting saturation between $80-90 \%$ and diagnosed as common atrium with severe mitral valve regurgitation and cleft in anterior mitral leaflet. During surgery, presence of midline inferior vena cava and left superior vena cava draining into left atrium below left atrial appendage was intraoperative surprise. Although it was little difficult, we were able to reroute LSVC and IVC to right atrium without producing any obstruction to systemic or pulmonary veins and biatrial size was normal. the cleft in AML was closed and child had mild mitral regurgitation after repair. He had mild lower repsiratory tract infection in post operative period otherwise he did well. The take home message is that patients with complex and rare anomalies should get $\mathrm{CT}$ angiography before surgery and there is paucity of literature and videos of corrective surgery to the best of our knowledge.

\section{PEDIATRIC CARDIAC SURGERY IN THE HILLS-A CURVY ROAD \\ Dr Anish Gupta, Dr Anshuman Darbari,}

Dr Namrata Gaur, Dr Raja Lahiri, Dr Deepak Kumar

Satsangi, Dr Sandeep Gautam, Dr Ajay Mishra

AIIMS Rishikesh

AIIMS rishikesh is one of six autonomous apex health care institutes established under Pradhan Mantri Swasthya Suraksha Yojna which started in 2012. The department of CTVS started thoracic and vascular procedure and finally with the arrival of heart lung machine in 2018, open heart surgeries were started. Keeping a slow but steady pace, after performing successful adult cardiac surgeries, we started pediatric cardiac surgery programme this year and we are coming up as world class tertiary health care in government sector in the state of Uttarakahand and neighboring states. we describe the hurdles and obstacles that came in the path of starting a new pediatric cardiac setup especially in difficult hilly terrains and how we overcame them so that we are now able to equalize the imbalance in tertiary health care in remote hilly areas of northern part of our country. We also discuss our future plans and some innovative frugal ideas that helped us to sustain our pediatric cardiac surgery program. we hope our experience will benefit many future surgeons willing to help the needy by starting new setups for pediatric cardiac surgery across the country keeping in view the increasing prevalence of congenital heart disease and paucity of good centres and surgeons doing congenital cardiac surgery which has led to increased waiting lists in already established centres.
CARDIAC ECHINOCOCCOSIS: A RARE ENTITY

Dorendro Thingujam, Dr Birla Roy D Gnanamuthu

Christian Medical College, Vellore

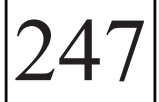

Introduction:

Hydatid cyst is very common in India. Cardiac echinococcosis is an extremely rare condition and pericardial involvement is an even rarer entity.

Background: Hydatid cyst mostly affects the liver, lungs, muscles, bones and less commonly the kidneys, spleen and brain. Cardiaccysts are usually seen in the left ventricle, few are found in right ventricle and interventricular septum. Isolated pericardial cyst is very rare.

Case Report: A 70 year old female patient presented to our department with intermittent non exertional left sided chest pain. 2D-Echo \&amp; Computed Tomography showed a hydatid cyst of size 77 x $76 \times 87 \mathrm{~mm}$ compressing the left ventricle. Midline sternotomy was done, pericardialhydatid cyst was excised completely. Patient was discharged on the 6th post-operative day without any complications with regular follow up for the last 2 years.

Conclusion: Early diagnosis and treatment is important because of fatal complications following cyst rupture. 2D Echo and CT are the gold standard diagnostic methods. Surgery is the best treatment modality.

\section{PSEUDOANEURYSM OF ASCENDING AORTA: A RARE COMPLICATION AT RARE SITE}

Dr Rahul Bhushan, Dr(Prof) Vijay Grover, Dr(Prof)

V K Gupta, Dr(Prof) Narender S. Jhajharia

ABVIMS and RML Hospital, New Delhi

\section{Introduction:}

Pseudoaneurysmof ascending of aorta (PAA) is a rare complication after cardiac surgery with incidence of less than $0.5 \%$. Here we present an unusual case of PAA where site is external curvature of ascending aorta significantly away from previous aortic suture line. We reiterate the importance of focused surgical strategy which prevented catastrophic haemorrhage and permitted satisfactory surgical management.

CASE SUMMARY

24year, female presented at out patient clinic with progressively increasing painless pulsatile swelling of size $4 \mathrm{~cm} \tilde{A}-4 \mathrm{~cm}$ above sternal angle to the right of midline scar since four months. She had underwent mitral and aortic valve replacement one year back for severe aortic and mitral incompetence due to infective. CT angiography of swelling revealed pseudoaneurysmal sac of $6 \mathrm{~cm}-6 \mathrm{~cm}$ size arising from right margin of ascending aorta. CPB and Totalcirculatory arrest (TCA) with antegrade cerebral perfusion instituted throughePTFE graft anastomosed (end to side) to right common carotid artery. Pseudoaneurysmal sac of size $8 \mathrm{~cm}-8 \mathrm{~cm}$, filled with fresh blood, clot and thrombus were evacuated with repair.

DISCUSSION

Pseudoaneurysmof Aorta has been reported at suture sites after valve replacement surgery. But in this case rent was at external curvature of aorta away from suture site. Diagnosis of PAA is itself an indication of surgery, due to its fatal sequalae like rupture, erosion or compression of surrounding structure and bleeding fistula formation. Important consideration of surgical management includes establishment of extra-mediastinal $\mathrm{CPB}$ and hypothermic circulatory arrest prior to opening chest.

AUTOLOGOUS PERICARDIAL MITRAL VALVE RECONSTRUCTION: EARLY EXPERIENCE AND RESULTS.

Kaushik Ramachandran, Prasanna Simha Mohan Rao , Rakesh Naik Lachma, Prabhu Manohar

Sri Jayadeva Institute of Cardiovascular Sciences and Research 
BACKGROUND/AIM: Mitral valve repair is considered as the gold standard treatment for degenerative mitral valve diseases. But these reparative techniques are not always successful in addressing all rheumatic valves effectively. Rheumatic heart disease still contributes to the majority of the mitral valve disease burden in our country. Irreparable heavily calcified rheumatic valves traditionally undergo mitral valve replacement which is associated with the need for anti-coagulation, thrombohemorrhagic complications and progressive LV dysfunction. These complications can be avoided by performing autologous pericardial mitral valve reconstruction in irreparable mitral valves. We share the early results of the same from our centre.

MATERIALS AND METHODS: Inspired by our experience with the Deac procedure of total autologous mitral valve reconstruction in 200809 , which showed good structural integrity of the valves on follow up, we designed a new technique of auto-pericardial mitral valve reconstruction for calcified mitral valves. The procedure has been standardized at our institute. 11 consecutive patients who satisfied our inclusion criteria have successfully underwent the procedure.

RESULTS: Autologous pericardial mitralvalve reconstruction had several favourable outcomes including low gradients,excellent orifice area, lack of anticoagulation etc. None of the patients hadany thrombohaemorrhagic complications. There were no conversions to prosthetic valve reconstruction.

CONCLUSION: Autologous pericardial mitral valve reconstruction is a feasible alternative to prosthetic valve replacement inirreparable heavily calcified mitral valves.

\section{TOTALLY ENDOSCOPIC MITRAL VALVE REPAIR: EARLY EXPERIENCE , RESULTS, PITFALLS AND LESSONS LEARNT}

Prabhu Manohar, Prasanna Simha Mohan Rao, Rakesh Naik Lachma, Jeeva Vijayan,Kaushik Ramachandran Sri Jayadeva Institute of Cardiovascular Sciences and Research

Background/Aim: Mitral valve repair is considered the gold standard for the treatment of many mitral valve pathologies, especially degenerative diseases. Nowadays, it is effectively performed via several minimally invasive approaches all over the world, with excellent peri-operative outcomes. The aim of our study is to share the early outcomes of patients who underwent totally endoscopic mitral valve repair in our institute. Materials and methods: From July 2017 till date, a total of 45 patients underwent mitral valve repair through totally endoscopic approach, via $3 \mathrm{~cm}$ peri-areolar (males) and sub-mammary (females) incision. Several repair techniques were used endoscopically such as ringannuloplasty, neochordae implantation, commisurotomy, posterobasal chordalrelease, AML peeling electrocautery maze etc. We incorporated techniques fromlaparoscopic/general endoscopic surgery like puppeteering stitches etc to make the procedure more effective Results: The mean age was $33 \hat{\mathrm{A}} \pm 13$ years and $40 \%$ of the patients were males. There were 31 isolated mitral valve repairs and rest were combined procedures. There were 3 conversions to lower midline hemi sternotomy due to injury to RV, LV and pulmonary artery in the beginning of our experience. There were two mortalities. At 6 months follow-up, none of the patients had any significant regurgitation. None them underwent any intervention in the follow up period. We describe our learning curve and pitfalls and lessons learnt during the learning curve Conclusion: Totally endoscopic mitral valve repair is a safe and feasible procedure with favorable peri-operative outcomes and good success rates.

\section{PERIOPERATIVE AND LONG TERM OUTCOME OF THYMECTOMY IN MYAESTHENIA GRAVIS - SINGLE CENTRE EXPERIENCE AND PROGNOSTIC ANALYSIS}

Manoop B,T D Ravikumar

Government Medical College, Thiruvanathapuram
Aim - Thymectomy is an established treatment of myaesthenia gravis. This study is aimed at assessing perioperative and long term outcome of thymectomy in myaesthenia gravis patients and to identify prognostic factors after thymectomy. Materials and methods - The clinical data of 62 consecutive thymectomies performed between 2016 - 2019 were retrospectively reviewed for clinical efficacy and variables influencing outcome. Assessment was done by Kaplan - Meier survival curves and cox proportional hazards regression analysis. Results - Mean operative blood loss was $137.87+/-165.25 \mathrm{ml}$. The postoperative crisis increased with preoperative MG and the prescription dose of anti cholinesterase. Complete stable remission was the end point for evaluation of treatment results. Overall five year CSR was $57.5 \%$. Two good prognostic factors were identified, preoperative prescription of anti cholinesterase alone ( $\mathrm{p}=0.035)$ and non thymomatous $\mathrm{MG}(\mathrm{p}=0.003)$. The five year survival rate of ocular type of $\mathrm{MG}$ reached a high level of $67.4 \%$.Conclusion Thymectomy can achieve good long term CSR in MG. High dose prescription of anti cholinesterase and advanced stage by Myaesthenia Gravis foundation of America have higher risks of postoperative crisis. Preoperative prescription of anti cholinesterase alone and non thymomatous MG are good prognostic factors. Thymectomy should also be considered for ocular type of MG.

\section{HYBRID APPROACH FOR TETRALOGY CORRECTION POST CLASSICAL BT SHUNT \\ Dr Vishal Agrawal, Dr Amit Mishra, Dr Shaival \\ Majumdar, Dr Pooja Vyas \\ U N Mehta Institute of Cardiology and Research}

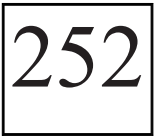

The classic Blalockâ€'Taussig shunt (CBTS) provided a viablealternative in an era when prosthetic graft materials were not available and experience with cardiopulmonary bypass in small children was limited. There were several advantages of CBTS, most importantly being the potential adaptive growth of anastomosis and predictability of blood flow, because the subclavianartery diameter prevents excessive blood flow to lungs and hence, congestivecardiac failure. Also it is very unlikely to cause pulmonary vascular disease. However a major disadvantage of CBTS is that subclavian or the innominate artery have to be sacrificed compromising the ipsilateral upper limb bloodsupply. Moreover these patients also tend to be relived from cyanosis till theygrow up due to potential growth of anastomosis, thereby relieving their symptoms and so do not present timely for complete repair. They present later in adult life when the shunt gets stenosed at some point and by this time have developed multiple collaterals that are liable to be injured during surgery for final repair. Moreover during final surgical repair the looping of CBTS for ligation needs extensive dissection with risk of recurrent laryngeal nerve injury, phrenic nerve injury, and Horner syndrome. Considering these potential complications we developed a strategy to do occlude the CBTS by transcatheterroute in a hybrid cardiac operating room. We conclude that this strategy is safe and effective and such cases highlight the importance of having a hybrid cardiac operating room.

\section{KOMMERELL DIVERTICULUM: RIGHT SIDED AORTIC ARCH WITH ABERRANT LEFT SUBCLAVIAN ARTERY:A RARE CASE}

Dr Sourabh Mittal, Dr Anil Sharma, Dr. Sunil Dixit, Dr Mohit Sharma SMS Medical College, Jaipur Rajasthan

\section{Background}

Kommerell diverticulum with a right-sided aorticarch is a rare congenital anatomical condition most often observed in adults. It can result from dilation of the anomalous origin of the subclavian artery in the following situations: left aortic arch (LAA) with anomalous origin of right subclavian artery (RSCA) (0.5-2.0\%), or right aortic arch (RAA) with anomalous origin of the left subclavian artery (LSCA) $(0.05-0.1 \%)$. Here we 
report a case of 18 year old male who was evaluated for dyspnea and choking sensation. Our diagnosis was based on seriesof test i.e. 2-D echo, barium study, aortogram, upper GI endoscopy. There waspresence of bulbous diverticulum \&amp; ligamentum arteriosum behind the oesophagus which was causing extrinsic compression over the oesophagus. Ligamentumarteriosum was doubly ligated and transected. After permittive hypotension side biting vascular clamp was applied over diverticulum and sharply cut. Interposition Dacron graft of $6 \mathrm{~mm}$ diameter was used for repositioning of subclavian artery over divided stump. Conclusion

Right sided aortic arch with aberrant left subclavian artery is a rare vascular condition which is incidental finding most of the times. Due to lack of literature in such cases treatment execution has to be individualised considering associated anomalies.

\section{LEFT ATRIAL APPENDAGE LIGATION IN CORONARY ARTERY BYPASS GRAFTING: EARLY RESULTS OF RANDOMIZED PILOT STUDY \\ Pratik Manek, Akshya Kumar Bisoi}

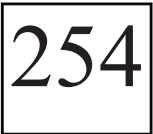

\section{AIIMS}

Objectives: Incidence of Post Op Atrial Fibrillation (POAF) is high particularly within first 7 days. The left atrialappendage (LAA) is the site of more than $90 \%$ of detected thrombi in patients with AF. Primary outcome is to assess the incidence of Stroke/Transient Ischemic Attacks post onpump coronary artery bypass grafting with or without LAA ligation. Methods: All the consecutive patients who underwent On-Pump Coronary Artery Bypass Grafting (CABG) from September 2016 to June 2017 who are at high risk of developing POAF based on the CHA2DS2-VASC score $(\& \mathrm{gt} ;=2)$ were randomized to CABG either with or without Left Atrial Appendage (LAA) ligation. Patients were followed up post operatively to rule out presence of AF or TIA or Stroke at 1 week, 1 month, 3 months, 6 months and 12 months.

Results: A total of 140 patients were randomized into two groups, Group I was with LAA ligation and Group II was without LAA ligation. None of the patients had clinically evident Stroke/ Transient Ischemic Attack during the follow up, however 8 patients in Group I and 15 patients in group II developed $\mathrm{AF}$ ( $\mathrm{p}$ value 0.1 ).

Conclusion: The technique ofepicardial suture LAA ligation during onpump CABG is a safe and cost-effective technique of LAA occlusion however, future studies and follow up is necessary to prove its significance.

\section{INFERIOR VENA CAVA LEIOMYOMA WITH INTRACARDIAC EXTENSION -OUR EXPERIENCE}

Shobhit saurav, Pankaj Gupta, Rajneesh Malhotra

Max super speciality hospital, Saket, New Delhi

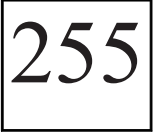

Intravenous leiomyoma with intracardiac extension is a very rare entity that necessitates surgical resection to alleviate symptoms and prevent life threatening complications. Intravenous leimyomas are benign vascular tumours of unclear cause that develops exclusively in females. These tumours can originate from the vein wall or can be a result of a vascular invasion of a uterine leiomyoma. They are usually found in the pelvic vein but can extend into the inferior vena cava and rarely into the cardiac chambers thereby producing cardiac symptoms. They form an important differential diagnosis in a female with a right atrial mass. Here we describe one such rare case which got treated successfully by surgical excision of the inferior vena cava mass extending into the right atrium and right ventricle on cardio-pulmonary bypass. The biopsy findings of the mass were consistent with an intravenous leiomyoma. She also had findings of uterine leimyomas evidenced by the abdominal ultrasound.
CYSTOTOMY WITH CAPITONNAGE IS

THE PREFERRED SURGICAL

OPTION IN THE MANAGEMENT OF

PULMONARY HYDATID DISEASE: A PILOT

STUDY FROM A TERTIARY CARE CENTRE.

Dr Nadeem Ul Nazeer

Department of CTVS, SKIMS Institute, Srinagar,

$\mathrm{J}$ and $\mathrm{K}, 190011$

\section{Background}

Pulmonaryhydatid disease is endemic to Kashmir. Various surgical options for pulmonary hydatid are enucleation, cystotomy alone, cystotomy with capitonnage, lobectomy and pneumonectomy.

Methods

The study included analysis of 610 patients of pulmonary hydatid disease treated surgically at our tertiary care institute.

Results

The most common presenting symptoms were cough and hemoptysis. Bilateral lung involvement was seen in 11 percent patients. Concomitant liver cysts were seen in 8 percent patients. Cystotomy with capitonnage was the mainstay of treatment in most of the cases. Albendazole was started from first post operative day. In hospital mortality was less than one percent. Six percent patients developed complications and few of them needed reoperation.

Conclusion

Cystotomywith capitonnage is the preferred surgical procedure for the treatment of pulmonary hydatid disease. This procedure reduces hospital stay and has lesser complications.

\section{UNFOLDING THE OLD PAGES OF CARDIOPULMONARY BYPASS: HISTORICAL OVERVIEW}

Dr. Manpal Loona, Dr. Vaibhav chugh ;

Dr. Narender S.Jhajharia; Dr. V. Grover; Dr. V. K. Gupta

ABVIMS \& Dr. Ram Manohar Lohia Hospital, New Delhi

Cardiac surgery without cardiopulmonary bypass was just an imagination in 19 th century.

Cardiac surgery was confined to emergency cases in early 20thcentury. Thereafter, cardiac surgery dimensions spread to surgeries that couldbe performed without cardiopulmonary bypass like The Blalock Taussig shunt, Patent ductus, Coarctation of Aorta, Mitral Commisurotomy. In early 1950s, Gross well, performed atrial septal defect closure using hypothermia. Further, in various attempts to discovery to bypass the heart, it was concluded one needs a method of preventing the blood from clotting, to pump the blood, a method to ventilate the blood and a basic understanding of physiology of the circulation. With its journey to development, several obstacles were encountered till mid of 1950s; Dr. John H. Gibbon performed first cardiac surgery using cardiopulmonary bypass. Thereafter, several surgeons like C. Walton Lillehei, Dr. J. W. Kirklinetc. contributed to cardiac pulmonary bypass to facilitate better outcomes. This historical overview emphasizes towards the new cardiac surgeons, how he journey of cardiopulmonary bypass happened and problems faced to reach new era of cardiac surgery.

\section{ADULT CARDIAC SURGERY: COMPARISON BETWEEN DEL NIDO VS. ST. THOMAS \\ Dr. Manpal Loona, Dr. Dhananjay K. Bansal,}

Dr. Rahul Bhushan, Dr. VaibhavChugh, Dr. V. Grover ABVIMS \& Dr. Ram Manhar Lohia Hospital, New Delhi

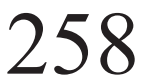

Introduction: Del Nido (DN) cardioplegia being widely used in paediatric cardiac surgery is gaining its usage in adult cardiac surgery over time. 
Aim: Compare safety of Del Nido over St. Thomas Cardioplegia solutions in adult cardiac surgery.

Methods: Of the 120 adult patients who underwent MVR/DVR/AVR were analysed retrospectively, who received Delnido cardioplegia $(n=60)$ and St. Thomas Cardioplegia $(n=60)$. We retrospectively analysed the collected data of all consecutive on pump patients to assess Cross clamp time (X clamp time), cardiopulmonary bypass time (CPB time, post cross clamp defibrillation rates.

Results: The aortic cross clamp and bypass times were shorter with DN than ST $(88.76 \pm 32.26$ vs. $104.56 \pm 33.33$ and $122.59 \pm 31.44$ vs. 141.76 \pm 32.24 , p value $\& ; 0.05$ respectively). DN group showed significant reduction in Post X clamp defibrillation rate than ST group (4 vs. 12, p value \&;0.05).

Conclusion: Delnido reduces $\mathrm{X}$ clamp and CPB time and has less post $\mathrm{X}$ clamp removal defibrillation rate in comparison to delnido in adult cardiac surgery.

\section{PRIMARY INTRATHYMIC LYMPHANGIOMA: A RARE CASE REPORT \\ Dr. Utkarsh Sanjay Sanghavi, Dr. Hiremath CS, Dr. Shekhar Rao \\ Sri Sathya Sai Institute of Higher Medical Sciences, Bengaluru}

Introduction: Lymphangiomas are relatively uncommon lesions of thelymphatic channels, considered as true neoplasms by some and as malformations by others, mostly occurring during the first 2 years of life. The most common site is the head/neck region. Mediastinal lymphangioma is very rare, accounting for $0.7-4.5 \%$ of all mediastinal tumors with only 20 cases reported in literature.

Case report: A 16-monthold male child presented with complaints of shortness of breath restricting his daily activities. Air entry on left side of the chest was reduced. Chest X-ray showed opacity in left hemithorax with a mediastinal shift to the right. CT thorax reveled a large cystic lesion measuring $86 \times 71 \times 53 \mathrm{~mm}$ in the left hemithorax closely abutting the thymus gland and left ventricle with two septations within the lesion causing a mass effect and mediastinal shift to the right. Median sternotomy was done. Three large sacs were noted. During sternotomy one sac had ruptured the remaining mass measured $95 \times 50 \times 70 \mathrm{~mm}$ and weighed approximately $32.9 \mathrm{gms}$ and was strongly adhered to the surrounding structures including the phrenic nerve and the diaphragm. The cyst was dissected completely and away from the surrounding structures. The HPE revealed thymic follicle with flattenedepithelium, eosinophilic material within the cystic lumen, lymphoid aggregates with germinal centre formation in the wall suggestive of lymphangioma. To our knowledge, our case represents the second description of true thymic lymphangioma with an unequivocal intrathymiclocalization. Conclusions: Thymic lymphangioma should always be taken into consideration in the differential diagnosis of mediastinal cystic masses in all patients.

\section{CARDIOPULMONARY BYPASS FOR RESECTION OF RENAL AND INFERIOR VENA CAVA} TUMOURS: EXPERIENCES FROM OUR SERIES

Vipin Balachandran, Linna Huang, Casey Garnham, Lara Beukes, Taranpreet Singh, Alexander Grant, Alan Boyd John Hunter Hospital

\section{Background:}

Renal tumours often grow into the inferior vena cava (IVC) through the renal vein in about $5-10 \%$ of all cases necessitating extensive tumour thrombus removal and IVC reconstruction. We are presenting our experiences with 13 casesnecessitating cardiopulmonary bypass (CPB) with and without circulatory arrest.

Aims/Objectives:

1. To evaluate the technical issues of cardiopulmonary bypass for management of tumour thrombus in the IVC 2 . To evaluate the safety of circulatory arrest in the management of IVC tumour thrombus and subsequent reconstruction of the IVC

Methods:

1. All patients were evaluated at a multi-disciplinary meeting involving cardiothoracic, urological and general surgeons in addition to oncologists, pathologists, radiologists and perfusionists. 2. 13 patients were selected for management of tumour thrombus in the IVC with cardiopulmonary bypass support. 3. A midline laparotomy was the initial incision for all patients. All patients had central bypass via a edian sternotomy 4. Cooling to various temperatures were carried out depending on the extent of the tumour thrombus and the complexity of the reconstruction. 5. Separation from $\mathrm{CPB}$ was done after active warming to 37 degrees $\mathrm{C}$.

Results:

The mean CPB times was $102.62 \pm 41.26$ minutes and mean circulatory arrest times (when used) was $23.92 \pm 12.04$ minutes. In the immediate post-operative period, all patients required noradrenaline (100\%), 3 patients $(23 \%)$ required adrenaline and 4 patients $(29 \%)$ required dobutamine for haemodynamic support. All patients $(n=13 ; 100 \%)$ survived 30 days post operatively and were discharged home. The average hospital stay was $16.85 \pm 13.78$ days. The all cause mortality within the first 5years was $38 \%(n=5)$.

Conclusion:

1. Central cardiopulmonary bypass with or without hypothermic arrest is a safe and reliable tool in the armamentarium of the surgeon operating on an IVC tumour thrombus. 2 . The morbidity and mortality associated with this rather extensive procedure is acceptable with a 5 year survival rate over $62 \%$. 3. A team approach is imperative in the management of an IVC tumour thrombus and is directly related to better patient outcomes.

\section{MEDIASTINAL MASS WITH MUCOCUTANEOUS BLISTERS}

Dr. Sampaul Richard, Dr. Sreekar, Dr. Suresh

St. Johns Medical College and Hospital

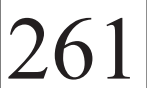

AIM : To present an interesting case of Mediastinal mass and Paraneoplastic pemphigus in a 52 year old patient and its management .MATERIAL AND METHOD : Herein we report a case of 52 years old patient who came to the Department of Dermatology with complaints of skin lesions on the body along with cough and dyspnea. Routine Chest $\mathrm{X}$ ray showed widening of the mediastinum and on further imaging, an anterior mediastinal mass was present. Evaluation, diagnosis, differential diagnosis and its management of this rare case will be discussed .RESULT : The tumor was surgically excised and sent for histopathological examination, which revealed a Follicular dendritic cell sarcoma Different treatment regimen was used to treat the skin lesions which on skin biopsy showed pemphigus vulgaris. But there was remission of the lesions in our patient post surgery and chemotherapy. CONCLUSION :The existence of primary neoplasm of the follicular dendritic cell was first described in 1986, since that time scattered cases have been reported in the literature. However, to our knowledge so far only a handful of combined Mediastinal mass and pemphigus vulgaris cases have been reported in the literature which needs to be monitored for the benefit of the patients

\section{BOERHAAVES SYNDROME: A THORACIC

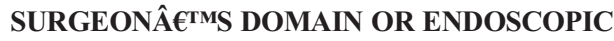 STENTING OR COMBINED MODALITY TREATMENT}

Bhushan Dinkar Thombare

Medanta - The Medicity

Objective:

Spontaneous esophageal rupture was previously known as Boerhaavescribedby Dr. Herman Boerhaave in 1724. The successful 
treatment of Boerhaaves syndrome is in itself a challenge, especially asesreferred late due to delayed diagnosis or adoption of minimal invasive option of esophageal stenting. Delayed diagnosis results in dangerous consequences such as serious mediastinal infection and sepsis, which are accompanied by high mortality. Moreover, the treatment of Boerhaaves syndrome is related to many factors, and there is as of yet no definitive treatment. The aim of this case series is to present these rare cases, and to discuss the clinical presentation and management options of these rare cases.

Materials and Methods

A retrospective analysis of 5 unique patients with Boerhaaves syndrome managed at tertiary centre, considering the following parameters: etiology, symptoms, time interval between onset and treatment, therapeutic methods, position and size of rupture, complications, outcome, and length of stay was done.

Result

5 patients of Boerhaaves syndrome, Male: Female :: 2:3, average age was53 years. 2 patient underwent esophageal stenting one early and other delayed, 2 patients underwent surgical repair one early repair and other one delayed repair, and one patient the perforation healed by conservative means. Feeding Jejunostomy was done inpatients which underwent surgical repair. 4 patients underwent decortications and drainage of empyema, 2 of them were bilateral and 2 of them were unilateral one left side other right side, food particles were retrieved from one of the patients chest after 8 months who had refused surgery at first instance after stenting. Empyema and development of sepsis were the common complication. One patient expired due to sepsis leading to multi organ failure. Conclusion

Esophageal stenting, early or late Surgical repair, Stenting along with surgical treatment of complications remain the valid options in treatment of Boerhaaves syndrome according to time interval in treatment. The choice of treatment depends upon critical and continuous assessment of the patients clinical status, the extent and duration of the leak, and the presence of sepsis. Although Minimally or less invasive treatment options like esophageal stenting have been applied successfully, timely referral to thoracic surgeon for management of complications should be considered early, apart from multi-disciplinary treatment.

\section{COMBINED ANTERIOR WITH POSTERIOR ROOT ENLARGEMENT TO PREVENT PPM IN HYPOPLASTIC AORTIC ANNULUS.}

Dr. Dinesh kumar. R, Dr. Hiremath. C.S, Dr. Shekhar Rao

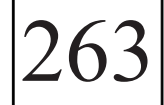

Sri Sathya Sai Institute of Higher Medical Sciences

\section{Introduction:}

Aortic valve replacement in the young or with a hypoplastic aortic root remains a technical challenge, and in such cases enlargement of the aortic annulus is required to prevent PPM. Various techniques to achieve this enlargement have been described. The annular position for an aortic valve prosthesis is appealing and is the location of choice if a prosthesis of adequate size can be accommodated. We present a patient with severe AS with severely hypoplastic root.

Case Report: Forty three years female presented with angina and easy fatigability NYHA functional class 2 and syncope since 10 years. She had undergone balloon dilatation of aortic valve in 2008 for severe AS. Upon evaluation, she was found to have Bicuspidaortic valve with severe AS with aortic annulus of $14 \mathrm{~mm}$. Intraoperatively, annulus calcified with calcium extending into interventricular septum. Nunez posteriorroot enlargement with Konno-Rastan anterior root enlargement was done and annulus increased to $23 \mathrm{~mm}$. \#23 TTK Chitra mechanical tilting disc valve was implanted. Cross clamp time was 352 minutes and CPB time was 462 minutes. TEEshowed valve prosthesis well seated, gradient- $16 / 7 \mathrm{mmHg}$, mild biventricular dysfunction. Immediate post-operative she had complete heart block which reverted to sinus on POD 2. She was discharged on POD 7. Echo atdischarge showed aortic prosthesis well seated, gradient-18/9 mmHg, normal biventricular function. At 3 months follow-up, she is doing well and echo showed prosthetic valve well seated, gradient$10 / 5 \mathrm{mmHg}$, normal biventricular function.

Conclusion:

Hypoplasticaortic root with severe AS needs to be addressed appropriately. Although aortic root enlargement carries significant morbidity it improves the quality of life in patients who need it. We conclude that AVR with combined anterior and posterior root enlargement can be done safely for severe AS with hypoplastic aortic root and is essential to prevent PPM in selected patients.

\section{A RARE CAUSE OF CARDIAC TAMPONADE IN A PREVIOUSLY YOUNG HEALTHY WOMAN.}

Dr. Steve Babu, Dr. Sreekar Balasundaram, Dr Suresh Kumar St. Johns Medical College Hospital.

1)AIM/OBJECTIVE: To present a rare cause of cardiac tamponade in a previously young healthy woman.2) MATERIAL AND METHOD: Patient 22 yr old female presented with fever and clinical signs of pericardial tamponade, initially managed with pericardial catheterisation and medical therapy but taken up for median sternotomy, pericardiectomy and bilateral decortication owing to the failure of medical management and worsening of symptoms.3)RESULT: Diagnosis of cardiac tamponade secondary to Actinomycetespyopericardium with mediastinitis and bilateral loculated pleural effusion was made. Patient post therapy survived and has no recurrence in the last 1 year.4) CONCLUSION:. It is important to recognize that in such conditions, appropriate medical and timely surgical therapy can decrease morbidity and mortality.

\section{A RETROSPECTIVE ANALYSIS OF THE EFFECTS OF DEL NIDO CARDIOPLEGIA ACROSS A RANGE OF CARDIAC CASES}

Vipin Balachandran, Rebecca Tee, Lachlan Gan, Priyanka Paul, Peng Seah, Ross Mejia

John Hunter Hospital

Background: del Nido (DN) cardioplegia was developed as a myocardial protection strategy for immature hearts in the paediatric population. However, there has been a rapid uptake into adult cardiac surgery due to its single dosing strategy, potential superior myocardial protection and lower cardiopulmonary and cross clamp times. In spite of a plethora of observational studies, the majority of studies have looked at very specific populations and none have included emergent cases. We are pleased to present the findings from the John Hunter Hospital in over 300 patients across a wide case range including emergent and salvage cases. Aims:-To evaluate the haemodynamic parameters resulting from $\mathrm{DN}$ cardioplegia across a range of cardiac cases including emergency and salvage cases-To evaluate inotrope usage post cardiotomy after the use of DN across a range of cardiac cases including emergency and salvage cases-To evaluate total bypass (CPB) and cross clamp times (AXC) with the use of DN across a range of cardiac cases including emergency and salvage casesMethodology:309 cases requiring cardiopulmonary bypass and where DN cardioplegia was used were analysed between 2015 and 2018 by reviewing patient records. All cases including emergent and salvage cases were included in this analysis. Patients were excluded if perfusion records did not identify total cardiopulmonary bypass and aortic cross clamp times. The final analysis was done on 307 cases. Results: The mean CPB time was 106.21 +/- 2.60 minutes and the mean AXC was $71.19+/-2.02$ minutes. The majority of isolated cases required only 1 dose (1L) of DN whereas cases exceeding 90 minutes had 2 doses of half dose $(500 \mathrm{~mL}) \mathrm{DN}$. Cardiac indices were between $2.35+/-0.6 \mathrm{~L} / \mathrm{min} / \mathrm{m} . \mathrm{m}$ 
and $3.45+/-0.82 \mathrm{~L} / \mathrm{min} / \mathrm{m} . \mathrm{m}$. An evaluation of individual cohorts of surgeries is beyond the scope of this abstract and will be presented at the meeting. Conclusions: We have evaluated DN in a 'real world' scenario and found it to be a safe alternative to standard hyperkaelemic solutions for myocardial protection across a wide range of cardiac cases irrespective of urgency. Its single dose strategy decreases interruptions to the operation and may provide superior myocardial protection and decreased cardiopulmonary bypass times.

\section{GIANT CORONARY ARTERY ANEURYSM WITH MODERATE AORTIC REGURGITATION Dr. A. Mohammed Idhrees, Dr. K. Mukesh, Dr. Ketak Nagare, Dr. Mohamed Ibrahim, Dr. Aju Jacob, Dr. Bashi V. Velayudhan SRM Institutes for Medical Science}

Introduction Coronary artery aneurysms (CAAs) are uncommon and describe a localized dilatation of a coronary artery segment more than 1.5-fold compared with adjacent normal segments. Dilatations that exceed $4 \hat{a} € \% o c m$ in diameter as giant coronary aneurysms. Giant coronary artery aneurysms are rare, with a reported prevalence of $0.02 \%$ to $0.2 \%$. Here we present a case with giant coronary artery aneurysm (GCAA) associated with moderated aortic regurgitation (AR). This association of GCAA and AR has not been reported previously and is the first in the world to the best of our knowledge. Case History65 year old male presented with history of dyspnoea on exertion. His Echocardiogram showed trileaflet aortic valve with moderate aortic regurgitation. His left ventricular function was good. CT aortogram revealed a large right coronary artery aneurysm of $6 \times 4 \mathrm{~cm}$ and a left main coronary artery aneurysm of $2 \times 2 \mathrm{~cm}$. He underwent Bentall's procedurewith coronary artery bypass grafting. He had an uneventful postoperative period and is on follow up for last 2 years. Conclusion Giant coronary aneurysms are very rare, and simultaneous giant coronary artery aneurysm with aortic regurgitation has not been reported previously. Surgical treatment should be considered in order to prevent potential complications.

\section{COMPARISON OF IMMEDIATE POST OPERATIVE OUTCOME OF CONVENTIONAL CARDIOPULMONARY BYPASS AND CARDIOPULMONARY BYPASS WITH MODIFIED PLASMA ULTRAFILTRATION : A PROSPECTIVE OBSERVATIONAL STUDY.}

Manoop B, Abdul Rasheed M H

Government Medical College, Thiruvanathapuram.

Aim - To determine whether simplified modified ultrafiltration ( S-MUF) with better hemodynamic advantage, improves immediate post operative Blood gas analysis and hematocrit values, decreases post operative blood loss and fall in central venous pressure values. Comparison of total amount of inotropic supports needed in immediate post operative period, blood support - FFP, platelets and packed cells. Methodology - Sample size of $n=13$, however sample size was increased to 30 pts to improve significance of study. Convenient sampling, consecutive cases meeting eligibility were included. Results - MUF was higher risk group $\mathrm{p}=0.02$. Hemoconcentration was successfully achieved in MUF group with lower blood loss and transfusion requirement. Mortality in both groups were comparable. Conclusion - MUF is a safe procedure which successfully achieves hemoconcentration, lowers blood loss and transfusion requirement after cardiac surgery.
OUTCOME OF LESS INVASIVE LEFT

VENTRICULAR ASSIST DEVICE IMPLANTATION THROUGH LEFT

\section{THORACOTOMY WITH MINI-STERNOTOMY}

268

Dr Sanjay Kumar, Dr Vladimir Shumaster

1 Department of Cardiothoracic Surgery, Institute of Medical Sciences, Banaras Hindu University, Varanasi, India; 2 Division of Cardiac Surgery, Yale New Haven Medical School, New Haven, Connecticut, USA

\section{OBJECTIVES:}

The morbidity and mortality associated with Right ventricular (RV) failure after left ventricular assist device (LVAD) implantation continues to be a major complication. In this study, we studied the hypothesis that a less invasive approach to implantation would preserve RV function and mortality relative to a conventional full sternotomy (CFS) approach. METHODS:

We reviewed all consecutive patients who underwent LVAD implantation between January 2012 and December 2018. The primary outcome studied was severe RV failure and associated morbidity and mortality. Patients were divided into two groups as per surgical approach: less invasive left thoracotomy with mini-sternotomy (LTMS) and CFS

RESULTS:

We studied 76 patients (LTMS: 31, CFS: 45) were identified. The median INTERMACS Interagency Registry for Mechanically Assisted Circulatory Support) score was significantly worse in the LTMS compared to the CFS cohort, and there was a trend towards higher RV failure scores and Heart Mate II mortality scores. Preoperative RV dysfunction, in pulmonary artery pulsatility index and RV stroke work index were similar between the 2 groups. The cardiopulmonary bypass time was significantly shorter in the LTHS group (61 vs95 min, $\mathrm{P}=.001$ ). The operative time did not significantly differ between the two groups. Improvement in RV function, along with a change in pulmonary artery pulsatility index, was significantly greater in the LTHS cohort. The incidence of postoperative severe RVfailure was significantly reduced in the LTHS group ( $16 \%$ vs $39 \%, \mathrm{P}=0.030)$,along with the need for temporary right ventricular assist device ( $5 \%$ vs $29 \%, \mathrm{P}=0.005$ ). There was a trend towards improved survival Kaplan-Meier 1-year survival in the LTHS cohort ( $89 \%$ vs $52 \%, \mathrm{P}=0.056$ ).

CONCLUSIONS:

In this cohort, minimal access LVAD implantationappears to be associated with reduced postoperative RV-failure, and improved survival compared to conventional LVAD implantation.

\section{LATE PRESENTATION OF ANTERIOR MEDIASTINAL ABSCESS, PRESENTING AS A CHEST WALL ABSCESS AFTER BIOPSY OF CA VALLECULA}

Dr. Yousuf Mohammed Rafi, Dr. Shivkumar swamy, Dr. Satyaprakash, Dr. Sosle, Dr. Ashit Shetty

Sri Jayadeva Institute of Cardiovascular Sciences and Research

Introduction: Anterior mediastinal abscesses are uncommon without preceding trauma or instrumentation. Non-traumatic mediastinalabscesses can occur from direct extension along fascial planes from odontic, orretrophayngeal infections, following osteomyelitis of ribs or vertebrae or by hematogenous or lymphangitic spread from distant sites.

Case Presentation: A 58 year old man presented with a large $15 \times 7 \mathrm{cms}$ swelling over upper chest on either side of sternum, which was tender. Patient denied fever with chills. There was a history of a Biopsy for a vallecular mass 20 days prior to current presentation. Total counts were elevated. Chest X-ray PA, Lateral showed a soft tissue swelling over anterior chest wall with extension into anteriomediastinum. CT-Thorax was done which demonstrated an Anterior mediastinal Abscess draining into upper chest suprasternally. CT Neck was done additionally which demonstrated hypodense collections within left parapharyngeal, 
paralarygenal spaces, left pyriform fossa, left strap muscles. Anterior Chest wall collection was drained, and pus fromanterior mediastinal area was evacuated. Fluid from the abscess grew Klebsiellapneumoniae. Biopsy of the Vallecular mass revealed squamous cell Carcinoma. Discussion: Anterior mediastinal abscess can occur from direct extension along fascial planes from retrophayngeal infections, as is postulated in this case. It is a rare, but potential complication following odontic, endoscopic instrumentation or infection. Kliebsiella pneumoniae is commonly isolated. The incidence of infective complications is low, but can rapidly escalate into life threating condition. High level of suspicion is required for diagnosis

\section{A NEW TECHNIQUE OF \\ HETEROTOPIC HEART TRANSPLANTATION WITH DIRECT PULMONARY ARTERY ANASTOMOSIS}

Dr Prashant Vaijyanath, Dr Charumathi dasarathan

Kovai Medical Center and Hospital

Introduction The current technique for heterotopic cardiac transplantation in patients with high pulmonary vascular resistance entails the interposition of a Dacron conduit lbetween the native and donor pulmonary artery (PA) as itis not possible for the donor PA to reach the recipient PA (Figure 1). The original technique of Bernard and Losman2was abandoned due to intractable arrhythmias. Da Silva etal. 3proposed an anastomosis between the donor main PA andthe inferior margin of the recipient right $\mathrm{PA}$. We describe a technique of a direct PA anastomosis between the anterior surface of the recipient right PA andthe donor left PA an in end-to-side fashion after closing the donor right PA with suture. We believe that this technique confers an excellent anatomic orientation, thereby reducing the chances of the kinking and torsion of the anastomosis. Surgical Technique We used this technique in a 48 -year-old male patient of end-stage ischemic cardiomyopathy with prior multiple percutaneous coronary interventions and cardiac resynchronization therapies. The calculated pulmonary vascular resistance was $7.1 \mathrm{WU}$ and the systolic PA pressure was $82 \mathrm{mmHg}$ with a systemic pressure of 95/50.The patientâ $€^{\mathrm{TM}_{\mathrm{S}}}$ severe pulmonary hypertension precluded ortho topic transplantation. Donor Heart Preparation After standard donor cardiectomy, the ostium of the right pulmonary veins and the right PA was closed. The inferior vena cava (IVC) was not closed, and nor was a posterior opening made in the superior vena cava (SVC)-right atrium junction of the donor heart for a cavo-atrialanastomosis. Recipient Operation After a standard cardiopulmonary bypass, right-sided pleuro-pericardial reflection was divided to create space for the donor heart in the right pleural cavity. The recipient SVC was transected near the right atrium, its lower stump was sutured, and its upper stump was laterally mobilized, which exposed the right PA. A left atrial incision was made medially to the right superior pulmonary vein and extended in the interatrial groove. The donor heart was placed in the right side of the chest, and the 2 left atria were sutured in a position that allowed the donor left PA to easily reach the recipient right $\mathrm{PA}$. The anterior surface of the recipient rightPA was opened and a cobra hood posterior slit was made on the donor left PA, almost extending to the main PA, and a direct anastomosis was achieved without tension. This was followed by an end-to-end donor/recipient SVC anastomosisand then an end-to-side anastomosis of the donor IVC to the recipient IVC as low as possible, allowing the lower body venous return of the recipient to reach the donor right atrium. The donor ascending aorta, which is usually long, wasanastomosed as distally as possible in the recipient ascendingaorta to create space and access to the PA anastomosis incase of bleeding (Figure 2). The patient was easily weaned off cardiopulmonary bypass with good hemodynamic and functional recovery. The patientâ $€^{\mathrm{TM}_{\mathrm{S}}}$ systolic PA pressure had fallen to half systemic arterial pressure by the time he left the intensive care unit. Discussion We believe that the method herein expounded has certain merits. The technique can prevent the theoretic complications of a PA prosthetic graft such as compression, infection, thrombosis, fibrosis, and difficulty in sternal closure. Moreover, achieving a bicaval anastomosis seems to have dual advantages. The first advantage on the SVC side is that the closure of the recipient cardiac end unloads the dysfunctional native heart and endomyocardial biopsy is facilitated because the SVC anastomosis leads the bioptomeforceps easily to the donor right ventricle. The other positive point on the IVC side is the sharing of lower body blood and burden between the recipient and donor heart. References1. Novitzky D, Cooper DK, Barnard CN. The surgical technique ofheterotopic heart transplantation. Ann Thorac Surg 1983;36:476-482.2. Barnard CN, Losman JG. Left ventricular bypass. S Afr Med J1975;49:303-312.3. Da Silva JP, Cascudo MM, Baumgratz JF, Vila JH, Wafae Filho M,de Carvalho Neto DO, Da Fonseca L. Heterotopic heart transplan-tation: a direct pulmonary artery anastomosis technique. J Thorac Cardiovasc Surg 1994;108:795

\section{ROLE OF ULTRASOUND GUIDED FOAM SCLEROTHERAPY IN VARICOSE VEINS: MYTH OR NECESSITY}

Dr. Ansul Kumar

Department of CTVS, Rajendra Institute of Medical Sciences,

Ranchi/Jharkhand

\section{Background:}

Varicose veins affect up to $25 \%$ of women and $15 \%$ of men in the western world and incidence is apparently low in India. Male preponderance was observed with male to female ratio 14:1 in Indian scenario. Sclerotherapy involves the injection of a sclerosing agent directly into the superficial veins. The most commonly used is sodium tetradecyl sulphate. The direct contact with detergent causes cellular death and initiates an inflammatory response, aiming to result in thrombosis, fibrosis and obliteration (sclerosis). Blood deactivates the action of the sclerosing agent and the doses administered need to be limited toavoid adverse effects, causing a tradeoff between poor efficacy and safety.

Aim:

In this study outcomes of Non-USG guided injection sclerotherapy were studied and analysed among patients coming to RIMS, Ranchi which is a tertiary care centre in Jharkhand.

Methods:

A total of 50 cases who underwent Non-USG guided foam sclerotherapy were studied out of which, all had obliteration of Varicose Veins. The secondary outcomes were symptomatic improvement, ulcer healing, recurrence, and adverse events. Adverse effects were pain (86\%), pruritis (32\%), swelling (14\%), thrombophlebitis (16\%) and skin ulceration $(2 \%)$. Complete obliteration was achieved after one injection in all cases. Thrombosis and neurologic events were not seen.

Conclusion:

Foam Sclerotherapy, even Non-USG guided, appears to be a safe and effective outpatient therapy for the treatment of Varicose Veins and hence can be used even in set-ups lacking the facility of USG thus saving patient cost and resources. 